\title{
BROOKHAVEN NATIONAL LABORATORY
}

\section{Annual Report \\ July I, 1953}
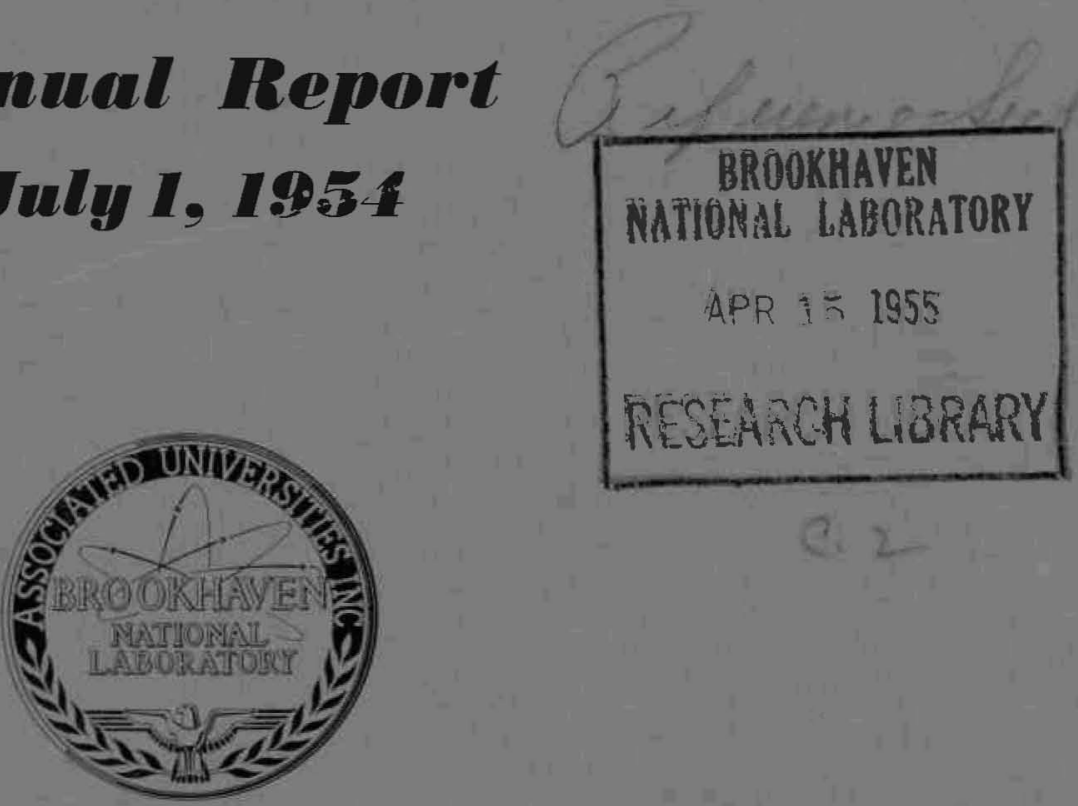

\section{Associated Universities, Inc.}

under contract with the

\section{United States Atomic Energy Commission}




\section{Annual Report July 1, 1954}

\section{BROOKHAVEN NATIONAL LABORATORY \\ Associated Universities, Inc. \\ Upton, New York}


Brookhaven National Laboratory is operated under a contract between the United States Atomic Energy Commission and Associated Universities, Inc. This, the fifth in a series of unclassified Annual Reports, gives an account of the progress of the Laboratory during the period July 1, 1953 - June 30, 1954 , and its plans for the future. It is submitted under the terms of Contract No. AT-30-2-GEN-16 between Associated Universities, Inc., and the Atomic Energy Commission.

Previous reports in this series are:

BNL 74 (AS-4) July 1, 1950

BNL 131 (AS-5) July 1, 1951

BNL 196 (AS-6) July 1, 1952

BNL 246 (AS-7) July 1, 1953

Printed at Upton, New York, for distribution to persons and organizations associated with the national atomic energy program.

March 1955

1400 copies 


\section{CONTENTS}

INTRODUCTION $\ldots \ldots \ldots \ldots \ldots \ldots \ldots \ldots \ldots \ldots \ldots \ldots \ldots \ldots \ldots \ldots$

PHYSICAL SCIENCES AND ENGINEERING

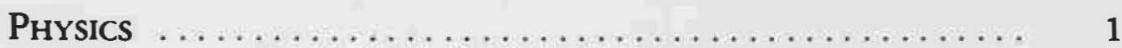

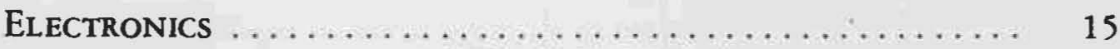

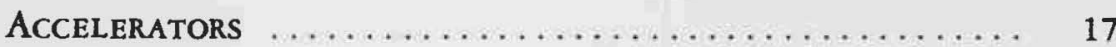

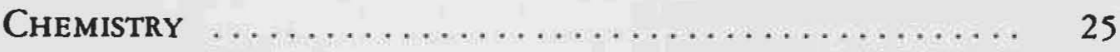

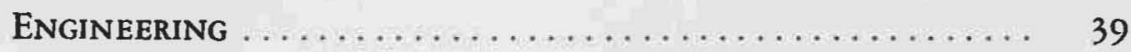

LIFE SCIENCES

BIOLOGY .................... 47

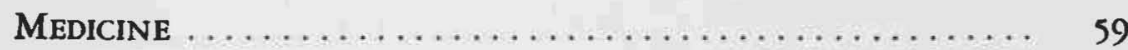

TECHNICAL OPERATIONS AND SERVICES $\ldots \ldots \ldots \ldots .69$

ADMINISTRATION AND OPERATIONS $\ldots \ldots \ldots \ldots \ldots \ldots \quad 83$

APPENDIX - A. Unclassified Publications, July 1,1953 - June 30, $1954 \ldots \ldots \ldots \ldots \ldots 95$

B. Officers and Scientific Staff $\ldots \ldots \ldots \ldots, 110$ 


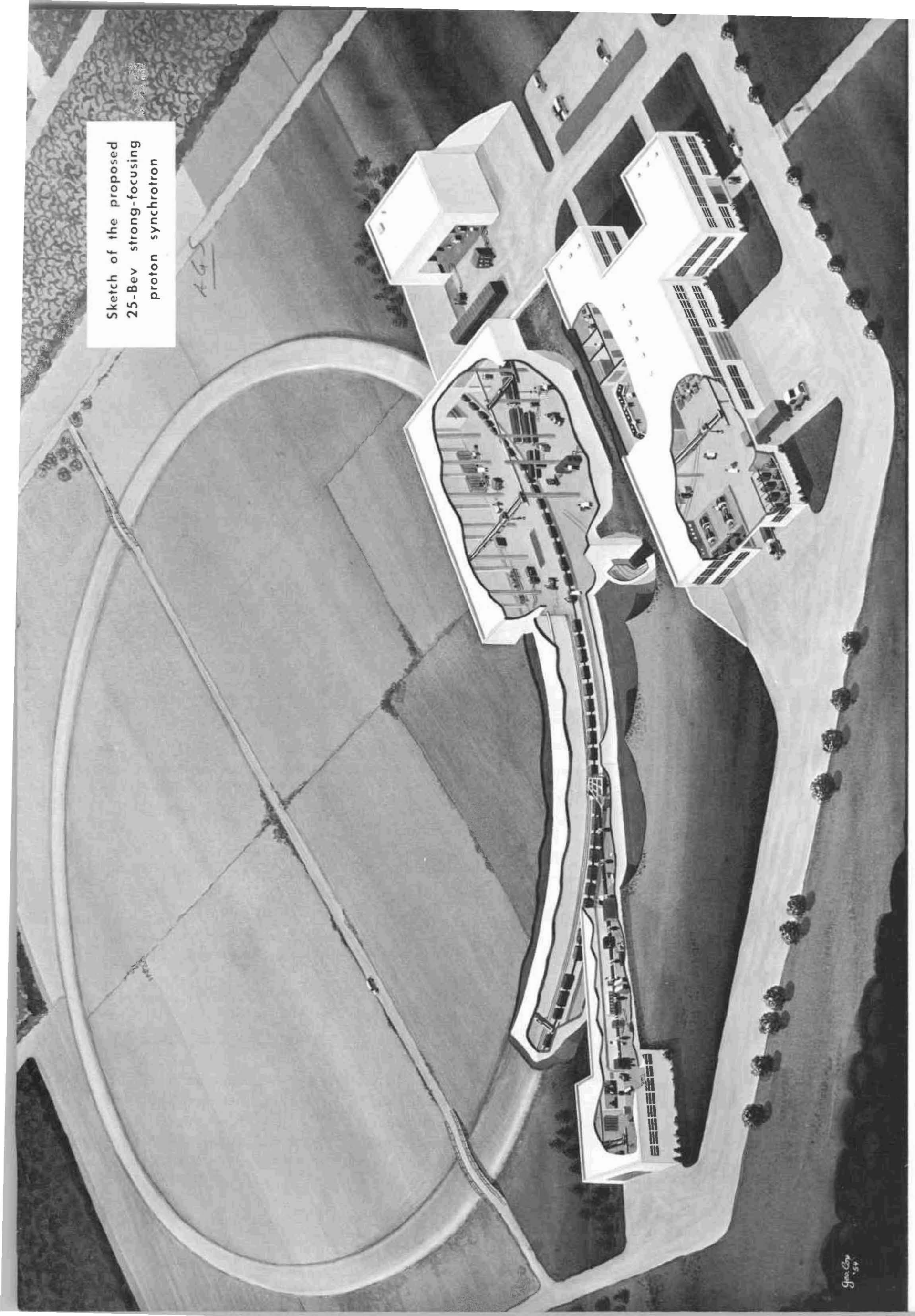




\section{N T R O D U C T I O N}

This annual report of Brookhaven National Laboratory describes its program and activities for the fiscal year 1954. The progress and trends of the research program are presented along with a description of the operational, service and administrative activities of the Laboratory. The scientific and technical details of the various research and development activities are covered more fully in scientific and technical periodicals and in the quarterly scientific progress reports and other special reports of the Laboratory. (For the Laboratory's output in publications since 1946, see Figure 1.) Hence the activities of the Laboratory service organizations are treated more fully in this report whereas research activities are covered in summary.

Brookhaven National Laboratory is operated by Associated Universities, Inc. under contract with the United States Atomic Energy Commission. Nine northeastern universities sponsor Associated Universities, Inc. Two representatives from each university, one an administrative officer of the university and other a scientist, join together to form the Board of Trustees of AUI. AUI has appointed five visiting committees to Brookhaven National Laboratory to provide a continuing independent evaluation of the research program of the Laboratory. These visiting committees cover the fields of physics, chemistry, biology, medicine and engineering and report annually to the Board of Trustees. Their reports, which are on file with the Corporation, are made available to the Director of the Laboratory for his guidance and assistance.

\section{PRESENT RESEARCH FACILITIES}

During the report year, the Cosmotron and the nuclear reactor supported a large fraction of the research effort of the Laboratory. Each machine turned in an exceptionally fine performance record.

At the Cosmotron there is always a greater demand for machine time by the experimentalists than is actually available. Improvements are continually being made on the machine in order to effect a greater efficiency in the research program. Through the activation of the pole-face windings, the beam energy was brought to its full design value of $3 \mathrm{Bev}$ and improvements in operation throughout the year have provided for an increase in beam intensity of about a factor of 10. Experience has been gained in simultaneous operation of several experimental equipments through use of switching devices and beam selection.

The volume of research carried out with the nuclear reactor during the past year has continued at peak load. The number of research projects is essentially at capacity with about 66 separate experimental arrays in use at the three faces and on top of the reactor. Use of the reactor by non-BNL personnel has increased during the year, primarily among industrial organizations, some of which are carrying out work under AEC contracts. The reactor operated about $83 \%$ of the time throughout the year for a total of 7,244 megawatt days, as contrasted with 7,500 magawatt days accumulated during the preceding year. This reduction was primarily due to one long shutdown period for a special experiment involving the reactor itself and an inforced lower level of operation during most of the winter in order to meet a severe budget restriction existing at that time. The normal level of operation of the reactor is set as a compromise between research demand for high flux and cost to operate. The Brookhaven reactor is an air-cooled reactor which requires substantial fan power. Full operation at maximum power level which would require all five fans could be carried out at an annual power cost to the Laboratory of $\$ 500,000$ per year. Present normal operation with only four fans costs about $\$ 420,000$ for electrical power alone.

Modifications and repairs of various magnitudes have been made on the 60 -inch and the 18-inch cyclotrons and on the research electrostatic accelerator. Performance of the 60-inch cyclotron has been improved. In the winter of 1954 , a new set of dees was installed which thereby eliminated many of the previous troubles and since the change, the cyclotron has operated satisfactorily for target preparation and 
BOARD OF TRUSTEES

of

ASSOCIATED UNIVERSITIES, INC.

As of June 30, 1954

COLUMBIA UNIVERSITY

Frank D. Fackenthal (Honorary)

George B. Pegram

I. I. Rabi

CORNELL UNIVERSITY

Theodore P. Wright

Franklin A. Long

HARVARD UNIVERSITY

Edward Reynolds

Norman F. Ramsey

JOHNS HOPKINS UNIVERSITY

P. Stewart Macaulay

William D. McElroy*

MASSACHUSETTS INSTITUTE OF TECHNOLOGY

Edward L. Cochrane

Jerrold R. Zacharias

PRINCETON UNIVERSITY

George A. Brakeley

Joseph C. Elgin

UNIVERSITY OF PENNSYLVANIA

William H. DuBarry

Francis C. Wood

UNIVERSITY OF ROCHESTER.

LaRoy B. Thompson

William S. McCann

YALE UNIVERSITY

C. Hamilton Sanford, Jr.

William W. Watson

*Elected as successor to H. Keffer Hartline who resigned on June 18, 1954 


\title{
COMMITTEES TO VISIT THE DEPARTMENTS OF \\ BROOKHAVEN NATIONAL LABORATORY
}

\author{
BIOLOGY DEPARTMENT \\ J. Walter Wilson, Acting Chairman \\ Brown University
}

Sanford S. Atwood

Cornell University

Sterling B. Hendricks

U. S. Department of Agriculture

\author{
Harland G. Wood \\ Western Reserve University \\ Raymond E. Zirkle \\ University of Chicago
}

\section{CHEMISTRY DEPARTMENT \\ Louis P. Hammett, Chairman}

Columbia University

\section{Ralph S. Halford \\ Columbia University \\ Joseph W. Kennedy \\ Washington University}

\author{
George B. Kistiakowsky \\ Harvard University \\ Joseph E. Mayer \\ University of Chicago
}

ENGINEERING DEPARTMENTS

Thomas B. Drew, Chairman

Columbia University

Harvey Brooks

Harvard University

John Chipman

Massachusetts Institute of Technology

Augustus B. Kinzel

Union Carbide \& Carbon Research Laboratories, Inc.

Philip Sporn

American Gas \& Electric Service Corp.

Alvin M. Weinberg

Oak Ridge National Laboratory

MEDICAL DEPARTMENT

G. Failla, Chairman

Columbia University
A. Baird Hastings
Harvard University
Colin M. MacLeod
New York University College of Medicine

\author{
Russell H. Morgan \\ The Johns Hopkins Hospital \\ Joseph T. Wearn \\ Western Reserve University
}

\section{PHYSICS DEPARTMENT \\ J. Curry Street, Chairman \\ Harvard University}

Samuel K. Allison

University of Chicago

Hans A. Bethe

Cornell University
Frederick Seitz

University of Illinois

Victor F. Weisskopf

Massachusetts Institute of Technology 


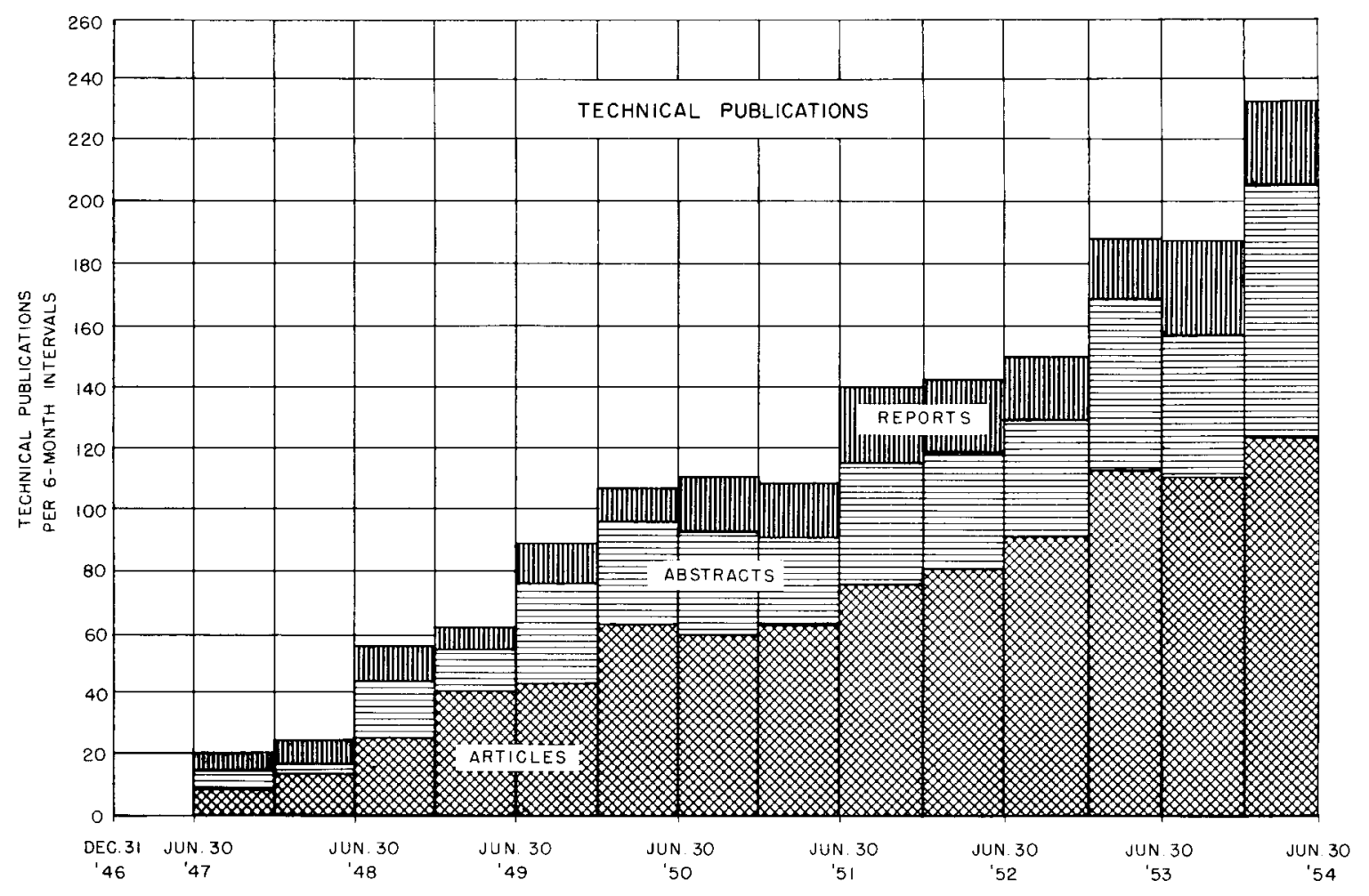

Figure 1.

for neutron time-of-flight experiments. A program for bringing an external beam into focus at a considerable distance from the cyclotron is under way. The 18-inch cyclotron has consistently produced a deflected beam of $1 \mathrm{ma}$ of $2.8 \mathrm{-Mev}$ protons in a target box. This larger beam current is also the result of improvements in efficiency and stability of the oscillator, the ion source and deflection system. Extensive measurements have been carried out on phasebunching of the proton beam with the view to adapting the extremely short duration beam-bunch to the measurement of neutron energies by time-of-flight methods with a precision of about 1 percent. The research electrostatic generator has been completely overhauled. This reconstruction program has been directed at increasing the upper voltage limit of the ion beam output, improving the general reliability and increasing the general experimental usefulness by miniminzing background radiations. The results have been most gratifying: beam currents of 20 to 30 microamperes are obtainable at a normal energy of 3.5 Mev while the $\mathrm{x}$-ray level beside the tank has been reduced by a factor about 100 times that previously in existence.

\section{NEW RESEARCH DEVELOPMENTS}

\section{ALTERNATING GRADIENT SYNCHROTRON}

While the high energy physics work of the Laboratory has been going forward at the Cosmotron, the development and design of even higher energy machines for eventual use in this research has become a significant part of the BNL accelerator program. Particles having energies far in excess of those available from the Cosmotron ( $3 \mathrm{Bev}$ ) or the Bevatron at the University of California Radiation Laboratory $(6 \mathrm{Bev})$ are available in cosmic rays and their utilization has been of inestimable value in studying nuclear particles themselves and the forces between them. These particles are, however, so few in number and the conditions of their existence so complex that quantitative experiments are often difficult or impossible. This situation is solved only by carry. ing out the experiments under controlled laboratory conditions made possible by high energy accelerators. History has shown the value of such machines and has confirmed the desirablity of going to ever higher energies. As noted last year, the application of the 
"strong focusing" principle makes the construction of accelerators producing protons with energies of a few tens of billion electron volts now economically feasible.

The alternating gradient principle (strong focusing) has found a wide acceptance in applications requiring high energy charged particle beams. Proposals for the construction of new accelerators embodying this concept have been received by the Atomic Energy Commission from several institutions. Also, the European Nuclear Laboratory at Geneva (CERN) is proceeding with the construction of a 25-Bev machine embodying these same principles. Brookhaven's scientists followed up their initial findings and considered the application of strong focusing to proton synchrotrons operating in the energy range of 10 to $100 \mathrm{Bev}$. Coordinated studies of problems associated with the design, development, and construction of 10-to-20-Bev machines were carried out at Princeton and jointly at Harvard and MIT under subcontract to Brookhaven. A group of midwestern scientists spent three weeks at Brookhaven during the summer of 1953 to study jointly the problems associated with the design and construction of such an accelerator for possible location at or near one of their home institutions. Their work has continued at their home institutions and elsewhere in the Midwest.

As a result of the studies carried out at Brookhaven and elsewhere, the Laboratory made a proposal to the Atomic Energy Commission in the fall of 1953 for the development, design and construction of a 25-Bev accelerator to be built on the Brookhaven Laboratory site. The total estimated cost of this accelerator, which has a magnet diameter of over 800 feet, was placed at twenty million dollars; this includes all costs for design and development as well as costs of construction of the accelerator itself, the necessary service buildings, and utility additions and modifications necessary to the project.

In January 1954, the Atomic Energy Commission announced its approval of the project. Funds were immediately made available to the Laboratory to proceed with the preliminary design of the accelerator, the service buildings and the necessary utility changes. In addition, funds were also made available for the Accelerator Development Division of the Laboratory to design and construct an analogue device to test certain principles involved in an alternating gradient machine and to gain experience in the handling of charged particle beams moving through fields of the alternating gradient type. It is expected that the knowledge gained in the study of phase transition and resonance problems with the analogue will permit design of a more efficient large proton synchrotron. The analogue machine will be 45 feet in diameter; it will accelerate electrons and will use electrostatic fields as guide and focusing lenses. Completion of the analogue is scheduled for early 1955 and results of operations are expected before the end of fiscal 1955.

During the spring of 1954, extensive discussions were held with a number of architect-engineer firms to find one firm capable of carrying out the design and the detailed engineering of all aspects of the project with the exception of the accelerator itself. The Stone and Webster Company of Boston was finally selected and a subcontract with the Laboratory was signed in the summer of 1954. Preliminary design to be followed by detailed design will start with the necessary additions to the site utility system and the construction of a service building for the AGS complex, which will be located on the northerly edge of the built-up area of the Laboratory. Over-all responsibility for development, design and construction lies with the Laboratory. To effect the proper management for carrying out this substantial task, the AGS Project has been set up as part of the Accelerator Development Division (to become a Department on July 1), with the Director of the Laboratory in immediate charge. Organizational details are summarized later in this introduction, along with the other organizational changes for the Laboratory.

\section{LIQUID METAL FUEL REACTOR}

In the field of reactor development the Brookhaven Nuclear Engineering Department a few years ago started a search for reactor components that would give the greatest ultimate promise for development of cheap electric power from fission heat. A reactor that would use a liquid metal solution or dispersion of uranium-233 as fuel and that would breed the uranium-233 from thorium appeared to have many desirable features. Such a liquid metal fuel reactor or LMFR might have such low capital cost per kilowatt and such low operating costs that the electric power could be produced at a price competitive with conventional fuels. Because the LMFR concept included unconventional materials and proc- 
esses on which engineering experience was lacking, a number of problems had to be solved -and still remain to be solved - before a large pilot plant reactor could be designed. Most of the development work on liquid metals has been carried out under restrictions of security and therefore has not been covered in these Annual Reports of the Laboratory. During the past year, however, seven papers on the LMFR and various component developments were declassified and presented at the First International Congress on Nuclear Engineering at Ann Arbor, Michigan in June. These papers were published as a group in the July 1954 issue of Nucleonics. Inasmuch as this is the first opportunity to present an over-all picture of these liquid metal fuel systems, it is appropriate to review the general concept at this point and to refer to the Engineering section of this report for further details.

Liquid fuel can be moved by pumping instead of requiring the elaborate handling equipment solid fuel elements need. It is not subject to radiation damage and it can be processed continuously for the removal of fission products; this eliminates many of the problems associated with the build-up of high intensity radioactive materials. The whole chemical processing system becomes an integral part of the reactor design. Because the fuel is a liquid metal it can produce high temperature steam and hence high thermodynamic efficiencies without the problem of a high pressure container for the core or blanket. A general schematic diagram showing the core heat exchanger, steam power generator and processing systems for both the core and the blanket is given in Figure 1 on page 41. (This is part of the Engineering Section). It should be emphasized that the description of the LMFR concept included in this report is not a design for a specific reactor but merely a scheme for developing a reactor design from the component studies that have been going on at Brookhaven.

It is obvious that it has been necessary to carry out many experiments in physics, metallurgy, heat transfer, and chemical processing in order to develop this very long range concept of an industrial power reactor. Much development work still needs to be done prior to the actual construction of a pilot plant unit. However, all indications to date are that the system as a whole has many desirable features not now available in other reactors under consideration, and that an LMFR experiment testing the concept and operation of all components as an integral ex- periment should be carried out within the next few years. Engineering studies, now contemplated, call for evaluation and feasibility reports by independent industrial organizations prior to such an experiment. An evaluation study is planned for the coming fiscal year. Up to the present time, the general philosophy of development has been directed at laboratory exploration of promising schemes for continuous processing of fuels and breeder materials and the use of materials which can provide high temperature steam under reasonable conditions of operation. Since Brookhaven was not heavily engaged in design of reactors for immediate application to military and production problems, it could take the necessary time to follow up promising avenues of approach even though the development processes were of a long range nature.

\section{CONSTRUCTION PROGRAM}

No major buildings or other facilities were under construction at the Laboratory during the report year. However, a number of minor projects were completed with most of the actual construction work being done under subcontract. The larger items in this category provided additional facilities or improvements to existing facilities, all of which are directly connected with the research program itself. With improvements of Cosmotron operations and increased beam intensity it was necessary to provide additional shielding at the Cosmotron. A $\$ 16,000$ enclosure was built up of large concrete blocks and slabs for the target section so that radiation levels in the control room and elsewhere were reduced to nominal values.

The use of high energy beams from the Cosmotron necessitated the provision of a number of large electromagnets for purposes of analyzing those beams and deflecting the charged particles to experimental equipments at remote locations. It has also been necessary to provide motor generators and control equipment to power the magnets properly. The construction of the power room as an addition to the Cosmotron and the installation of the associated electrical equipment was completed at a total cost of approximately $\$ 100,000$. A counting room $(\$ 25,000)$ was added to the chemistry complex in order to accommodate increased work resulting primarily from the successful radiochemical projects associated with the bombardment of targets by high 
energy beams from the Cosmotron. A $\$ 20,000$ facility was added at the reactor to provide on-the-spot decontamination of tools and equipment used in and around the reactor.

The major facilities such as the reactor and the accelerators are housed in structures of a permanent type, designed and built specifically to meet their needs. The Biology laboratory is the only general research laboratory that has permanent housing. Several semi-permanent structures built by the Army are occupied by research units and services units. However, most of the research laboratories are located in temporary wooden structures which were modified from their former Army use.

The long range development of the Laboratory plant with eventual replacement of temporary structures by more suitable and permanent structures has been under continuing study by the Laboratory, the Board of Trustees, and the Commission. A Master Plan has been presented to the Commission as a guide. These recommendations call for the ultimate construction of permanent buildings for the research laboratories and the service and administrative units, to take place over a 12-to-15 year period. The location of the new units will provide for consolidation of the site and a redistribution of research laboratories in order that physics, chemistry, electronics and engineering units will be in close proximity to the accelerators and to the reactor and that biology and medicine will be near to each other. The permanent Medical Center with its research laboratories, research hospital, nuclear reactor especially designed for medical use and industrial medicine clinic have been undergoing preliminary design during the past year. In the spring of 1954 , a preliminary design study based on requirements and general specifications set by the Brookhaven Medical Department and Architectural Planning Division was undertaken by the firm of Eggers and Higgins. Their final report along with preliminary drawings and specifications was presented to the Commission in support of the FY 1956 budget request for an appropriation for the Medical Center. It is hoped that it will be possible to proceed with detailed design of the Medical Center starting in January 1955 so that construction may proceed early in fiscal 1956. The construction of the Medical Center will be a realization of the first of the Master Plan buildings.

The construction of the alternating gradient synchrotron on the northerly side of the present built- up area has changed the plan for the location of a permanent physics laboratory and engineering laboratory from that contained in the Master Plan. The new high energy accelerator will be so situated that the most logical and appropriate location for the permanent physics laboratories is that now occupied by the temporary nuclear engineering laboratory. Having the physics laboratory in this location will provide a natural departmental headquarters midway between the Cosmotron and the 25-Bev accelerator. However, this requires that permanent engineering laboratories be built first so that the present temporary engineering building can be pulled down to make way for the physics laboratories. Changes in the general engineering program and a recognition of the value of the existing Hot Laboratory for chemical engineering work have given rise to a revised concept of the permanent engineering laboratory. It is now felt that the most appropriate structure for such a laboratory would be an addition to the present Hot Laboratory and that the more extensive permanent laboratories originally considered are not required. In a similar fashion, the advent of the AGS project has somewhat reduced the laboratory and office requirement for physics. As a result, the present plan, which will be formally presented to the Commission, calls for early construction of engineering laboratories to be integrated with the Hot Laboratory to be followed immediately by provision of a central physics laboratory conveniently situated with respect to both the Cosmotron and the 25-Bev accelerator. A formal request for funds to go forward with construction of the engineering building in FY 1956 was submitted by the Laboratory; however, the Commission elected to delay budget action until after FY 1956.

\section{COOPERATIVE RESEARCH}

One of the original objectives in establishing Brookhaven National Laboratory was to make available to qualified scientists from universities and other institutions, facilities which were not normally available to them at their home institutions. During the early years of Brookhaven's existence there was little outside participation in the Brookhaven program since the Laboratory was undergoing a period of construction and major facilities were not available. Also, as each facility came into use, a reasonable pe- 
riod of time was necessary for Brookhaven scientists themselves to become acquainted with the use of that facility, to be able to advise others in effective programs of research which could be carried out on a cooperative basis. It is most gratifying to report this year that the cooperative work at Brookhaven has increased by substantial amounts and that research programs made available through the cooperative use of Brookhaven's facilities are now in existence in a large number of locations.

One type of cooperation is the provision of services by Brookhaven on the Brookhaven site and the shipment of materials to the investigator's home institution for him to consider as part of his regular work. The use of isotopes produced by the nuclear reactor and by the cyclotron are prime examples of this particular type of service. In addition, neutron irradiation services are available from the reactor and gamma irradiation services are available from reactor-irradiated cobalt. Brookhaven's establishment of several multi-kilocurie sources in a gamma irradiation facility has provided a new avenue of research and study of special interest to industrialists. Cobalt gamma sources, similar to those being used for service irradiation in the study of initiation of chemical processes and other chemical effects, are also used in the Brookhaven gamma field in an extensive program on somatic mutations in plants. As noted in Figure 2 the gamma field work on somatic mutations involves 11 different educational institutions and several agricultural experiment stations associated with both state and federal governments. Experiments in plant physiology and in genetics also obtain their basic material for study from irradiations performed in this gamma field.

The major advance in cooperative research at Brookhaven's facilities has come about in the high energy physics program through use of the Cosmotron.Two major types of cooperative work have been undertaken. In one instance, after joint planning, the experiment is carried out by Brookhaven scientists and the record of the experiment is then taken to the investigator's home institution, for an analysis by him. Typical of this type of work are experiments run with BNL cloud chambers, with the pictures provided to the cooperating scientists for analysis and study. Similar experiments have been carried out with nuclear emulsions in which the experiment is designed in cooperation with the visiting investigator, the exposure is made at BNL and the study of the nuclear emulsion is then done at the participating institution. On the other hand, several visiting scientists have actually brought to Brookhaven cloud chambers provided by their own universities, have set them up at the Cosmotron and operate complete experiments.

Neutrons from the reactor are available for onsite experiments. As at the Cosmotron, visiting scientists may provide their own equipment or use Brookhaven equipment to carry out their studies. This is done in cooperation with an interested research department at the Laboratory; the project is, of course, one which fits into the general research interests of the Brookhaven program. Typical of such work is the establishment of diffraction spectrometers at the reactor which are used, for example, by the General Electric Company for basic studies in solid state physics, and by Columbia as part of a cooperative neutron cross-section program for the Atomic Energy Commission. Experiments go on continuously at the reactor for companies carrying out both studies of fundamental interest to themselves and studies which are sponsored by the Atomic Energy Commission or other government agencies.

Cooperative research in the life sciences field can, in general, trace its origin back to the use of the reactor in some fashion. As noted above, the extensive use of the high intensity cobalt as a source of gamma radiation and the use of isotopes are prime examples. In addition, it should be noted that the direct use of neutrons is playing an ever-increasing role in biological research and in medicine. Many institutions are taking advantage of the neutron effects produced in biological materials, especially in plant breeding and in genetics. Since it is Brookhaven's belief that the eventual commercial development of a new species, e.g. a rust-resistant oats or the preservation of potatoes through gamma irradiation, should be carried out by other institutions more closely associated with agricultural and economic problems, it is quite proper that extensive cooperative arrangements be made with these institutions in the first instance and that they be provided with facilities to implement their own programs of study. The use of the thermal column at the nuclear reactor has involved some 24 educational institutions and 10 government agencies alone.

One of the major areas of study in the Medical Department is neutron capture therapy and the particular disease state now under consideration is glio- 


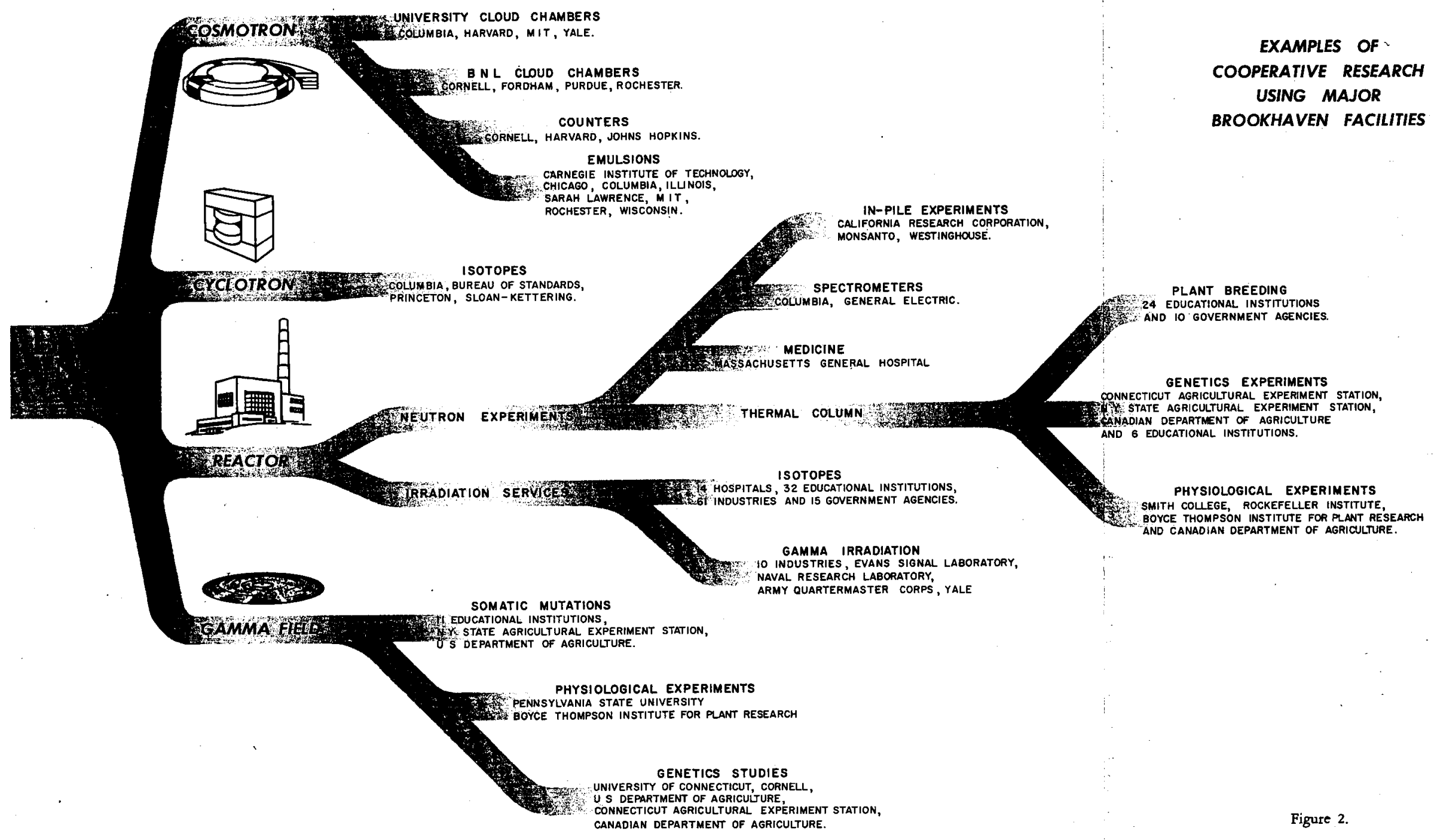


ASSOCIATED UNIVERSITIES, INC.

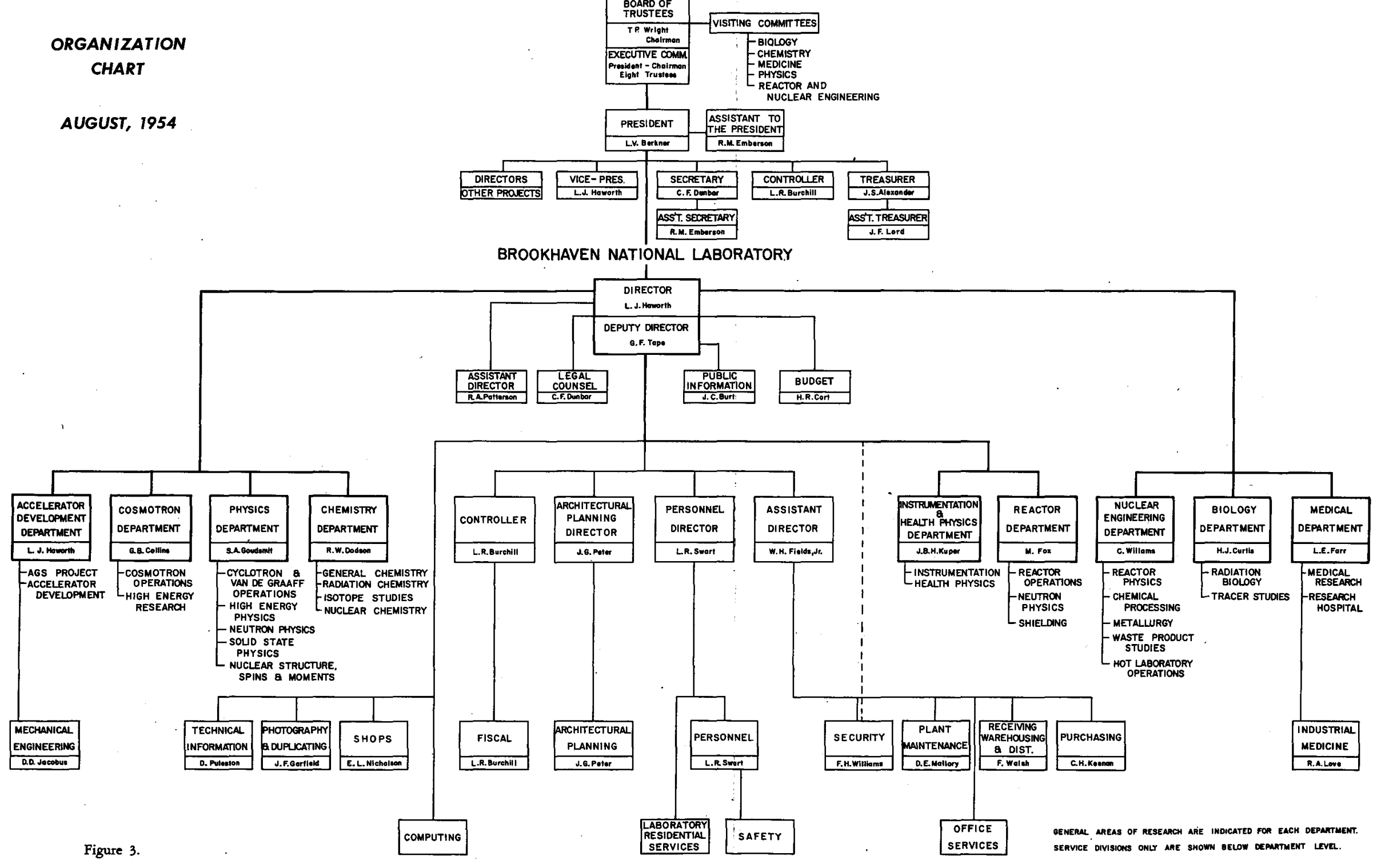


blastoma multiforme, a particular type of brain tumor. It is only through cooperation with other medical institutions that a problem as enormous as this can be completely attacked through the use of the special techniques and capabilities of those institutions in conjunction with Brookhaven's special facilities. Here again the reactor as a neutron producer has played an extremely vital role.

No attempt has been made to add up the total numbers of scientists or institutions involved in the ever-growing program of cooperative research at Brookhaven. Reference is made to Figure 2 in which specific instances of cooperation for purposes of example only are listed. The figure is not meant to be all-inclusive.

\section{RESEARCH ACTIVITIES}

Although the research program of each department is representative of its own interests, the particular facilities and equipment available to it and the capabilities of its staff, the program of the entire Laboratory may be divided into four categories:

1. Fundamental studies of atomic nuclei, the particles which constitute them, and the forces involved in their structure;

2. The physical, chemical, and biological effects of radiation;

3. The use of nuclear tools, such as neutrons, charged particles, gamma rays, and isotopic tracers, in all branches of scientific research;

4. Research and development, not necessarily of a nuclear nature, directed at the specific problems of atomic energy development.

The status of the research activities and the important developments of the year for each department of the Laboratory can be found in the separate departmental sections of this report. A few samples of research in progress and of significant advances made in the above categories will be noted here.

\section{FUNDAMENTAL STUDIES OF NUCLEI}

Studies of atomic nuclei, the particles which constitute them and the forces involved in their structure are carried out in the Physics Department, the Cosmotron Department and the Chemistry Department. The study of nucleon-nucleon interactions and the products of those reactions and the study of the properties and behaviour of the unstable particles such as mesons and hyperons (heavier than protons) which may be produced are carried out by the physicists. Their tools consist of counters, cloud chambers and photographic emulsions. The radiochemists study the nuclear fragments produced by the bombardment of a target nucleus with high energy particles. In order to identify the individual products and thus work back to an understanding of the mechanism of the reaction, the chemist concentrates on the nuclear fragments while the physicist concentrates on understanding the behaviour of the individual nucleons and the various unstable particles produced in their interactions. Studies of atomic nuclei at Brookhaven, of course, extend from the range of energies available with the Cosmotron down to essentially zero energies at which the static properties such as spins and masses of the nuclei are measured. However, the greatest proportion of the work and the most significant changes in relative emphasis over prior years have occurred through the full and continual use of the Cosmotron for the production of high energy particle beams.

Protons from the Cosmotron can produce all the well established varieties of heavy mesons and hyperons. The numbers of these heavy particles is not so large that "beams" have been produced; however, an appreciable number of events has been observed. The earlier spectacular observations are being displaced by detailed quantitative experiments providing better data and improved statistics of observation. Counter experiments on the total cross sections for the scattering of $\pi$-mesons take advantage of the Cosmotron's ability to produce meson beams ranging in energy from 0.2 to $2.0 \mathrm{Bev}$. Other experiments with counters involve the measurement of interactions of high energy neutrons, protons and mesons with similar nuclear particles or simple nuclei.

The chief advantage in the use of cloud chambers for the observation of high energy events is that the interaction of the high energy particle takes place with the known gas in the chamber under conditions where unambiguous interpretation is often possible. Again nucleon and meson beams are used. Mostly pions are produced in these interactions, but 2 number of events showing heavy unstable particles has also been observed. Studies of this type are aided by two types of cooperative work: 1) the study and analysis of cloud chamber pictures taken with BNL equipment by cooperating scientists at their home in- 
stitutions, e.g., Cornell, Duke, Fordham, Purdue, Rochester, Yale and the Naval Research Laboratory; and 2) the carrying out of complete studies with chambers built at their home institutions and moved to Brookhaven for use with Cosmotron. Yale, Harvard, Columbia, and MIT scientists have set up such equipment.

Photographic emulsions have been successfully used to reveal the existence of artificially produced heavy mesons and hyperons and to contribute to an understanding of their properties. It was not known from cosmic ray observations how energetic protons must be to produce heavy mesons; it was not certain that heavy mesons could be produced at Cosmotron energies. Consequently, the first efforts with the machine were to find out whether k-mesons could actually be created with the Cosmotron. Both positive and negative k-mesons have been created by collisions of 2.2- and 3-Bev protons with beryllium and copper targets. It was established that their masses were about 1000 times the mass of the electron. It has further been shown that for the positive k-mesons the decay process is a three-body one which produces mu mesons. The study of decay processes and measurement of masses is continuing. Plates exposed under varying conditions at the Cosmotron are sent to the participating universities for their study and analysis. A group at the University of Wisconsin, for example, which is studying emulsions exposed to the internal proton beam, has found some 14 examples of the so-called unstable fragments. These are believed to be nuclear fragments in which a neutron has been replaced by a hyperon, which in a very short time decays into charged particles.

A substantial portion of the Chemistry Department's efforts in nuclear chemistry has been devoted to studies of proton-induced nuclear reactions with the Cosmotron. Measurements of formation cross sections of about 50 radioactive nuclides produced in the bombardment of copper with 2.2-Bev protons are nearly complete. The striking difference between these results and those of corresponding studies at lower energies ( $340 \mathrm{Mev})$ is that the production of nuclear species within a few mass numbers of the target has become relatively less at the higher bombarding energy while the loss of 15 or more nucleons is much more probable. Studies of this type indicate that a high probability of large energy transfers can not be accounted for by nucleon interactions alone. A plausible mechanism for imparting hundreds of
Mev of excitation energy to a nucleus is the creation and subsequent scattering and reabsorption of a meson all within the same nucleus. Studies of the products resulting from similar bombardments of lead and bismuth have led to similar conclusions with the most probable products some 20 mass numbers below the targets. A somewhat surprising result from the lead studies shows a relatively high cross section for the formation of light elements. The better understanding of the processes involved in nuclear reactions observed at these energies and the establishment of possible correlations with meson processes make it necessary to study the variation of cross sections with bombarding energy. Therefore, excitation functions have been determined for a number of the fragments formed. This radiochemical approach to the understanding of high energy phenomena has yielded enormous data and can contribute substantially more. As noted in last year's report the production of significant quantities of radioactive species by proton bombardment at the Cosmotron exceeded all expectations and has permitted the very rapid and exhaustive research program in this area.

The uses of neutrons in the studies of atomic nuclei range from the conventional cross-sections measurements which give a measure of the interaction of the neutron with other material, to studies on the properties of the neutron itself. The determination of neutron cross sections is a continuing program and measurements are being made at all energies with a variety of machines and equipment. These include the slow chopper, the fast chopper and crystal spectrometers at the nuclear reactor and time-offlight equipment used in conjunction with both the 18-inch and 60-inch cyclotrons. The fast chopper has provided a tremendous amount of new information through its ability to detect and resolve levels in energy regions which previously could not be properly measured through lack of appropriate equipment and techniques. Considerable progress has been made in the development of efficient neutron detectors as well as improvements in the operation of the chopper itself to provide a higher resolution. Details of the resonances of many materials have been noted and have been incorporated with other cross-sections measurements made at Brookhaven and elsewhere in the continuing compilation of neutron cross-sections information published by the Brookhaven group in cooperation with the Atomic Energy Commission's Cross-Sections Advisory Committee. 
Physicists from Harvard and Brookhaven have collaborated in a high precision measurement of the ratio of the magnetic moment of the neutron to that of the proton. A beam of neutrons from the reactor was allowed to impinge at small glancing angle on a polished cobalt mirror which was magnetized. By analysis of the reflected neutrons, through the use of magnetic fields and further reflection, it was possible to compare the magnetic moment of the neutron to that of the proton to an accuracy of a few parts per million.

The detailed measurement of neutron resonances yields information about the nucleus which permits correlation with various theories of nuclear models. Another approach involves a detailed study of the radiations emitted by unstable nuclei which thereby permits a description to be made of the various excited states of the nucleus in terms of the energy levels, types of radiative transitions and other nuclear parameters. Important progress has been made in the interpretation of decay schemes and excited levels of several nuclei and in the measurement of very short-lived isomers. This progress has been made possible through very ingenious application of existing techniques and their refinement and adaptation to each particular case under investigation. Previously, spins of isomers have been predicted from information gained through a study of the decay scheme. For the first time, the direct measurement of the spin of an isomer, namely calcium-134, has been made. The result of this measurement was confirmed within a few hours by a separate group studying the decay scheme of the same isomer.

\section{EFFECTS OF RADIATION}

Radiation can be used to study the effects of the radiation itself on materials and organisms, or to induce effects in specimens to permit their study under conditions not otherwise possible. For example, radiation damage of a cell may give rise to information on radiation effects, but a study of the damaged cells may lead to an understanding of cell development. The physical, chemical and biological effects of radiation are all under investigation. Materials are being studied both for changes in their fundamental physical properties, such as thermal conductivity or electrical resistance, under neutron radiation and for their engineering application as suitable materials for use in high neutron and gamma radiation fields. Metallurgical studies are devised to satisfy the requirements of various reactor designs and associated equipment. Extensive studies on the behavior of graphite and of various alloy materials of special interest to the Brookhaven Liquid Metal Fuel Reactor program, under varying conditions of irradiation, are underway.

Changes in the physical properties of materials resulting from irradiation are being studied with continued emphasis on effects produced in alloys. For example, an irradiation of one week at $50^{\circ} \mathrm{C}$ in the reactor causes a drop of a few percent in the electrical resistivity of the alpha brasses. Such effects are being studied at liquid helium and liquid nitrogen temperatures and at room temperature in order to isolate various contributions to the resistivity. $\mathrm{Ra}$ diation effects in insulators are also under consideration. Particular oxides have demonstrated changes in optical adsorption which are unstable at room temperature. Changes in the optical adsorption of aluminum $\mathrm{Al}_{2} \mathrm{O}_{3}$ have been produced by both gamma and neutron irradiations. These effects are being interpreted in terms of the lattice properties of the solid. The effect of radiation upon explosives has been investigated as a cooperative undertaking with Picatinny Arsenal. Work on azides has begun with preliminary experiments showing marked color changes which differ for gamma and neutron radiations. Preliminary $\mathrm{x}$-ray studies indicate that the structure of sodium azides, for example, is extremely sensitive to irradiation and less sensitive to thermal and mechanical treatment.

Radiation chemistry studies are concerned with chemical changes produced in water and aqueous solutions when they are exposed to ionizing radiations. The modes of chemical decomposition of water by gamma rays have been considerably clarified and detailed during the year. Radiation decomposed water into molecules of $\mathrm{H}_{2}$ and $\mathrm{H}_{2} \mathrm{O}_{2}$ and free radicals $\mathrm{H}$ and $\mathrm{OH}$. The radicals being unobservable directly, their existence and number must be inferred from chemical changes which added materials undergo. Specific results have been obtained for the gamma ray yield of molecular hydrogen from water and it is to be noted that this yield is independent of the nature and concentration of added solutes (at least up to $.003 \mathrm{M}$ ) and practically independent of temperature. Molecular products are believed to originate in the densely ionized "hot spots" formed by the tracks of slow secondary electrons. A test of this 


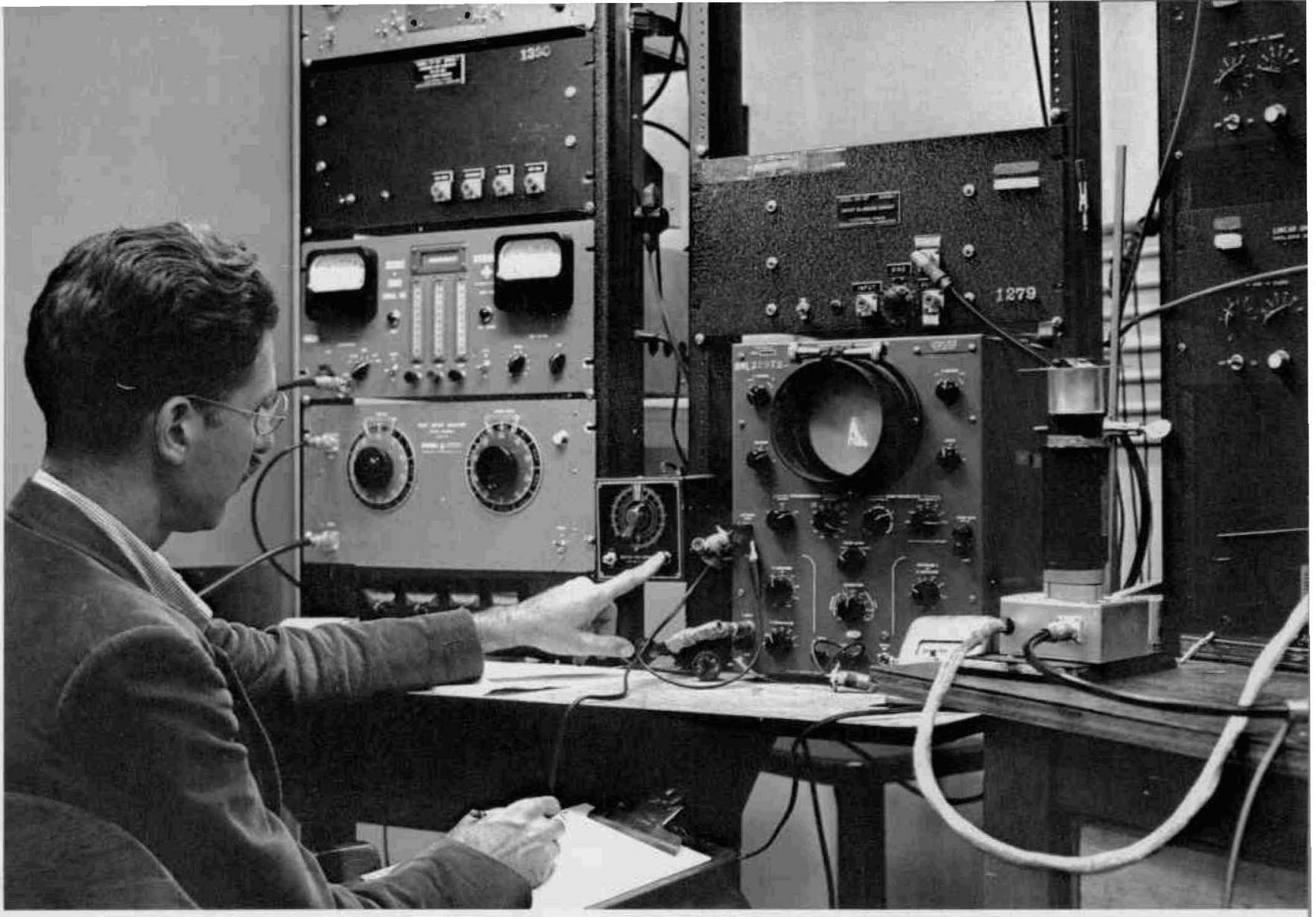

Figure 6. The spectrum of tellurium-123 appears on the scope and gives information about the low level excited states of its nucleus.

Figure 7. The 60-inch cyclotron is used to study models of waters decomposition under irradiation. In the picture the liquid target is inserted into the deuteron beam.

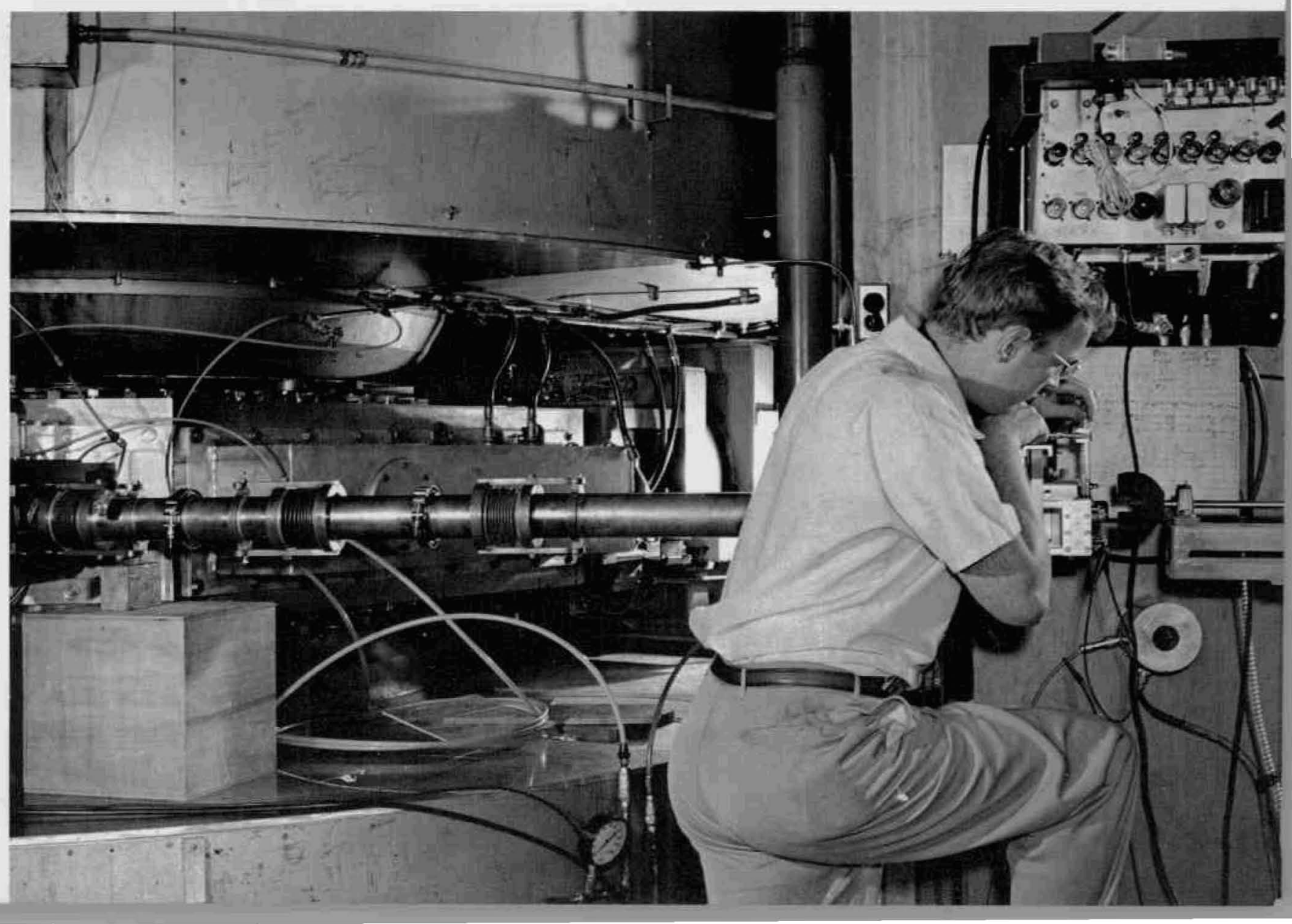


hypothesis can be made through the variation in density of the "hot spots" by the use of different types of radiations and the use of ionizing particles of different velocity. The use of ionizing radiations from the cyclotron for this study is now under way.

The use of ferrosulfate solution as a standard for gamma ray and hard x-ray dosimetry has become almost universal among radiation chemists. The measurement of the absolute yield has an importance far beyond radiation chemistry and has provided the only practical check on the absolute accuracy of electrical methods of gamma ray dosimetry. Determination of the absolute value of the ferrosulfate yield has been completed. The result is in very good agreement with the latest calorimetric values obtained elsewhere but the accuracy is not much better than $\pm 3 \%$. These measurements will be extended to improve the precision.

Studies of the biological effects of radiation range from a study of simple cells to applications to complete plant and animal organisms. The facilities used by the biologists include $x$-rays, gamma rays, and both thermal and fast neutrons. The gamma field, a 6-acre growing field containing a high intensity cobalt source (over 2000 curies) of gamma rays, continues to be extremely valuable. The abnormalities of the plants grown in the field are under continuing study and will be for a number of years. The thermal neutron facility at the reactor has received extensive use throughout the year. A number of interesting reactions to radiation have been reported for plants and more continue to be found and studied.

Each plant species appears to have a characteristic reaction to radiation. One of the most interesting observations made in this program is that certain tobacco plants which normally exhibit only a few spontaneous tumors develop enormous numbers of tumors when exposed to radiation. In some cases, almost the entire plant appears to be tumor tissue. Although it has been known for many years that radiation produced tumors in animals this is the first clear case of radiation-induced carcinogenesis in plants. There is a substantial amount of work in progress on attempts to find the effect of different radiations on cells and cellular growth. Essentially this is a comparison of the difference in biological effect between thermal neutrons and $x$-rays. It has now been found that differences which exist between $\mathbf{x}$-rays and thermal neutrons as measured by growth response of seedlings are very much dependent upon the gaseous atmosphere of the seeds and upon the water content of the seeds. For fast neutrons the sensitivity increases with increasing water content, probably because of the higher hydrogen concentration available for recoil reactions. Changes in cellular damage are being followed for all these radiations and it is interesting to note that chromosome damage is by no means parallel to the observed inhibition of growth. In general, it can be said that neutrons cause relatively more damage to the nucleus for a given growth inhibition than do $\mathrm{x}$-rays. The mechanism by which radiation causes changes in mammals is very poorly understood. One theory is that there is a toxic substance formed in tissues by the radiation which enters the blood stream and causes the general radiation responses. In the past, attempts to find this toxic agent have proved futile. The attempts have usually been made by injecting blood or serum from an irradiated animal into a normal animal and then looking for radiation effects in the recipient animal. No effects have been observed. Recently, adrenalectomized mice were injected intravenously with serum from the irradiated adrenalectomized rats. This serum will cause the death of these mice when given in amounts as small as $0.2 \mathrm{cc}$ and when taken from the irradiated rat 24 hours post-irradiation. The chemical nature of this toxic factor is not yet known except that it is very labile, being destroyed if the serum stands 30 minutes at room temperature.

In medicine, radiation is used both externally, with neutrons from the reactor and gamma rays from cobalt sources, and internally with sources such as internally administered isotopes or in vivo induced disintegrations. A large portion of the medical effort is devoted to the development of neutron capture therapy. This procedure is still being confined to patients with proven malignant brain tumors of the type called glioblastoma multiforme. The original series of treatments carried out in 1951 and 1952 indicated that the general type of treatment was promising and that a radiation effect existed, but that the neutron dose delivered to the tumor was of marginal benefit. Several possibilities existed such as increasing the neutron flux and increasing the concentration of boron in the tumor. An extensive program involving irradiation of mice to determine biological effects of this type of treatment and radioautographic studies of boron-containing tissue slices in contact with nuclear track emulsions has been carried out. 
Also, an extensive modification of the facility at the reactor was completed which permitted irradiation treatments to be made through the use of a shutter in the pile and also provided increased flux over that available for the previous series of measurements. Four patients have been treated in the current series but results can not be assessed at this early date. The use of short-lived isotopes has also received considerable attention, both in research and therapy, and is a significant feature of the Brookhaven medical program.

\section{USE OF NUCLEAR TOOLS}

The tools of nuclear and atomic science are in use by all research workers at the Laboratory, generally in the form of tracers of radioisotopes or of various radiations. The major portion of this research effort is in solid state physics, chemistry, and

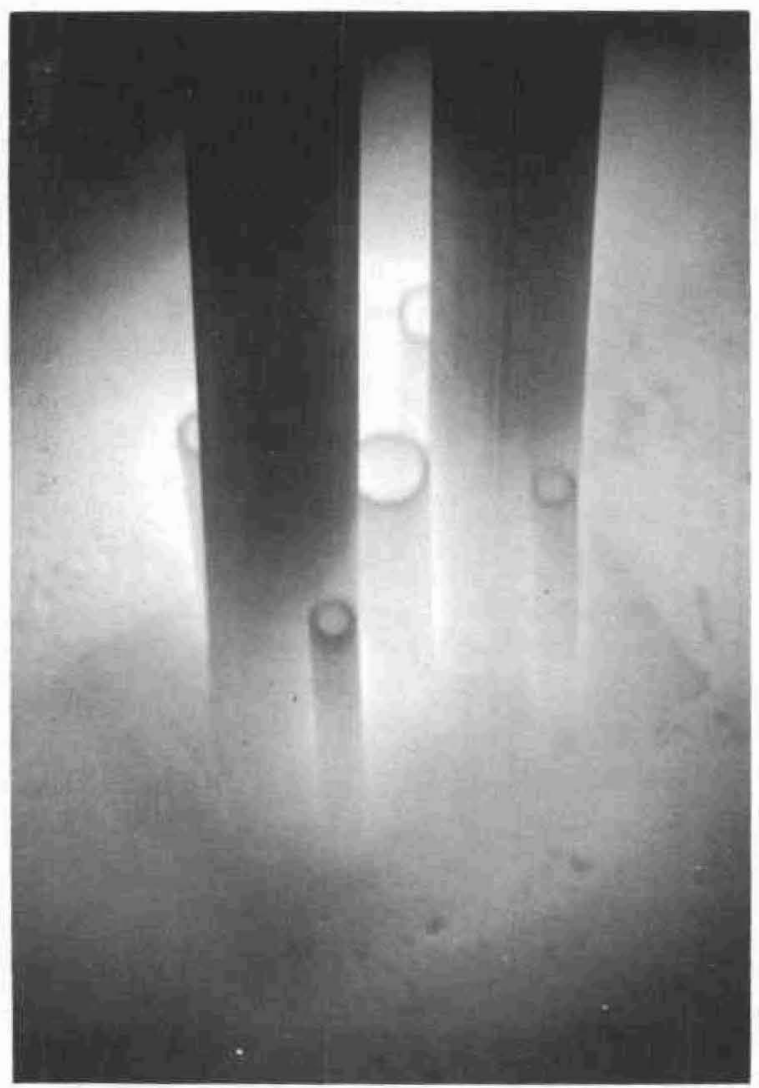

Figure 10. Underwater gamma irradiation facility using high intensity (thousands of curies) sources. The tube sources are seen by Cerenkhov radiation (glow) in the water surrounding them. Samples to be irradiated are introduced into the two large pipes from above. the life sciences. Neutron diffraction studies of structure, the kinetics of chemical reactions, photosynthesis, metabolism, protein synthesis, and the initiation of chemical reactions, and medical diagnosis and therapy are but a few examples of the applications of tracers or radiation to general scientific problems. The production of gamma sources of several thousand curies source strength, made by the irradiation of cobalt and tantalum in the nuclear reactor, is continuing at a high level. The potential capacity of the Brookhaven reactor for the production of radioactive cobalt is approximately 20,000 curies per year. There is an increasing demand from other institutions for sources with which to study chemical reactions, sterilization of foods and pharmaceuticals.

The use of radioisotopes and the application of radiation as a tool has its greatest use in the hands of the life scientists. The somatic mutation program utilizing the gamma field has completed its second year and many exposed specimens have been returned to the cooperating institutions for study. The radiation is used to accelerate the mutation process. This is a great aid in plant breeding. Studies of the relative effectiveness of $\mathrm{x}$-rays and thermal neutrons have indicated in the case of barley and also of corn that more than twice as many mutations can be produced by thermal neutrons as by $\mathrm{x}$-rays and still the plant will produce seeds. As a result of this work an attempt was made to see whether it would be feasible with a relatively smaller amount of work to produce and isolate a given mutation. A variety of oats was tested to see if a mutant could be produced which would be resistant to a particular strain of oat rust. Seed was irradiated with thermal neutrons and the first generation was grown and seed collected to make sure the character which was being dealt with would be properly propagated genetically. The second generation was grown and when the plants were almost matured, the entire field was infected with the oat rust. Forty-nine plants were found to be resistant. Seeds from these resistant plants were grown again and it was found that in all cases the character bred true. This demonstrates that it is possible in the course of about 18 months to develop a plant resistant to a particular disease but in all other aspects identical to the parent variety. This might well have taken at least ten years by conventional methods. The application of radiation for the development of desired mutants is being continued. The final development of the seed for com- 
mercial application is left to the agricultural experimental stations and others.

The study of the distribution of radiomanganese in rat tissues showed that high concentrations of manganese occurred in the liver and pancreas. Calculations indicated that if an isotope with a suitably short half-life were chosen, heavy radiation could be administered to these organs without undue effect on other tissues. The application of a short-lived 2.6hour manganese isotope was selected for admistration to two patients suffering from carcinoma of the liver. This early application of a potential therapeutic isotope resulted from the study of manganese distribution. The preliminary results from the first two treatments warrant further investigation.

Structural studies of solids by means of neutron diffraction techniques have been concerned with the general fields of single crystal anaylsis of ferroelectric hydrogenous crystals, arrangement and dynamics of the magnetic spin system in ferromagnetics, and studies of the deviations from perfection of crystal lattices, by slow neutron transmission. Neutron diffraction techniques as a supplement to $\mathbf{x}$-ray and electron diffraction techniques have received wide application. However, such work can be carried out at only a few installations in the country where neutrons are available. In addition to studies in the Brookhaven Chemistry Department on the structure of various ferrites, cooperative work is under way between Brookhaven and Pennsylvania State University physicists on Rochelle salt in the ferroelectric phase. Neutron data are taken at Brookhaven. The heavy atom positions have been studied elsewhere by $\mathrm{x}$-rays. Through correlation of neutron data with such $\mathbf{x}$ ray data, the lattice arrangements for the hydrogen atoms can be determined. A number of related studies mostly on magnetic diffraction have been carried on by a group from the General Electric Company and by members of Physics and Chemistry Departments. The Bell Telephone Laboratories plan to use a spectrometer arrangement similar to that of $\mathrm{G}$. E. at the reactor in the very near future.

\section{RELATED RESEARCH AND DEVELOPMENT}

Research and development in areas which are not in themselves nuclear or atomic but which are vital to the development of the atomic energy program are carried out primarily by the engineering departments. A wide range of studies is pursued: for ex-

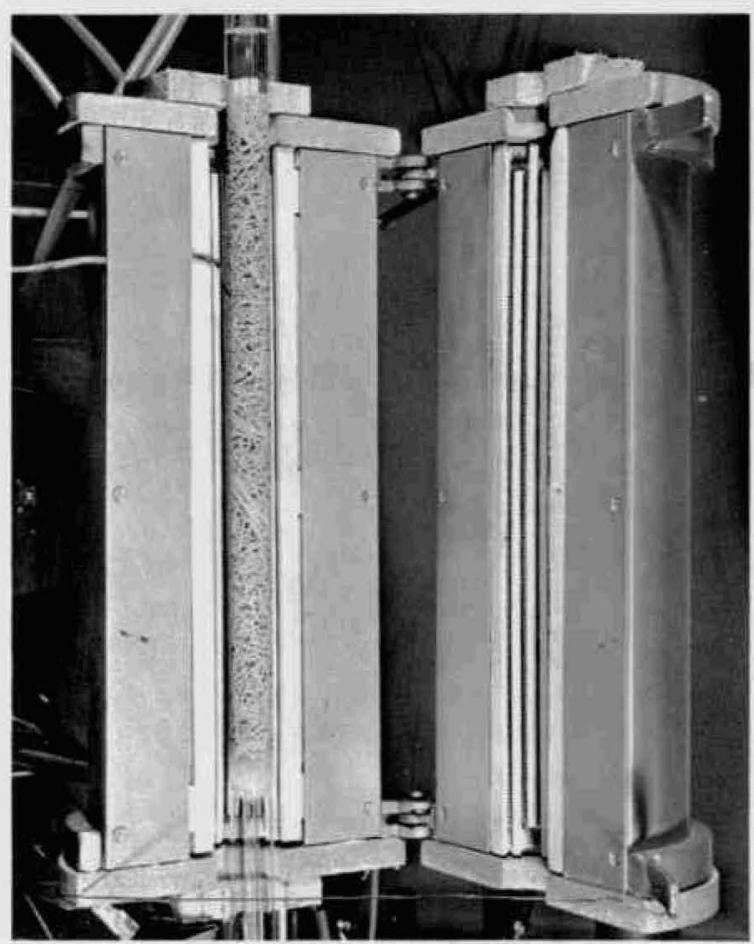

Figure 11. A process for high level radioactive waste involves the adsorption of fission product ions on montmorillonite clay and the fixation of the adsorped ions by heating the clay to a high temperature. The picture shows extruded clay in a column used for contacting the clay and the waste solutions.

ample, basic chemical studies of those elements associated with fission processes, metallurgy of reactor fuels and container materials, heat transfer and instrumentation. Many of these studies are related to reactor components and the design of complete reactor systems. The concentration of liquid radioactive waste materials is being studied in order that the bulk of the liquid material can be disposed of by ordinary means and a concentrated radioactive residue can then be fixed for ultimate disposal. The progress in this area has been directed at the development of a calciner process in which the active material eventually becomes part of an anhydrous melt and solidifies into cake form after the inactive solvents are in effect evaporated away. Continued development of this process is necessary. Another approach directed at devising a system for the ultimate disposal of radioactive wastes makes use of the adsorption of fission product wastes in clays and their eventual fixation through baking of the clay. Satisfactory results have been obtained and a pilot process plant is under construction. 
The field of electronics is vital to the further development of atomic energy. The understanding of nuclear processes requires the provision of higher speed circuits and detection devices. Development at Brookhaven has been concentrated on data-handling equipment and on instrumentation for magnetic measurements. As research projects and their instrumentation become more complex, data-taking becomes a serious problem. The use of electric typewriters for automatically printing out accumulated data has been tried. Another system records data from crystal spectrometers on business machine punch cards for automatic processing with the BNL computer.

\section{BUSINESS AND ADMINISTRATION}

Significant changes in the business and administrative operations of the Laboratory occurred during the report year. One of the most extensive changes involved the installation of punch card equipment in the Fiscal Division. An extensive survey had been made during the previous year to study the application of such a system to fiscal operations and to evaluate the possible contribution such a system might make to a scientific computing program. It was demonstrated that fiscal requirements alone could justify the installation of the necessary equipment. Furthermore through the provision of a slightly more elaborate machine, some of the Laboratory's scientific computing work could be handled locally through a scientific computing group using this same equipment.

The Remington Rand punch card equipment was installed in the Fall of 1953 and its application to Laboratory problems was first made January 1, 1954. In the month of January, material and supplies inventory records, accounts payable and all payroll work was placed on the punch card equipment. In the month of March, all bookkeeping, with the exception of general ledger work, was added to the mechanical system. Personnel records and fixed asset records will soon be added. The scientific computer group was established with a mathematician-programmer and equipment specialist and a computer. The program of scientific computation moved forward rapidly with application to problems arising from work in nuclear engineering, physics, chemistry, accelerator development, medicine. Calculation of neutron cross sections and reduction of data obtained from exponential experiments on reactor assemblies were the first problems handled by the computer.

In addition to the above changes in business operations, striking improvements which had been initiated in the previous year continued. The gains effected through the continued cooperation of Inventory Management, Purchasing, and Receiving, Warehousing and Distribution were reflected in improved service for the customer and in a remarkable reduction in active stores inventory. The large effort involved in the disposition of excess inventory which had taken place over the last two years diminished by the close of the fiscal year. The opening total inventory (exclusive of precious metals) July 1, 1951 amounted to almost $\$ 1,000,000$; the closing inventory, June 30, 1954 was approximately $\$ 400,000$, with no excess awaiting disposal. The maintenance of active stores requires continuing scrutiny and the weeding-out of obsolete and dead items. This process is continuing and its success over the past few years can be noted from the fact that the number of months' investment in active stores inventory has dropped from 10 months in FY 1952 to $61 / 2$ months in FY 1954. This maintenance of a more active stock has at the same time been accompanied by a greater use. A steady increase of issue by delivery from central stores and from branch stock rooms is taking place rather than the over-the-counter issue to the user.

The greatest number of purchase orders in the Laboratory's history was written during the year: nearly 16,000 orders for a total value of approximately two and a half million dollars. This total dollar figure is exceeded only by FY 1948 and FY 1949. Considerable effort has been spent during the past year in making use of excess property available from other AEC contractors. Approximately $\$ 200,000$ of new or slightly used equipment and material was acquired for a cost to the Laboratory of about 38 percent of its original value plus transportation.

No changes in Laboratory organization took place during the report period; however, several important changes will go into effect in July and August, 1954. These changes are reported here and the chart Figure 3 reflecting the Laboratory organization as of the date of these changes has been included as part of this report to show the latest status. The Accelerator Development Division of the Laboratory becomes the Accelerator Development Depart- 
ment on July 1, 1954, with Dr. Leland J. Haworth, the Director of the Laboratory, acting as Department Chairman. Dr G. K. Green will serve as Deputy Chairman, M. M. Small, Assistant Chairman, and C. E. Falk as Administrative Scientist. This Department is responsible for the development, design and construction of the new 25 -Bev alternating gradient synchrotron. In addition, it is responsible for general research and development in the field of accelerator improvements. The Atomic Energy Commission and Associated Universities have agreed that the Laboratory will handle all the contractual arrangements for the construction of the alternating gradient synchrotron. Major decisions are made through a Steering Committee for the project which has as its members L. J. Haworth, G. K. Green, J. P. Blewett, H. D. Synder, D. D. Jacobus, M. M. Small, and C. E. Falk as Secretary. Dr. Green functions as Project Engineer with J. P. Blewett and D. D. Jacobus as deputies. M. M. Small is Construction Manager and is responsible for relationships with the architect-engineer and other subcontractors and for general scheduling and budget control. This type of organization and operation brings to bear upon a major construction effort the abilities of several specialists, all of whom have had substantial experience in the design, construction, and operation of the Cosmotron and other large facilities. In keeping with the basic idea that the Mechanical Engineering Division of the Laboratory shall be attached to the division having the greatest demand upon its services, the Mechanical Engineering Division is being transferred from the Cosmotron Department to the Accelerator Development Department as of July 1,1954 .

A second significant organizational change to be effected early in the next fiscal year and reflected in the organizational chart is the addition of an assistant director whose responsibilities will cover the general areas of plant operations and procurement. Included under procurement is the receipt, handling, and distribution of all materials obtained by the Laboratory. Mr. William H. Fields, Jr., formerly associated with the Controls Systems Laboratory of the University of Illinois and prior to that with the Radiation Laboratory of the University of California, will join the Laboratory on July 1st as Assistant Director and will be responsible for the operations indicated above.

Mr. J. G. Peter will continue to have charge of the Architectural Planning Division and will serve on the Director's staff in the capacity of Architectural
Planning Director. These changes will be effected in order to provide more time and more help to the Director, the Deputy Director, and the Director's staff for over-all planning purposes both with regard to the research program and to the future of the site. The day-to-day service and administrative operations are to be handled by the new Assistant Director.

\section{FINANCE}

Three program divisions of the Atomic Energy Commission finance the research program of the Laboratory. These three divisions, the Reactor Development Division, the Research Division, the Biology and Medicine Division, each separately finance research activities. However, to carry out these activities it is necessary for the Laboratory to provide general operation and administration and support of the technical activities as well as the direct research in each activity. The operating costs of the Laboratory for the past three fiscal years are shown in Fig. ure 12. These costs have been distributed among the programs supporting the work and also by major categories of expenditure. It is interesting to note that $781 / 2$ percent of all expenditures in FY 1954 are accounted for by labor costs. Nonprogram costs include work done by Laboratory organizational units for the construction of plant and equipment and work done for others, such as other government agencies, special work for other Commission programs not included under the normal Laboratory budget.

Detailed expenditures by organizational units are shown in Figure 13. The year end total for FY 1954 is $\$ 9,956,630$ which includes work done for others $(\$ 46,664)$ and work done by organizational units for plant and equipment $(\$ 209,123)$. This latter item showed a marked increase for the report year because of the initiation of the AGS project. The direct costs of AGS design and development are not shown in Figure 13. It should be noted that the expenditures associated with the direct research, i.e. Physical Sciences, Life Sciences, Applied Research, are their direct costs only and do not include services and overhead. Cost of work carried out directly by the research units increased by more than $\$ 560,000$ over that for FY 1953 while the total expenditures of the Laboratory increased by only $\$ 366,000$. This difference of almost $\$ 200,000$ means that expansion 
ORGANIZATIONAL EXPENDITURES - FISCAL 1952, 1953, 1954

(Includes Operating, Services to Capital Equipment and Facilities, Work for Others)

\begin{tabular}{|c|c|c|c|c|c|c|c|c|c|c|c|c|}
\hline \multicolumn{13}{|c|}{$\begin{array}{l}\text { ORGANIZATIONAL EXPENDITURES - FISCAL 1952, 1953, } 1954 \\
\text { (Includes Operating, Services to Capital Equipment and Facilities, Work for Others) }\end{array}$} \\
\hline & & $\begin{array}{l}\text { Salaries } \\
\text { \& Wages }\end{array}$ & $\begin{array}{l}\text { Consultants } \\
\text { \& Temporary } \\
\text { Appointments }\end{array}$ & Insurance & Travel & $\begin{array}{l}\text { Materials } \\
\quad \& \\
\text { Supplies }\end{array}$ & $\begin{array}{l}\text { Research \& } \\
\text { Development } \\
\text { Subcontracts }\end{array}$ & $\begin{array}{l}\text { Special } \\
\text { Power }\end{array}$ & $\begin{array}{l}\text { Miscellaneous } \\
\text { (Net) }\end{array}$ & $\begin{array}{c}\text { Total } \\
\text { Organizational } \\
\text { Costs }\end{array}$ & $\begin{array}{c}\% \\
\text { of } \\
\text { Total }\end{array}$ & $\begin{array}{c}\text { Capital } \\
\text { Equipment } \\
\text { (Total } \\
\text { Expense) }\end{array}$ \\
\hline \multirow[t]{3}{*}{ Physical Sciences } & 1954 & $1,347,272$ & 82,913 & 91,403 & 47,740 & 312,674 & 71,808 & 47,075 & $(42)$ & $2,000,843$ & 20.2 & 103.921 \\
\hline & 1953 & $1,144,834$ & 63,121 & 78,964 & 37,021 & 325,991 & 73,497 & 34,319 & (122) & $1,757,625$ & 18.4 & 155,363 \\
\hline & 1952 & $1,111,460$ & 52,389 & 73,191 & 48,752 & 268,419 & (12) & 22,655 & (243) & $1,576,611$ & 17.1 & 107,166 \\
\hline \multirow[t]{3}{*}{ Life Sciences } & 1954 & 858,666 & 38,373 & 57,836 & 30,344 & 204,575 & 68,516 & - & $(7,937)$ & $1,250,373$ & 12.6 & 26,819 \\
\hline & 1953 & 763,932 & 27,949 & 51,673 & 32,190 & 178,230 & - & - & $(8,802)$ & $1,045,172$ & 10.9 & 47,151 \\
\hline & 1952 & 702,077 & 15,713 & 45,417 & 26,888 & 211,359 & $=$ & - & $(8,483)$ & 992,971 & 10.8 & 42,301 \\
\hline \multirow[t]{3}{*}{ Applied Research } & 1954 & 860,759 & 25,576 & 56,833 & 31,320 & 299,181 & 42,735 & - & $(34,759)$ & $1,281,645$ & 12.9 & 125,738 \\
\hline & 1953 & 724,401 & 26,481 & 47,998 & 29,292 & 348,184 & 25,926 & - & $(12,869)$ & $1,189,413$ & 12.4 & 92,435 \\
\hline & 1952 & 540,047 & 21,472 & 33,931 & 24,542 & 304,225 & 24,970 & - & $(10,366)$ & 938,821 & 10.1 & 140,231 \\
\hline \multirow[t]{3}{*}{ Radiation Protection } & 1954 & 189,696 & 492 & 12,396 & 1,720 & 33,116 & - & 1,669 & $(11,422)$ & 227,667 & $2 . \overline{3}$ & 2,251 \\
\hline & 1953 & 208,735 & 1,844 & 13,663 & $\begin{array}{l}3,058 \\
3,05\end{array}$ & 50,758 & - & 2,145 & $(1,028)$ & 279,175 & 2.9 & 6,090 \\
\hline & 1952 & 223,659 & 2,244 & 14,344 & 5,362 & 26,665 & 18,300 & 3,478 & & 294,052 & 3.2 & 9,295 \\
\hline \multirow{3}{*}{$\begin{array}{l}\text { Supporting Scientific } \\
\text { \& Technical Services }\end{array}$} & 1954 & $1,368,601$ & 6,909 & 88,944 & 10,863 & 236,877 & - & 4423,545 & $(153,649)$ & $1,982,090$ & 19.8 & 63,215 \\
\hline & 1953 & $1,438,589$ & 4,448 & 93,845 & 6,003 & 221,085 & - & 439,881 & $(173,119)$ & $2,030,732$ & 21.2 & 102,443 \\
\hline & 1952 & $1,293,794$ & 2,608 & 82,496 & 5,915 & 201,288 & $=$ & 439,825 & $(53,914)$ & $1,972,012$ & 21.4 & 65,697 \\
\hline \multirow{3}{*}{$\begin{array}{l}\text { Security, Police and } \\
\text { Fire Protection }\end{array}$} & 1954 & 464,429 & - & 29,932 & 1,058 & 4,421 & - & - & - & 499,840 & 5.1 & - \\
\hline & 1953 & 504,537 & - & 32,690 & 52 & 6,358 & - & - & - & 543,637 & 5.7 & 457 \\
\hline & 1952 & 491,883 & - & 31,355 & 32 & 13,155 & - & $=$ & & 536,425 & 5.8 & 50 \\
\hline \multirow{3}{*}{$\begin{array}{l}\text { Miscellaneous } \\
\text { (net of income) }\end{array}$} & 1954 & - & - & - & - & 54,226 & - & - & 237,730 & 291,956 & 2.9 & 44,810 \\
\hline & 1953 & - & - & - & - & 32,513 & - & - & 202,277 & 234,790 & 2.4 & 59,973 \\
\hline & 1952 & - & - & & 32 & 17,681 & $=$ & - & 181,525 & 199,238 & 2.2 & 54,691 \\
\hline \multirow{3}{*}{$\begin{array}{l}\text { General \& } \\
\text { Administrative }\end{array}$} & 1954 & $1,798,659$ & 7,299 & 117,898 & 13,932 & 414,228 & - & - & $(116,610)$ & $2,235,406$ & 22.3 & \\
\hline & 1953 & $1,812,284$ & 2,507 & 118,638 & 12,856 & 475,645 & - & - & $(98,684)$ & $2,323,246$ & 24.3 & 3,421 \\
\hline & 1952 & $1,978,153$ & 672 & 125,957 & 14,426 & 444,940 & - & - & $(95,778)$ & $2,468,370$ & 26.7 & \\
\hline \multirow[t]{3}{*}{ Laboratory Total } & 1954 & $6,888,082$ & 161,562 & 455,242 & 136,977 & $1,559,298$ & 183,059 & 472,289 & $(86,689)$ & $9.769,820(1)$ & 98.1 & 386,589 \\
\hline & 1953 & $6,597,312$ & 126,350 & 437,471 & 120,472 & $1,638,764$ & 99,423 & 476,345 & $(92,347)$ & $9,403,790(2)$ & 98.2 & 467,333 \\
\hline & 1952 & $6,341,073$ & 95,098 & 406,691 & 125,949 & $1,487,732$ & 43,258 & 465,958 & 12,741 & $8,978,500(3)$ & 97.3 & 419,431 \\
\hline \multirow{3}{*}{$\begin{array}{l}\text { AUI Administration } \\
\text { and Public Education }\end{array}$} & 1954 & & - & - & - & - & - & - & 150,000 & 150,000 & 1.7 & - \\
\hline & 1953 & - & - & - & - & - & - & - & 150,000 & 150,000 & 1.6 & - \\
\hline & 1952 & 12,420 & & 792 & 1,047 & 3,402 & & & 152,218 & 169,879 & 1.8 & \\
\hline \multirow{3}{*}{$\begin{array}{l}\text { Total: } \\
\text { AUI and BNL }\end{array}$} & 1954 & $6,888,082$ & 161,562 & 455,242 & 136,977 & $1,559,298$ & 183,059 & 472,289 & 63,311 & $9,919,820(1)$ & 99.6 & 386,589 \\
\hline & 1953 & $6,597,312$ & 126,350 & 437,471 & 120,472 & $1,638,764$ & 99,423 & 476,345 & 57,653 & $9,553,790(2)$ & 99.8 & 467,333 \\
\hline & 1952 & $6,353,493$ & 95,098 & 407,483 & 126,996 & $1,491,134$ & 43,258 & 465,958 & 164,959 & $9,148,379(3)$ & 99.1 & 419,431 \\
\hline \multirow{3}{*}{$\begin{array}{l}\text { Work for Others, } \\
\text { Direct Costs Only }\end{array}$} & 1954 & 11,503 & 20 & 681 & 373 & 24,253 & - & - & - & 36,810 & .4 & - \\
\hline & 1953 & 7,677 & - & 560 & 181 & 1,958 & 4,078 & - & - & 14,454 & 0.2 & - \\
\hline & 1952 & 40,838 & 6,319 & 2,665 & 12,123 & 23,885 & & 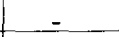 & & 85,830 & 0.9 & \\
\hline \multirow[t]{3}{*}{ Grand Total } & 1954 & $6,899,585$ & 161,562 & 455,923 & 137,350 & $1,583,551$ & 183,059 & 472,289 & 63,311 & $9,956,630(1)$ & 100.0 & 366,854 \\
\hline & 1953 & $6,604,989$ & 126,350 & 438,031 & 120,653 & $1,640,722$ & 103,501 & 476,345 & 57,653 & $9,568,244(2)$ & 100.0 & 467,333 \\
\hline & 1952 & $6,394,331$ & 101,417 & 410,148 & 139,119 & $1,515,019$ & 43,258 & 465,958 & 164,959 & $9,234,209(3)$ & 100.0 & 419,431 \\
\hline \multicolumn{13}{|c|}{$\begin{array}{l}\text { (1) } \$ 35,408 \text { of this total is also included in Capital Equipment Expense. } \\
\text { (2) } \$ 79,667 \text { of this total is also included in Capital Equipment Expense. } \\
\text { (3) } \$ 96,094 \text { of this total is also included in Capital Equipment Expense. }\end{array}$} \\
\hline
\end{tabular}




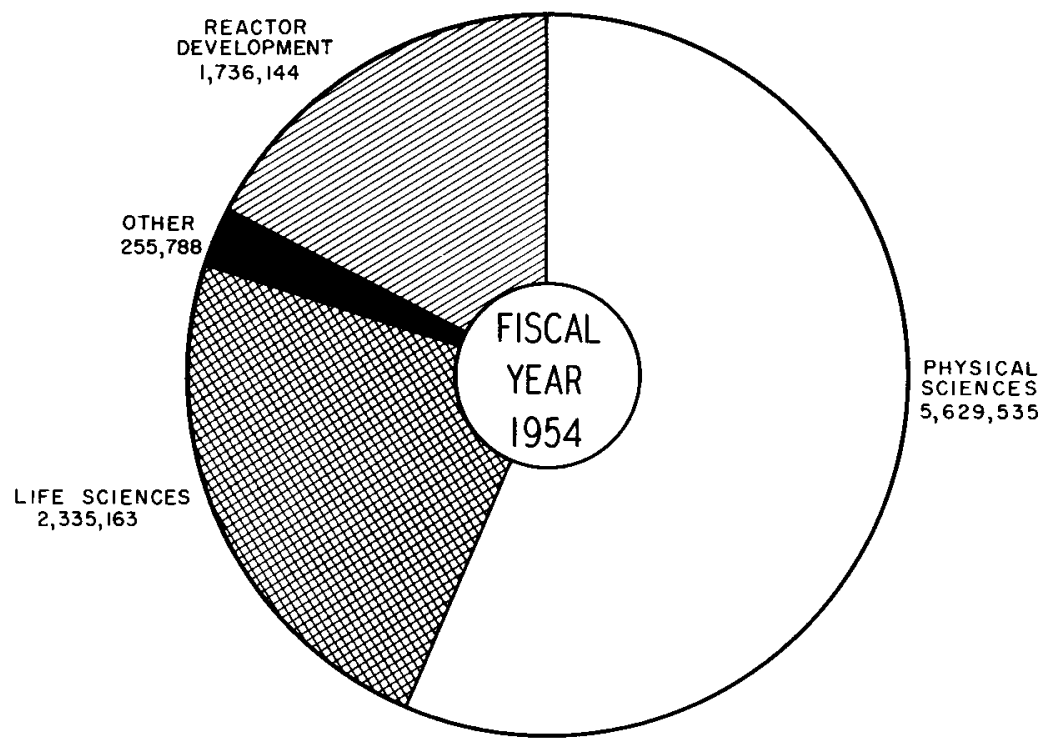

COST DISTRIBUTION BY PROGRAMS
\begin{tabular}{|c|c|c|r|r|c|}
\hline YEAR & $\begin{array}{c}\text { PHYSICAL } \\
\text { SCIENCES }\end{array}$ & $\begin{array}{c}\text { REACTOR } \\
\text { DEVELOPMENT }\end{array}$ & $\begin{array}{c}\text { LIFE } \\
\text { SCIENCES }\end{array}$ & OTHER & $\begin{array}{c}\text { TOTAL } \\
\text { COST }\end{array}$ \\
\hline 1952 & $57.8 \%$ & $12.3 \%$ & $24.0 \%$ & $5.9 \%$ & $9,234,209$ \\
\hline 1953 & $57.2 \%$ & $17.7 \%$ & $23.2 \%$ & $1.9 \%$ & $9,568,244$ \\
\hline 1954 & $56.5 \%$ & $17.4 \%$ & $23.5 \%$ & $2.6 \%$ & $9,956,630$ \\
\hline
\end{tabular}

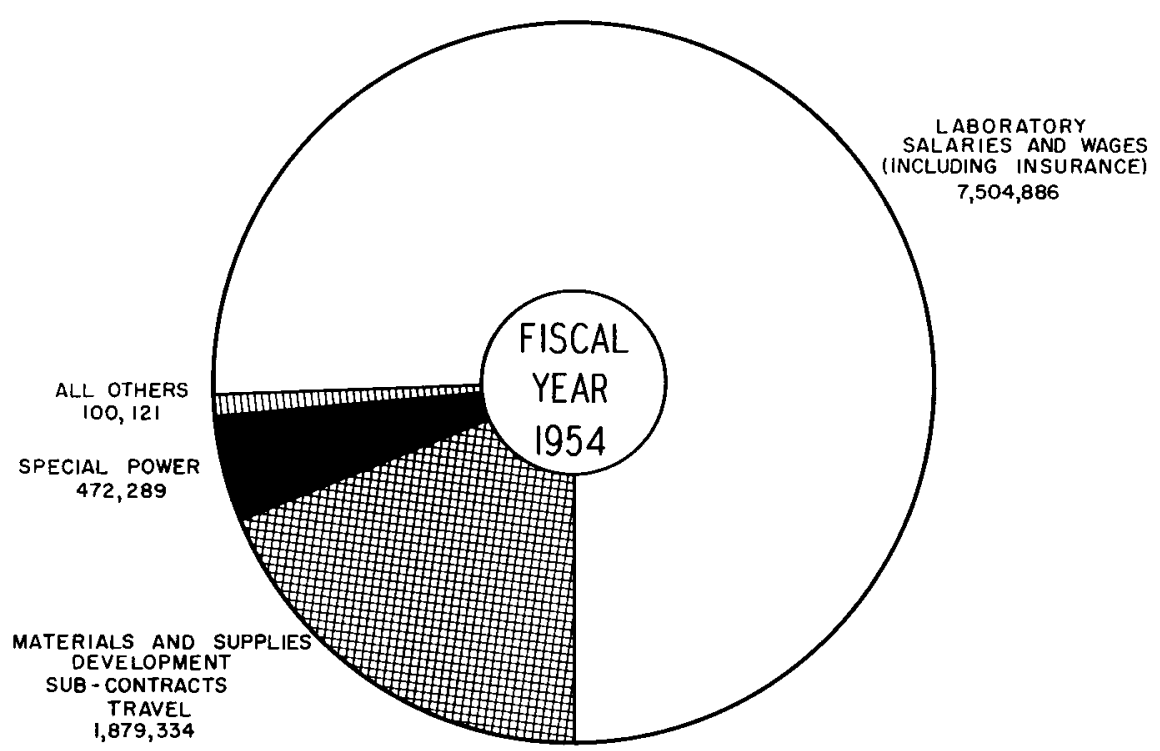

MAJOR CATEGORIES OF EXPENDITURES

\begin{tabular}{|c|c|c|c|c|c|}
\hline \multirow{2}{*}{ YEAR } & $\begin{array}{c}\text { LABORATORY } \\
\text { SALARIES AND WAGES }\end{array}$ & $\begin{array}{c}\text { MATERIALS } \\
\text { AND SUPPLIES }\end{array}$ & $\begin{array}{c}\text { SPECIAL } \\
\text { POWER }\end{array}$ & $\begin{array}{c}\text { ALL } \\
\text { OTHERS }\end{array}$ & $\begin{array}{c}\text { TOTAL } \\
\text { COST }\end{array}$ \\
\hline 1952 & $74.1 \%$ & $17.9 \%$ & $5.1 \%$ & $2.9 \%$ & $9,234,209$ \\
\hline 1953 & $74.9 \%$ & $19.1 \%$ & $4.9 \%$ & $1.1 \%$ & $9,568,244$ \\
\hline 1954 & $75.4 \%$ & $18.9 \%$ & $4.7 \%$ & $1.0 \%$ & $9,956,630$ \\
\hline
\end{tabular}

Figure 13. 
of the research effort came about through over-all expansion and through a decrease in indirect administrative and technical services. Expressed in another way, the direct research effort measured by expenditures accounted for 46.1 percent of the total in FY 1954 and 41.9 percent in FY 1953. When one includes technical services, these figures become 68.2 percent and 66.0 percent, respectively. Part of the decrease in technical services in FY 1954 can be ascribed to a reduction in service, especially reactor operations, due to the enforced curtailment of reactor operating level as a result of budget exigencies. However, as a general observation, it can be said that experience in all units which provide routine service is permitting more efficient operation with the accompanying reduction in cost or increase in service.

The Laboratory Balance Sheet as of June 30, 1954 is shown on p. xxxi with June 30, 1953 figures given for comparison. The large change in fixed assets reflects the yearly depreciation of plant and equipment with relatively minor additions of new plant. The beginning of AGS construction is seen as an increase in construction in progress assets. The $\$ 462,962$ inventory includes $\$ 66,000$ of precious metals and radium in addition to the regular stores inventory. From an operating point of view the reduction of inventory is significant; it has been mentioned earlier.

\section{PERSONNEL}

The over-all personnel trends for the report year have been similar to those in the past in that there was a net increase in the scientific staff and in the technical supporting staff and a net decrease in the staff of the general service and administrative units. The change in the latter category was quite marked because of changes in the Fiscal and Security Divisions. The changes in fiscal procedures resulting from the introduction of punch card equipment reduced the personnel in that Division by 16 people. A review of physical security risks brought about a revision of the number of guard posts required. New procedures and revised post assignments resulted in a requirement for 17 fewer patrolmen. This latter reduction was effected in December 1953. For the Laboratory as a whole there was a net gain of 15 during the report year because of the inauguration of new activities such as the AGS project. As of June 30 , 1954, some 63 people were associated with the AGS project. These people were recruited from both existing Laboratory organizations and as new employees.

Significant over-all changes in the general and administrative service units are not contemplated for the future. It is recognized that whereas reductions may be made in some areas of work, additions are necessary in other phases which take on a greater importance with the changing program. For example, the personnel level of the Buildings and Grounds Division remained essentially constant throughout the entire year; however, while in fiscal 1953 this unit contributed almost 30 man-years of direct services to the research program, in FY 1954, this same unit contributed 37 man-years with no increase in total personnel. The net effect, of course, was a reduction in service to the general and administrative function to the Laboratory. This reduction was possible almost entirely through the improvement of heating plant facilities and the requirement of less labor.

The growth of the scientific staff from July 1949 to date can be seen in Figure 14. The actual number of man-months of work performed by the scientific staff is plotted for each month. The graph is therefore an indication of scientific services rendered and is also a measure of the average number of scientists present each month. The regular staff increased about 10 percent during the year. Most of this increase occurred in the Accelerator Development Division. Salaried visitors include scientists and engineers on leave from their own institutions, graduate students doing doctoral investigations while on leave from their graduate schools and temporary appointees whose services are in some measure requested by the Laboratory. Guests include scientists and engineers who participate in the Brookhaven program but receive no remuneration for their services. The services of consultants are not noted in the graph. However, during the fiscal year 1954 over 26 man-months of service were rendered by these consultants.

For the seventh successive year, the number of scientific visitors who worked at the Laboratory has increased. A significant fraction of these visitors who spend periods of from one month to several years away from their home institutions and participate in the program of the Laboratory come without salary compensation. Of a total of 364 man-years of scientific effort in the report period, some 91 man-years 


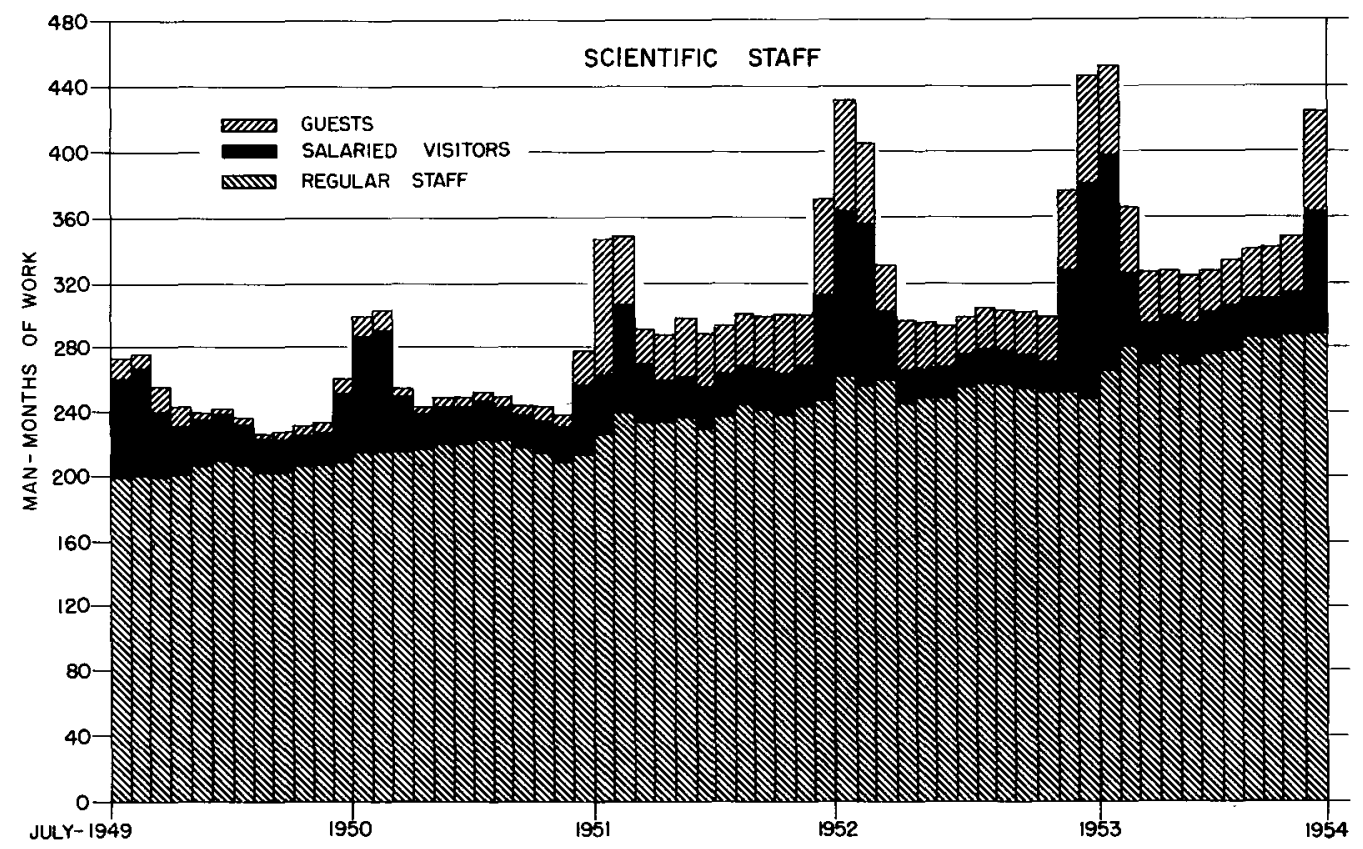

Figure 14 .

were contributed by salaried visitors, guests, temporary employees and consultants and of these 91 manyears, more than 40 were contributed by guests who received no remuneration from the Laboratory for their services. The continuing increase in number in the guest category is gratifying and reflects an expanding interest on the part of scientists to make use of the facilities available at the Laboratory.

The formal summer program of participation by graduate and undergraduate students, initiated in 1952, has been continued. The circulation of announcements for the summer of 1954 to universities and colleges resulted in applications from 354 students. Appointments have been offered to 98 of these applicants and acceptances received from 45 undergraduates and 43 graduate students. In addition to their participation in the Laboratory's activities, the students will be given tours of all of the laboratories and informal talks on many phases of the research in progress.

In addition to the above student participation, about 85 faculty members from universities are planning to work at the Laboratory during the summer of 1954. Including 16 AEC Radiological Physics Fellows and about 20 additional graduate students who will be brought here by visiting scientists to act as their assistants, the influx to the scientific staff will number at least 200 , the largest yet experienced for the summer months.

\section{CONFERENCES}

Extensive technical conferences in addition to regularly scheduled seminars and colloquia were held in support of the individual research programs. During the report year two major biology symposia were held. One in August 1953 on abnormal and pathological plant growth was attended by 96 scientists from 45 universities and other research institutions. The second one in June 1954 had as its subject the thyroid gland; it was attended by 82 scientists from 44 institutions. The Laboratory was host in May 1954 to the AEC-sponsored Third Information Meeting on Hot Laboratories which drew 156 scientists from other AEC installations and AEC contractors.

A classified symposium on the radiation effects in graphite was held in September 1953; it was attended by 27 solid state physicists and metallurgists.

At the request of the Third Naval District, Brookhaven was host at a Naval Reserve Nuclear Physics Seminar in June. Fifty-seven reservists attended two weeks of lectures and demonstrations.

In November 1953 the Director invited the presidents of 87 of the leading colleges and universities 
in the northeastern region to send one or two representatives from each of their institutions to attend a a two-day conference on the subject of university cooperation with Brookhaven. The response to this invitation was gratifying. One hundred and four representatives from 65 different institutions attended the meetings on December 10 and 11.

The first day of the conference was devoted to acquainting the delegates with the research programs and facilities at Brookhaven. On the second day, after a survey of the various methods that had been developed to provide for university-BNL cooperation, the delegates divided into four groups: the physical sciences, engineering, biology, and medicine. These groups held separate meetings under the chairmanship of one of the delegates in each group. At these meetings, suggestions were crystallized for modification of the existing means of cooperation and for any new ways in which greater participation by university scientists and students might be encouraged. A general meeting of the delegates was then held with key members of the Brookhaven staff at which the chairman of each of the groups presented its recommendations. These recommendations are summarized as follows:

(1) That the Laboratory give more attention to the dissemination of information concerning the opportunities available at Brookhaven through wider distribution of sections of the Annual Report, news. letters, and announcements on the extent to which Brookhaven can support personnel exchange and on the living conditions on and around the Brookhaven site and in nearby communities.

(2) That courses primarily for faculty members be offered at Brookhaven in Radioisotope Techniques, Reactor Science and Engineering.

(3) That the Laboratory consider the endorsement of certain university proposals soliciting financial aid for collaborative work with Brookhaven from such agencies as the Atomic Energy Commission, National Science Foundation and other research foundations.

(4) That Brookhaven explore the possibility of participation in the cooperative field programs already started in undergraduate curricula at many institutions.

(5) That there was hearty endorsement of the summer program both for students and for faculty with the recommendation that these programs be expanded to the extent that Laboratory facilities would permit.

It is difficult to be certain whether the recent increase in participation by university staff members can be attributed to the conference on university cooperation or to the spreading of the story of Brookhaven by those who have participated in its program. In either case, the increased participation has been most gratifying.

The interest of technical societies, educational and civic groups in visiting the Laboratory has continued. During the past fiscal year 16 professional groups visited the Laboratory; three of these groups were paying repeat visits to seek additional information on particular phases of the Laboratory's research. Thirty-six groups, totaling approximately 1400 individuals, were guided through the Laboratory's facilities which are not under security restrictions.

Because a large number of requests for visits have been received from student groups and organizations including high school and undergraduate and graduate groups, the Laboratory has decided to institute a special "Students' Day." This will serve a double purpose. First, it will provide a better opportunity to show the students the Laboratory installation with particular attention to the field of science of special interest. Second, it will avoid the time-consuming individual visits by such groups which in the past have taken place often on Saturdays and holidays. The first "Students' Day" is planned for October 15, 1954 to precede the regular Laboratory "Visitors' Day" which is to be held on October 16 for the general public.

The spreading of interest in the Laboratory's activities to wider and wider areas is proved by the fact that requests for Laboratory speakers have been received not only from organizations which are located in the vicinity but also from groups as distant as Rhode Island, Connecticut, Massachusetts, and New Jersey. The Laboratory complied with these distant requests only when they came from technical organizations and when they could be handled without interfering unduly with the speaker's research schedule. Other requests were referred to either the NYOO of the AEC or another suitable agency in the particular neighborhood. Twenty-four professional and 33 civic organizations totaling an audience of over 5000 individuals were addressed by Laboratory speakers in the past fiscal year. 


\section{BROOKHAVEN NATIONAL LABORATORY}

BALANCE SHEET

June 30, $1954 \quad$ June 30, 1953

ASSETS

\author{
Cash \\ Accounts Receivable \\ Inventories \\ Advances, Deposits and Prepaid Items \\ Fixed Assets (less reserves of $\$ 17,158,648$ ) \\ Construction in Progress
}

Total Assets

LIABILITIES

Accounts Payable

Accrued Payroll

Atomic Energy Commission

Total Liabilities
$\$ \quad 469,933$
52,329 462,962
41,095
$39,910,013$
668,198

$\$ 41,604,530$

$\$ 43,292,839$

$\$ \quad 375,280$

36,552

604,119

40,139

$42,010,233$

226,516
108,781

$41,149,547$

$\$ 41,604,530$

$\$ \quad 335,243$

94,101

$42,863,495$

$\$ 43,292,839$ 


\section{PHYSICAL SCIENCES AND ENGINEERING}

\section{PHYSICS}

Research in physics, during the past year, has approached stabilization, and at the same time emphasis has shifted. More results are now being obtained with the existing facilities and apparatus and less construction of new equipment has been required. Full use is being made of the nuclear reactor and of all the accelerators ranging from the Cosmotron to the 18-inch cyclotron. The actual operation of the two cyclotrons and the 3-Mev electrostatic accelerator is carried out by the Physics Department while the Cosmotron is operated by the Cosmotron Department. Each department carries out research with its respective machines as well as operating them for others. The development of new accelerators and specifically the $25-\mathrm{Bev}$ alternating gradient synchrotron is being done by the Accelerator Development Department. The work on accelerator development and operations of the accelerators is reported in the Accelerator section immediately following while the reactor operations report will be covered in the Technical Services section.

With the steady operation of all the Brookhaven facilities, cooperative work with various outside institutions has increased in all fields. Often a research program is divided between Brookhaven and other institutions. In some cases, the work is done by guest scientists temporarily assigned to work at Brookhaven. The extent to which these cooperative programs of research have been successful can be illustrated by the tremendous growth in the high energy physics field during the past year. The earlier spectacular observations made in this field have now been displaced by research involving detailed quantitative measurements leading to further progress in an understanding of the nucleus and nuclear forces. This trend was also accompanied, by necessity, with increased scientific effort both by Brookhaven staff and by guest scientists and in actual Cosmotron operating time. The tremendous number of experiments now under way and the variety of conditions under which they are carried out at the Cosmotron is given in Table 1. The listing is arranged by the type of re- search equipment being used. The extensive cooperative nature of the work can be seen from the listing of the investigator's home institution. It is to be noted that there are in use, or soon will be ready for use, six cloud chambers of various designs, two being operated by the Brookhaven staff while Yale, Harvard, Columbia and MIT operate the remaining four. Eleven institutions from many sections of the country are being supplied with photographic emulsions which, with the assistance of the Laboratory staff, are exposed to various high energy beams from the Cosmotron. Over 100 scientists are using the Cosmotron in their research. This number includes outside research collaborators as well as members of the Cosmotron, Physics, and Chemistry Departments. Approximately 50 of these are associated with outside institutions; 15 are radiochemists by profession. The use of the Cosmotron by the radiochemists (not indicated in Table 1) is described in the Chemistry section of this report.

Research in nuclear structure has produced many new results by the use of existing techniques refined and adapted in each case to the particular problem under investigation. Important progress has been made in the interpretation of decay schemes and excited levels of several nuclei and in the measurements of very short-lived isomers.

For the first time the spin of an isomer, namely calcium-134, was measured directly. The result was confirmed within a few hours by studying the decay scheme. The high intensity of the slow neutron flux from the Brookhaven reactor made possible the high precision determination of the neutron's magnetic moment. The method used was heretofore only possible with atomic beams. The experiment was performed in collaboration with physicists from Harvard University.

Neutron physics research has continued to progress smoothly and the equipment is also being improved steadily and data are now sufficiently detailed to allow comparison with theory. Brookhaven is continuing to fulfill its obligation in the compilation of neutron cross sections data for use in the atomic energy program and elsewhere. 
Table 1

Experiments in Progress

\section{A. Cloud Chambers}

\begin{tabular}{|c|c|c|c|c|}
\hline & Experiment & Incident beam used & Cloud chamber used & Institution of investigators \\
\hline 1. & $\pi-p$ scattering & $0.260 .35 \mathrm{Bev} \pi^{+}$ & BNL & Cornell $+\mathrm{BNL}$ \\
\hline 2. & $\pi-p$ scattering & $1.0 \mathrm{Bev} \pi^{-}$ & BNL & Rochester \\
\hline 3. & $\pi-\mathrm{d}$ scattering & $1.4 \mathrm{Bev} \pi$ & $\mathrm{BNL}$ & Fordham + BNL \\
\hline 4. & $\pi-\mathrm{N}$ scattering & 1.5 and $1.9 \mathrm{Bev} \pi^{-}$ & Harvard & Harvard \\
\hline 5. & $\mathrm{p}-\mathrm{p}$ scattering & $0.5-1.0 \mathrm{Bev}$ & BNL & Yale \\
\hline 6. & $\mathrm{n}-\mathrm{p}$ scattering & $\leq 1.4 \mathrm{Bev}$ & BNL & Purdue+BNL \\
\hline 7. & $\begin{array}{l}\pi-p \text { production and properties of } \\
\text { heavy unstable particles (H.U.P.) }\end{array}$ & $\overline{1.0} \mathrm{Bev} \pi^{-}$ & $\mathrm{BNL}$ & Rochester \\
\hline 8. & $\pi-d ;$ H.U.P. & $1.4 \mathrm{Bev} \pi^{-}$ & BNL & Fordham + BNL \\
\hline 9. & $\pi-\mathrm{N}^{2} ;$ H.U.P. & 1.5 and $1.9 \mathrm{Bev} \pi^{-}$ & Harvard & Harvard \\
\hline 10. & $\pi$-Fe; H.U.P. & $2 \operatorname{Bev} \pi$ & MIT & MIT \\
\hline 11. & $\pi$-Brass, Ph; H.U.P. & $2 \mathrm{Bev} \pi^{-}$ & Columbia & Columbia \\
\hline 12. & $\mathrm{P}-\mathrm{Be}, \mathrm{C}, \mathrm{Pb} ; \mathrm{H} . \mathrm{U} . \mathrm{P}$. & $3.0 \mathrm{Bev} \mathrm{P}$ & Yale & Yale \\
\hline 13. & n-p; H.U.P. & $\leq 1.4 \mathrm{Bev} \mathrm{n}$ & $\mathrm{BNL}$ & Purdue + BNL \\
\hline
\end{tabular}

B. Counters

\begin{tabular}{|c|c|c|}
\hline Experiment & Incident beam used & Institution of investigators \\
\hline 14. $\pi-p$ total cross section & $0.15 \cdots 0.75 \mathrm{Bev} \pi^{+}$and $\pi^{-}$ & BNL \\
\hline 15. $\pi-p$ diff. cross section & $0.25-0.35 \mathrm{Bev} \pi^{-}$ & BNL \\
\hline 16. $\pi-p$ total cross section & $0.5-2 \mathrm{Bev} \pi^{+}$and $\pi^{-}$ & Johns Hopkins + BNL \\
\hline 17. $\mathrm{p}-\mathrm{p}$ diff. scattering $\left(\mathrm{p}+\mathrm{p} \rightarrow \mathrm{d}+\pi^{+}\right)$ & $0.4-3 \mathrm{Bev} p$ & BNL \\
\hline 18. $\mathrm{p}-\mathrm{p}$ and $\mathrm{p}$-Be scattering & $0.5-3$ Bev p & BNL \\
\hline 19. $\mathrm{p}-\mathrm{p}$ and $\mathrm{p}-\mathrm{Be}$ production of $\pi^{\circ}, \gamma$ & $0.5-3 \operatorname{Bev} p$ & Cornell $+\mathrm{BNL}$ \\
\hline 20. $\mathrm{p}-\mathrm{p}$ and $\mathrm{p}-$ nuclei total cross section & $0.4-3 \mathrm{Bev} p$ & Harvard + BNL \\
\hline 21. p-target; $\gamma$ rays from H.U.P. & $\leq 3 \mathrm{Bev} p$ & BNL \\
\hline
\end{tabular}

C. Emulsions

\begin{tabular}{|c|c|c|}
\hline Experiment & Incident beam used & Institution of investigators \\
\hline 22. $\pi$-p scattering & $0.25 \mathrm{Bev} \pi^{+}$ & Carnegie Tech \\
\hline 23. $\pi-p$ scattering & $2 \mathrm{Bev} \pi^{-}$ & Chicago \\
\hline 24. $\pi-\mathrm{p}$ scattering & $2 \operatorname{Bev} \pi^{-}$ & Rochester \\
\hline 25. $\pi-p$ scattering & $2 \mathrm{Bev} \pi^{-}$ & Chicago \\
\hline 26. $\mathrm{p}-\mathrm{p}$ diff. scattering & 3 Bevp & Chicago \\
\hline 27. $\pi$-emulsion; heavy fragments & $1.25-2 \mathrm{Bev} \pi^{-}$ & Sarah Lawrence \\
\hline 28. p-target; H.U.P. & 2.2 and 3 Bev $p$ & Columbia \\
\hline 29. $\mathrm{p}$-target; H.U.P. & 2.2 and 3 Bevp & Illınois, MIT + BNL \\
\hline 30. $\mathrm{p}$-target; $\mathrm{K}^{-}$meson production & 3 Bevp & BNL \\
\hline 31. P-emulsion; H.U.P. & $3 \operatorname{Bev} p$ & Wisconsin \\
\hline
\end{tabular}

The solid state group continued the structural studies of solids, primarily in collaboration with Pennsylvania State University. The work on radiation effects was participated in by physicists assigned to Brookhaven from Watertown, Frankfort, and Picatinny Arsenals.

\section{HIGH ENERGY PHYSICS}

The research program in high energy physics is centered completely around the use of particles and radiations from the $3.0-\mathrm{Bev}$ proton synchrotron-the Cosmotron. The Cosmotron's usefulness lies in the variety of high energy beams it produces for experiments. The internal circulating beam now consists of pulses containing up to $5 \times 10^{10}$ highly monoenergetic protons. The particles are available at all energies from 0.1 to $3.0 \mathrm{Bev}$ and the energies are known to an accuracy of one percent. An external proton beam up to full energy can also be obtained with intensities up to a few $100 / \mathrm{cm}^{2}$ per pulse outside the radiation shield. In addition, several $\pi$-meson beams ranging in 
energy from $0.2 \mathrm{Bev}$ to $2.0 \mathrm{Bev}$ are now available outside the radiation shield. While the intensities of these beams, which are of the order of $5 / \mathrm{cm}^{2}$ per pulse, are not so high as one would like, they do permit many experiments with cloud chambers, counters and emulsions. Finally, high energy gamma rays and neutrons are also produced at the target in sufficient intensities to permit measurements concerning their interactions with matter as well as being, in themselves, an interesting subject for investigation.

These existing beams of protons, mesons, and neutrons are extremely useful for experiments because of their high energy and, in comparison with cosmic rays, their high intensity, and because of the large degree of control which can be exercised over them. The energy, position, intensity, time, and colli- mation of the beams are known and controlled, and the beams are available in a laboratory where large and intricate auxiliary apparatus may be set up to study the interactions.

It has now been shown that protons from the Cosmotron can produce all well-established varieties of heavy mesons and hyperons. While the number of these heavy particles which has been observed is not so large that it can be said that beams of particles have been established, an appreciable number of events has been observed.

\section{Research with Counters}

Since the Cosmotron provides nuclear particles with very much higher energies than previous accelerators, it is obviously important to explore this new

\section{STABLE AND UNSTABLE PARTICLES}

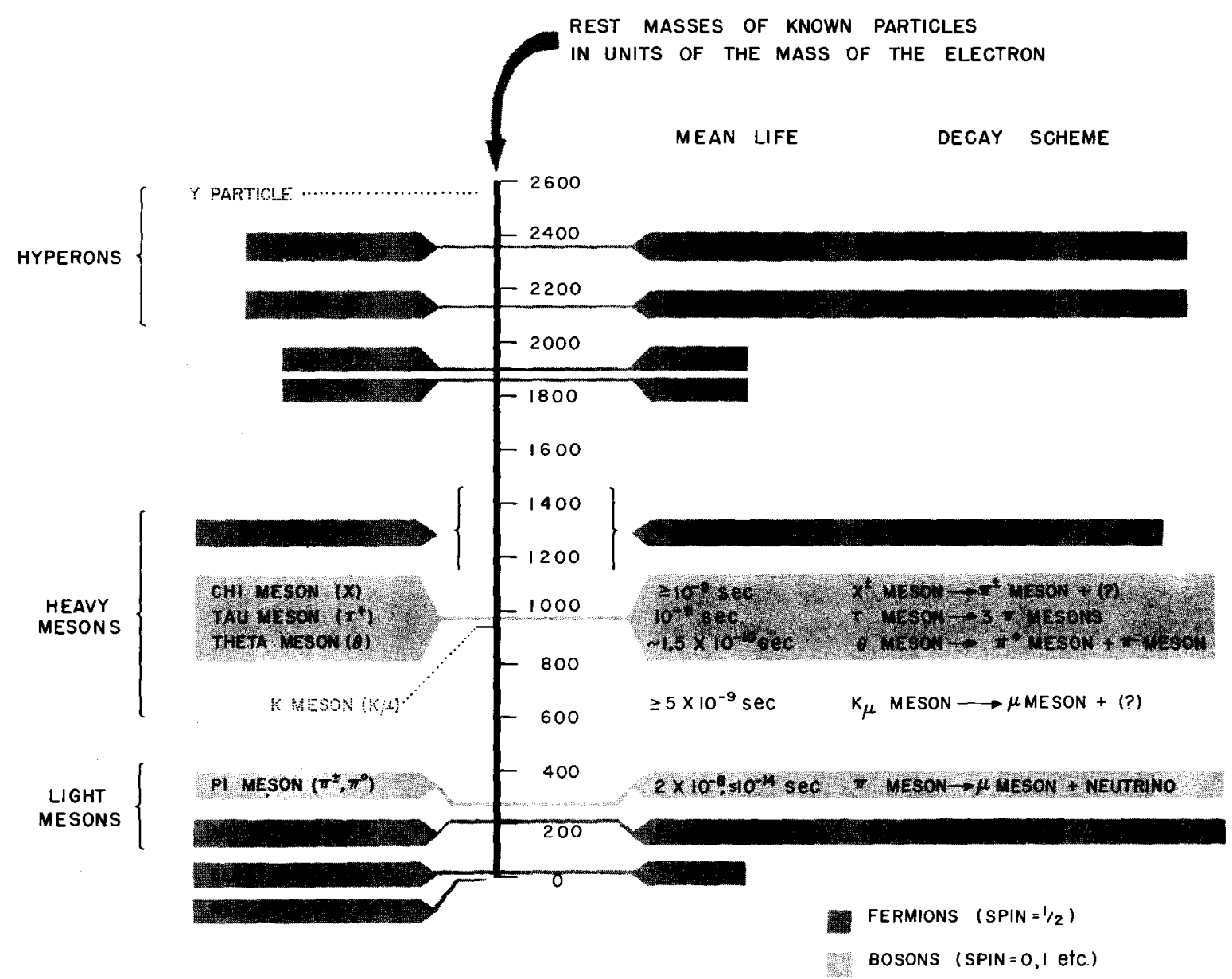

Figure 1. This chart gives the mass, mean life and mode of decay of all "elementary particles" whose existence is fairly well established. The unstable ones were all discovered in cosmic rays and the information presented here is not the result of work at this Laboratory. The Cosmotron, however, can produce all the mesons and hyperons shown and should prove effective in contributing to a better understanding of the nature and relationship of these fundamental particles. 
range of energies for new phenomena by extending types of measurements made with lower energy accelerators. The measurement of total scattering cross sections provides a means for searching a new region for unknown phenomena and has the advantage of requiring only modest intensities. Accordingly, most of the counter experiments performed so far have been measurements of this type. The results can be summarized in four curves showing $\mathrm{p}-\mathrm{p}, \mathrm{n}-\mathrm{p}, \pi^{+}$-p, $\pi^{-}-p$ total cross sections for incident particles having energies from $0.2 \mathrm{Bev}$ to $3 \mathrm{Bev}$ in the laboratory system. These curves are shown in Figures 2 and 3. The notable features of these curves are the rise in the p,p cross section between 400 and $800 \mathrm{Mev}$, which is not duplicated in the case of the $n, p$ total cross sections, the peak in the $\pi^{-}, p$ cross section around $1.0 \mathrm{Bev}$ and the $\pi^{+}, \mathrm{p}$ and $\pi^{-}, \mathrm{p}$ cross section peaks at $0.20 \mathrm{Bev}$. The rise in the $\pi, p$ cross sections at $0.20 \mathrm{Bev}$ was found by a University of Chicago group. The work at this Laboratory established that these cross sections fall off above $0.20 \mathrm{Bev}$. Since the positive pion beams have been obtained from the Cosmotron only up to about $1 \mathrm{Bev}$, the positive pion proton cross sections above 1 Bev have been deduced indirectly from measurements of the heavy waterwater difference. The accuracy depends on the validity of the charge symmetry hypothesis and of the assumption that the deuteron cross section should be approximately the sum of the free proton and neutron cross sections at these high energies. Measurements at $800 \mathrm{Mev}$, where both positive and negative pions are available, confirm the symmetry hypothesis, within the experimental error, and show that the deuteron cross section is smaller than the sum of the free nucleon cross sections by only approximately 10 percent.

There is a considerable body of theory bearing upon the $\pi, p$ peaks at $0.20 \mathrm{Bev}$ which explains these phenomena as the result of the formation of a very short-lived excited state of the nucleon. The existence of such of state should have an important if not dominant effect on more complicated interactions such as $\mathrm{p}, \mathrm{p}$ and $\mathrm{p}, \mathrm{n}$ interactions. The rise in the $\mathrm{p}, \mathrm{p}$ cross section corresponds to an available energy about equal to the energy of the excited state of the nucleon, which suggests that the effects due to the production of this state dominate the interaction. The absence of so strong a rise in the n,p cross section is consistent with the charge dependence expected from the excited state. Counter ex-

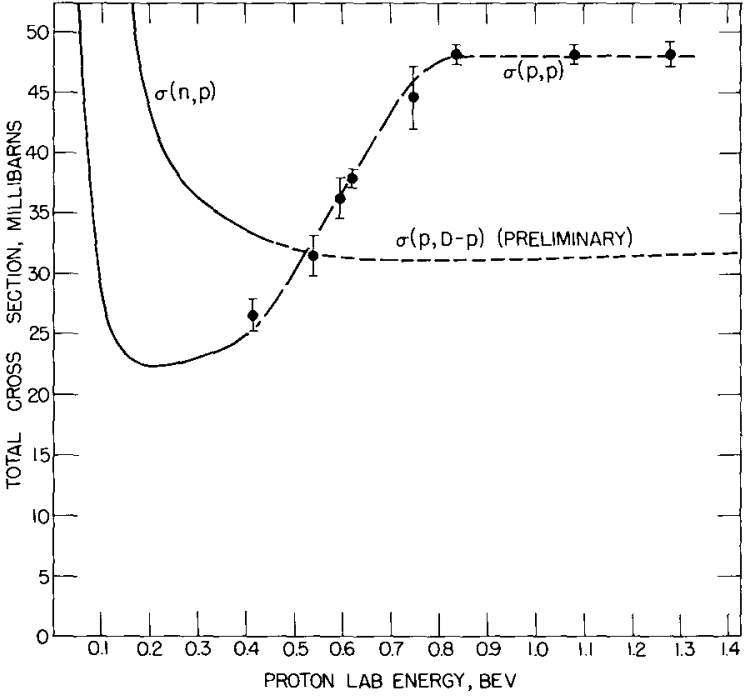

Figure 2. Total cross sections are a measure of the effective size of the colliding particles. These curves reveal an increase in the effective size of two colliding protons at laboratory energies between 0.4 and $0.8 \mathrm{Bev}$ and the absence of such an increase in the case of a proton colliding with a neutron. (Dashed curves show portion of curves obtained at Cosmotron.)

periments on the production of $\pi$-mesons by 1.0 - and 2.3-Bev protons striking beryllium and hydrogen support the view that the interaction occurs largely through the formation of this excited state.

The ratio of $\pi^{+}$- to $\pi^{-}$-mesons has been measured as well as the energy spectrum of the created $\pi$-mesons. If one assumes that the production of a single $\pi$-meson proceeds through the excited state of one of the nucleons which then decays by emitting a $\pi$ meson one can explain in qualitative manner major features of both the scattering and production processes. One can further deduce that single meson production predominates at $1.0 \mathrm{Bev}$ and double meson production at $2.3 \mathrm{Bev}$ as a consequence of exciting either one or both of the colliding nuclei. This concept that nucleons have excited states which decay with the emission of mesons gains support from detailed analysis of $\mathrm{n}, \mathrm{p}$ and $\mathrm{p}, \mathrm{p}$ collisions as observed in cloud chambers. If sufficient energy is available ( $E$ nucleon $>0.5$ Bev in center-of-mass system), n,p collisions result mostly in the production of a $\pi^{-}$-and a $\pi^{-}$-meson while p,p collisions mainly yield two $\pi^{+}$-mesons. The signs of the charged mesons produced are consistent with the idea that the excited neutron usually decays with the emission of a $\pi^{-}$-meson while the excited proton decays generally with the emission of $a \pi^{+}$-meson. The situation may 


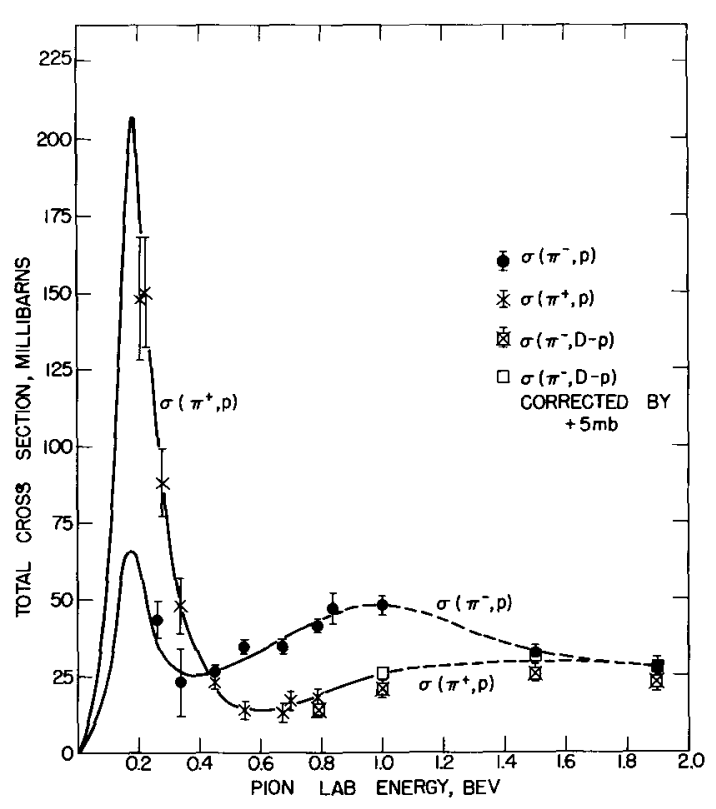

Figure 3. These curves show the results of $\pi$-meson scattering experiments carried only for the past year. Significant features revealed by this work are the rapid drop in the $\pi^{+}-$proton cross section above $0.2 \mathrm{Bev}$ and the maximum in the $\pi^{-}-$proton cross section at about 1.0 Bev.

well be complicated by other excited states of the nucleon and the simple picture described is surely incomplete. It appears clear, however, that the simple statistical predictions of meson production previously used to explain multiple production are not borne out.

A counter experiment has been performed to measure absorption and total neutron cross sections in the Bev range by a detector with a proton recoil device. The neutron beam from a Be target in the Cosmotron is peaked sharply forward. The detected neutron spectrum ranges from $0.4 \mathrm{Bev}$ to the maximum of over $2 \mathrm{Bev}$, but it is likely that the contribution below 1 Bev is small. The effective average energy is about 1.4 Bev. The attenuation difference observed between $\mathrm{CH}_{2}$ and $\mathrm{C}$ gave a total cross section for $\mathrm{H}$. The difference between $\mathrm{D}_{2} \mathrm{O}$ and $\mathrm{H}_{2} \mathrm{O}$ gave the neutron cross section; both $\sigma_{\mathrm{H}}$ and $\sigma_{\mathrm{N}}$ came out to be $42 \mathrm{mb}$, within the experimental error of about 5 percent. The absorption cross sections of eight elements, $\mathrm{Be}, \mathrm{C}, \mathrm{Al}, \mathrm{Cu}, \mathrm{Sm}, \mathrm{Pb}, \mathrm{Bi}$, and $\mathrm{U}$ were measured; the cross sections are well represented by assuming radii $\mathrm{R}=1.28 \mathrm{~A}^{1 / 3} \times 10^{-13} \mathrm{~cm}$, within an error of about 1 percent. The scattering cross sections for these nuclei agree with a change in wave number inside the nucleus of about $(0.15 \pm 0.005) \times 10^{13} \mathrm{~cm}^{-1}$.

\section{Research with Cloud Chambers}

Two diffusion cloud chambers have been exposed at the Cosmotron: one chamber is 16 in. in diameter, with a magnetic field of 10,000 gauss. The other chamber is $6 \mathrm{ft}$ long and 11 in. wide, without a magnetic field. Both chambers can be operated with hydrogen, deuterium, or helium at pressures up to 20 atmos. For most exposures, meson and nucleon beams were obtained from the Cosmotron by letting the circulating primary proton beam strike a carbon target with a subsequent magnetic analysis of the secondary particles so produced. A portion of the primary proton beam has also been obtained externally.

The main purpose of these studies is to investigate interactions occurring in the cloud chamber gas where unambiguous interpretation is often possible, particularly in the light gases used here. Mostly pions are produced in these interactions, but a number of events showing production of heavy unstable particles has also been observed. There have been 120,000 photographs obtained with the magnet chamber and 30,000 with the long chamber.

During recent months, the BNL group has been joined by members of several universities, especially in scanning and analyzing many of the photographs that are being obtained. Members from Cornell, Duke, Fordham, Purdue, Rochester, and Yale Universities and from the Naval Research Laboratory are presently involved in these efforts. Four thesis projects are among the problems under investigation. Several scanning and analyzing projectors have been constructed for use at some of the institutions mentioned. Much of the work so far constitutes only a qualitative survey of the different types of interactions to be expected at Cosmotron energies.

Cloud chambers have been set up at the Cosmotron by Yale, Harvard, and Columbia Universities and Massachusetts Institute of Technology is in process of setting up one. The Yale cloud chamber has been used to examine interactions of the high energy neutron beam with lead. Nine $\Lambda^{0}$ particles were identified and from this a cross section for production in lead of only $2 \mathrm{mb}$ was deduced. This result could be explained by a possible large absorption cross section for $\Lambda^{0}$ particles by the same lead nucleus in which they were produced. The Harvard cloud chamber has been operating in the 1.5-Bev $\pi^{-}$-meson beam and some 100,000 cloud chamber pictures have been obtained for detailed studies of the interaction of these mesons with the nuclei of nitrogen which fills the cloud 
chamber. The Columbia chamber is also operated in the 1.5 -Bev $\pi^{-}$-meson beam and this group is planning to take advantage of their large chamber and strong magnetic field to make accurate quantitative measurements on the decay products of heavy mesons. The MIT chamber probably has the largest sensitive volume of any in the world and contains some 17 half-inch separated iron plates in which heavy mesons can be produced by the incident particles from the Cosmotron; the energy range and scattering of heavy mesons will be studied by observing the tracks they leave between these plates.

The following paragraphs give brief descriptions of the principal researches performed with the BNL cloud chambers.

For neutron-proton collisions at neutron energies $\leq 2.2 \mathrm{Bev}$, the main result is that the reaction $\mathrm{n}+\mathrm{p} \rightarrow \mathrm{n}+\pi^{+}+\pi^{-}$is by far the most common. Single pion production is much less common, when compared with double pion production, than theoretical predictions had indicated. Triple pion production was not observed to occur. These facts, together with anisotropies found in the angular distributions of the emitted particles, indicate the possible existence of intermediate excited states of the nucleons after collision. The experiment is being repeated in a beam containing only neutrons with energies $\leq 1.4 \mathrm{Bev}$.

A study of reactions between negative pions and protons showed that for 1.4-Bev incident pions, about one-third of all collisions are elastic, with the pions mostly scattered quite forward, which suggests diffraction scattering. An interaction radius somewhat smaller than $1.4 \times 10^{-13} \mathrm{~cm}$ is indicated. Most of the inelastic collisions appear to result in single pion production, in agreement with Fermi's statistical theory. This is also indicated by the very small observed number of the especially easily recognized four-prong reactions $\pi^{-}+\mathrm{p} \rightarrow 2 \pi^{-}+\mathrm{p}+\pi^{+}$. Angular distributions of the collision products show that in the center-ofmass system the nucleons pass mostly backward. Additional events obtained with the long chamber are awaiting analysis. Photographs taken at $\pi^{-}$energies of 1.0 and $1.8 \mathrm{Bev}$ are being analyzed; those at 1.0 Bev are especially for the study of details of an energy maximum in the $\pi^{-}-\mathrm{p}$ total cross section observed near 1.0 Bev by counter methods. Photographs obtained with deuterium filling in cloud chamber with 1.4-Bev pions are being scanned to study $\left(\pi^{-}, \mathrm{n}\right)$ reactions. The results may be particularly sensitive to the existence of excited states of nucleons.
Analysis is proceeding on the study of protonproton collisions. For $1.5 \mathrm{-Bev}$ protons, a preliminary result is that the very small observed number of fourprong events from the reaction $\mathrm{p}+\mathrm{p} \rightarrow 2 \mathrm{p}+\pi^{+}+\pi^{-}$ may only be consistent with the large number of $\left(\pi^{+}, \pi^{-}\right)$pairs produced in neutron-proton collisions at similar energies, if the production of excited nucleon states is assumed. The experiment is being repeated with 2.9-Bev protons. Furthermore, an experiment with protons of much lower energies (0.4-0.9 Bev) is proceeding for a study of the rapid rise in the total $\mathrm{p}-\mathrm{p}$ cross section within this energy range, as observed by counter methods.

Total cross sections and angular distributions are being obtained for the scattering of positive pions on protons at energies between 200 and $400 \mathrm{Mev}$. A preliminary result was that near $230 \mathrm{Mev}$ the angular distribution of the scattered $\pi^{+}$becomes predominantly forward in the center-of-mass system while it has been observed that at somewhat lower energies the distributions are backward. It is hoped that the present studies will offer some aid in a selection of the proper set of scattering phase shifts from several theoretically possible sets.

Whenever the energy of the incident particles has been high enough, heavy unstable particles (V-particles) were observed decaying in the cloud chambers, and in some cases their origin of production could be located in the gas to permit rather detailed study. Ten cases of V-particle production have been found to date in collisions between 1.4 and $1.8 \mathrm{Bev}$ $\pi^{-}$-mesons with protons. In three of these cases, the decay of a charged $\mathrm{V}$ is observed. One of these is probably a negative hyperon. In one of the other seven cases, both a neutral hyperon and a neutral $\mathrm{K}$ particle are seen to decay some distance away from the point where a $1.4-\mathrm{Bev} \pi^{-}$collided with a proton in the cloud chamber gas. This event, together with an additional one where $\mathrm{V}$-production occurred in the cloud chamber wall, shows directly that V's can be produced doubly. In four of the remaining six cases, a neutral hyperon only is seen to decay and in the two other cases a neutral K-particle only. In all 10 cases the observed kinematical relations are not inconsistent with exclusive double production of $V$ 's. The angular distributions of the V's and their decay products are also investigated. Several departures from random distribution are indicated.

One case of V-production by a 2.9-Bev proton on a proton has so far been observed. The non-coplanar 
event is being studied. V-production events in nucleon-nucleon collisions are of particular interest to help in deciding the important theoretical question of whether or not hyperons can be produced in pairs. In connection with this question, it is of interest to state a qualitative result on the relative numbers of V's (neutral and charged) produced in the cloud chamber walls. The largest number of decays of such V-particles have been found in the 1.0, 1.4, and 1.8 Bev $\pi^{-}$beams. A few were found in the neutron beam of $\leq 2.2-\mathrm{Bev}$ energy. Several were observed in the 3-Bev proton beam, but none at all were found in the $1.5-\mathrm{Bev}$ proton beam. In the latter beam, the energy available for production of secondaries is the lowest and, including the Fermi energy of a nucleon in a nucleus, is only slightly above the threshold for production of a hyperon together with a K-particle, but farther above the threshold for production of two hyperons. The absence of V's in this beam thus indicates that pairs of hyperons may not be produced.

\section{Research with Nuclear Emulsions}

Photographic emulsion has been successfully used to reveal the existence of artificially produced heavy mesons and hyperons and to contribute to an understanding of their properties. The apparent simplicity of this technique for the study of high energy processes is deceptive and a great deal of explorative work was necessary before extraneous background could be eliminated and the unique characteristics of the Cosmotron's beams put to good advantage. The most fruitful beams have been 1) the full-energy proton beam directed to the emulsion itself at greatly reduced intensities; 2) the external 1.5 - to $2.0-\mathrm{Bev}$ $\pi^{-}$-meson beam analyzed by an electromagnet and directed through emulsions located outside the concrete shield; 3 ) the unanalyzed radiations emerging from an internal target bombarded with $3-\mathrm{Bev}$ protons with the emulsions located at one side a few inches distant.

The Brookhaven work falls essentially into two groups: studies of the interaction of pions (light, $\pi$-mesons) with nuclei, and of the production and properties of heavy $(\mathrm{K})$ mesons.

The interactions with emulsion nuclei of $0.5-\mathrm{Bev}$ negative pions, produced by the Cosmotron, were measured. The energy losses suffered by the pions in collisions with the nuclei, as well as their angular distribution as they were scattered in those collisions, were measured. In about 2 percent of the collisions, another charged light meson was produced, and six examples of the production of neutral pions were found. A rough estimate for the cross section for meson production, by mesons colliding with nucleons, was obtained (3-10 mb).

The emphasis in the work on heavy mesons was different because the existing knowledge of heavy mesons is considerably more elementary than that of light mesons. It is not known, from cosmic ray observations, how energetic protons must be to produce heavy mesons; it was not certain that heavy mesons could be produced at Cosmutron energies. Consequently, the first efforts were to find out whether $\mathrm{K}$. mesons were created here. The efforts were successful; both positive and negative $\mathrm{K}$-mesons created by collisions of 2.2- and 3-Bev protons with beryllium and with copper targets were observed in the emulsions. It was further established that these $\mathrm{K}$-mesons had masses of about 1000 electron masses. One of the principal questions about positive mesons is their mode of decay; do they decay into $\pi$ 's or the still lighter $\mu$-mesons, and when they decay, do they decay into two or three (or more) particles? It has been shown, for all those positive mesons created at the Cosmotron for which appropriate measurements have been completed, that they decay into $\mu$-mesons by three-body decay. In at least two cases of K-production, it was shown that the $\mathrm{K}$-mesons could not have been produced in pairs when the high energy proton collided with a nucleon of the target, an observation of interest to a hypothetical mechanism suggesting that they are produced in pairs.

A group at the University of Wisconsin has been studying emulsions exposed to the internal proton beam and has found some 14 examples of so-called unstable fragments. These are believed to be nuclear fragments in which a neutron has been replaced by a hyperon which in $10^{-10}$ to $10^{-12} \mathrm{sec}$ decays into charged particles which cause the fragment's track to end in a star.

A group at the University of Rochester has been examining emulsions exposed to the 2.0-, 1.5- and 1.0-Bev $\pi^{-}$-meson beams and have been able to identify over 50 interactions which have the characteristics of $\pi^{-}-\mathrm{p}$ collisions. Analysis of these events has revealed significant data on the production of $\pi^{ \pm}$and $\pi^{0}$ mesons, and the angular distribution of the resulting particles has been obtained. This group has also identified a number of unstable fragments and heavy mesons. 
Additional emulsion work is being done by physicists from the University of Chicago, Columbia University, the Massachusetts Institute of Technology, the University of Washington, and others.

\section{Theory}

Several recent experiments have shown that protons of energy 200-350 Mev scattered from nuclei are polarized. An improved calculation was made of the polarization to be expected when nucleons are elastically scattered from nuclei, assuming the existence of a spin-dependent nuclear potential similar to that assumed in the nuclear shell model. The WKB approximation was used to calculate the phase shifts from a nuclear complex potential, constant over a sphere, to which is added a term - $(1 / \hbar) U(r) \overline{\mathrm{L}} \cdot \overline{\boldsymbol{\sigma}}$ where $\bar{L}$ and $\hbar \bar{\sigma} / 2$ are the orbital and spin angular momenta of the proton. The polarization, $\mathrm{P}(\theta)$ was calculated for several choices of depth and shape of $\mathrm{U}(\mathrm{r})$. The experimentally observed value for 316 Mev of $\mathrm{P}(\theta)=0.60$ at $\theta=14^{\circ}$ can be easily fitted theoretically. A classification scheme of "new" unstable particles has been prepared.

\section{NUCLEAR STRUCTURE}

Nuclear spectroscopy has reached the stage where fairly complex decay schemes can be successfully

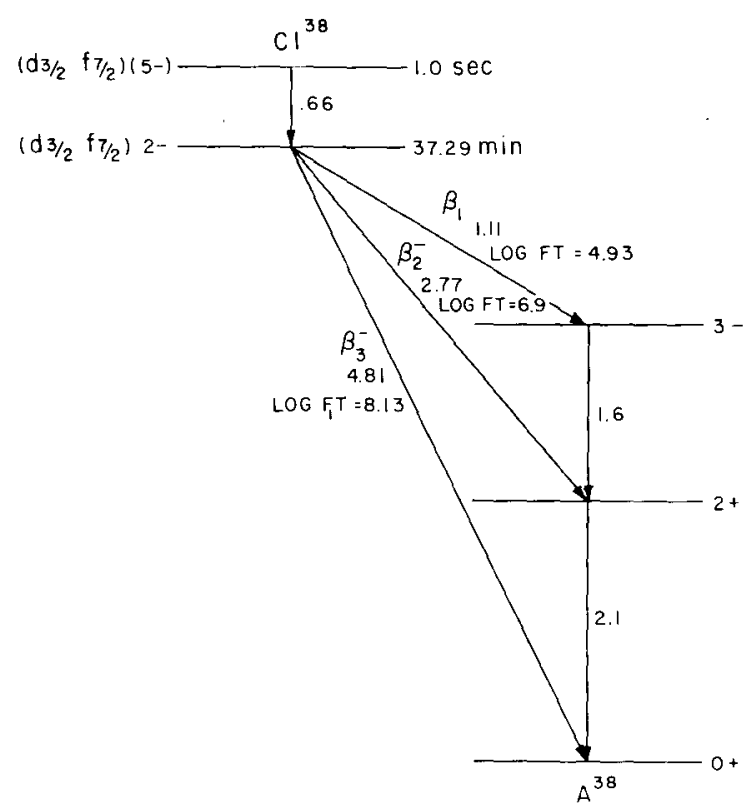

Figure 4. Decay scheme of $\mathrm{Cl}^{38 \mathrm{~m}}$ and $\mathrm{Cl}^{38}$. The new isomer was discovered by pile neutron irradiation of chlorine.

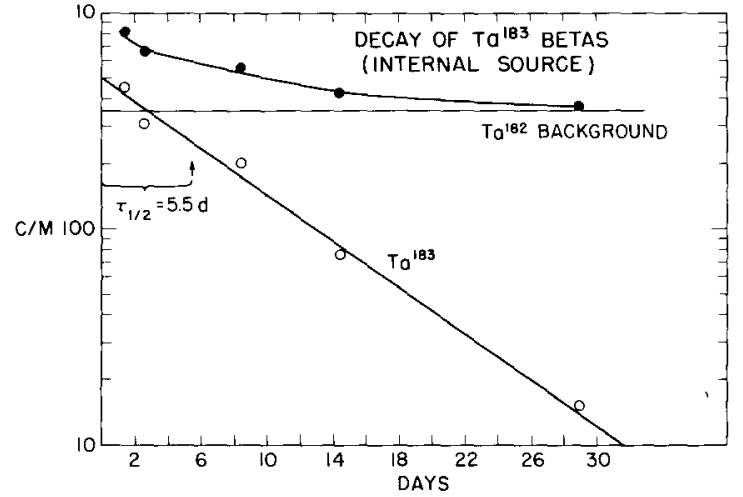

Figure 5. A NaI crystal was grown, which contained pileactivated Ta. Two activities are present, the result of two successive neutron captures:

$$
\mathrm{Ta}^{1 \times 1}+\mathrm{n} \rightarrow \mathrm{Ta}^{182}, \mathrm{Ta}^{1+2}+\mathrm{n} \rightarrow \mathrm{Ta}^{183} .
$$

interpreted. Many such schemes have been studied with improved techniques; high resolution magnetic spectrographs are utilized for electron detection and high speed coincidence techniques for nuclear lifetime determinations. Among the decay schemes recently studied are the following: $\mathrm{At}^{210}, \mathrm{Os}^{191}, \mathrm{Ta}^{1891}$, $\mathrm{Ta}^{1 \times 2}, \mathrm{~W}^{1 \times 4}, \mathrm{Hf}^{1 \times 1}, \mathrm{Hf}^{18011}, \mathrm{Ho}^{166 ;}, \mathrm{Sm}^{153}, \mathrm{Cs}^{131+1}, \mathrm{Cl}^{13 \times}$. and the decay of neutron-deficient $\mathrm{Hg}$ isotopes (and associated decay chains terminating at Pt or Ir) produced by $\mathrm{Au}(\mathrm{p}, \mathrm{xn}) \mathrm{Hg}$ reactions. Angular correlation measurements $(\gamma-\gamma)$ have been made in the decay of $\mathrm{At}^{210}, \mathrm{Hf}^{180 \mathrm{~m}}$, and $\mathrm{Cl}^{38}$. The case of $\mathrm{Hf}^{1 \mathrm{1num}}$ is particularly interesting in that the excited states afford almost perfect agreement with the predictions of the collective nuclear model of Bohr and Mottelson for the rotational spectrum of even-even nuclei. The angular correlation measurements confirm the rotational spin sequence $2,4,6,8$ for the first four excited states of $\mathrm{Hf}^{180 \mathrm{~m}}$, if a spin of zero is assured for the ground state.

New isomers formed by pile neutron irradiation of $\mathrm{Br}^{79}(210 \mathrm{kev} ; 5 \mathrm{sec})$ and $\mathrm{Cl}^{37}$ (660 kev; $\left.1 \mathrm{sec}\right)$ have been discovered (see Figure 4 ). The cross section for secondary neutron capture in $\mathrm{Ta}$ has been measured both by conventional and "internal source" techniques (see Figure 5). The lifetimes of a number of new short-lived isomers in the $10^{-9}$-sec range have been measured. These include the lifetimes of excited states in $\mathrm{Po}^{210}, \mathrm{~W}^{182}$. $\mathrm{Os}^{188}, \mathrm{Hf}^{180}, \mathrm{Gd}^{154}$ and $\mathrm{Sm}^{152}$. An interesting regularity of the matrix elements for fast E2 transitions in even-even nuclei has been discovered. Matrix elements for such transitions achieve a maximum value of $\sim 100$ (in single proton 
transition probability units) at about neutron number $\mathrm{N}=98$ and then decrease uniformly as $\mathrm{N}$ approaches magic number 126 (see Figure 6).

The mutual stabilization of proton and neutron orbits and the consequences of this stabilization on nuclear transition probabilities have been investigated theoretically and illustrated by many examples.

In collaboration with Oxford University and the Nobel Institute for Physics, Stockholm, a study of the energy level structure of $\mathrm{Pb}^{206}$ has been completed from which it has been possible to correlate in detail measurements of the energies, spins, and parities of 12 excited states with theoretical calculations based on the shell model. The lens-type beta-ray spectrometer has been used for a number of investigations of radioactive isotopes including $\mathrm{Bi}^{207}, \mathrm{I}^{132}$,

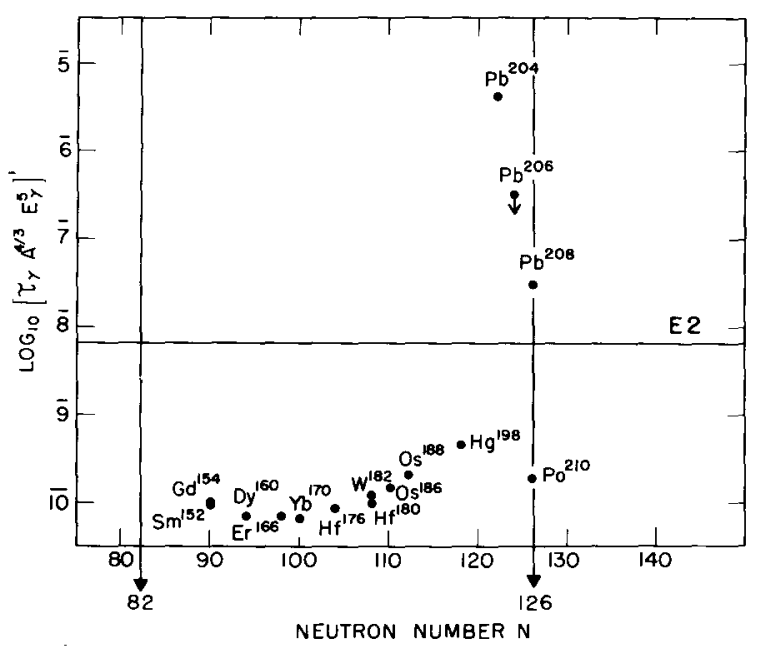

Figure 6. Matrix elements for E2 transitions as a function of neutron number.

$\mathrm{HF}^{11 \times 1}, \mathrm{~W}^{187}, \mathrm{As}^{210}, \mathrm{Ta}^{181}, \mathrm{Se}^{75}$, and $\mathrm{Ge}^{75} . \mathrm{A}$ threecrystal pair spectrometer has been developed and used successfully with a gray wedge pulse height analyzer to detect four new and very weak gamma rays emitted in the decay of $\mathbf{R h}^{106}$.

Design calculations have been made and construction has been started on a magnetic pair spectrometer based on a new principle for use in measuring high energy gamma rays associated with nuclear reactions produced by bombardment with the research electrostatic accelerator. The new instrument will be an iron-free intermediate-image beta spectrometer in which statistical separation detection of the pairs will be employed.

\section{ATOMIC AND MOLECULAR PHYSICS}

\section{Nuclear Spin of Metastable State of Cesium-134}

The atomic beam resonance apparatus is now in operation. With it the spin of the 3.1-hr isomeric state of $\mathrm{Cs}^{134}$ has been measured and found to have the unusually large value of $8 \mathrm{k}$.

The apparatus is of the atomic magnetic resonance type in which one observes a "flop-in" of the magnetic states which undergo the transition.

$$
[\mathrm{I}+1 / 2,-(\mathrm{I}+1 / 2)] \rightleftarrows[\mathrm{I}+1 / 2,-(\mathrm{I}-1 / 2)]
$$

In a weak magnetic field this transition frequency depends only upon I. In a higher magnetic field, this transition is dependent upon the hyperfine structure interaction $\Delta v$. By observing the transition for $\mathrm{Cs}^{134 \mathrm{~m}}$ up to $136 \mathrm{Mc}$ and calibrating the field with the corresponding transition for $\mathrm{Cs}^{133}$, it was possible to calculate the value of $\Delta v, 3675.6 \pm 0.6 \mathrm{Mc} / \mathrm{sec}$.

The beam detection system consisted of a tungsten target upon which the focused beam was allowed to impinge for an arbitrary time of $5-10 \mathrm{~min}$ for each observation. The target was then removed from the system via an airlock and the activity of the absorbed Cs measured with a proportional counter. The peak counting rates observed ran up to 70 cts/min with a background of about 15 .

From the value of $\Delta v$, it is possible to compute the nuclear magnetic moment by the GoudsmitFermi-Segré expression and the value $1.10 \pm .01$ nuclear magnetons is thus obtained. On the basis of shell theory, it appears that one must assume an admixture of $\left(g_{7 / 2}, h_{11 / 2}\right)$ and $\left(d_{5 / 2}, h_{11 / 2}\right)$ in order to explain the observed spin and magnetic moment.

\section{Magnetic Moment of the Neutron}

Physicists from Harvard University and BNL have determined the ratio of the magnetic moment of the neutron to that of the proton to a high degree of precision. A beam of neutrons emerging from the reactor impinges at a small glancing angle upon a polished cobalt mirror which is magnetized. The spins of the reflected neutrons are about 90 percent polarized with respect to the direction of the magnetic field. The reflected beam will then be highly reflected from a second such mirror. Between the mirrors, the neutrons pass through a region of uniform magnetic field where they may become depolarized by locally produced magnetic fields oscillating at the Larmor frequency. 
In order to achieve high precision, the path length in the field of a permanent magnet is $150 \mathrm{~cm}$. Two oscillating fields are used with a distance of about $110 \mathrm{~cm}$ between loops. This technique effectively averages the magnetic field over the path length and results in a resonance line about 360 cycles wide at about $25 \mathrm{Mc} / \mathrm{sec}$. The neutron resonance can be determined with an accuracy of considerably better than one part per million.

The proton resonance frequency is determined point by point along the trajectory. Because of the actual lack of uniformity of the field, both in space and in time, the precision of the average proton resonance frequency is uncertain by about two parts per million. At this date, the best value for the ratio of moments is $\mu_{\mathrm{n}} / \mu_{\mathrm{p}}=0.6850497 \pm 0.0000010$.

\section{Nuclear Magnetic Moment of Selenium-79}

In cooperation with physicists of Columbia University, and MIT, the Zeeman effect of the microwave spectrum of $\mathrm{Se}^{\text {ig }}$ was studied. This yielded a value of $-1.015 \pm 0.015$ nuclear magnetons for the magnetic moment of this nucleus.

\section{Nuclear Mass Measurements}

Work on the new radiofrequency mass spectrometer during the past year has centered on attempts to improve resolution and track down sources of systematic error. It has been possible at times to obtain a half-width resolution of one part in 31,000 at mass 28 and systematic errors have been reduced by redesign of the ion source and extensive shimming to make the magnetic field more uniform. However, further work along these lines is necessary to improve reliability of mass measurements. New water-cooled magnet coils have permitted use of higher magnetic fields and thus work at higher masses with reasonable ion energies. Preliminary measurements of the mass difference between the isotopes of xenon at masses 132 and 131 have yielded results in good agreement with recent measurements by more conventional methods. Here, half-width resolution of one part in 21,000 was obtained and direct comparison of isotopes of different mass number was done by "overlapping orders," a technique not possible in conventional instruments.

\section{Tritium Research}

By a new technique, the natural tritium concentration in a number of rains, well water, and Long
Island Sound water samples has been measured with a diffusion cloud chamber. The results show that the tritium concentration varies considerably: the $\mathrm{T} / \mathrm{H}$ ratios range from $10^{-15}$ to $10^{-1 \times}$ with glacial water being below detectability. The diffusion chamber technique is considerably more sensitive than counter techniques for this problem; therefore, smaller water samples and less enrichment can be used.

The tritium and neutron production in oxygen by $2.2-\mathrm{Bev}$ protons has been measured. The tritium cross section is $(27 \pm 2) \times 10^{-27} \mathrm{~cm}^{2}$, and the cross section for evaporation neutrons is $(.45 \pm .10) \times$ $10^{-24} \mathrm{~cm}^{2}$. This result indicates that a large fraction of the natural tritium is directly produced by the primary cosmic rays. The neutrons evaporated from oxygen at an angle of $90^{\circ}$ to the beam have an average energy of $6 \mathrm{Mev}$. These results are consistent with the cosmic ray evidence on the number of primaries, the amount of natural tritium and carbon-14 on the earth, and the distribution of neutrons in the atmosphere.

An attempt is being made to measure the tritium and helium- 3 in a number of recently fallen meteorites. These are being compared with Cosmotron-irradiated samples of iron and stone in order to test whether cosmic rays are constant in space and have the same intensity as those striking the earth.

\section{NEUTRON PHYSICS}

\section{The Fast Chopper}

The increased energy resolution made available by the Brookhaven fast chopper allows the measurement of the parameters of neutron resonances for many levels in a single isotope of the heavier nuclei. Below $10 \mathrm{ev}$, where the resolution is sufficiently high and the Doppler broadening is small, the parameters (width $T$, and peak cross section $\sigma_{n}$ ) for most levels can be determined from the shape of the resonance. At higher neutron energies, an area method must be used. For thin samples, the product $\sigma_{11} \mathrm{\Gamma}$ is obtained from the area above a transmission dip; for thick samples, the product $\sigma_{0} \mathrm{l}^{2}$ is obtained from such an area. The neutron width of the resonance can be computed from the measured quantity $\sigma_{11} \mathrm{~T}$. The ratio of the average of the reduced neutron width to the average level spacing $\left.\overline{\mathrm{T}^{(1)}} / \overrightarrow{\mathrm{D}}\right)$ is of considerable interest to compare with theoretical predictions. The reduced neutron width is defined as the neutron width of the resonance divided by the energy of the 
resonance. The "black nucleus" model predicts that the width-to-spacing ratio should be constant, independent of atomic weight. Recently, however, other theories (e.g., the "cloudy crystal ball" model) have been proposed which predict a variation of this ratio with atomic weight. Careful measurements have been made of the resonance parameters of nuclei above atomic weight 100 , particularly in the rare earth region, where a maximum in the ratio was predicted. Figure 7 is a plot of the experimental data for the ratio as a function of atomic weight. The theoretical curves for a 42-Mev nuclear well depth are shown for the black nucleus model and for two values of the absorption parameter $\xi$ of 0.03 and 0.05 for the cloudy crystal ball model. The experimental points exhibit a maximum, in disagreement with the black nucleus picture. However, the experimental peak near mass 160 is lower than the theoretical curve for $\xi=0.03$, the value which gives the best fit for the total neutron cross section in the Mev energy region. A larger value of the absorption parameter $\xi$ would be required to fit the low energy data.

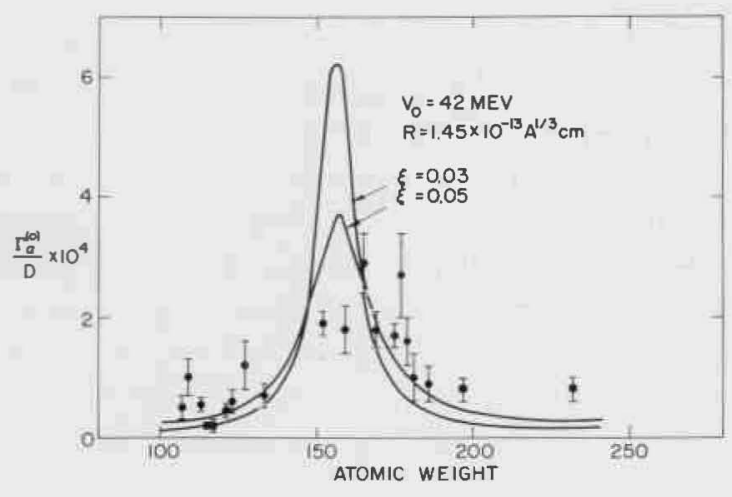

Figure 7. Comparison of the experimental values of the ratio $\Gamma_{\mathrm{n}}^{0} / \overline{\mathrm{D}}$ as a function of atomic weight with the theoretical predictions of the "black" and "cloudy crystal ball" models of nuclear scattering.

The radiation widths of many neutron resonances have been determined from transmission measurements. The radiation width $\Gamma_{\gamma}$ is obtained by subtraction of the neutron width from the total width. Parameters for several resonances in a single isotope have been measured. The individual radiation widths appear to have the same value to within about 20 percent. This consistency of the radiation width from resonance to resonance is to be contrasted with the variations of the reduced neutron widths which show fluctuations by as much as a factor of 100 . The variation of the radiation width has also been investigated as a function of atomic weight. The radiation widths decrease from $0.15 \mathrm{ev}$ at atomic weight 95 to 0.024 ev at atomic weight 238 ; with three exceptions, they fall on a smooth curve. The 6.7-ev resonance in $U^{238}$ has the smallest radiation width $(0.024 \pm 0.002 \mathrm{ev})$ yet measured. The exceptions mentioned are $\mathrm{Au}^{107}$ and $\mathrm{Hg}^{198}$ which have radiation widths about 3 times, and $\mathrm{Hg}^{199}$ which has a value about 5 times, that expected from the curve. These apparently anomalous, high values of the radiation widths for $\mathrm{Au}$ and $\mathrm{Hg}$ and the low value for $U$ can be accounted for by considerations of the neutron binding entrgy and the level density of the compound nucleus. Further measurements of radiation widths are being made in the vicinity of atomic weight 140 , i.e., near the closed shell of 82 neutrons.

Modifications in the design of the rotor of the fast chopper have been made to increase the ultimate speed of rotation. The modified rotor of Model II has spun successfully at $12,000 \mathrm{rpm}$. It has been installed at the reactor and is in routine operation at $10,000 \mathrm{rpm}$. At this speed, a neutron burst of less than 1 microsecond is obtained.

Considerable progress has been made in the development of an efficient neutron detector which has a shorter collection time than previous $\mathrm{BF}_{3}$ counters. The old counters had a collection time of about 3 $\mu \mathrm{sec}$, which is much longer than the neutron burst from the chopper. Two new detectors have been developed and have been tested with the fast chopper. Figure 8 is a photograph of a multiple proportional detector consisting of 128 counters $1 / 2$ in. in diameter housed in a single rectangular container which eliminates filling individual counters. This detector filled to $120 \mathrm{~cm}$ pressure of $\mathrm{BF}_{3}$ has a collection time of less than $0.5 \mu \mathrm{sec}$, with double the neutron efficiency of the previous detector. The flight time in the detector for neutrons with energy below $20 \mathrm{ev}$ becomes the limiting factor in determining the resolution. To overcome this difficulty, a scintillation detector has been developed which consists of an array of thin layers of an intimate mixture of fused $\mathrm{B}_{2} \mathrm{O}_{3}$ and $\mathrm{ZnS}$ mounted on type 5819 photomultipliers. The time-of-flight is negligible, and the time delays are short; the gamma sensitivity is very low, and the neutron efficiency with enriched $\mathrm{B}_{2} \mathrm{O}_{3}$ is comparable to that of the multiple proportional counter. However, for energies above $200 \mathrm{ev}$, several of the mul- 
Figure 8. Photograph of the multiple $\mathrm{BF}_{\Delta}$ proportional counter.

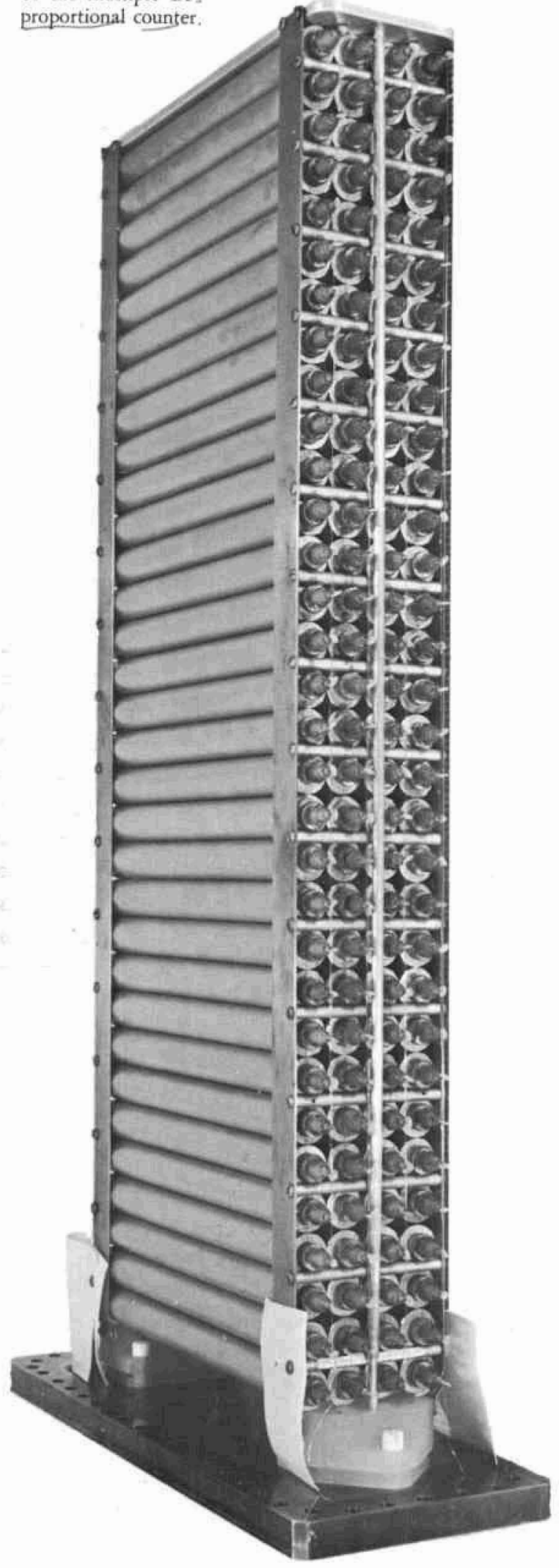

tiple proportional counters may be used to increase the efficiency without sacrificing resolution.

\section{Slow Chopper}

The results of the experiments on the magnetic scattering of slow neutrons in iron have led to the development of a theoretical description of this scattering process (by Van Hove) in terms of a correlation function in space and time. This function gives the average density distribution of the magnetic moment at a time $t^{\prime}+t$ as seen from a point where the neutron passed at time $t^{\prime}$. On the basis of this theory and the experimental measurements made at the slow chopper, a microscopic range and relaxation time have been derived for the spin system in iron below the Curie point. Measurements are now under way to see if it is feasible to determine the macrosopic relaxation time of the magnetic lattice in the vicinity of the Curie point by similar neutron measurements.

The total cross section of gaseous oxygen has been measured with the slow chopper for neutron wavelengths from 2 to $10 \mathrm{~A}$. The measurements were made in order to determine the nature of the magnetic properties of the oxygen molecule. The total cross section consists of a sum of magnetic and nuclear contributions with no interference terms since the magnetic scattering is incoherent. The nuclear contribution to the total cross section may be calculated from the free atom cross section of oxygen which is known with high accuracy. The nuclear cross section as calculated by Halpern and Appleton is subtracted from the measured total cross section to give a magnetic scattering cross section. This magnetic cross section is in very good agreement with the magnetic scattering calculated from the distribution of the magnetic electrons in oxygen.

\section{Crystal Spectrometers}

Two crystal spectrometers are being used by Brookhaven physicists for the measurement of slow neutron cross sections as a function of neutron energy. The types of cross sections which have been studied are total, scattering, and activation. The total cross sections of many elements have been studied in the energy range from 0.07 to $\sim 50$ ev. Recently, a major alteration of the collimating system of one of the spectrometers has resulted in an improvement of the resolution of this instrument by a factor of approximately six. Considerable effort has been spent on the complicated problems of analyzing the experi- 
mental data to obtain accurate Breit-Wigner parameters. Some of these methods have been programmed for the Remington-Rand 409 computer. This has permitted a more complete utilization of the data, since computations can now be easily carried out which heretofore were humanly impractical.

A study of activation cross sections has yielded isotopic assignments of several resonances in $\mathrm{Eu}$ and Ir. In addition, these measurements have yielded the curious fact that different neutron resonances populate an isomeric state in the product nucleus in varying amounts.

During the past year, the measurements of resonance scattering have been made quantitative and have yielded valuable information which supplements the results of the total cross section data. In particular, the scattering measurements yield the total angular momentum of the excited state in the compound nucleus which corresponds to the neutron resonance. Angular momentum assignments have been successfully made for resonances in $\mathrm{Te}^{123}, \mathrm{Au}^{197}$, $\mathrm{Ag}^{109}$, and $\mathrm{Ta}^{181}$.

A crystal spectrometer built for Columbia scientists has been aligned by them and is in use at the reactor with automatic operation at given energies. A study of the various factors involved in making high precision cross section measurements $(\sim 0.1$ percent) in the low energy region ( $0.1-10 \mathrm{ev})$ has been completed. These factors include stability, reproducibility, dead time corrections, and correction for higher diffracted orders. A $\mathrm{NaCl}(200)$ crystal has been shown to be superior to the LiF (111) crystal on hand on the basis of intensity and order contamination. A number of total cross sections measurements have been made. The equipment for determining the magnitude of the nonmagnetic neutron-electron interaction by measuring the variation with wavelength of the transmission of liquid bismuth is almost ready. Some scattering measurements on thin and thick gold targets are in progress. A mica crystal, with refrigerated $\mathrm{Be}$ and $\mathrm{BeO}$ filtering, has been shown to be suitable for transmission measurements to $9 \mathrm{~A}$, and will be used in studies of the effect of molecular binding on the proton cross section at long, wavelengths.

\section{0-Inch Cyclotron Experiments}

The slow neutron time-of-flight equipment of the 60-inch cyclotron has been used with the n- $\gamma$ detector technique to study the resonances in silver up to 100 ev. The results for the lowest energy resonance give a slightly higher cross section at resonance than that previously reported by others. The data for higher energy resonances are being analyzed. The study of resonances by self-absorption techniques appears to give information not directly obtainable by any other methods.

Improvements in the stability of the equipment and reliability of the monitoring have been made. The number of crystals in the $\mathrm{n}-\gamma$ detector has been increased from three to six. Changes in the ion source and pulsing equipment have resulted in a threefold increase in the counting rate without a corresponding increase in the background while the deuteron pulse width has been maintained at $0.8 \mu \mathrm{sec}$ on the external target.

\section{Counter Development}

A new large high pressure proportional counter has been constructed which has an energy resolution greatly improved over that of earlier models. Resolutions of less than 4.5 percent are now attainable with thermal neutrons on $\mathrm{He}^{3}$ and argon as the counter gas. A gold liner is being fabricated for use during high energy neutron studies.

A crystal of $\mathrm{NaI} \pm \mathrm{Tl}$ grown both by the methods of Bridgman (furnace) and Kyropoulos (pulling) was investigated under both alpha and electron bombardment as a function of temperature. It was found that, in certain cases, the pulse height per unit energy expended in the crystal was greater for the alpha particle than for the electron. This property can be important in a neutron-sensitive phosphor.

\section{SOLID STATE PHYSICS}

\section{Structure of Solids}

Structural studies on solids by means of neutron diffraction techniques have been concerned with the general fields of single crystal analysis of ferroelectric hydrogenous crystals, arrangement and dynamics of the magnetic spin system in ferromagnetics, and studies of the deviations from perfection of crystal lattices by slow neutron transmission. In addition, some development work has been done on improved diffraction techniques and facilities, particularly for single crystal work.

In a study of rochelle salt in the ferroelectric phase, experimental measurements on about 300 diffraction peaks from a deuterated crystal have been 
completed and partly analyzed. The heavy atom positions have been studied elsewhere by $x$-rays. Correlation of neutron data with such an $x$-ray study, in collaboration with Pennsylvania State University, shows also the lattice arrangement of the hydrogen atoms. From this complete structure it has been possible to determine with some accuracy the slight shifts from orthorhombic symmetry in the ferroelectric phase and thus to make deductions about the ferroelectric mechanism. The mechanism is found to concern different bonds from those which previously were postulated.

A magnetic diffraction study has been made on the changes in spin-alignment structure in $\mathrm{Fe}_{3} \mathrm{O}_{4}$ as it changes with increasing temperature from ferromagnetic to paramagnetic. Work was done partly at the Norwegian-Dutch reactor in Kjeller, Norway, in collaboration with the JENER staff and partly at Brookhaven. The magnetic diffraction peak is found to consist of two components: an elastic part which drops sharply to zero at the Curie point in accordance with the Weiss theory of intrinsic magnetization, and a diffuse peak which rises to a sharp maximum at the Curie point. The diffuse peak demonstrates that in addition to the domain structure in ferromagnetics, there exists a substructure of short range spin ordering which is a maximum at the Curie temperature but continues at higher temperatures.

In addition to work by members and associates of the Physics Department, a number of related studies, mostly on magnetic diffraction, have been carried on. These include: studies by a group from General Electric Company of the magnetic structures of $\mathrm{Bi}-\mathrm{Mn}$ and the perovskite structure $\mathrm{BaLaMnO}_{3}$, antiferromagnetic oxides $\mathrm{Cr}_{2} \mathrm{O}_{3}$ and $\mathrm{V}_{2} \mathrm{O}_{3}$ and antiferromagnetic domains in $\mathrm{NiO}$, and investigations by guests of the Laboratory of the anisotropy transition in $\alpha-\mathrm{Fe}_{2} \mathrm{O}_{3}$, other magnetic oxides, and paramagnetic scattering. Neutron diffraction research in the Chemistry Department on magnetic ferrites and ice structure is reported elsewhere.

Studies of slow neutron transmission through crystals have been continued to study deviations from perfection of the lattice. The shape of the Bragg diffraction cutoff at around $4 \mathrm{~A}$ in fine powder has been compared in detail with the shape of the Bragg peak measured at shorter wavelength with $x$-rays. Results for carbonyl iron powder, where distortion broadening predominates, and for $\mathrm{MgO}$, where particle size broadening predominates, show that in both cases there is good agreement between diffraction peak width and cutoff width. At longer wavelengths, beyond the range of Bragg diffraction, theoretical considerations have shown local lattice defects should result in an increased incoherent cross section. Measurements in this range have been applied particularly to irradiated materials, as mentioned in the following section.

A neutron spectrometer of relatively conventional design, for powder and single crystal work, was installed at the beginning of the fiscal year and is now in routine operation. A second spectrometer of similar design has been built and is being installed for use by a group from the Bell Telephone Laboratory. A considerable improvement in single crystal diffraction technique was achieved in the development of a "miniature" spectrometer of the same size as $\mathrm{x}$-ray units, which makes feasible rotation about a horizontal axis to study other layer lines. Tests of a preliminary model showed that both LiI scintillation counters and small high pressure $\mathrm{BF}_{3}$ counters were usable. A standard $\mathrm{x}$-ray goniometer has been modified for the purpose.

\section{Radiation Effects}

The work concerning the effect of irradiation on metallic materials has continued with emphasis on the effects produced in alloys. An irradiation of one week at $50^{\circ} \mathrm{C}$ in the reactor causes a drop of a few percent in the electrical resistivity of the $\alpha$-brasses. Measurements at liquid helium and liquid nitrogen temperatures and at room temperature have made possible the separation of the residual resistivity and the resistivity due to thermal scattering. Both resistivities were found to be affected by irradiation. Interpretation of these data has suggested the possibility of radiation-induced ordering. The hypothesis was tested by irradiation at liquid nitrogen temperature with a resulting increase in resistivity. This was anticipated, since the lack of atomic mobility at this temperature should suppress any tendency toward radiation-induced ordering and should increase electron scattering by the introduction of radiation defects. Experiments made to introduce order by thermal means were found to decrease the resistivity by about 1 percent. Single crystal diffraction results failed to detect any ordering. However, the amount of short range order which might have been produced and not detected by this method could account for the observed change in resistivity. An attempt has been 
made to determine the relationship between resistivity changes and variation in the integrated fast neutron flux. For example, it was found that one month's irradiation at $450^{\circ} \mathrm{C}$ produced an increase instead of a decrease in resistivity. Further work, both theoretical and experimental, directed towards a logical correlation of the various effects is in progress.

Radiation effects in insulators are being studied. $\mathrm{MgO}, \mathrm{TiO}_{2}$, and $\mathrm{MgO} \cdot 3.5 \mathrm{Al}_{2} \mathrm{O}_{3}$ show changes in optical absorption which are unstable at room temperature. Changes in the optical absorption of $\mathrm{Al}_{2} \mathrm{O}_{3}$ have been produced by both gamma and neutron irradiation. Pile irradiation produces absorption bands whose growth can be divided into a saturable part and a part linear with irradiation. Negligible recovery is produced by annealing below $200^{\circ} \mathrm{C}$ while at $600^{\circ} \mathrm{C}$ there is almost complete recovery. Saturation might be attributable to lattice recovery by virtue of radiation-induced mobility.

A study of the effects of radiation upon explosives has been initiated with Picatinny Arsenal. Work on the azides has commenced, with preliminary experiments on sodium azide showing marked color changes which differ for gamma and pile irradiation. Preliminary $x$-ray studies indicate the structure is extremely sensitive to irradiation and less to thermal and mechanical treatment.

Application of scattering theory for long wavelength neutrons by vacancies and interstitials has evolved a workable experiment for determining the number of such defects produced by pile irradiation. Direct transmission measurements extended to include wavelength dependence give the degree of aggregation of these defects. This extension has required more precise intensity measurements, which are being achieved through improved detection of long wavelength neutrons.

An x-ray facility has been set up for the use of the solid state group. Special equipment includes a low temperature cryostat which allows $\mathrm{x}$-ray data to be obtained at liquid nitrogen temperatures. This apparatus is proving particularly useful in $\mathbf{x}$-ray studies of the processes involved in radiation-induced damage, nucleation, and growth in solids when the effects are unstable at room temperature.

The liquid nitrogen irradiation facility has been operating continuously since September 1953 and irradiations have been done for the staff, for Knolls Atomic Power Laboratory, Argonne National Laboratory, and Massachusetts Institute of Technology.
Studies are continuing on radiation-induced phase transformations in tin and black phosporus. It is now possible to make irradiations and $\mathrm{x}$-ray measurements at liquid nitrogen temperatures. Measurements have been made to compare the rate of transformation of white to grey tin which was induced by irradiation and by seeding with grey tin.

Polyethylene was the principal organic material examined for the effects of ionizing radiation. Infrared measurements on the chemical bonding show the result, and a quantitative evaluation was made of changes in physical properties. Cross-linkage resulting from irradiation imparted pronounced heat stability while deleterious changes in elongation and tensile properties occurred.

Other experimental work includes the measurement of the elastic constants of $\alpha$-brass as a function of temperature in the range $80^{\circ}-293^{\circ} \mathrm{K}$.

Theoretical work on the physics of solids has continued:

1) A theory of dislocations and nuclear electrical quadrupole has been worked out to explain the reduction in intensity of the nuclear magnetic resonance signal in cold-worked copper.

2) Consideration of the effect of a variable activation energy on isothermal annealing curves has led to some general conclusions as to the form of the annealing curves and the form of the activation energy plots.

3) An investigation was made into the formation of displacements created in face-centered cubic metals by irradiation damage. Attention was centered on low energy collisions of higher symmetry. Two close competitors for the mechanism of displacement creation were found from minimum energy considerations.

4) Two chapters have been contributed by members of the group to the forthcoming book, Treatise on Rheology, Academic Press.

\section{ELECTRONICS}

The work of the Electronics and Instrumentation Division of the Instrumentation and Health Physics Department has proceeded along lines previously laid down. As in former years, activity is divided more or less evenly among four categories: research, development of new or specialized instruments, construction of more or less conventional apparatus and mainten- 
ance operations. Activities related to research and development are reported in this section, and a brief summary of construction and maintenance is included in the section on Technical Operations and Services.

Several physical research projects have been carried out in cooperation with members of other departments, chiefly on the application of coincidence and pulse height analysis techniques to studies of decay schemes. Electronics development work has been concentrated on data-handling equipment and instrumentation for magnetic measurements. Improvements have been made in a number of commonly used research instruments, and a wide variety of circuits have been designed for special applications.

The gray wedge pulse height analyzer, described in the last annual report (BNL 246 (AS-7)), has been improved. This instrument gives a direct plot on a photograph of the number of counts is pulse height. A circuit was developed to expand a section of this graph so that higher resolution might be obtained. Several gray wedge instruments were built for use throughout the Laboratory, each one incorporating different auxiliary circuits for particular types of experiments. These analyzers are used with a scintillation or proportional counter radiation detector to form a spectrometer for analysis of nuclear radiation. The cooperative research program has stimulated improvements in the instruments and development of techniques for their use, in addition to obtaining data on nine radioisotopes. The gray wedge spectrometer has also been used to study the degradation of high energy gamma rays in air (in a project partly supported by the Department of Defense) and to measure the energy distribution of the radiation inside a hollow cobalt source.

In cooperation with the Health Physics Division and with assistance from the Chemistry Department, a project was undertaken to investigate the absolute response of the ferrous sulfate dosimeter to radiation. Determinations by calorimetric and ionization chamber methods at other laboratories were widely divergent. By means of ion chambers and extrapolation techniques, it was found that the calorimeter value was correct.

As research projects and their instrumentation become more complex, data-taking becomes a serious problem. Some progress has been made in the development of automatic data-handling equipment which will save manpower and present data in a convenient form. One device, for use in a chemistry count- ing room, accumulates data from six separate experiments and prints the individual channel counts at selected intervals by means of an electric typewriter. Another system records the data from two neutron crystal spectrometers on business machine punch cards. This is an electromechanical system which accumulates counter pulses and prints the spectrometer energy setting, sample count, background count, and other essential data on the cards. The cards are then processed by the BNL computer to perform arithmetic operations and numerical integration so that all data-handling is essentially automatic up to the final answer. Other components and techniques have been investigated which may be useful in this field.

Another type of neutron spectrometer makes use of a chopper to produce bursts of neutrons. Energy is indicated by measuring the time-of-flight from the chopper to a remote detector. A 100-channel time-offlight analyzer described in the last annual report has been modified to give twice the resolution (from 1 to $0.5 \mu \mathrm{sec}$ ). A single channel time-of-flight circuit was built for use with the 18 -inch cyclotron. It makes use of a novel beam deflection tube circuit to measure times in the range of $.004 \mu \mathrm{sec}$.

Of basic importance to the new accelerator development is the focusing action of nonuniform magnetic fields. The Division has developed an instrument to measure the amount of nonuniformity of magnetic fields and to plot the field gradient as a function of position in the magnet gap. The amplifiers measure the gradient with high accuracy throughout the entire range of useful magnetic fields. Scanning of the field is automatic.

An unusually fast 10-channel analyzer was developed for use at the Cosmotron. Sorting of pulses according to height is done in a cathode-type beam deflection tube. A fast linear amplifier was developed for this application which makes use of secondary emission amplifier tubes that give about twice the speed of the best conventional radio tubes. Following the pulse sorter tube a new (British) type of sensitive trigger circuit is used in each channel, and count integrators rather than scalers store the channel counts. The analyzer will sort pulses about 10 times faster than previous pulse analyzers $(0.1 \mu \mathrm{sec}$ compared with $1.0 \mu \mathrm{sec}$ resolution).

Ionization chambers with sensitive electrometer circuits are used to measure the intensity of radiation fields. The small chambers for measuring high fluxes of radiation have been redesigned and standardized 
for use in calibrating the kilocurie sources produced in the Brookhaven pile. A pair of balanced ionization chambers and an electrometer circuit was built for accurate comparison of an unknown to a reference source. Precise measurements may be made with this apparatus which enable determination of the rate of decay of relatively long-lived radioisotopes. An ionization chamber and logarithmic electrometer circuit were constructed for measuring the activity and the rate of decay of short-lived isotopes produced in the pile. An ionization chamber type of radiation survey instrument has been redesigned. It has a wide range of scales, high stability and linearity, yet it uses only two battery types and has long battery life. The cheap plastic chamber may be discarded if it should become contaminated. Reliable ionization chamber monitor and alarm units were installed at the Hot Laboratory.

The following are the more interesting of the many projects or instruments provided to various scientists throughout the Laboratory: an oven for applying a thermal transient to a sample, and a sonar type of circuit for measuring velocity of sound in the sample were built for studies in solid state physics; a fine focus, high velocity electron gun which can be precisely aligned was constructed for studies of a strong focusing accelerator tube; a high voltage supply was designed for operating several high gain photomultiplier tubes used in ultra fast coincidence work at the Cosmotron; a circuit used for studying the density of biological slides through a microscope was rebuilt to provide a comparison of the densities at different locations on the slide; a more sensitive detector was provided for a densitometer in the Chemistry Department; a servo mechanism and timing circuits were designed for use with a sensitive pressure meter enabling the chemists to develop a new method of analysis of the composition of lighter gases; an oscillographic device was assembled to check the operation of camera shutters for the photographic group; circuits were developed for monitoring the beam of the Cosmotron and for calibrating the monitoring system.

\section{ACCELERATORS}

The most outstanding event of the past year at Brookhaven was the approval by the Atomic Energy Commission of a proposal for the construction of a
25-Bev strong-focusing proton synchroton. Very preliminary design studies of possible strong-focusing accelerators were undertaken after the discovery of the strong-focusing principle in 1952. Early in the past fiscal year the general features of such a machine were described and the Atomic Energy Commission granted its approval in January 1954 for Brookhaven to proceed with the development, design, and ultimate construction of a 25-Bev proton synchrotron at a total cost of $\$ 20,000,000$. To pursue this important development and construction program, an Accelerator Development Division was formed. Its rapid growth during the past fiscal year and its increased duties and responsibilities have caused it to be made into a full-fledged department as of July 1954 with Leland J. Haworth as Chairman, G. K. Green as Deputy Chairman, M. M. Small as Construction Manager, and C. E. Falk as Administrative Scientist.

The other Brookhaven accelerators, namely the Cosmotron, 60-inch cyclotron, 18-inch cyclotron, and three electrostatic accelerators, are now operated within the departments using them. The Cosmotron Department is not only responsible for maintenance and operation of the Cosmotron but also participates in the high energy research program along with other departments. The two cyclotrons and the electrostatic accelerator operated and maintained by the Physics Department are primarily for the use of the physicists but also for research in biology, medicine and chemistry. Although the cyclotrons have undergone periods of major repair and revision, they are now performing in a satisfactory manner and are contributing to the research programs of the Laboratory. The long-delayed modification program for the electrostatic accelerator was initiated during the year and highly successful operation has been achieved at even higher energies than sought for through the modification. Considerable time was spent in the study of electrostatic accelerators as such during this period rather than directing its use immediately toward experimental research.

\section{COSMOTRON}

The Cosmotron Department has in its first full year of operation been able to concentrate its greatest effort on research activities because sustained efficient operation of the machine demanded comparatively little manpower. Since experimenters have always requested more research time at the Cosmotron than 
was available, a great effort was made to arrange matters so that maximum use was made of the available time. Usually two or more experiments are run at the same time - and occasionally as many as five run concurrently. Four universities are operating their own cloud chambers at various positions around the Cosmotron. In addition, outside physicists and their graduate students have been using the Laboratory cloud chambers to obtain photographs of high energy events which are then studied at their home institutions. The photographic emulsion program operates in much the same way, with members of the Laboratory staff assisting visiting physicists in exposing to high energy beams emulsions which are then examined in the visitors' own laboratories.

\section{Operation}

Since January, proton energies up to the design value of 3 Bev have been available on a routine basis and the maximum number of protons per pulse is now about five times the number of a year ago. Operation continues on a five-day week, 8:30 a.m. to midnight, with operation on Saturdays scheduled whenever this day is not needed for maintenance or rearrangement of experimental apparatus and shielding. In addition, a one-week shutdown is scheduled about every seventh week for modifications and maintenance too extensive to be performed over a weekend. The operational efficiency of the Cosmotron can be judged from the fact that once scheduled, an experimental run has about a 75 percent chance of taking place. The 25 percent lost time is due to failures of various kinds among all the components. One exception to this general pattern of operation was caused by a leak in one of the rubber blankets which cover the upper and lower surfaces of each of the four vacuum chambers. This leak could not be repaired in place and the quadrant was removed to permit a new upper and lower rubber blanket to be installed. This repair took one month.

To make full use of the research possibilities of the Cosmotron a great deal of auxiliary equipment is necessary, and for the past year a major part of the time of the engineers assigned to this Department was spent in designing and procuring this type of gear. The following are major additions in this category. A third 14-ton electromagnet similar to the two already in use was constructed; these magnets have proved to be extremely useful in analyzing the various high energy beams which emerge from the shielding. The technique of constructing both metal and styrofoam liquid hydrogen targets has been pursued and several styrofoam targets have been constructed and used. The building to house the dc generators needed to produce magnetic fields for cloud chambers and analyzing magnets is now complete and four $\mathrm{dc}$ generators ranging from $12 \mathrm{kw}$ to $100 \mathrm{kw}$ output have been installed together with their power distribution systems. Each of these generators is controlled and regulated from a remote and portable console so that experimenters can control their own magnetic fields from a point near their experimental area.

It is very important but at the same time quite difficult to measure quantitatively the number of protons contained in each pulse. Now after a year of development, a circulating beam monitor is available which has an absolute accuracy of about \pm 20 percent and a relative accuracy of \pm 2 percent. This device is used regularly by experimenters and operators alike. The monitor has confirmed the earlier evidence that only about 20 percent of the protons captured initially is present at the end of the acceleration cycle. It is believed the remaining 80 percent is lost by inadequacies in the accelerating of system and consequently an improved system is being designed and constructed which it appears should reduce this loss considerably. Any further improvements in the proton beam will however make additional shielding necessary as the present radiation levels in several critical regions are close to the maximum that can be tolerated. More shielding in the form of additional concrete blocks to be placed at strategic points is planned.

A system of relays has been devised to increase the flexibility of the Cosmotron so that experiments requiring different operating conditions can run concurrently. With this system it is possible to establish two sets of operating conditions, each set having its proton energy, its own proton intensity and, by means of a pneumatically actuated ram, its own target. Either of these two sets of conditions can be imposed on any pulse by means of an electrical signal.

The system is particularly effective in connection with the large expansion cloud chambers; these cloud chambers cannot use pulses more than once every 1 to 5 minutes and would thus be very wasteful of Cosmotron time if they had to operate solo. With the relay system experiments in need of the maximum pulsing rate proceed with proper conditions 
(as regards energy, intensity and target) and once every 2 minutes or so they are deprived of one pulse of protons which is then adjusted to the new set of conditions demanded by the cloud chamber. The irradiated chamber is expanded, the resulting events photographed and the cycle of events repeated. It is, of course, possible to have more than two sets of operating conditions controlled by the relay system but arrangements of this complexity have not been tried.

For many experiments an intense, full energy external proton beam is a necessity and a method to achieve this has been under development for a year. An electromagnet (gap 1 in. high by 3 in. deep by 30 in. long) is located in one of the four straight sections. The middle of the gap will be some 4 or 5 inches inside the normal trajectory of the circulating proton beam. At the end of the accelerating cycle the beam is made to pass through a target of such thickness that the energy lost by the protons displaces their orbit inward 4 or 5 inches in a single turn, and causes thus them to enter the gap of the electromagnet. There the protons suffer a deflection of about $3^{\circ}$ outward and, because of this deflection, the protons will emerge outside of the next straight section where a second electromagnet will be located to deflect the protons an additional $10^{\circ}$, thus to produce a convenient external proton beam. The effectiveness of this scheme has been tested in the Cosmotron by use of apertures which correspond to the proposed electromagnet. Experiments show a recovery of about 10 percent of the circulating beam is to be expected. The first electromagnet is complete and will be tested soon; the second electromagnet is in the design stage.

While work continues on achieving an intense external proton beam, use is being made of a weaker external beam obtained by a simple method. It has been found that the frequency control of the accelerating if is capable of retaining the circulating proton beam while the Cosmotron's magnetic field reaches a maximum and begins to diminish. At this stage the accelerating of is turned off and the diminishing magnetic field of the Cosmotron causes the protons to spiral out. Some of the protons then pass the critical point where blowup might be expected and are thrown out of the gap like mud off a wheel. Proton intensities outside the concrete shield amount to several hundred per $\mathrm{cm}^{2}$ per pulse and this is sufficient for cloud chamber work and total absorption experiments.

\section{ALTERNATING GRADIENT SYNCHROTRON}

The Accelerator Development Department (ADD) carries out the design and development of improvements in the accelerator field. The major effort of the Department is now concentrated on the AGS project, i.e., the construction of a 25-Bev strongfocusing proton synchrotron. This project was approved by the Atomic Energy Commission at the turn of the calendar year after a detailed design study of strong-focusing synchrotrons had been submitted. An artist's sketch of the proposed machine is shown as the frontispiece of this report, and a preliminary table of proposed design parameters for the machine is given in Table 1.

Table 1

Preliminary design parameters for the 25 - to $35-\mathrm{Bev}$ alternating gradient synchrotron

\begin{tabular}{ll} 
Energy at 10,000 gauss & $24.7 \mathrm{Bev}$ \\
Energy at 12,000 gauss & $29.8 \mathrm{Bev}$ \\
Energy at 14,000 gauss & $34.9 \mathrm{Bev}$ \\
Injection accelerator & $\mathrm{Linac}$ \\
Injection energy & $50 \mathrm{Mev}$ \\
Transition energy & $7.5 \mathrm{Bev}$ \\
Radius of curvature & $280 \mathrm{ft}$ \\
Field gradient index, n & 348 \\
Betatron oscillation frequency, v & 8.75 \\
Rise time & $1 \mathrm{sec}$ \\
Repetition rate & $20 / \mathrm{min}$ \\
Circumference & $2647 \mathrm{ft}$ \\
Over-all diameter & $844 \mathrm{ft}$ \\
Weight of steel & $3000 \mathrm{tons}$ \\
Weight of copper & $-350 \mathrm{tons}$ \\
Harmonic order & 12 \\
Frequency range & $1.4-4.5 \mathrm{Mc}$ \\
No. of ferrite cavities & 24 \\
No. of power amplifiers & 12 \\
Energy gain per turn at 14,000 gauss & $96.4 \mathrm{kv}$ \\
Peak rf per cavity at 10,000 gauss & $5750 \mathrm{v}$ \\
Peak rf per cavity at 14,000 gauss & $8000 \mathrm{v}$ \\
\hline
\end{tabular}

The program of the AGS project calls for an inital 1-2 year period of component development which can be broken up roughly into three major activities: development of the strong-focusing magnet which will guide the protons around the synchrotron, development of the radiofrequency system which will provide acceleration for the particles, and development of a 50-Mev linear accelerator which is to inject the protons into the AGS. Coupled with these efforts are an intensive theoretical investigation of proton orbit dynamics and the construction of a 
working synchrotron analogue which will accelerate electrons. This electron analogue, the construction of which is well along, is being built to study problems associated with phase transition and resonances. It should be emphasized that this machine is not a model of the 25-Bev synchrotron but a device designed along the simplest engineering lines which will make possible the study of the above mentioned phenomena.

With the approval of the AGS project, the Accelerator Development Department underwent a considerable expansion in personnel as well as in housing facilities. Thus, the number of ADD personnel increased to 63 , of which 26 are on the scientific and engineering staff. Most of the development work is now being carried on in six barracks buildings which house offices, drafting rooms, laboratories, a machine and electronics shop, and an administrative section which handles purchasing, expediting, contractual and fiscal affairs. The electron analogue is being constructed in the Cosmotron test building since this was the only available space of sufficient area which would house this machine. All these housing facilities are temporary and will be replaced by permanent buildings which will be part of the ADD building complex that will also house the strong-focusing synchrotron. A search for an architect-engineering firm has been made in order to get the building design completed as quickly as possible, and a contract with Stone \& Webster Engineering Corporation is being negotiated.

As in the previous year, the Brookhaven group worked in close cooperation with personnel of other multibillion-volt accelerator projects. Thus, members of the staff spent some of their time at the Radiation
Laboratory of the University of California, the CERN laboratories of the European Research Council, and with the Midwestern Accelerator Group. Furthermore, subcontracts for accelerator development to the Massachusetts Institute of Technology and Princeton University were administered.

\section{Electron Analogue}

As mentioned before, the construction of the analogue was undertaken to provide information on phase transition and resonance problems. It is expected that the knowledge gained from the analogue will make the design of the large proton synchrotron more efficient and economical. This machine uses a 2-Mev electron Van de Graaff as an injector and 80 electrostatic electrodes as guide and focusing lenses. A large number of quadrupole, sextupole, and octuple lenses are interjected along the electron path to make changes in the guide field possible. The lenses themselves are housed in 16 vacuum chambers which are connected by 7 -in. flexible joints. Sixteen 10-in. mercury diffusion pumps will be used to maintain the required pressure of about $5 \times 10^{-7} \mathrm{~mm}$ of mercury. The electrons are accelerated from an initial energy of $1.5 \mathrm{Mev}$ to a final energy of about $10 \mathrm{Mev}$ by means of a single ferrite cavity. Phase transition occurs at 2.5 Mev. The diameter of the analogue is $45 \mathrm{ft}$, and its construction was undertaken in the Cosmotron test building. Figure 1 shows the foundation ring for the analogue with the Van de Graaff in the background. Table 2 outlines the major design parameters of the machine.

At this time most components of the analogue are in the construction and fabrication stage. It is expected that assembly will be completed by early

Figure 1. Foundation of the electron analogue in the Cosmotron test build ing. The injector electron Van de Graaff can be seen in the background.

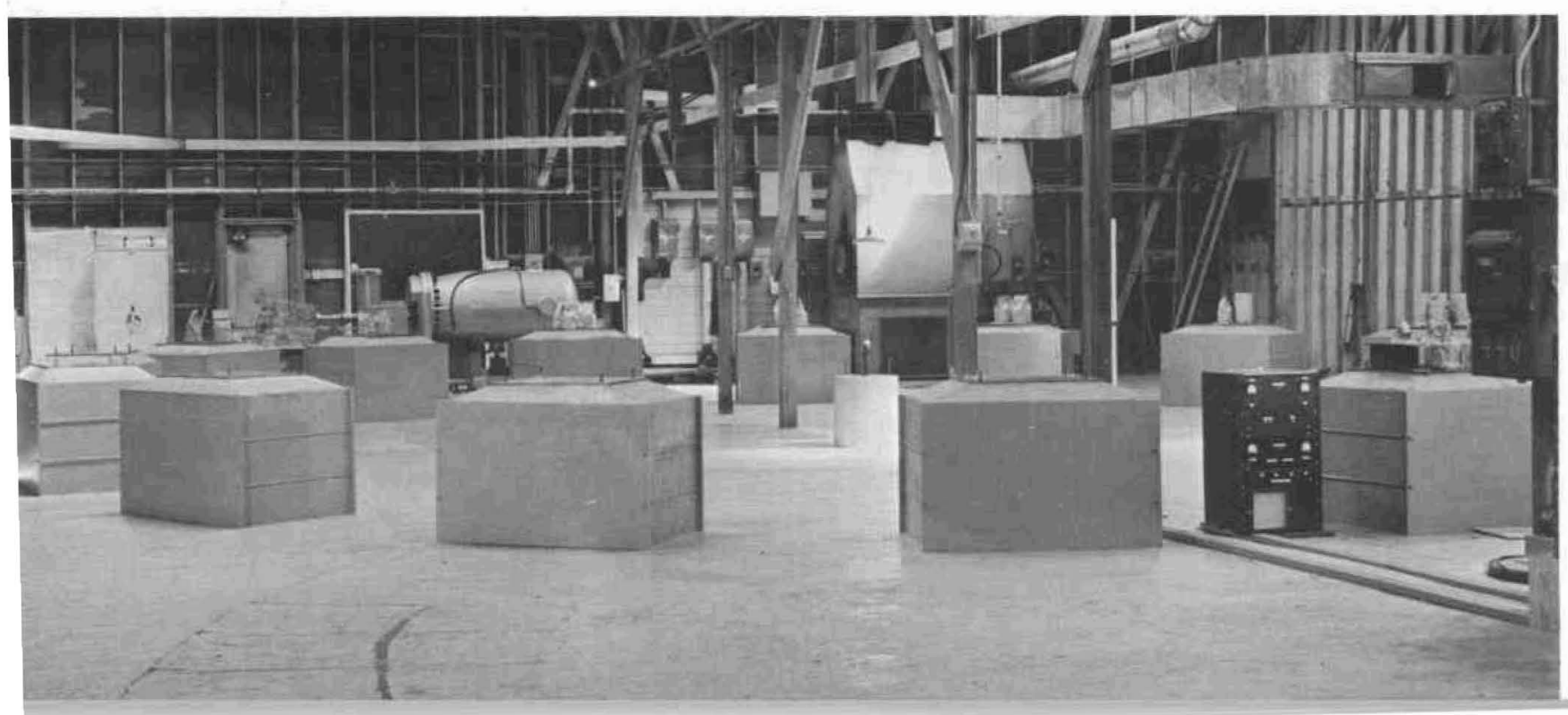


Table 2

Design parameters for the electron analogue

Radius of curvature

Diameter

$15 \mathrm{ft}$

Guide electrode aperture

$45 \mathrm{ft}$

Transition energy

Injection energy

Maximum energy

Field gradient index, $\mathrm{n}$

Number of double unit cells

Rise time

Rf frequency

Energy gain per turn

Repetition rate

Pressure

Betatron oscillation frequency, $v$

Maximum possible change in

betatron frequency, $\Delta v$

Quadrupole lens aperture

Sextupole lens aperture

$(0.8 \text { in. })^{2}$

$2.5 \mathrm{Mev}$

$1.0 \mathrm{Mev}$

$10.0 \mathrm{Mev}$

225

40

$5.10 \times 10^{-3} \mathrm{sec}$

$7 \mathrm{mc}$

$\sin \phi_{0} \mathrm{ev}=150$ or $300 \mathrm{ev}$ $6 / \mathrm{sec}$

5 or $10 \times 10^{-7} \mathrm{~mm}$ of $\mathrm{Hg}$

$63 / 4$

$1 \frac{1}{2}$

$(1.2 \text { in. })^{2}$

$(1.2 \text { in. })^{2}$

fall and that the machine will be in operation early in 1955 .

\section{The AGS Project}

The study of strong-focusing synchrotrons, which had been started in the previous year, resulted in the determination of a set of preliminary design param eters for AGS machines in the 15 - to $100-\mathrm{Bev}$ range. Close examination of these parameters revealed that a 25 - to 35-Bev alternating gradient synchrotron would be most appropriate for Brookhaven. Consequently, a proposal for the construction of such a

machine was made to the Atomic Energy Commission. Early in January this proposal was approved.

The group then began the initial phase of the construction program that is estimated to take about 6 years. At the same time a gradual expansion of personnel was initiated to bring the number of people necessary for machine construction to an eventual peak of 125 . This increase in personnel included the acquisition of the project's buyer, fiscal agent, contractual officer, and other administrative personnel.

It is estimated that the first year and a half of effort will go into the development of final machine design parameters. Consequently, the initial period was used in the development of the magnet, the radiofrequency system, and the injection linear accelerator. These studies were guided by an intense theoretical investigation of the orbit dynamics of protons in strong-focusing synchrotrons.

\section{The Magnet}

The magnet group spent a considerable amount of their time on the development of measuring and calibration equipment. This equipment has now been sufficiently developed to make possible dc magnetic measurements, to accuracies of at least 0.3 percent. The equipment developed and built includes a constant gradient coil, called the Gradhelm, which is being used as a gradient standard (see Figure 2). It has been shown that the gradient in the center area $\left(\sim 7\right.$ in. $\left.^{2}\right)$ of this coil is constant to better than 0.1 percent.

Figure 2. Constant gradient Gradhelm coil with gradmeter in position for calibration.

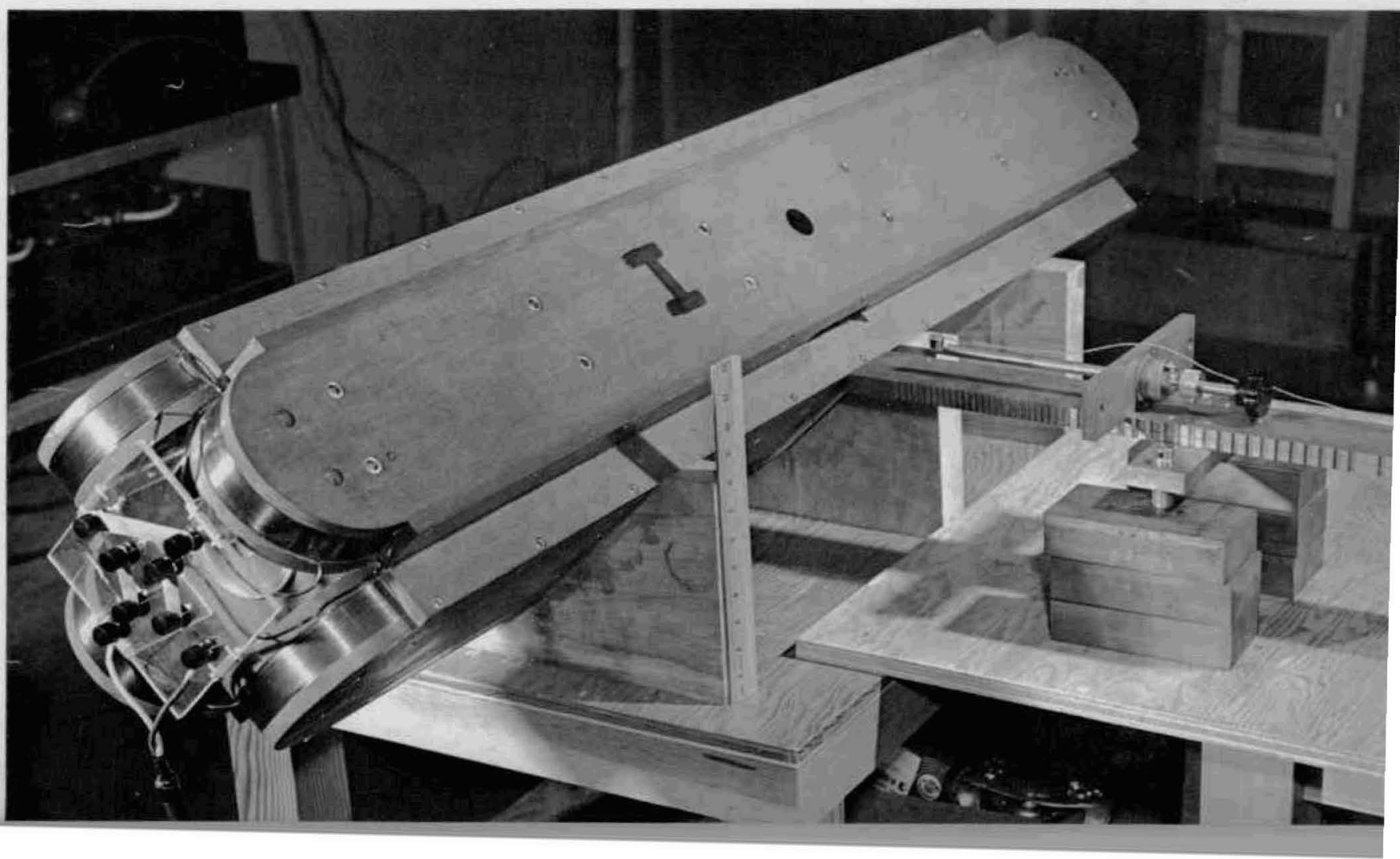


The measuring equipment has been tailored to particular requirements. In general it was desirable to determine the following parameters of model dc magnets: magnetic field as a function of coil current, the gradient across the magnet gap in the median plane at various coil currents, the gradient at points off the median plane, the magnet end effects on gradients and flux in the magnet iron.

As can be seen, it is essentially necessary to be able to measure the field and its first derivative. Fields are generally measured by using accurately wound flip coils and observing induced voltages on fluxmeters. This is a standard method which has been used for a long time. However, the measurement of strong magnetic field gradients presented problems which had not been encountered before. Two different devices were developed to measure this all-important magnetic parameter. One of these is the Gradmeter (see Figure 2), which consists of a coil mounted on a 3 -ft long glass rod which oscillates in the unknown field. By means of a self-induced reference field as well as servo and manually controlled attenuators, it is possible to obtain a frequency and amplitude independent signal from the oscillating coil and compare it to the signal from a coil in the reference field. The final comparison signal is directly proportional to the gradient. The vibrating coil can be drawn smoothly across the magnetic field under study and the gradient signal automatically recorded as a function of coil position on an $x-y$ recorder. This device greatly reduces the time necessary for the gradient versus $\mathbf{R}$ measurements; one of these runs can be completed in about 4 minutes. Another device called the $\mathrm{n}$-meter directly measures the field gradient index $\mathrm{n}$ which is defined as $\mathrm{n}=$ $(R / B)(d B / d R)$, where $R=$ radius of orbit and $B=$ magnetic field. This involves the measurement of the ratio $d B / d R$ and $B$. The $n$-meter consists of three coils which are located on a rigid mounting. Two of the coils are matched and connected in such a way as to have induced voltages in opposition. When the mount is rotated $180^{\circ}$ in the unknown field, the difference signal from these two coils is proportional to the field gradient. The signal from the third single coil is proportional to the field. By balancing the two signals on a potential divider, it is possible to measure directly a ratio of $\mathrm{dB} / \mathrm{dR}$ to $\mathrm{B}$. It is of interest that gradient measurements made with these two devices, which are calibrated by independent methods, have shown good agreement (see Figure 3 ).

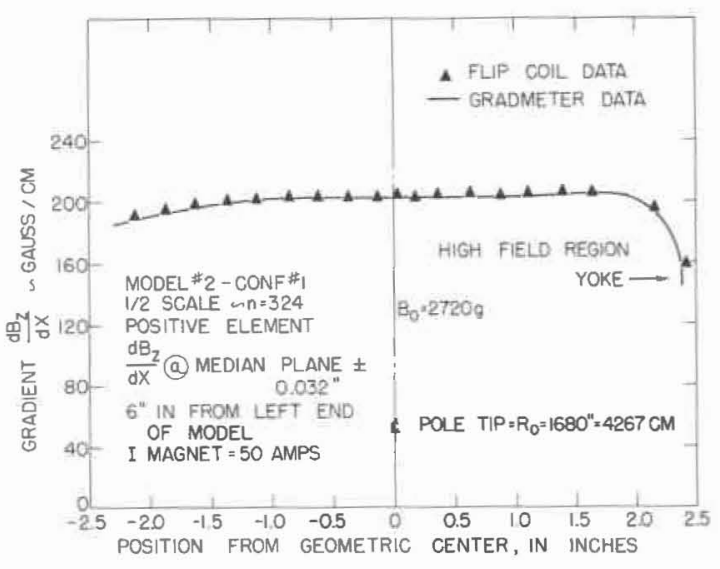

Figure 3. Gradient as a function of position in dc magnet model no. 2, configuration no. 1.

The above equipment has been used to obtain information from two dc model magnets (see Figures 3 and 4). This information has been analyzed and is now being used in the design of a dynamic model magnet. It is hoped that the results from measurements on the dynamic model will lead to the design and construction of a prototype sector of the strong-focusing magnet.

\section{The Radiofrequency System}

Development work for the radiofrequency system concentrated on the accelerating cavity and the power amplifier that will drive it. It was decided to use ferrite accelerating cavities and an extensive experimental study of various ferrites was undertaken. One ferrite was found which met all design requirements. A model cavity was then designed and the 500 pounds of ferrite that are necessary for this model were ordered.

A study of power tubes and amplifier circuitry showed that it would be possible to have fewer accelerating stations than had been anticipated earlier and yet use only air-cooled tubes. With the introduction of some $10-\mathrm{ft}$ long straight sections in the AGS it was decided to have ferrite cavities, connected in parallel, in one straight section and have them driven by one power amplifier. The latest design favors 24 accelerating cavities, each at 8000 volts, located in 12 positions around the magnet ring. Two cavities per straight section were chosen so that the shields of the cavities could be used as dc biasing conductors in a figure-eight configuration. This biasing method achieves almost perfect decoupling of the $\mathrm{dc}$ and $\mathrm{rf}$ windings. 


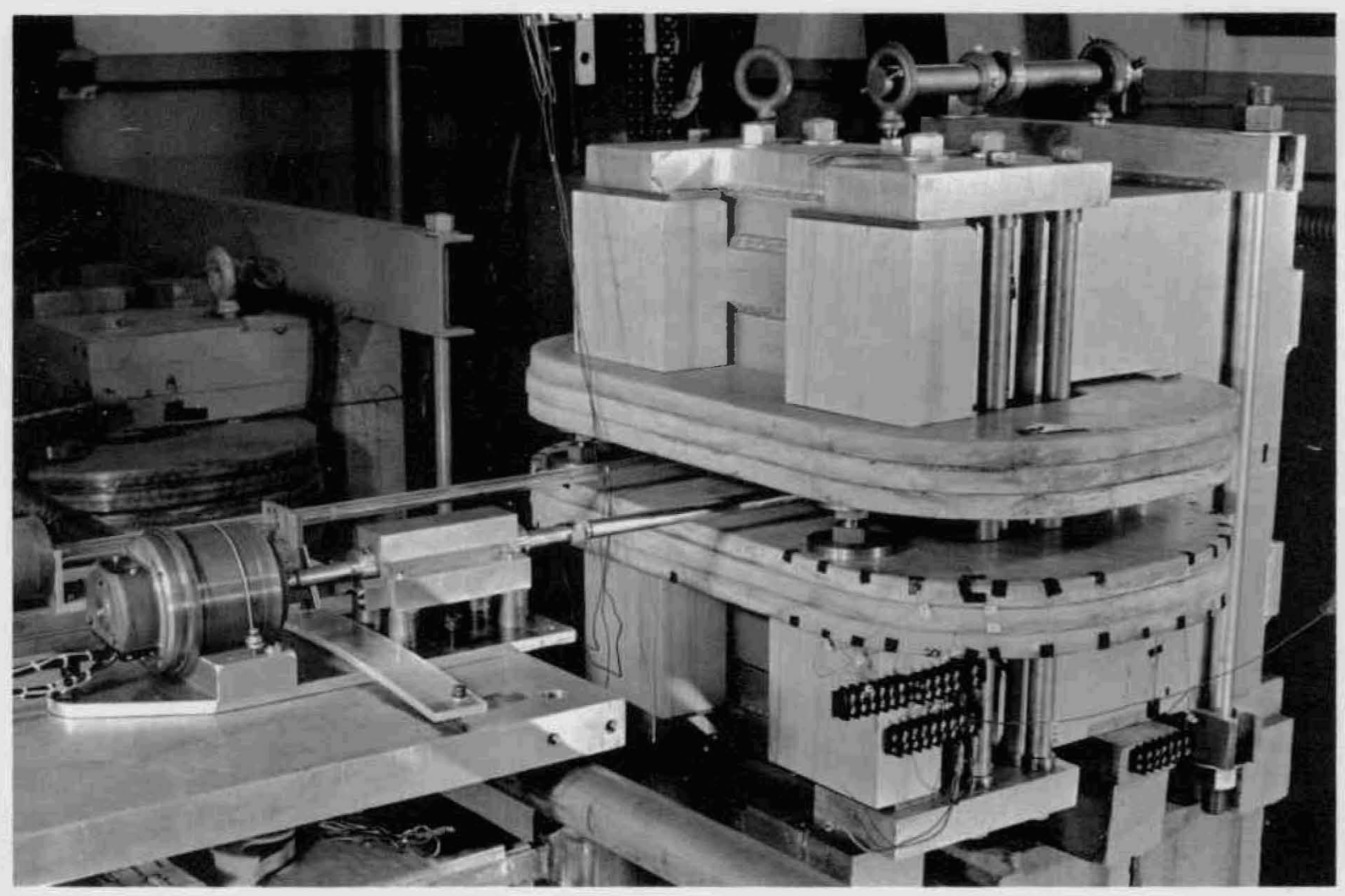

Figure 4. Dc model magnet no. 2, configuration no. $1, \mathrm{n}=324$, with gradmeter and $\mathrm{n}$-meter in measuring position.

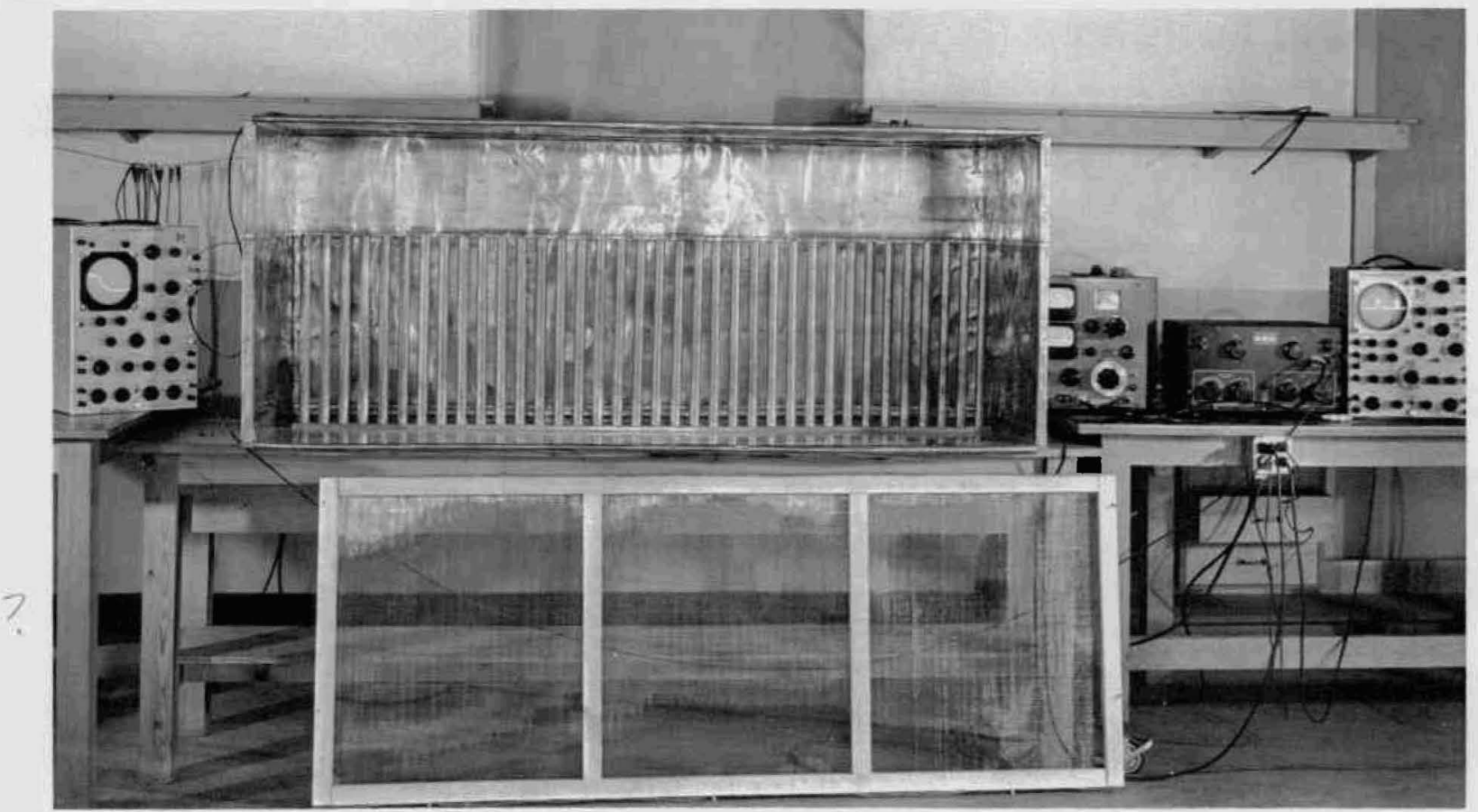

Figure 5. Thirty-six section mockup of "organ-pipe" scheme of if power application to linac drift tubes. 


\section{Linear Accelerator}

The linear accelerator to be used as an injector for the synchrotron will have to produce protons of $50 \mathrm{Mev}$. It will have to provide better energy control and less angular beam divergence than most present experimental linacs. Consequently, a considerable development of linac technique is necessary to meet these qualifications. Up to now, the linear accelerator group has investigated mostly the problem of applying rf voltage to drift tubes. A number of possible schemes were considered and many of them studied by testing experimental mockups (Figure 5). At the same time a PIG-type ion source and a 100-kev preaccelerator were designed and constructed. The beam coming from this source is intended to go through about $30 \mathrm{ft}$ of a model linear accelerator. Focusing is one of the main problems to be solved. It is hoped that it can be accomplished by means of permanently magnetized strong-focusing pipes. Several magnetic materials are being tested for their applicability to this problem. A 50-kev electron gun setup has been constructed and the focusing abilities of these magnetic pipes are being tested by having electron beams pass through them.

\section{Theoretical}

The theoretical investigations of proton orbits in alternating gradient machines continued. Most of the mathematical problems were computed at the AEC Univac installation at New York University and produced the following results.

Studies of the effects of nonlinear terms in the magnetic field showed that up to 10 percent of cubical terms could be tolerated; these terms might even increase the stability of the machine because of their tendency to broaden and weaken resonances.

It was shown that the order of magnitude of coupling between vertical and horizontal betatron oscillations due to field inhomogeneities is small enough not to affect the stability of proton orbits.

Investigations of the change of behavior of equilibrium orbits which result from inhomogeneities in the field revealed that these field irregularities not only would produce physical displacements of the equilibrium orbits but could actually produce orbit multiplicities.

A detailed study of the effects of magnet misalignments was made. Errors in radial alignment are about ten times as harmful as errors in magnet length or in rotational and azimuthal alignment.
Calculations on aperture requirements resulted in an analysis of factors such as betatron oscillations at injection, linac emittance, gas scattering, and magnet misalignment which tend to limit the aperture.

Over the year 360 hours of Univac computer time were used, most of which were concentrated into the last few months. The computing program is continuing, and it is expected that about 800 hours of Univac time will be used during the next year.

\section{0-INCH CYCLOTRON}

During the past year, the cyclotron has been improved in performance and particularly in reliability. A wide variety of targets have been irradiated and several types of experiments have been done with the external beam. During the last quarter of 1953 , schedules of operation were met with better than 90 percent reliability. During the first quarter of 1954 , a new set of dees was installed. These new dees largely eliminated the many troubles caused by inadequate cooling of the old dees, including a long warm-up time, serious drifts in operating conditions and in beam energy. Since the change of dees, the cyclotron has operated at $50 \mu \mathrm{a}$ of $20 \mathrm{Mev}$ deuterons, $30 \mu \mathrm{a}$ of $40 \mathrm{Mev} \alpha$ particles, and $80 \mu \mathrm{a}$ of protons (accelerated and measured as hydrogen molecular ions). All these currents are for a deflected beam on a useful target area. So far, no attempt has been made to increase the current beyond these levels, which are adequate for present requirements. A new deflector has been installed which should be an improvement but its limitations have not yet been determined. Changes in the ion source have increased the pulsed beam by a factor of 3 .

A program for bringing the external beam to a focus at a considerable distance from the cyclotron is being pursued. Preliminary measurements indicate that with focusing magnets now under construction a satisfactory beam will be obtained.

\section{8-INCH CYCLOTRON}

A deflected beam of $1 \mathrm{ma}$ of $2.8 \mathrm{Mev}$ protons can now be consistently obtained in the rarget box of the 18-inch cyclotron. The larger beam current is the result of improvements in the efficiency and stability of the oscillator and improvements in the ion source and deflection system.

Preliminary experiments have been carried out on 
the use of zirconium-tritium targets for the production of neutrons. These experiments have indicated the need for improved cooling of the targets to withstand the available beam current and the possibility that some material other than tungsten should be used as the base for the zirconium film.

Extensive experiments have been carried out on the "phase-bunching" of the proton beam in the cyclotron. The data show the "bunch" is between 1 and 3 millimicroseconds long. This time was determined by observation of the gamma rays produced by the protons on a target of aluminum or copper. The extremely short duration of the beam-bunch will make it possible to measure neutron energies by time-of-flight methods to a precision of about 1 percent. Work is being continued to study the effect of various machine parameters on this phenomena. The instrumentation developed in this work will also be useful for the study of isomeric states having decay times of the order of 1 millimicrosecond.

\section{RESEARCH ELECTROSTATIC GENERATOR}

During the past 18 months, the research electrostatic generator, purchased from the General Electric Company, has been down for a complete overhaul which is now approaching completion. The purpose of this overhaul, as reported previously, was (1) to increase the upper voltage limit and ion beam output, (2) to improve the general operational reliability, (3) to increase the general experimental usefulness by minimizing background radiations, by making helium ions available in addition to protons and deuterons, and by providing multiple beam outlet pipes to permit easy switching from one experimental apparatus to another.

A very stable belt charging system has been achieved by use of individual stabilizing resistors on all spray points, inside spray, and a split inductor system.

Observations of the spontaneous loading which can appear in an acceleration tube under suitable conditions of residual pressure and gradient led to the discovery of the important role that can be played by ionization currents generated in the high pressure insulating gas by $\mathrm{x}$-rays arising from back accelerated electrons in the acceleration tube. Such currents impose an extraneous load on the machine and can distort the desired uniform gradient along the column and thereby promote sparking. After this dis- covery, a beryllium-lead geometry was incorporated at the anode end of the tube to minimize the production of $\mathbf{x}$-rays by back-streaming electrons and to maximize the absorption of those $\mathrm{x}$-rays which are produced.

Initial tests have been conducted recently with an ion beam. A total ion beam of 20-30 $\mu$ a has been accelerated to an energy of $3.5 \mathrm{Mev}$ with a free-running voltage stability of about 0.3 percent. The $x$-ray level beside the tank was about $25 \mathrm{mr} / \mathrm{hr}$ which is about one hundred times smaller than that previously encountered with a lower voltage and beam current. It is expected that within about 2 months the machine will again be available for research.

\section{CHEMISTRY}

The research program of the Chemistry Department has continued along lines previously established. It is discussed below under the headings: Nuclear Chemistry; Chemistry and Applications of Isotopes; Chemical Effects of Radiation; Chemical Effects of Nuclear Transformations; Molecular Structure and Solid State.

\section{NUCLEAR CHEMISTRY}

A large part of the Department's efforts in the field of nuclear chemistry was devoted to studies of proton-induced nuclear reactions with the Cosmotron. In order to express the yields of such reactions in terms of absolute cross sections it was necessary to devise a method for the determination of the intensity of the proton beam striking a target. Since no external proton beam of appreciable intensity is available yet, all irradiations had to be performed in the circulating beam, and accurate intensity measurements are difficult under these conditions. The method chosen is based on the observation that decay curves of copper foils activated by short proton bombardments have almost identical shapes for all bombardment energies from 0.4 to $3 \mathrm{Bev}$. In the time

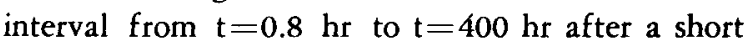
( $<5$-min) irradiation, the gross activity $A_{t}$ may be represented approximately by $A_{t}=k^{-1.2}$, where $k$ is a constant. This indicates that an essentially statistical assembly of half-lives is formed and, although the yields of individual radioactive products may be energy-dependent, it seems plausible that the cross sec- 
tion $\sigma^{*}$ for the production of atoms decaying between $0.8 \mathrm{hr}$ and $400 \mathrm{hr}$ may represent a constant fraction of the total inelastic cross section of copper. With this assumption and a measurement of $\sigma^{*}$ at $450 \mathrm{Mev}$ relative to the cross section of the reaction $\mathrm{Al}^{27}(\mathrm{p}, 3 \mathrm{pn}) \mathrm{Na}^{24}$ which is known to be $10.8 \mathrm{mb}$ at $450 \mathrm{Mev}$, it was possible to measure proton beam intensities at the higher energies. The results are believed to be accurate to \pm 30 percent, and check within less than 20 percent with the electrical measurements of the circulating proton beam.

The excitation function of the $\mathrm{Al}^{27}(\mathrm{p}, 3 \mathrm{pn}) \mathrm{Na}^{24}$ reaction has been determined in terms of the grosscopper-activity method described. The cross section of this reaction decreases only very slowly and essentially linearly with increasing energy, from $10.8 \mathrm{mb}$ at $450 \mathrm{Mev}$ to $8.2 \mathrm{mb}$ at $3.0 \mathrm{Bev}$. Because the $15 \mathrm{hr}$ $\mathrm{Na}^{24}$ activity is readily measured in proton-bombarded aluminum without chemical separation, aluminum foil is routinely bombarded along with any target, and the $\mathrm{Na}^{24}$ activity serves to monitor the beam intensity.

The slow decrease in cross section with increasing energy found in the case of the $\mathrm{Al}(\mathrm{p}, 3 \mathrm{pn})$ reaction appears to be rather characteristic of relatively simple reactions in light nuclei. Figure 1 shows the excitation function of the reaction $\mathrm{C}^{12}(\mathrm{p}, \mathrm{pn}) \mathrm{C}^{11}$ obtained from the ratio of $\mathrm{C}^{11}$ activity in polyethylene foils to $\mathrm{Na}^{24}$ activity in aluminum foils. The production of $\mathrm{Na}^{22}$ and $\mathrm{F}^{18}$ from aluminum and that of $\mathrm{Be}^{7}$ from carbon have similarly flat excitation functions. On the other hand, reactions requiring greater excitation energy, such as the formation of $\mathrm{Be}^{7}$ and $\mathrm{C}^{11}$ from aluminum, have rising excitation functions.

Measurements of the formation cross sections of about 50 radioactive nuclides produced in the bom-

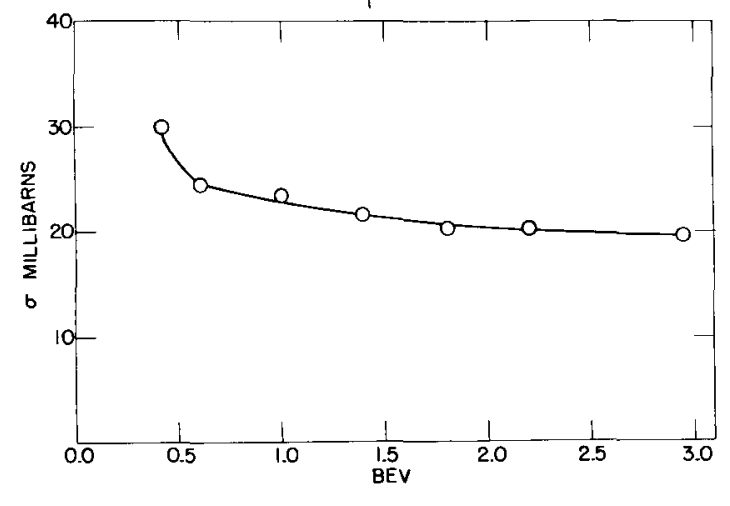

Figure 1. Excitation function of the $C^{12}(p, p n) C^{11}$ reaction.

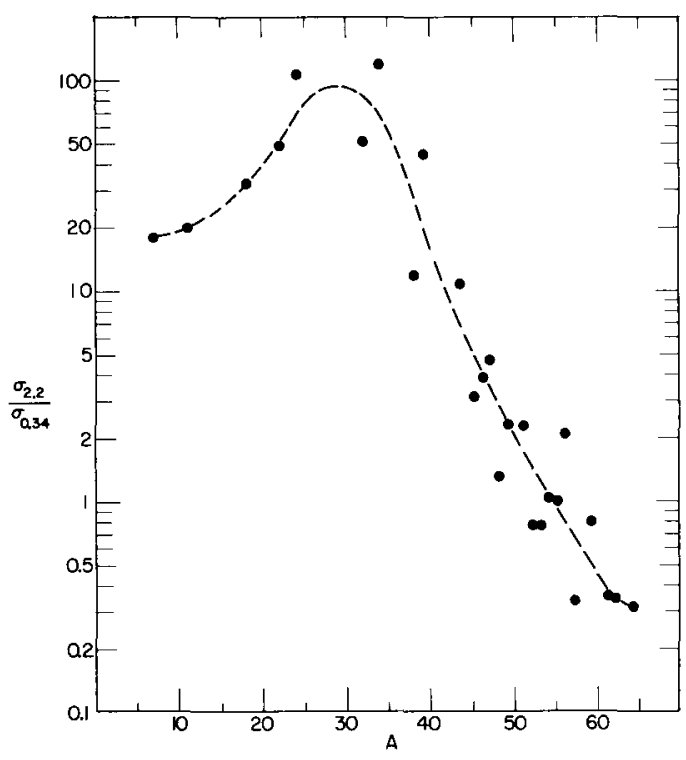

Figure 2. Ratios of formation cross sections at 2.2-Bev proton energy to those at $0.34 \mathrm{Bev}$ for copper spallation.

bardment of copper with 2.2-Bev protons are nearly complete. The striking difference between these results and those of a corresponding study at $340-\mathrm{Mev}$ proton energy (Batzel, Miller, and Seaborg, Phys. Rev. 84, 671 (1951)) are shown in Figure 2 in which the ratio of high energy to low energy cross section is plotted against product mass number. Deposition of small amounts of excitation energy, leading to products within a few mass numbers of the target, has become relatively less important at the higher energy, while loss of 15 or more nucleons is much more probable. In fact, interpolation of cross sections for undetected (stable or long-lived) product nuclides on the basis of approximate evaporation calculations leads to the conclusion that the total cross section for a given product mass number peaks somewhere in the region of $A=45$ to 50 .

The high probability of large energy transfers observed in this study (and also in other investigations mentioned below) cannot be accounted for by nucleon interactions alone. A plausible mechanism for imparting hundreds of Mev of excitation energy to a nucleus is the creation and subsequent scattering and reabsorption of a meson, all within the same nucleus. Experimental information on the energy spectra and interaction cross sections of $\pi$-mesons is not yet sufficiently complete to allow quantitative calculation, but existing data are qualitatively in ac- 
Table 1

Cross sections (in $\mathrm{mb}$ ) for the formation of light fragments from proton bombardments of lead

\begin{tabular}{lccccc}
\hline & \multicolumn{5}{c}{ Proton Energy (Bev) } \\
Product & 0.6 & 1.0 & 1.6 & 2.2 & \multicolumn{1}{c}{3.0} \\
\hline $\mathrm{F}^{18}$ & & $\sim 0.02$ & 0.18 & 0.48 & 0.60 \\
$\mathrm{Na}^{22}$ & & & & $<0.2$ & \\
$\mathrm{Na}^{24}$ & & 0.35 & 1.4 & 2.5 & 2.6 \\
$\mathrm{Mg}^{28}$ & $\sim 0.03$ & & 0.28 & 0.48 & 0.61 \\
$\mathrm{P}^{32}$ & 0.01 & 0.2 & 0.45 & 0.8 & 0.7 \\
\hline \hline
\end{tabular}

cord with the suggested mechanism. The fact that the cross sections for processes involving small energy transfers show a much smaller decrease with increasing energy for light elements than they do for copper may be accounted for by the larger escape probability of mesons from small nuclei. A more complete study of excitation functions of simple reactions in various mass regions is being planned.

Studies of the products of $2.2-\mathrm{Bev}$ proton bombardments of lead and bismuth have led to the conclusion that in this case, too, the most probable products are not in the immediate vicinity of the target, but perhaps some 20 mass numbers below. Because of the very large number of possible products, the information on the reactions of lead and bismuth is much less complete than that on the copper work. However, the formation cross sections of about two dozen nuclides which have been isolated show what appears to be a monotonic decrease with $A$ from a maximum near the upper end of the rare earths to about $A=60-70$. No pronounced fission peak exists in the yields- $v$ s-mass curve at this energy. Some products, e.g. Ba isotopes which at lower bombarding energies are formed by fission, have been shown to be spallation products in the 2.2-Bev bombardments. This was established by studies of the relative amounts and apparent ranges of these fragments leaving a target foil in the forward and backward directions. For a given product, fission gives rise to a much smaller forward-to-backward loss ratio than does spallation because the latter mechanism requires a greater transfer of excitation energy and therefore results in a larger forward center-of-mass motion.

A somewhat surprising result of the lead studies are the relatively high cross sections for the formation of light elements, listed in Table 1. The proc- esses leading to these products become prevalent only at energies above about $1 \mathrm{Bev}$, but even then the products are too abundant to be ascribed to conventional spallation reactions. They might represent products of a very asymmetric fission process or arise from an entirely different mechanism which might, for example, involve rupture of the nucleus into fragments before the distribution of excitation energy. Experiments on the ranges and angular distribution of the light fragments are now being planned to help solve these problems.

In order to gain a better understanding of the nuclear reactions observed at Bev energies and to establish possible correlations with meson processes, it is of interest to study the variation of cross sections with bombarding energy. A number of excitation functions have therefore been determined. The production of the $4.1-\mathrm{hr} \alpha$-emitter $\mathrm{Tb}^{149}$ from various heavy element targets lends itself well to this sort of study because the $\mathrm{Tb}^{149}$ can be measured directly in bombarded target foils after some shorter-lived a activities have decayed. Figure 3 shows the excitation functions for the production of $\mathrm{Tb}^{149}$ from $\mathrm{Ta}, \mathrm{Au}$, and $\mathrm{Bi}$. It is interesting to note that for each of the three reactions the threshold energy corresponds to $12 \mathrm{Mev}$ per emitted nucleon and the energy at the

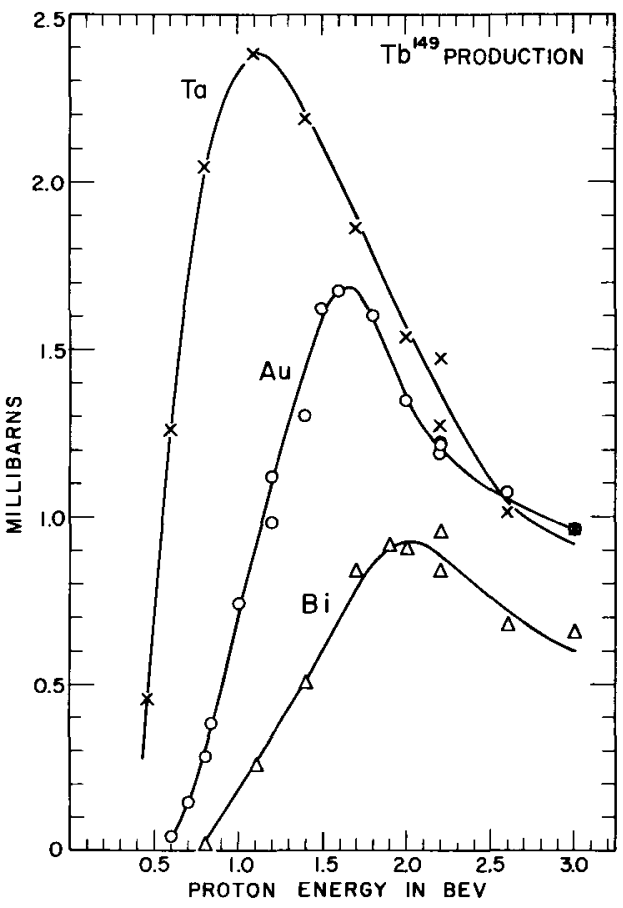

Figure 3. Excitation functions for the formation of the alpha emitter $\mathrm{Tb}^{149}$ from $\mathrm{Ta}, A u$, and $\mathrm{Bi}$. 
Table 2

Cross section (in $\mathrm{mb}$ ) for the formation of $\mathrm{Be}^{7}$

\begin{tabular}{lccccc}
\hline & \multicolumn{5}{c}{ Proton Energy (Bev) } \\
Target & 0.45 & 1.0 & 1.4 & 2.2 & 3.0 \\
\hline $\mathrm{C}$ & 14 & 13 & 13 & 13 & 13 \\
$\mathrm{Al}$ & 1.4 & 4.7 & 5.6 & 7.5 & 7.7 \\
$\mathrm{Cu}$ & 0.5 & 5.9 & & 10 & 13 \\
$\mathrm{Ag}$ & 0.1 & $\sim 3.5$ & & 12 & 13 \\
$\mathrm{Au}$ & 0.01 & $<2$ & & 6 & 9 \\
\hline
\end{tabular}

peak corresponds to about $33 \mathrm{Mev}$ per emitted nucleon. The formation of $\mathrm{Tb}^{149}$ from gold has been used to monitor high energy proton fluxes.

Other excitation function studies have dealt with the formation of $A^{3 i}$ and $A^{41}$ from copper, and this work will be extended to the production of argon and krypton isotopes from various other targets. Some data on the production of $\mathrm{Be}^{7}$ from several targets at a few energies are shown in Table 2. Values reported in the literature at $0.45 \mathrm{Bev}$ are included for comparison. The high cross sections for the formation of $\mathrm{Be}^{7}$ at 2 to $3 \mathrm{Bev}$ are consistent with photographic emulsion data on other light fragments such as $\mathrm{Li}^{8}$ and $\mathrm{B}^{8}$. It is clear that, at least in the case of the high $\mathrm{Z}$ targets $(\mathrm{Cu}, \mathrm{Ag}, \mathrm{Au}), \mathrm{Be}^{7}$ is formed not as the residue of many evaporations, but by some direct ejection mechanism. Studies on the production of other light fragments such as $\mathrm{H}^{3}$ and $\mathrm{C}^{11}$ are also in progress. The fact that energetic light fragments are formed in the interactions of $2.2-\mathrm{Bev}$ protons with copper was shown by the isolation and identification of a number of $\mathrm{Ga}, \mathrm{Ge}$, and $\mathrm{As}$ isotopes

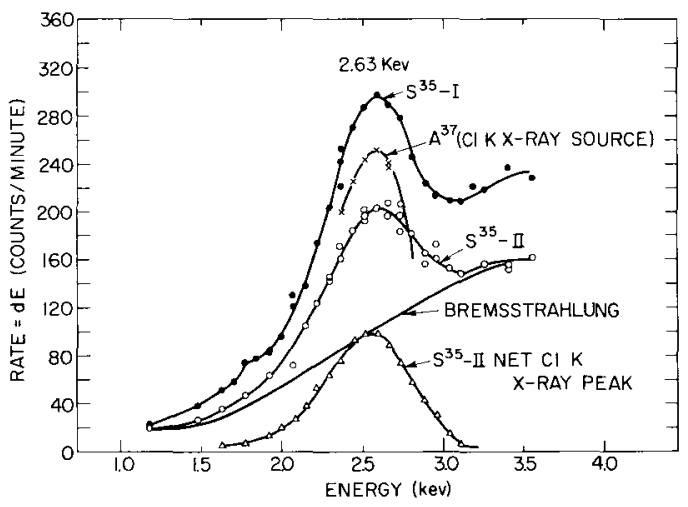

Figure 4. Pulse-height distribution representing photons emitted in the decay of $\mathrm{S}^{35}$. in irradiated copper foils. The formation of these nuclides is ascribed to secondary reactions of energetic $\mathrm{He}, \mathrm{Li}$, and $\mathrm{Be}$ nuclei with copper; with sufficiently thin targets, the apparent cross sections for making these trans-target nuclides decreased as would be expected for such secondary reactions.

The effects of nuclear transformation on the atomic electrons have been the subjects of study during the past year. Measurements have been made on the ejection of $\mathrm{K}$-shell electrons in the beta decay of $S^{35}$ and in the electron-capture decay of $A^{37}$. In the case of $\mathrm{S}^{35}$, the chlorine $\mathrm{K} \mathrm{x}$-rays and other photons were detected with a proportional counter and pulse-height analyzer; a magnetic field between source and counter served to bend away the beta rays. The observed pulse distribution from two $S^{35}$ sources are shown in Figure 4, together with the distribution from a standard source of $\mathrm{Cl} \mathrm{K} \mathrm{x}$-rays. Net $\mathrm{x}$-ray intensity from the $\mathrm{S}^{35}$, obtained after subtraction of a bremsstrahlung background from the observed curve, was compared with the beta-ray intensity. In $S^{35}$ the probability, per beta decay, of the removal of an electron from the $\mathrm{K}$ shell was found to be $3 \times 10^{-3}$, which is to be compared with the theoretical value of $4 \times 10^{-3}$. In the case of $\mathrm{A}^{37}$ the active gas was made part of the filling mixture of a small proportional counter, and the pulse-height distribution was measured with an analyzer. The intensity in

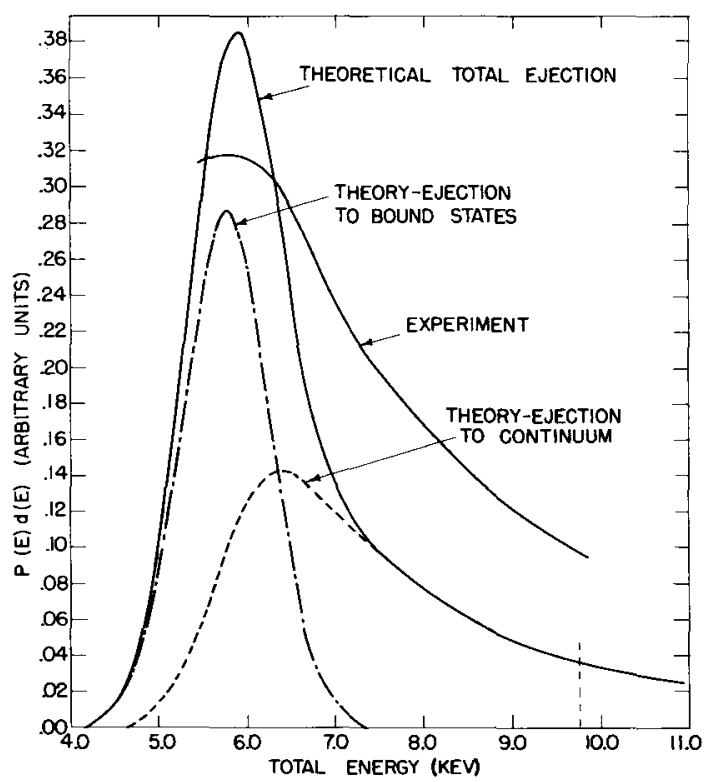

Figure 5. Pulse-height distributions representing ejection of the second $\mathrm{K}$ electron in the $\mathrm{K}$ capture decay of $\mathrm{A}^{37}$. 
the region of $2.8 \mathrm{kev}$, the $\mathrm{K}$ electron binding energy of chlorine, was compared with the intensity distribution in the energy interval from about $5.8 \mathrm{kev}$ to 9.8 $\mathrm{kev}$,corresponding to the ejection of a K electron with kinetic energy between zero and $4.0 \mathrm{kev}$. Seventythree percent of the theoretically predicted total effect should occur in this energy interval. The data, appropriately corrected, give a value of $3.9 \pm 0.7 \times 10^{-4}$ for the probability of double $K$ vacancy production per $\mathrm{K}$ capture in the experimental energy interval. The result is in agreement with the theoretical value (2 to 4) $\times 10^{-4}$. However, as shown in Figure 5, the promotion of the second $\mathrm{K}$ electron to bound states seems less probable than the theory predicts, while ejection to the continuum seems more probable. Work in this field is continuing.

Investigation of the modes of decay of electroncapture isotopes and of some fission products yielded results of interest during the year. The disintegration of 13 -day $\mathrm{I}^{126}$, which proceeds partially by electroncapture and positron emission to $\mathrm{Te}^{126}$ and partially by negative beta emission to $\mathrm{Xe}^{126}$, was studied with the gray wedge scintillation coincidence spectrometer developed by the Electronics Division. The information obtained, combined with that obtained in an earlier study in this Department, establishes the decay scheme and level assignments shown in Figure 6. It is noteworthy that the experimentally determined

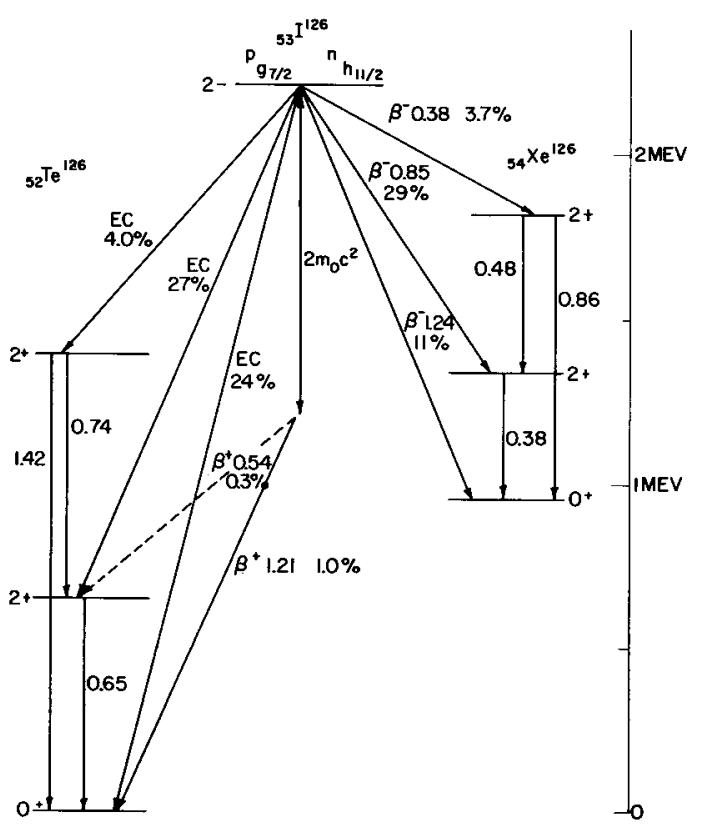

Figure 6. Decay scheme of $\mathrm{I}^{226}$.

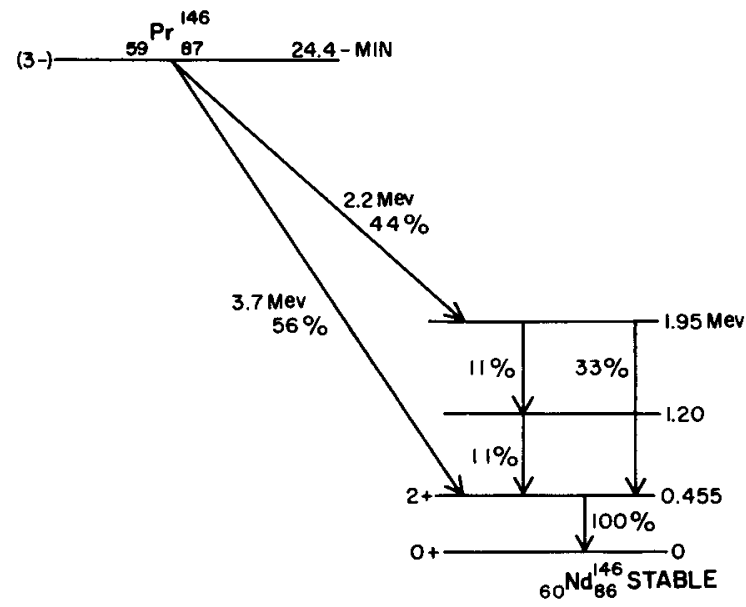

Figure 7. Decay scheme of $\operatorname{Pr}^{146}$.

ratio, $\operatorname{Prob}_{\text {Kcapt }} / \operatorname{Prob}_{\beta}{ }^{+}$for the transition to the ground state of $\mathrm{Te}^{126}$, represents perhaps the only instance in which this quantity has been measured for a first forbidden transition of the type $\Delta I=2$ (with parity change) with accuracy sufficient for comparison with theory. The result agrees with the theoretical ratio calculated for "tensor" interaction.

The cerium and praseodymium members of two chains produced in the fission of uranium were studied in the course of the year. Some work on one of these, the mass 146 chain, was mentioned in the

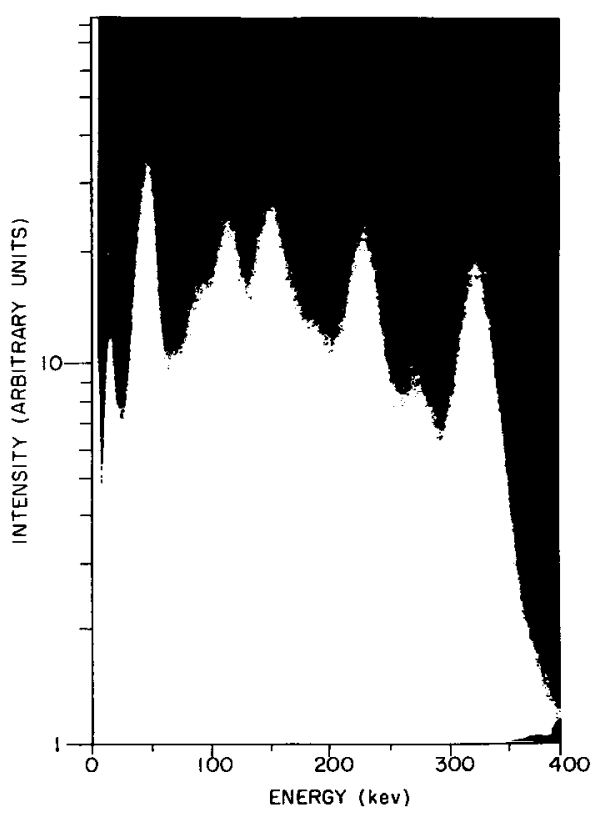

Figure 8. Grey wedge photograph of the $\mathrm{Ce}^{146}$ gamma-ray spectrum. 
last annual report (BNL 246 (AS-7)). The new information about the radiations of $24.4-\mathrm{min} \operatorname{Pr}^{146}$, obtained by means of the gray wedge coincidence spectrometer, supports the decay scheme diagramed in Figure 7. The 13.9-min $\mathrm{Ce}^{1+6}$ parent of the $\operatorname{Pr}^{146}$ was found to emit beta rays of $700-\mathrm{kev}$ maximum energy in coincidence with all the observed gamma rays. A gray wedge photograph of the gamma spectrum is shown in Figure 8. Analysis of this spectrum and of coincidence spectra yield the information presented in Table 3. A new 3.0-min Ce fission product has been identified in Ce fractions rapidly separated from neutron-irradiated uranium. It was found that this isotope decays to a 5.95-hr Pr daughter by the emission of a $2.0-\mathrm{Mev}$ beta spectrum. The daughter decays by emission of beta rays of $1.7-\mathrm{Mev}$ maximum energy; gamma rays seem to be absent. A comparison of the observed decay energies of this Ce-Pr pair with those calculated for various mass numbers from a semi-empirical mass formula leads to the tentative assignment of the pair to mass number 145 .

Experiments are being made in an effort to detect neutrino capture by nuclei, a process approximately the inverse of electron capture. The particular reaction involved in these experiments is $\mathrm{Cl}^{37}\left(v, \mathrm{e}^{-}\right) \mathrm{A}^{37}$. Carbon tetrachloride is irradiated for about one month at the reactor in the neutrino, or anti-neutrino, flux provided by the decaying fission products. The 35-day $A^{3 \pi}$ activity is removed by helium sweep and, after purification, is introduced into a small low level G-M counter for measurement. Preliminary results obtained with 206 liters of carbon tetrachloride established an upper limit of $2 \times 10^{-42} \mathrm{~cm}^{2}$ for the reaction cross section if neutrinos and anti-neutrinos are the same. Work is now being done with 3900 liters

Table 3

Gamma rays observed from $13.9-\mathrm{min} \mathrm{Ce}^{1+6}$

\begin{tabular}{lll}
\hline $\begin{array}{c}\text { Selecting } \\
\gamma \text {-ray (kev) }\end{array}$ & $\begin{array}{c}\text { Relative intensity } \\
\text { (including efficiency } \\
\text { corrections) }\end{array}$ & $\begin{array}{c}\gamma \text { rays and K x-rays } \\
\text { observed in } \\
\text { coincidence" spectrum } \\
\text { (kev) }\end{array}$ \\
\hline 320 & 1.00 & (Nothing) \\
220 & $0.52 \pm 0.10$ & $36,110,142$ \\
142 & $0.42 \pm 0.08$ & 36,220 \\
110 & $0.20 \pm 0.05$ & $36,220,250$ \\
270 & $0.12 \pm 0.03$ & 36,50 \\
250 & low & 36,110 \\
50 & low & 270 \\
36 (x-ray) & high & $36,110,142,220,270$ \\
\hline
\end{tabular}

of liquid to achieve higher sensitivity. In a first experiment with this quantity, $A^{37}$ was produced by cosmic radiation in an amount 30 times the minimum detectable. It has been found that $20 \mathrm{ft}$ of earth lowers the cosmic-ray production rate of $A^{37}$ to the extent that it about equals the production rate expected from the Hanford reactor neutrino flux if the theoretical cross section of $2 \times 10^{-45} \mathrm{~cm}^{2}$ is correct.

\section{CHEMISTRY AND APPLICATIONS OF ISOTOPES}

A detailed thermodynamic and statistical mechanical analysis has been made of the exchange of deuterium between solid uranium hydride and hydrogen gas. This exchange was discovered several years ago in this laboratory. A plot of the equilibrium constant for the reaction

$$
\mathrm{UH}_{3}(\mathrm{~s})+\mathrm{HD}(\mathrm{g})=\mathrm{UH}_{2} \mathrm{D}(\mathrm{s})+\mathrm{H}_{2}(\mathrm{~g})
$$

as a function of temperature is given in Figure 9. The entire curve can be understood in terms of the following: (1) the zero point energy difference of $\mathrm{UH}_{3}$ and $\mathrm{UH}_{2} \mathrm{D}$ is greater than that of $\mathrm{H}_{2}$ and $\mathrm{HD}$; (2) the heat of reaction is small even for an isotope exchange reaction; (3) the entropy change in the reaction is significant; (4) the optical vibration frequencies in $\mathrm{UH}_{3}$ are smaller than the stretching frequency in hydrogen. A calculation of the entropy change in the reaction based on the structure of $\mathrm{UH}_{3}$ is in quantitative agreement with experiment. The difference in the heat of formation of $\mathrm{UH}_{3}$ and $\mathrm{UD}_{3}$ calculated from the exchange data and thermochemical cycles is in excellent agreement with the calorimetric determinations of Abraham and Flotow at the Argonne National Laboratory. The minimum in

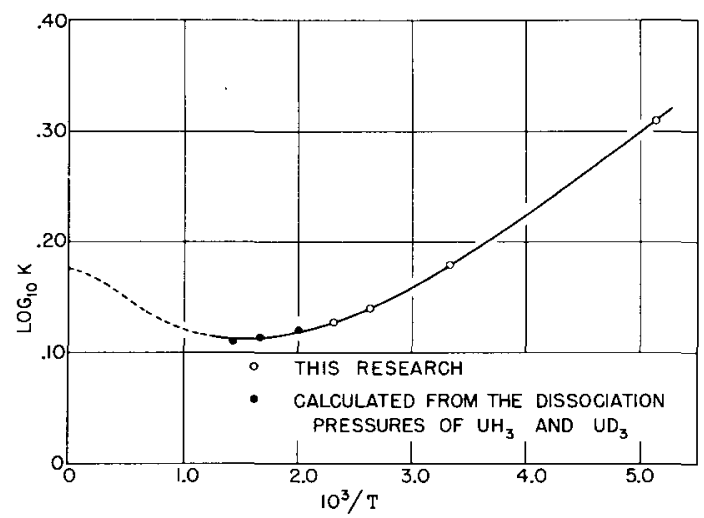

Figure 9. Equilibrium plot of $\log \mathrm{K}$ vs. $1 / \mathrm{T}$ for the reaction $\mathrm{UH}_{3}(\mathrm{~s})+\mathrm{HD}(\mathrm{g})=\mathrm{UH}_{2} \mathrm{D}(\mathrm{s})+\mathrm{H}_{2}(\mathrm{~g})$. 
the plot of $\log K l^{\prime}, 1 / \mathrm{T}$ is explained by the reasonable assumption of 2 frequencies per $\mathrm{UH}_{3}$ unit in the solid in the range from 400 to $1500 \mathrm{~cm}^{-1}$. This also explains the fact that the ratio of the dissocia. tion pressures of $\mathrm{UH}_{3}$ and $\mathrm{UD}_{3}, 0.70$, is independent of temperature in the range of 530 to $700^{\circ} \mathrm{K}$.

A study of the effect of substitution of tritium and deuterium for protium on the rate of the unimolecular isomerization of cyclopropane to propylene has been undertaken. This study should shed light on some detailed theoretical calculations recently carried out by N. B. Slater for this reaction, as well as the Lindemann hypothesis for the fall-off of the rate constant of unimolecular reactions at low pressures. At $500^{\circ} \mathrm{C}$ and 1 atmosphere, $k_{r} / k_{H}$ is found to be $0.89 \pm .02$ for cyclopropane containing 1 tritium atom per molecule. This is consistent with predictions based on an activated state in which there is a hydrogen atom migration rather than C-C bond rupture. The experiments are being carried to lower pressures, and the analogous reaction with $C_{3} D_{6}$ is being studied.

The mass spectrometer has been used to identify fragments produced in the electron bombardment of lithium iodide vapor, in equilibrium with the condensed phases, and to study their behavior as a function of temperature. A plot of the intensity of the $\mathrm{Li}^{+}$ and $\mathrm{Li}_{2} \mathrm{I}^{+}$ions measured in the mass spectrometer as a function of the temperature of the condensed phase is given in Figure 10. Ions derived from Lil, $\mathrm{Li}_{2} \mathrm{I}_{2}$, and $\mathrm{Li}_{3} \mathrm{I}_{3}$ molecules in the gas phase are definitely identified. At $0^{\circ} \mathrm{K}$ the heat of sublimation of monomer and dimer is $44.5 \pm 7$ and $45.0 \pm 2 \mathrm{kcal} \mathrm{mol}^{-1}$, respectively. At the same temperature the heat of dissociation of the gaseous dimer to monomer is $44.0 \pm 2.2 \mathrm{kcal}$. The heat of sublimation of the monomer leads to a value of $173.3 \pm 3 \mathrm{kcal} \mathrm{mol}^{-1}$ for the lattice energy of Lil. This may be compared with the theoretical value of $174.1 \mathrm{kcal} \mathrm{mol}^{-1}$ calculated by Mayer and Helmholz.

As an aid in the study of the effect of isotopic substitution on electron impact, a new ion source has been developed and constructed for use in a $60^{\circ}$ mass spectrometer. The essential features of the new source are: (1) The ionization region is almost entirely enclosed, which reduces the contribution to the mass spectrum from products resulting from thermal reactions at or near the filament. In addition background peaks are considerably repressed. (2) An increase in the magnetic field in the source pro-

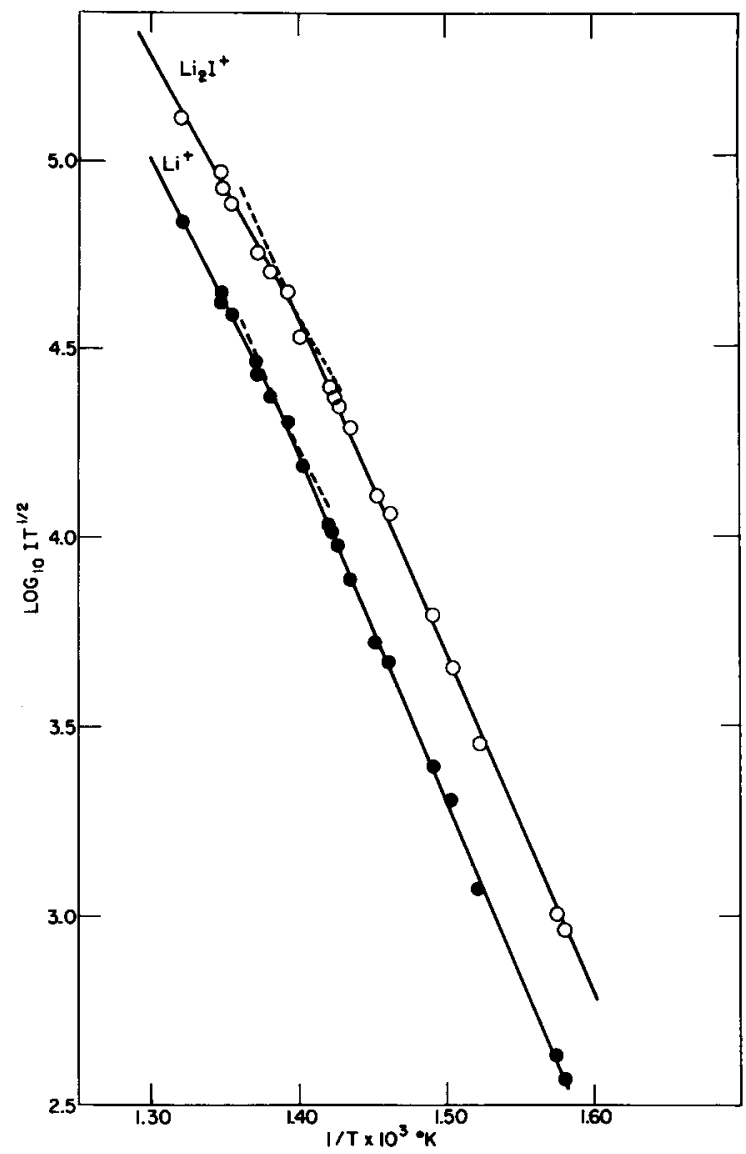

Figure 10. Intensity of $\mathrm{Li}^{+}$and $\mathrm{Li}_{2} \mathrm{I}^{+}$as a function of temperature. The break in the lines is at the melting point.

vides better electron collimation. This permits the use of higher repeller voltages and results in higher ion collection efficiencies and smaller discrimination.

This new source has been used to study the effect of $\mathrm{O}^{18}$ on bond rupture in $\mathrm{O}_{2}$ and both $\mathrm{C}^{13}$ and $\mathrm{O}^{18}$ on rupture in $\mathrm{CO}_{2}$. In the case of the $\mathrm{O}_{2}$ experiments, a significant correction to the mass spectrometer peak at mass 18 had to be applied for water vapor swept into the source from the walls by the gas sample. The results obtained as a function of electron voltage are given in Table 4. The theoretical values are calculated by the use of the Franck-Condon principle, and the mass spectrum of $\mathrm{O}^{16} \mathrm{O}^{16}$ is utilized to evaluate the critical internuclear distance for transitions which give rise to bond rupture. The agreement between the calculated and observed values is reasonable. In the experiments with 20-volt electrons, the discrepancy may be attributed to uncertainties in the correction for $\mathrm{H}_{2} \mathrm{O}^{16_{+}}$, which are more significant at the low electron energies than at higher ones. 
Table 4

Relative change in pattern for $\mathrm{O}^{18}$ substituted oxygen

\begin{tabular}{ccc}
$\begin{array}{c}\text { Nominal electron } \\
\text { energy (volts) }\end{array}$ & $\begin{array}{c}2\left(\mathrm{O}^{182} / \Sigma \mathrm{O}^{16} \mathrm{O}^{18}\right) \\
\text { (observed) }\end{array}$ & $\begin{array}{c}\left(\mathrm{O}^{16+} / \mathrm{\Sigma O}^{16} \mathrm{O}^{16}\right. \\
\text { (calculated) }\end{array}$ \\
\hline 20 & 0.88 & 0.930 \\
25 & 0.98 & 0.948 \\
35 & 0.97 & 0.959 \\
\hline
\end{tabular}

The results obtained with $\mathrm{C}^{13}$ and $\mathrm{O}^{18}$ substitution in $\mathrm{CO}_{2}$ on the relative abundance of $\mathrm{C}^{+}$and $\mathrm{CO}^{+}$ ions produced by electron impact are given in Table 5. The experimental data on $\mathrm{C}^{13} \mathrm{O}_{2}{ }^{16}$ are in good agreement with some of the work from other laboratories, but not with all the published data. The discrepancies are believed to be mainly due to differences in the experimental details. In this respect, it is believed that the new source used in the present experiments leads to more reliable results than have been obtained previously. The theoretical calculations again are based on the Franck-Condon principle, and, as in the case of the diatomic molecule, they utilize the data on $\mathrm{C}^{12} \mathrm{O}_{2}{ }^{16}$ to evaluate the atomic configurations which result in bond rupture. The agreement with experiment is within the experimental error. It is significant to note that the extension of the theory to the polyatomic molecule, $\mathrm{CO}_{2}$, which considers all the vibrations in the molecule, predicts larger fragmentation of the heavy ion than the light one in some cases, which is in agreement with experiment. This results from the fact that the positions of the atoms along the internuclear axis are determined by both the symmetric and antisymmetric stretching coordinates.

In an investigation of the relative abundance of the chlorine isotopes, $\mathrm{Cl}^{35} / \mathrm{C}^{37}$, no difference, within 0.2 percent, is found between chlorine of marine,
Table 6

Relative abundance of chlorine isotopes

\begin{tabular}{lll}
\hline \multicolumn{1}{c}{ Investigator } & Year & $\mathrm{Cl}^{35} / \mathrm{Cl}^{37}$ \\
\hline Aston & 1919 & $3.0-3.1$ \\
Kallman and Lazereff & 1933 & 3.24 \\
Nier & 1936 & $3.07 \pm .03$ \\
Harkins and Stone & 1926 & 3.096 \\
This Research & 1954 & $3.13 \pm .03$ \\
\hline \hline
\end{tabular}

igneous, and sedimentary origin. Samples containing different amounts of chloride were analyzed. The chlorine was introduced into the mass spectrometer in the form of methyl chloride. Chemical fractionation of the chlorine isotopes between these sources is expected to be of the order of a few tenths of a percent. This would indicate no fractionation of the chlorine isotopes in these samples from nuclear processes. A comparison with previous determinations is given in Table 6. The measurements of Harkins and Stone are from the chemical determination of the atomic weight of chlorine by the silver chloride method. The abundances are calculated by the use of Nier's determination (1951) of the masses of the chlorine isotopes. The chemical determination, which is the most precise one in the table, is bracketed by the mass spectrometer determination.

\section{CHEMICAL EFFECTS OF RADIATION}

\section{Aqueous Solutions}

The modes of chemical decomposition of water by gamma rays, which underlie the variety of radiation-chemical effects observed in aqueous solutions of all kinds, have been considerably clarified in detail during the year. Radiation decomposes water into the molecules $\mathrm{H}_{2}$ and $\mathrm{H}_{2} \mathrm{O}$ and the free radicals $\mathrm{H}$ and

Table 5

Relative change in pattern for $\mathrm{C}^{13}$ and $\mathrm{O}^{18}$ substitution in $\mathrm{CO}_{2}$

\begin{tabular}{|c|c|c|c|c|}
\hline & \multicolumn{2}{|c|}{ Comparison of $\mathrm{C}^{13} \mathrm{O}_{2}^{16}$ and $\mathrm{C}^{12} \mathrm{O}_{2}^{16}$} & \multicolumn{2}{|c|}{ Comparison of $\mathrm{C}^{12} \mathrm{O}^{16} \mathrm{O}^{18}$ and $\mathrm{C}^{12} \mathrm{O}_{2}^{16}$} \\
\hline & $\left(\mathrm{C}^{13} \mathrm{O}^{16+} / \mathrm{C}^{13} \mathrm{O}_{2}^{16+}\right)$ & $\left(\underline{\mathrm{C}^{13+} / \mathrm{C}^{13} \mathrm{O}_{2}^{16+}}\right)$ & $\left(\mathrm{C}^{12} \mathrm{O}^{18+} / \mathrm{C}^{12} \mathrm{O}^{16} \mathrm{O}^{18+}\right)$ & $\left(\underline{\mathrm{C}^{12+} / \mathrm{C}^{12} \mathrm{O}^{16} \mathrm{O}^{18+}}\right)$ \\
\hline & $\overline{\left(\mathrm{C}^{12} \mathrm{O}^{16+} / \mathrm{C}^{12} \mathrm{O}_{2}^{16+}\right)}$ & $\left(\overline{\mathrm{C}^{12+} / \mathrm{C}^{12} \mathrm{O}_{2}^{16+}}\right)$ & $\overline{\left(\mathrm{C}^{12} \mathrm{O}^{16+} / \mathrm{C}^{12} \mathrm{O}_{2}^{16+}\right)}$ & $\overline{\left(\mathrm{C}^{12+} / \mathrm{C}^{12} \mathrm{O}_{2}^{16+}\right)}$ \\
\hline Experimental & .974 & 1.022 & 1.01 & .98 \\
\hline Theoretical & .98 & 1.011 & 1.001 & .97 \\
\hline
\end{tabular}


$\mathrm{OH}$, the radicals being unobservable directly and their existence and number inferred from the chemical changes undergone by added solutes. Since the radicals can also react with the molecular $\mathrm{H}_{2}$ and $\mathrm{H}_{2} \mathrm{O}_{2}$ formed from the water, determination of the initially produced amounts of the four products is a complex problem, involving determination and correlation of the yields of all the chemical substances formed on irradiation of a variety of solutions under a range of conditions.

The gamma-ray yield of molecular hydrogen from water has now been definitely established as 0.45 molecules $\mathrm{H}_{2}$ per 100-ev energy input, independent of the nature and concentration of added solutes up to at least $0.003 \mathrm{M}$ and practically independent of temperature from $4^{\circ}$ to $65^{\circ} \mathrm{C}$. This result has been confirmed at Oak Ridge. The hydrogen yield is unchanged in $0.4 \mathrm{M} \mathrm{NaCl}$, but it is lowered by 13 percent in $0.4 \mathrm{M} \mathrm{H}_{2} \mathrm{SO}_{4}$ and by 55 percent in $1 \mathrm{MCuSO}_{4}$.

The yield of molecular $\mathrm{H}_{2} \mathrm{O}_{2}$ is definitely somewhat higher than that of $\mathrm{H}_{2}$. The $\mathrm{H}_{2} \mathrm{O}_{2}$ yield, unlike that of $\mathrm{H}_{2}$, is appreciably reduced by addition of certain solutes (such as $\mathrm{NO}_{2}^{-}$and $\mathrm{Br}^{-}$) at concentrations well below $0.001 M$. There is no indication that the $\mathrm{H}_{2} \mathrm{O}_{2}$ yield ever falls below that of $\mathrm{H}_{2}$. To the excess of $\mathrm{H}_{2} \mathrm{O}_{2}$ over $\mathrm{H}_{2}$ must correspond, by material balance, an excess of $\mathrm{H}$ atoms over $\mathrm{OH}$ radicals. Since these excess $\mathrm{H}$ atoms tend to reduce $\mathrm{H}_{2} \mathrm{O}_{2}$ to water, a value of the true initial yield of $\mathrm{H}_{2} \mathrm{O}_{2}$ is hard to obtain. The above conclusions are obtained largely from a continuation of the study of $\mathrm{KNO}_{2}$ solutions described in the last annual report.

The kinetics of nitrate and $\mathrm{H}_{2} \mathrm{O}_{2}$ formation were studied in irradiated solutions of $\mathrm{KNO}_{2}$ containing oxygen. Interpretation of the results served to eliminate some alternative mechanisms for the reaction in the oxygen-free system and thus to define the mechanism in that case. Knowing the mechanism, it then became possible to extrapolate the peroxide yield in the de-aerated nitrite solutions back to its true initial value. The results are given in Table 7. The peroxide yield decreases significantly with increasing nitrite concentration and at higher concentrations appears to level off at a value slightly above the hydrogen yield. Data recently obtained at Oak Ridge on aerated bromide solutions, which have now been verified at BNL (Table 7), indicate that bromide ion has a similar effect in depressing the primary peroxide yield, but that the amount of bro-
Table 7

Variation with solute concentration of direct molecular $\mathrm{H}_{2} \mathrm{O}_{2}$ production from water by gamma rays

\begin{tabular}{|c|c|c|c|}
\hline \multirow{2}{*}{$\begin{array}{l}\text { Conc. of } \\
\mathrm{NO}^{-} \text {or } \\
\mathrm{Br}^{-}(M)\end{array}$} & \multicolumn{3}{|c|}{ Calculated primary $\mathrm{H}_{2} \mathrm{O}_{2}$ (molecules per $100 \mathrm{ev}$} \\
\hline & $\begin{array}{l}\mathrm{NO}_{2}^{-} \\
\text {neutral }\end{array}$ & $\begin{array}{c}\mathrm{Br}^{-} \mathrm{O}_{.,} \\
0.01 N \mathrm{H}_{2} \mathrm{SO}_{4} \\
\text { (Brookhaven) }\end{array}$ & $\begin{array}{c}\mathrm{Br}^{-} \mathrm{O}_{3} \\
\text { 0 olN } \mathrm{H}_{2} \mathrm{SO}_{+} \\
\text {(Oak Ridge) }\end{array}$ \\
\hline $10^{-5}$ & - & - & 0.75 \\
\hline $10^{-4}$ & 0.66 & 0.73 & 0.72 \\
\hline $5 \times 10^{-4}$ & 0.55 & 0.70 & - \\
\hline $10^{-3}$ & 0.52 & - & 0.68 \\
\hline $6 \times 10^{-3}$ & 0.55 & 0.64 & - \\
\hline $10^{-2}$ & - & - & 0.58 \\
\hline
\end{tabular}

mide required for a given lowering is several times the amount of nitrite required. Studies of the effects of other solutes are in progress.

The molecular products are believed to originate in the densely ionized "hot spots" formed by the tracks of slow secondary electrons, and to arise by a process of initial recombination of like radicals formed by the dissociation of water molecules lying close together. The effect of dilute reducing agents on the peroxide yield may mean that the process of recombination extends out to greater distances from the site of formation than heretofore thought possible. If the speed of the primary particle is decreased (by changing the quality of the applied radiation), the "hot spots" lie closer together and eventually coalesce, which thus gives an increased yield of molecular products. A precise determination of these yields as a function of particle speed may be expected to further clarify the situation. The best source of particles having speeds in the range of interest is the cyclotron. Such a program is getting under way with the Brookhaven cyclotron.

The ferrous sulfate oxidation by gamma rays in aerated solution has been the subject of many papers, and a comprehensive and precise study of this reaction was undertaken to clear up reported discrepancies. The yield was found to be essentially independent of temperature. The amount of reaction increases linearly with dose at high $\mathrm{H}_{2} \mathrm{SO}_{4}$ concentrations, but at lower acidities the product, ferric iron, exerts an inhibiting effect. The initial rate decreases only slightly with decreasing acidity, and this small decrease is ascribed to a decrease in the total number of free radicals formed by the radiation. The kinetics follow in detail those expected 
from J. Weiss' mechanism, if proper account is taken of the molecular hydrogen yield. A similar study is now under way on the same solutions irradiated in the absence of air.

The irradiation of benzoic acid in solution is known to lead to hydroxybenzoic acids, and on the assumption of a free radical mechanism it has been supposed that $\left(\mathrm{C}_{6} \mathrm{H}_{4} \mathrm{COOH}\right)_{2}$ should be found. On long irradiation of acid benzoate solutions, however, an insoluble white precipitate formed instead. When $\mathrm{HCl}$ was present during irradiation, the precipitate contained chlorine, as expected, since $\mathrm{OH}$ radicals have long been thought to react with $\mathrm{H}^{+}+\mathrm{Cl}^{-}$to give $\mathrm{Cl}$ atoms.

A study was made of the kinetics of the reaction between benzoic acid and ferric ion in solution activated by ultraviolet light, which has been supposed to occur through free $\mathrm{OH}$ radicals. The photactivated ferric ion $\mathrm{Fe}(\mathrm{OH})^{++}$was supposed by Evans and Uri to form ferrous ion and free $\mathrm{OH}$, which could react with benzoic acid or other organic substrate added, or alternatively with ferrous ion as the latter accumulated (or was intentionally added). Their data, however, could be fit just as well by a mechanism in which the photoactivated complex reacts directly with the substrate or ferrous ion without dissociation. A distinction could be made between the two mechanisms by a series of experiments in which the total concentrations of ferrous ion and benzoic acid were varied while their ratio was kept constant. The experiments were carried out and led to the conclusion that the free radical mechanism is in fact the correct one.

Experiments on thermal and photochemical reactions of ceric salts are being carried out with the aim of testing the free radical theory for this case also.

\section{Dosimetry}

The use of ferrous sulfate solution as a standard for gamma-ray and hard $\mathbf{x}$-ray dosimetry has become almost universal among radiation chemists. The absolute value of the ferrous sulfate yield, however, has an importance beyond radiation chemistry, in that it provides the only practical check on the absolute accuracy of the standard cavity ion chamber method of gamma-ray dosimetry. Conversion of ion chamber readings to dose delivered to any material (defined as energy put into the material per unit mass) depends among other things on knowledge of the energy, W, required to form an ion pair in air. W is usually taken as $32.5 \mathrm{ev}$ for gamma rays, but this value is actually uncertain by several percent. By proper comparison of ion chamber readings with the amount of ferrous sulfate oxidized in a given radiation field, the value of $W$ can be ascertained, if the absolute value of the ferrous sulfate yield is known.

During the year, determination of the absolute value of the ferrous sulfate yield was completed and published. Cathode rays at 1 and $2 \mathrm{Mev}$ were the radiation source, and the absolute energy input was obtained from measurements of the cathode-ray current and voltage, with small corrections required for back-scattering, window absorption, and bremsstrahlung. The yield was found to be independent of radiation intensity over a wide range. The result was in very good agreement with the latest calorimetric values obtained elsewhere, but the accuracy was not much better than \pm 3 percent. An ion chamber comparison, carried out with hard $\mathbf{x}$-rays in this laboratory by members of the Health Physics and Electronics Divisions, indicated that $W$ was a few percent higher than the accepted value, but this conclusion is uncertain because of the errors in both determinations. It is at least clear now that the results reported in the literature which give a ferrous sulfate yield 30 percent higher than those obtained in this laboratory were in error, either because of subtle flaws in ion-chamber design or because of organic contamination of the ferrous sulfate solutions.

Preparations are now under way for better determinations of both the cathode-ray yield and the ion chamber comparison. In this connection, measurements of the back-scattering of electrons of 1 and 2 Mev have been made for targets of very low atomic number ( $\mathrm{Li}, \mathrm{Be}, \mathrm{C}, \mathrm{Mg}$ ). The results show that the back-scattering does not increase linearly with $\mathrm{Z}$ in this region, as formerly thought, and the back-scattering correction from aqueous solutions is smaller than previously thought by about 1 percent of the total beam.

\section{Organic Liquids}

A basic general problem in the radiation chemistry of solid or liquid matter is that of specifying the mechanisms whereby energy is transferred from the point where it first appears in the material to the point where it produces an observed effect. Possible mechanisms are diffusion of free radicals, capture of free electrons, and intermolecular transfer of electronic excitation and of positive electric charge. 
Studies of solutions of various polar compounds in hydrocarbons have been made which show that there are at least two modes of energy transfer from solvent to solute molecule which may be quantitatively characterized: one appears to involve attack on the solute by free radicals, and the second is to be identified with one or more of the three other processes mentioned above.

On irradiation of solutions of iodine in hydrocarbons, the iodine disappears, but if present at concentrations of less than $10^{-4}$ mole fraction, the hydrogen yield from decompostion of the solvent is unaffected. When the mole fraction is increased to $10^{-2}$, however, the hydrogen yield drops from 5 to 2 molecules per $100 \mathrm{ev}$. Methyl iodide (Figure 11), ethyl iodide, and carbon tetrachloride have very similar effects on the hydrogen yield in this concentration range. The disappearance of $I_{2}$ at very low concentrations is assumed to be due to the reaction of free radicals formed from the solvent hydrocarbon; since the yield of hydrogen gas is unchanged by the iodine, the hydrogen is formed by a process not involving radicals. This process, however, is interfered with by concentrations of $I_{2}$, methyl iodide, etc, above $10^{-4}$ mole fraction. The interference may involve electron capture by the added halides, or possibly excitation transfer processes.

It has been shown in three ways that the reaction which quenches $\mathrm{H}_{2}$ formation also produces dissociation of the added halide to free radicals and halogen

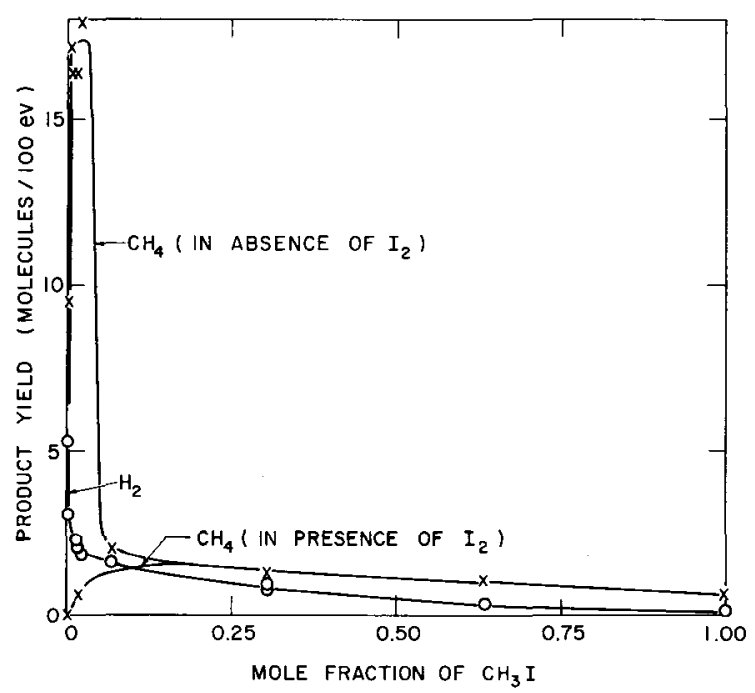

Figure 11. Hydrogen and methane yields in the gamma radiolysis of solutions of methyl iodide in cyclohexane. atoms: (1) If a very low concentration of radioactive $I_{2}$ is added along with the alkyl halide, the corresponding radioactive alkyl iodide is produced and may be isolated by carrier separation methods. This must arise by pickup of the free alkyl radical by the tagged $\mathrm{I}_{2}$. (2) An extremely high methane yield is found in solutions of 1 to 5 mole $\% \mathrm{CH}_{3} \mathrm{I}$ in cyclohexane (Figure 11). Addition of $10^{-3}$ to $10^{-6}$ mole fraction of $I_{2}$ prevents the formation of most of the methane. Here in the absence of $I_{2}$ the free methyl arising from methyl iodide dissociation can start a methane-producing chain:

$$
\mathrm{CH}_{3} \cdot+\mathrm{RH}=\mathrm{CH}_{4}+\mathrm{R} \cdot ; \mathrm{R} \cdot+\mathrm{CH}_{3} \mathrm{I}=\mathrm{RI}+\mathrm{CH}_{3} \text {. }
$$

Below $5 \% \mathrm{CH}_{3} \mathrm{I}$, the iodine from the $\mathrm{CH}_{3} \mathrm{I}$ dissociation is destroyed by radicals coming from the radical mode of dissociation of the solvent. Above $5 \%$, however, so much $\mathrm{CH}_{3} \mathrm{I}$ is being dissociated that the solvent-produced radicals are no longer sufficient to destroy it all. The iodine reacts with the chain-carrying radicals and breaks the chain, so that the methane formation drops abruptly above 5 percent $\mathrm{CH}_{3} \mathbf{I}$. (3) When $7 \times 10^{-3}$ mole fraction of $\mathrm{HI}$ was added to cyclohexane, the $\mathrm{H}_{2}$ yield increased from 5.3 molecules per $100 \mathrm{ev}$ (for the pure solvent) to 7 . The additional $\mathrm{H}_{2}$ comes from the action on the solvent of $\mathrm{H}$ atoms formed by dissociation of the HI. Addition of $3 \times 10^{-4}$ mole fraction of $\mathrm{I}_{2}$ along with the $\mathrm{HI}$ reduced the $\mathrm{H}_{2}$ yield below 5 , which shows that low concentrations of $I_{2}$ are sufficient to scavenge free $\mathrm{H}$ atoms in a hydrocarbon medium and thus provides further evidence that the $\mathrm{H}_{2}$ from the hydrocarbon decomposition does not arise from a process involving free radicals or $\mathrm{H}$ atoms.

\section{CHEMICAL EFFECTS OF NUCLEAR TRANSFORMATIONS}

Research on various aspects of the chemical results of nuclear transformations in the several states of matter has been pursued, mainly with the view to a better understanding of the nature of recoil processes and, in part, to the potential utility of such processes in the preparation of isotopically labeled materials.

A study of the beta decay of $\mathrm{C}^{14}$ in ethane- $\mathrm{C}_{2}{ }^{14}$ as a gas has been completed. The purpose of this research was to see whether a covalent chemical bond could survive a beta transformation of one of the bound nuclei. The over-all "yield" of the following reaction, namely, from ethane to methylamine, 


$$
\begin{aligned}
\mathrm{C}^{14} \mathrm{H}_{3}-\mathrm{C}^{14} \mathrm{H}_{33} & \rightarrow\left[\mathrm{C}^{14} \mathrm{H}_{3}-\mathrm{N}^{1 \cdot 4} \mathrm{H}_{3}\right]+\beta \\
& \rightarrow \mathrm{C}^{14} \mathrm{H}_{3}-\mathrm{NH}_{2}+\mathrm{H}^{+}
\end{aligned}
$$

is demonstrably $50 \pm 5 \%$. Clearly, a nuclear transformation takes place without bond rupture in this case, where the maximum recoil energy of the $C^{14}$ nucleus is about $6 \mathrm{ev}$ and the average recoil energy about $3 \mathrm{ev}$. The bond survives not only this recoil energy excitation but several other disruptive effects, such as the sudden change in $\mathrm{Z}$ and ionization by the emitted electron. Of these effects, theoretical calculations indicate that the most damaging is caused by the change in nuclear charge as it affects the extranuclear electrons. An approximate theoretical retention value has been calculated and found to be in good agreement with the experimental result given above. This research represents a unique application of double labeling in the sense that one label is used as a tracer to detect the result of the decay of the other similar nucleus.

Work on liquid systems is exemplified by the following experiment. It was found that the organic phase retentions for the $(d, p),(n, 2 n)$, and $(\gamma, n)$ activation of iodine in liquid methyl and ethyl iodide, containing added iodine, were equal to the retention observed in radiative neutron capture (the $n, \gamma$ process). The results in a typical case are as follows: for ethyl iodide with 3.2 mole percent added iodine, the $\mathrm{I}^{127}(\mathrm{~d}, \mathrm{p}) \mathrm{I}^{12 k}$ reaction gave an organic retention of $29.5 \%$; the $\mathrm{I}^{12 \pi}(\mathrm{n}, 2 \mathrm{n}) \mathrm{I}^{126}$ reaction, $29.8 \%$; the $\mathrm{I}^{12 \pi}(\gamma, \mathrm{n}) \mathrm{I}^{126}$ reaction, $28.4 \%$. These values are to be contrasted with the $I^{127}(n, \gamma) I^{128}$ reaction retention of $28 \%$. This demonstrates that the chemical effects are essentially independent of the recoil energy of the activated nucleus, of the mode of nuclear formation, and of variation of fragmentation effects along the recoil track.

Several solid state systems have been investigated. In one, the Szilard-Chalmers reaction has been observed in a number of crystalline compounds of triand hexavalent chromium utilizing the $\mathrm{Cr}^{511}(\mathrm{n}, \gamma) \mathrm{Cr}^{51}$ reaction. In a series of alkali chromates and dichromates, the retention in the original oxidation state varied from 50 to 90 percent, whereas in ammonium chromate and dichromate the retention was much lower (18 to 32 percent). Presumably, the latter result is due to the possibility of the hot recoil frag. ment reacting with ammonium ion by an oxidationreduction process, which is not possible with the alkali salts.
Other solid systems under study involve the $\mathrm{N}^{14}(\mathrm{n}, \mathrm{p}) \mathrm{C}^{14}$ reaction in which the nitrogen is a constituent part of a given organic molecule. Thus the neutron irradiation of acridine has been shown to give acridine- $C^{14}$ and anthracene- $C^{14}$, the latter being removed by a carrier technique.

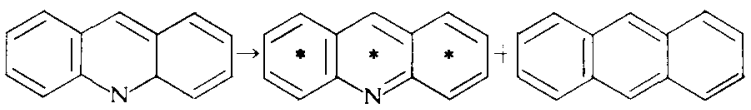

Of all the $\mathrm{C}^{14}$ produced in a given sample, about $3.5 \%$ is found in recovered acridine and $0.2 \%$ in the re-isolated anthracene which gives a ratio of about $18 / 1$, in rough agreement with the ratio of carbon to nitrogen atoms in the irradiated crystal.

In similar fashion, nicotinic acid on irradiation produces (among, of course, completely degraded as well as rearranged and polymeric materials) nicotinic acid- $C^{14}$ and its carbon analogue benzoic acid- $C^{14}$, the latter isolated by carrier (Figure 12). The ratio of activities here again is roughly equal to the carbonnitrogen ratio ( $7 / 1$ found, $6 / 1$ calculated). In addition, partial degradation of the benzoic acid places all the activity in the ring, with only a trace in the carboxyl carbon, which is strong but circumstantial evidence that the benzoic acid is labeled specifically in the meta position corresponding to the nitrogen position in the parent nicotinic acid. Should this hypothesis be confirmed, the potential usefulness of direct irradiation of nitrogen-containing organic substances for synthesis of specifically labeled compounds is evident.

\section{MOLECULAR STRUCTURE AND SOLID STATE}

The topics dealt with in this section refer mainly to problems in molecular structure and solid state. The experimental techniques, as well as the theoretical approaches, are, however, rather varied.

The earlier work on the low temperature absorption spectra of crystals of rare earth salts has continued. A study of the weak lines of $\mathrm{PrCl}_{3}$ as well as of $\operatorname{PrF}_{3}$ has resulted in a confirmation of the previous assignment of levels as well as the inversion of the normal energy level order in the ${ }^{3} \mathrm{~F}$ multiplet. Single crystals of europium chloride in lanthanum chloride were prepared, and the spectrum observed permitted an assignment of the $J$ values for absorption bands in the yellow, green and blue. This assignment indicates that the spectrum of a dilute aqueous solution of $\mathrm{EuCl}_{\text {: }}$ can be explained only by 

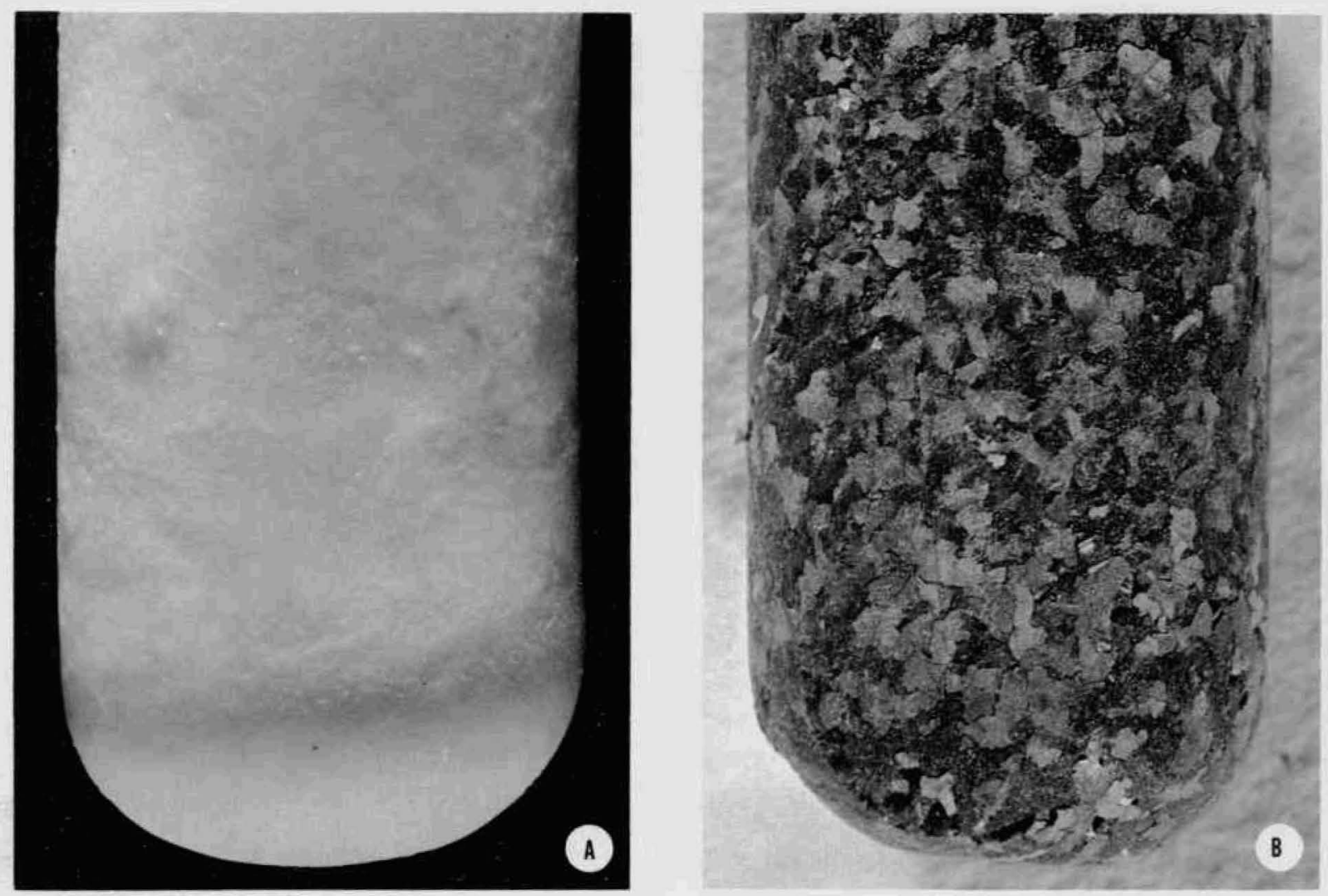

Figure 12. A sample of nicotinic acid: (A) before irradiation; (B) after irradiation in the nuclear reactor for three weeks at $\sim 10^{12} \mathrm{n} / \mathrm{sec} / \mathrm{cm}^{2}$ at $30^{\circ} \mathrm{C}$.

assuming $\mathrm{C}_{2 \mathrm{v}}$ symmetry about the $\mathrm{Eu}^{+3}$ ion in the solution.

The anhydrous chlorides of hexavalent (uranyl), quadrivalent, and trivalent uranium ions are photochemically active when in a solution fluid at $77^{\circ} \mathrm{K}$. It was shown both spectroscopically and chemically that quadrivalent uranium was reduced to the trivalent form, There were indications that tri- and hexavalent uranium were transformed photochemically to the tetravalent state.

Another application of these low temperature solvent techniques has been to chlorophyll b. A chlorophyll substance has been obtained along with chlorophyll $b^{\prime}$ when chlorophyll $b$ was heated in solvents and also without solvents, in the absence of oxygen and water. The spectrum, fluorescence and solvation properties are almost identical with chlorophyll $b$. Despite the fact that this substance does not undergo the Molisch phase test, reasons can be advanced that these three substances, $b, b^{\prime}$ and the new chlorophyll substance, may constitute the three possible tautomers of chlorophyll $b$ which can be im- agined by bonding the central magnesium atom in turn to three different pairs of pyrrol nitrogen atoms.

In connection with the chlorophyll studies, a theoretical investigation has been carried out on several prototype systems. Wheland's hetero-atom approximation to the LCAO-MO method has been used to calculate the ground state energies and spectra of the various isomers of porphyrin and dihydroporphyrin. The porphyrin calculations indicate that better agreement can be obtained with the observed spectrum by assuming a mixture of the two lower lying isomers (central hydrogens bonded to adjacent nitrogens, and central hydrogens bonded to opposite nitrogens) than can be obtained by assuming vibrational fine structure. The same approach was used in the dihydroporphyrin calculation, but in this case it appears that several of the observed bands are probably vibrational fine structures of a single electronic state.

Another theoretical study which has as its main object the evaluation of various approximations used in nonempirical molecular orbital theory has also 
been under way for some time. Dipole velocity and dipole length matrix elements have been investigated for several transitions in a number of conjugated organic systems, and it was shown that the introduction of configuration interaction greatly improves the validity of certain relations which are expected to exist between these matrix elements.

Computations have also been carried out with atomic orbitals having a Gaussian form rather than the usual hydrogenic Slater orbitals. In addition, a series of computations are now in progress on the $\mathrm{C}_{2}$ molecule in which many of the various approximations used in molecular orbital theory will be systematically applied to one system. Results obtained so far indicate that different approximations give vastly different answers.

An investigation has been undertaken to determine what happens to a molecular system when one of its constituent atoms undergoes nuclear decay (specifically $\beta^{ \pm}$decay, or $\mathrm{K}$ capture). The problem of electronic excitation due to the sudden change of charge of an atom is entirely similar to the same process in the atomic case and merely involves calculations of the overlapping of wave functions. The recoil, which in the atomic case leads to little or no excitation, does, however, in the molecular case lead to extensive excitation of rotations and vibrations which may lead to dissociation of the molecule. The recoil computations are actually applicable to any process in which a nucleus receives a recoil momentum (e.g., an $(\mathrm{n}, \gamma)$ process). An almost complete picture has now been obtained for electronic, vibrational, and rotational excitation for the case of $\mathrm{C}-\mathrm{C}^{14} \rightarrow \mathrm{C}-\mathrm{N}^{14+}+\beta^{-}$.

Calculations have been carried out of the inversion splitting and the rates of racemization in pyramidal $\mathrm{XY}_{3}$ molecules. The potential barrier for inversion is calculated by the method developed by Costain and Sutherland for ammonia. For all molecules, other than ammonia, containing $\mathrm{N}, \mathrm{P}, \mathrm{As}, \mathrm{Sb}$, and $S$ as the central atoms, it is concluded that the inversion splittings are too small to be measurable. From the calculated potential barriers, it is concluded that optically active compounds of arsenic, antimony, and sulfur should be stable to racemization at room temperature.

The program of magnetic susceptibility measurements has been devoted to an investigation of octahedral covalent complexes of the iron group elements. The magnetic moments of $\mathrm{K}_{3} \mathrm{Cr}(\mathrm{CN})_{6}$ and
$\mathrm{K}_{3} \mathrm{Mn}(\mathrm{CN})_{6}$ have been determined. These measurements show that the spin-orbit coupling is weaker in these substances than in the corresponding free ions, and that the magnetic moments may be calculated by the equation

$$
\mu=\sqrt{4 \mathrm{~S}(\mathrm{~S}+1)+\mathrm{L}(\mathrm{L}+1)}
$$

where $\mathrm{S}$ and $\mathrm{L}$ are chosen with the aid of Hund's rules and $M_{1}$ is restricted to the values, 1,0 , and -1 . The same calculation was shown to give excellent agreement for $\mathrm{Na}_{3} \mathrm{Fe}(\mathrm{CN})_{5} \mathrm{NO}_{2} \cdot 2 \mathrm{H}_{2} \mathrm{O}$.

Previously reported neutron diffraction measurements (BNL 246 (AS-7)) have confirmed the earlier $x$-ray result that zinc ferrite has the normal spinel structure, in which the diamagnetic zinc ions occupy the tetrahedral or $A$ sites and the trivalent iron ions occupy the octahedral or $B$ sites. As part of a continuing program of investigation of ferrites, studies have been made of the paramagnetic scattering and of the low temperature behavior of zinc ferrite. According to the Neel theory of ferrimagnetism, there is, in addition to the dominant antiferromagnetic interaction between magnetic ions on $A$ sites and those on $B$ sites a weaker antiferromagnetic coupling between magnetic moments on $B$ sites alone. Since the $A-B$ interaction is absent in zinc ferrite, this compound is ideally suited for the observation of the $B-B$ interaction and might well be expected to exhibit antiferromagnetism at sufficiently low temperatures.

Diffuse scattering of neutrons of wavelength $1.06 \mathrm{~A}$ has been studied with apparatus which permits measurement close to the forward direction under conditions of low and flat background. Corrections for multiple scattering were made by means of the calculations of Chandrasekhar and of Vineyard. (This procedure was tested by extensive measurements on beryllium, lead, bismuth, and thorium, in which multiple scattering is the only important diffuse scattering.) For zinc ferrite, the scattering at room temperature can be extrapolated to the theoretical value in the forward direction (i.e., unit form factor), and no reasonable extrapolation deviates from the theoretical value by more than about 3 percent. Figure 13 shows the variation of paramagnetic scattering cross section with angle. Excess scattering was observed at liquid nitrogen temperature and may perhaps be accounted for in terms of short range ferromagnetic order.

With the aid of a low temperature cryostat employing thin aluminum windows, Debye-Scherer dif- 


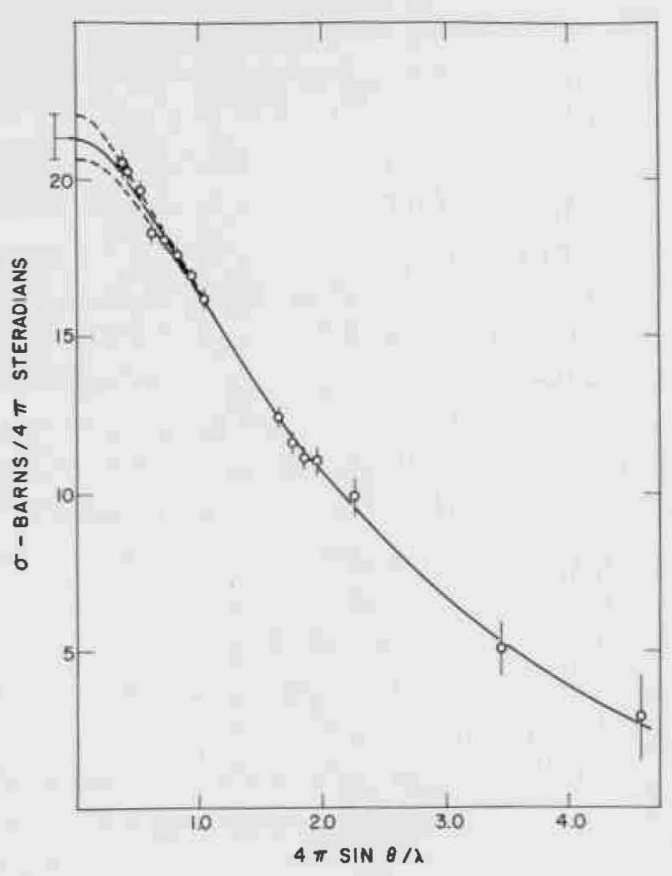

Figure 13. Magnetic scattering cross section per $\mathrm{Fe}^{3+}$ ion in $\mathrm{ZnFe}_{2} \mathrm{O}_{4}$.

fraction patterns of zinc ferrite were obtained from room temperature down to about $2.7^{\circ} \mathrm{K}$. A transition was observed to occur in the neighborhood of $9^{\circ} \mathrm{K}$. This was evidenced by the disappearance of the paramagnetic scattering and the simultaneous appearance of super lattice lines. Figure 14 shows a typical pattern taken at about $6^{\circ} \mathrm{K}$, in which approximately ten (mostly unresolved) lines can be seen. The absolute intensities and positions of the fundamental spinel lines are unchanged by the transition. The interpretation placed upon these observations is that a transition to an antiferromagnetic state has indeed occurred, with a Neel temperature of approximately $9^{\circ} \mathrm{K}$. The new lines can be indexed on a cubic cell whose edge is twice that of the original spinel unit cell. A tentative model for the antiferromagnetic structure has been obtained which results in agreement between calculated and observed intensities to about $\pm 20 \%$. A (001) projection of the proposed model showing only the 128 magnetic atoms is given in Figure 15. Each symbol in the figure represents a superposition of two spins, one from the upper and one from the lower half of the unit cell. The spin direction is along (010), and the sense of the individual spins is given by the appropriate sign in the diagram. The shaded symbols indicate that the sign changes in going from the lower to the upper half

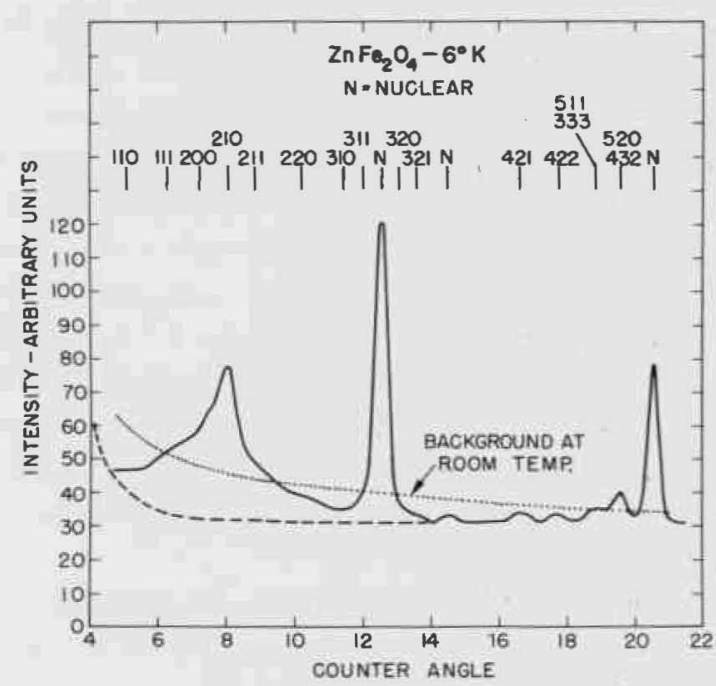

Figure 14.

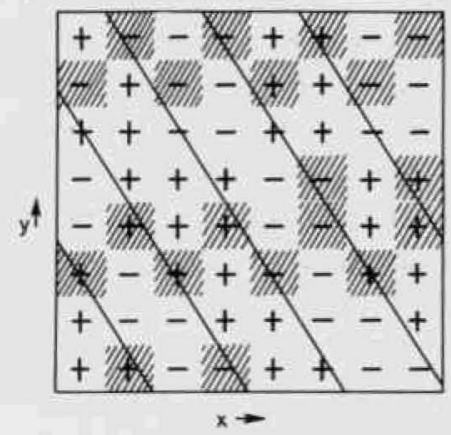

Figure 15. Schematic diagram of possible spin arrangement in antiferromagnetic zinc ferrite.

of the unit cell, and the unshaded symbols indicate that the spins are the same for the two halves. Qualitatively, the structure can be said to exhibit an antiferromagnetic alternation of ferromagnetic bands, each several atoms wide and nearly parallel to the (210) plane.

\section{ENGINEERING}

Research and development activities of an engineering character are carried out primarily in the Nuclear Engineering and Reactor Departments for problems associated with reactors and in the Accelerator Development Department for accelerators. The latter work has been described in an earlier section of this report. Also, although the work of the Mechanical Engineering Division is distributed through- 
out the entire Laboratory, its major portions are directed toward reactor and accelerator development. This section, therefore, is devoted primarily to a discussion of the research and development activities of the Nuclear Engineering and Reactor Departments. These two organizational units also provide technical services for the Laboratory. The Reactor Department operates the nuclear reactor, produces and distributes radioisotopes, and performs neutron service irradiations. The Nuclear Engineering Department operates a hot chemistry laboratory for highly radioactive chemical separations and provides gamma-irradiation services.

The Reactor Department does research in shielding and in certain neutron physics. Much of the latter work is of a cooperative nature and is carried out by guests from other institutions. The research in this area has been described in other appropriate sections of this report, e.g., work by guests from Columbia, as well as that by the Department's own staff members, is under Neutron Physics, and work by scientists from the General Electric Company and from the Atomic Energy Commission of Canada, Ltd. is under Solid State Physics. The cooperative work requiring the use of the reactor is under the general cognizance of the Reactor Department chairman.

The activities in the Nuclear Engineering Department have centered around continued research and development of various applications of the fission process for the eventual production of civilian power. About half the total effort of this Department has been directed toward the development of liquid metal fuel reactor (LMFR) systems. The other half was expended in studies of exponential assemblies of water and slightly enriched uranium, some special chemical processing systems undertaken in cooperation with other Atomic Energy Commission laboratories, development of special radioisotopes, problems involving the use and ultimate disposal of fission products from reactors, and two Ph.D. theses in heat transfer.

\section{LIQUID METAL FUEL REACTOR SYSTEMS}

Considerable progress in the development of the liquid metal fuel reactor (LMFR) components has been made during this year. Seven papers on the LMFR were declassified for presentation at the First International Congress on Nuclear Engineering, a meeting arranged by the Nuclear Engineering Divi- sion of the AIChE at Ann Arbor, Michigan in June. These papers were published as a group in the July, 1954 issue of Nucleonics. Since this much is now declassified, it seems worth while to give an over-all picture of these LMFR systems which, it is believed, offer some of the most promising means of achieving power from fission. Because the fuel is liquid, it can be moved by pumping instead of by the elaborate handling equipment needed for solid fuel elements. It is not subject to radiation damage, and it can be processed continuously for removal of fission products. The whole processing is included as an integral part of the reactor design. Because the fuel is a liquid metal, it can produce high temperature steam for the turbines and, hence, high thermodynamic efficiencies, without the problem of a high pressure container for the reactor core or blanket. It is also believed that such systems are sufficiently economic of neutrons that breeding of $\mathrm{U}^{233}$ from thorium is possible, which would thus result in a low fuel cost.

Figure 1 is a schematic diagram showing the core, heat exchanger, steam power generator, the two processing systems for the core, the blanket, and the blanket processing. The liquid metal fuel is a solution of $\mathrm{U}^{233}$ in bismuth. It was found that $\mathrm{U}$ is soluble to $5000 \mathrm{ppm}$ at $500^{\circ} \mathrm{C}$, whereas not more than 500 to $1000 \mathrm{ppm}$ are required of the reactor design. This dilution results in a high specific power. This liquid metal fuel is circulated through the core where heat of fission raises its temperature to about $520^{\circ} \mathrm{C}$; it then flows through heat exchangers producing superheated steam. The cooled fuel returns to the core to complete the cycle. The fission products will be removed in two ways. Any gases or vapors will be swept out (sparged) of the fuel stream into an evacuated space. The bismuth and polonium vapors will be caught on a cold trap and the iodine and xenon will go to a decay chamber where the gamma radiations can be used as a radiation source. A small fraction of the fuel will be circulated to the liquid fuel processing area where the rare earth fission products will be removed by contacting with fused salts. There has been considerable research and development on this process. The first fused salt tried was the low melting $\mathrm{LiCl}-\mathrm{KCl}$ eutectic. It now appears that the cross section and water content of $\mathrm{LiCl}$ make it undesirable. Some mixtures of the chlorides of $\mathrm{Na}$, $\mathrm{K}, \mathrm{Mg}, \mathrm{Ca}$, and $\mathrm{Zr}$ are more promising, and an attempt is being made to determine which is the best 

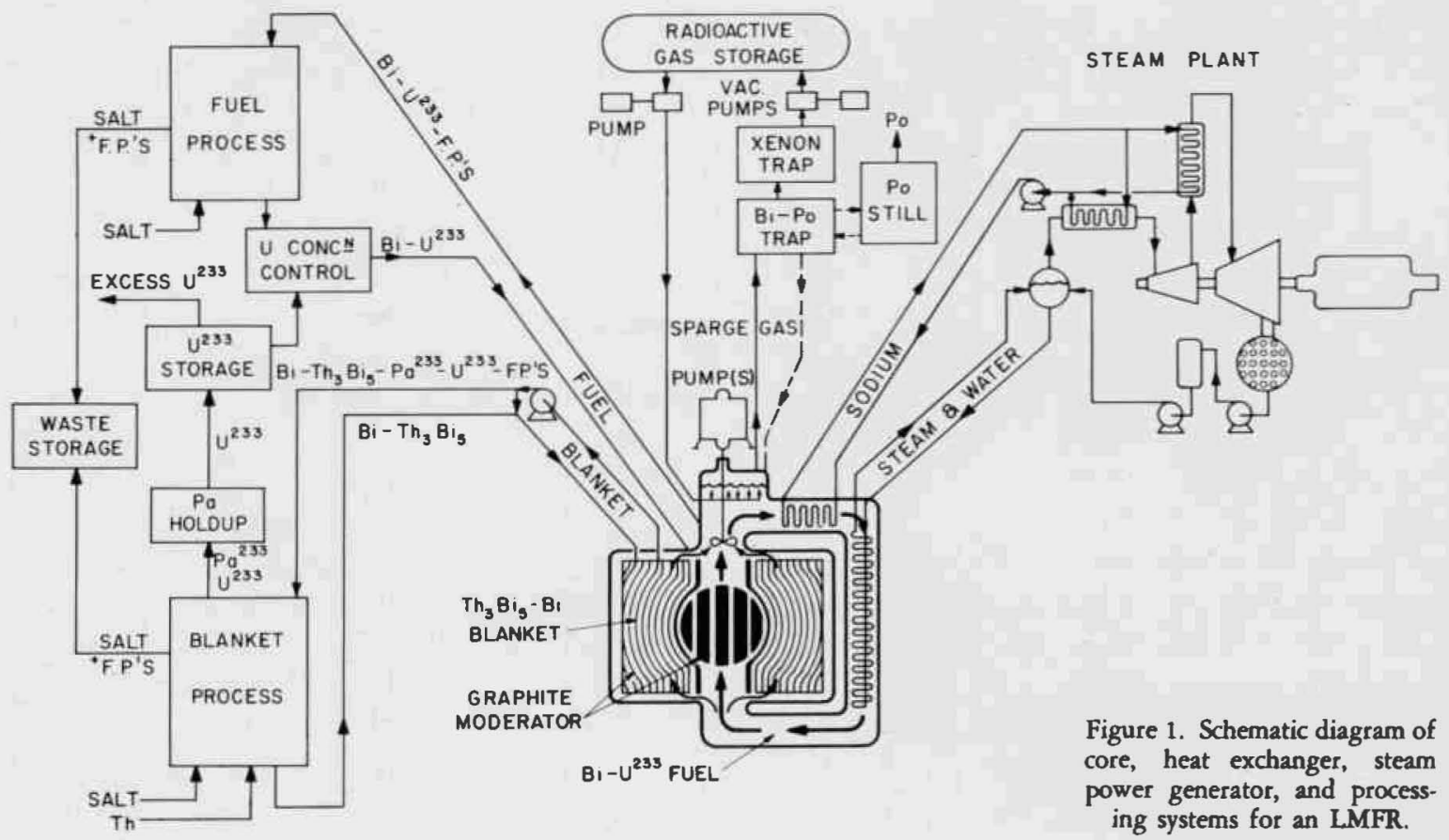

mixture. It would appear that by a proper choice the concentration of the fission products and the $\mathrm{U}^{233}$ can be adjusted to whatever value is desired.

Because liquid metal fuel is a rather unusual material, it was necessary to find a container material with a sufficiently low corrosion rate which would not be subject to mass transfer plugging that plagues many liquid metal systems. The Metallurgy Division has found that low chrome steels such as Croloy are entirely satisfactory if a few hundred $\mathrm{PPm}$ of $\mathrm{Mg}$ and $\mathrm{Zr}$ are added to the U-Bi solution as inhibitors. Such U-Bi solutions with the $\mathrm{Zr}$ and $\mathrm{Mg}$ additives have been circulated in a Croloy 5 -Si loop for some thousands of hours with no signs of plugging and essentially zero corrosion rate. It is proposed that graphite be used as the moderator and as the structural material for the blanket and core, the whole works to be enclosed in a low chrome steel tank. Although all indications are that the graphite and U-Bi will be compatible, studies are being continued to determine whether undesirable reactions take place at these temperatures under neutron and gamma radiations. A thorium blanket would surround the reactor core, absorb the excess neutrons, and convert the thorium into protactinium233 which decays to $U^{233}$. This $U^{233}$ can then be fed into the fuel stream to replace that consumed by fission. Extensive studies have been made on a tho- rium fluoride blanket which would be processed in place by gaseous fluorine and an in-pile loop has been constructed to test its operation. Although $\mathrm{U}^{233}$ and $\mathrm{Pa}$ could be extracted, problems of cooling such a blanket in a pile and of sintering the thorium fluoride which slows down the extraction rate of $\mathrm{Pa}$ and $\mathrm{U}^{233}$ have led to abandoning this attack for the LMFR. Work is now under way on a thorium bismuthide dispersion in liquid $\mathrm{Bi}$ as a blanket material to be circulated for cooling and processing.

Considerable engineering development work is being done in circulating $\mathrm{Bi}$ and $\mathrm{U}-\mathrm{Bi}$ solutions in loops so that an in-pile processing loop can be set up. Some of these loops (Figure 2) have circulated $\mathrm{U}$-Bi solutions for as long as $14,000 \mathrm{hr}$ and are still running. The loop which will circulate the U-Bi solution in an experimental hole in the Brookhaven reactor and process the solution for $\mathrm{Xe}$ and rare earth fission products is built and nearly ready for in-pile operation.

Various dispersions of $\mathrm{U}, \mathrm{Bi}, \mathrm{Sn}, \mathrm{Pb}$ alloys in liquid $\mathrm{Bi}, \mathrm{Pb}, \mathrm{Sn}$ alloys have also been studied. These dispersions can carry higher concentrations of $\mathrm{U}$ than the solution and might have applications in other reactor designs. However, at the moment most of the work on the phase diagrams of such mixtures is confined to the thorium bismuthide in bismuth dispersion for use as a blanket. 


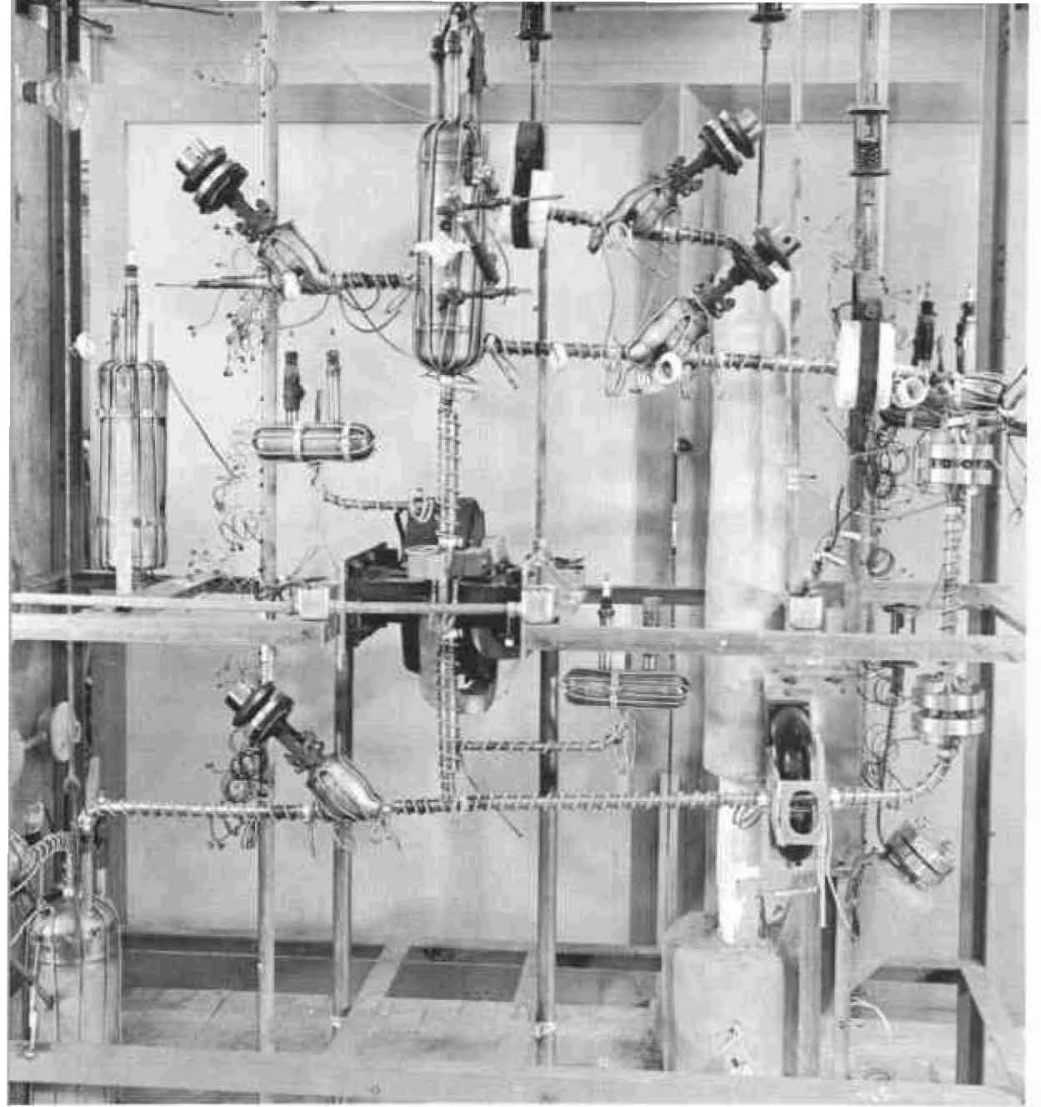

Figure 2. Flow diagram and photograph of an out-ofpile test loop. In the photograph, the loop is shown before insulation was applied.

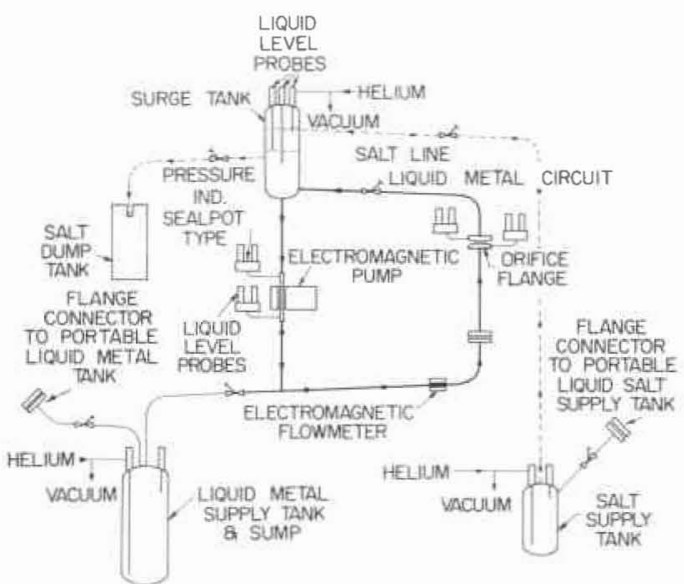

It should be emphasized that the above description is not a design for a specific reactor but merely a scheme for developing a reactor design from the component studies that have been going on at Brookhaven Laboratory.

In addition to the unclassified references above, the material on LMFR components as well as the work on reactor physics and other chemical processing developments is covered in the quarterly classified progress reports.

\section{REACTOR PHYSICS}

Experiments have been continued on exponential lattices of slightly enriched uranium in water. This work is aimed at measurement of parameters needed for predicting the neutron economy of these fuelmoderator systems. New experimental methods have been developed for some of the parameters, and the theory of these lattices has been extended and refined. The properties of these lattices are better understood than before with the result that the design of reactors based on slightly enriched uranium - water lattices is now on a sounder basis. This work is being continued.

The theoretical investigation of liquid metal fuel reactors has been continued and extended. In addition to refined calculations of the neutron and fis- sionable material economies, studies of the hydrodynamic stability and control problems are under way.

Studies have been started on the problems associated with the proposed replacement of the fuel elements in the Brookhaven pile by enriched uranium elements.

\section{ULTIMATE WASTE DISPOSAL}

During the past year a pilot plant was constructed to determine the general workability and some of the essential economic factors in the process for adsorbing and fixing fission products on montmorillonite clay. The process is designed to accomplish a basic step in the ultimate disposal of fission product wastes or the preparation of intense radiation sources. The pilot plant is virtually complete except that the semipermeable membrane unit has not been installed.

The results of numerous experiments on the use of semi-permeable membranes for neutralization of nitric acid and, similarly, for treatment of other nitrate salts in reactor fuel processing waste streams lend considerable support to the value of these membranes to be used in conjunction with the clay columns. Radiation effects on the membranes do not appear to be serious within practical limits.

The practicability of removing aluminum from nitrate solutions by electro deposition without the 
addition of other cations has been demonstrated. A considerably greater reduction in the quantity of solids destined for disposal than that of other methods of precipitation is thus obtained. The aluminum is then converted to the stable oxide by heating to high temperatures. Two stable end products for containing the fission products are thus available for use as radiation sources or for final disposal, namely, aluminum oxide and montmorillonite clay (heattreated).

\section{SHIELDING}

Shielding studies carried out by the Reactor Department are directed at measurement and analysis of the transmission of neutrons and gamma rays through various materials and configurations. This program provides both theoretical and experimental guidance for specific endeavors in which shielding information is required. One of its immediate goals is the minimizing of time-consuming and costly mock-up tests. Experiments are undertaken which should result in a better understanding of the processes by which radiations are attenuated and should provide computational methods, technical data, and experimental verifications which can be used with confidence by reactor design engineers. A portion of this work is reported in the quarterly classified report of the Laboratory. In addition to the work under the formal program, the shielding group has handled a number of inquiries from industrial organizations; some have requested specific information and advice with respect to shielding problems, and others have requested testing and evaluation of materials having a possible application in shielding. Several specific experiments are reported in the following paragraphs.

Gamma rays in the energy range of 0.1 to $2 \mathrm{Mev}$ which resulted from thermal neutron capture in several elements were studied with a scintillation spectrometer. Use was made both of single crystal data and of supplemental results obtained by the Compton scattering techniques of Hofstadter and McIntyre. Elements studied included $\mathrm{V}, \mathrm{Co}, \mathrm{Cu}, \mathrm{Ti}, \mathrm{Fe}, \mathrm{Cr}$, $\mathrm{Au}$, and I. In some cases, evidence was found for weak lines, previously unreported, which have significance for proposed decay schemes.

The gamma-ray dose resulting from the penetration of $\mathrm{Na}^{24}$ gamma rays through steel, lucite, and lead was studied as a function of the angle of incidence of the gamma rays on slabs of varying thick- ness. The results for lucite and steel are reported in the minutes of the November 1953 Shielding Information Meeting. The data for lead, not previously reported because of certain experimental inconsistencies, have been corrected and will be presented in a forthcoming report which will also include the steel and lucite results.

\section{HEAT TRANSFER STUDIES}

\section{Cross-Flow Heat Transfer with Mereury}

During the past fiscal year, experimental heat transfer results were obtained for cross flow of mercury through a staggered tube bank. These results are applicable to the design of heat exchanger equipment used for both the alkali and heavy metals. For individual tubes located in different parts of the bank, the circumferential variation of the heat transfer coefficient was measured. From these local coefficients, average coefficients for the individual tubes and for the tube bank as a whole were obtained. The chief variables studied were velocity, tube location, angle around tube, and degree of wetting. For wetting and non-wetting conditions, the tube surfaces used were copper and chromium plated copper, respectively.

The scope and conditions of the investigation are summarized in Table 1 and the results may be stated as follows:

1.) Under non-wetting conditions, the average coefficient for the interior of the tube bank varied as the 0.52 power of the Reynolds number, whereas for wetting conditions it varied as the 0.66 power.

2.) At a Reynolds number of 50,000 , the average coefficient for wetting conditions was about 4300 $\mathrm{Btu} /(\mathrm{hr})(\mathrm{ft})^{2}\left({ }^{\circ} \mathrm{F}\right)$, whereas it was only about 3200 for non-wetting conditions.

3.) For a given tube, the local coefficient was at a maximum at the forward stagnation point and gradually decreased to a minimum at the rear stagnation point. The coefficient at the front was roughly twice as great as that at the rear.

4.) Observed pressure drops across the tube bank, under non-wetting conditions, were appreciably less than expected on the basis of results on oil and water reported in the literature.

It is planned to continue the experimental work at considerably higher values of the Reynolds num- 
Table 1

\begin{tabular}{ll}
\hline Tube size, OD & $0.500 \mathrm{in}$. \\
Length of tubes, vertical & $7.0 \mathrm{in}$. \\
Tube spacing & Equilateral triangle \\
Tube pitch & $11 / 16 \mathrm{in}$. \\
(Pitch) $/$ (Diameter) & 1.375 \\
Tube bank size & 7 tubes wide $\times 10$ tubes deep \\
$\begin{array}{l}\text { Bulk mercury temperature } \\
\text { Maximum temperature rise }\end{array}$ & $100^{\circ} \mathrm{F}$ (approx.) \\
$\begin{array}{l}\text { of mercury in passing } \\
\text { through tube bank }\end{array}$ & $1 /{ }^{\circ} \mathrm{F}$ (approx.) \\
$\begin{array}{l}\text { Prandtl number } \\
\text { Reynolds number range } \\
\text { Linear velocity range, } \mathrm{V}\end{array}$ & 0.023 15,000 to 83,000 \\
\hline
\end{tabular}

ber and also to increase the amount of data available under wetting conditions.

\section{Heat Transfer in Spray Columns}

During the past year, a Ph.D. thesis project has been started to study heat transfer from mercury to water in a spray column. In this investigation, mercury is sprayed into a rising column of water, and heat transfer coefficients are presently being measured under different operating conditions. The two major variables are droplet size and mercury holdup. The droplet size, linear velocity of droplets, and kinetics of flow are determined by means of high speed photography.

\section{FISSION PRODUCTS UTILIZATION PROJECT}

\section{Radiation Application Research}

Polymerization Studies - The solid state polymerization of acrylamide has been continued, and the study is being extended to other solid monomers. The rate of polymerization of acrylamide was determined at three gamma-ray intensities between 93,000 $\mathrm{r} / \mathrm{hr}$ and $435,000 \mathrm{r} / \mathrm{hr}$. The rate is dependent on intensity to the 0.75 power. The monomer dependence has been established to be between zero and onehalf power. Equations have been developed that are compatible with one-half and first-order intensity dependence and a monomer dependence between zero and one-half.

$$
\begin{aligned}
& \frac{-\mathrm{dM}}{\mathrm{dt}}=\mathrm{k}_{\mathrm{p}} \mathrm{k}_{\mathrm{i}}(\mathrm{I})\left(\frac{\mathrm{k}_{\mathrm{g}}-\mathrm{k}_{3}}{\mathrm{k}_{\mathrm{t}}} \mathrm{M}+\frac{\mathrm{k}_{3}}{\mathrm{k}_{\mathrm{t}}}\right)^{1 / 2} \\
& \frac{-\mathrm{dM}}{\mathrm{dt}}=1_{\mathrm{p}} 1^{1 / 2}\left(\frac{\mathrm{k}_{2}-\mathrm{k}_{3}}{\mathrm{k}_{\mathrm{t}}} \mathrm{M}+\frac{\mathrm{k}_{3}}{\mathrm{k}_{\mathrm{t}}}\right)^{1 / 2}
\end{aligned}
$$

Equation (1) assumes that a) polymerization occurs in discrete tracks, the number of which is proportional to intensity $\left(k_{1} \mathrm{I}\right)$; b) termination occurs bimolecularly in tracks; c) initiation of radicals can occur by gamma interaction with monomer $\left(\mathrm{k}_{2} \mathrm{M}\right)$ or polymer $\left(\mathrm{k}_{3} \mathrm{P}\right)$; and $\mathrm{d}$ ) the propagation step is independent of monomer concentration. Equation (2) assumes that a) polymerization occurs uniformly; b) by interaction of gamma with monomer or polymer; c) termination is bimolecular; and d) the propagation step is independent of monomer concentration.

Molecular weight increases with conversion which indicates that branching is appreciable. From viscosity measurements and osmotic pressure determination the equation $[\eta]=8.27 \times 10^{-2} \mathrm{Mn}^{0.28}$ was developed. The low value of the exponent 0.28 confirms the occurrence of appreciable branching.

The polymerization rate of methacrylamide at $35^{\circ} \mathrm{C}$ and $250,000 \mathrm{r} / \mathrm{hr}$ intensity was determined to be 0.7 percent per hour and for methylene-bisacrylamide, under similar conditions it was 1.6 percent per hour.

The n-vinylpyrollidone polymerization rate has been found to be independent of monomer concentration between 1 percent and 20 percent concentration in water. It was found that the rate decreases and the viscosity of the polymer decreases as the water solvent is replaced by increasing amounts of methanol. Appropriate viscosities can best be obtained by change in monomer concentration and solvent composition variables. Samples of the polymer have been fractionated and compared with commercial polyvinylpyrollidone and a narrower molecular

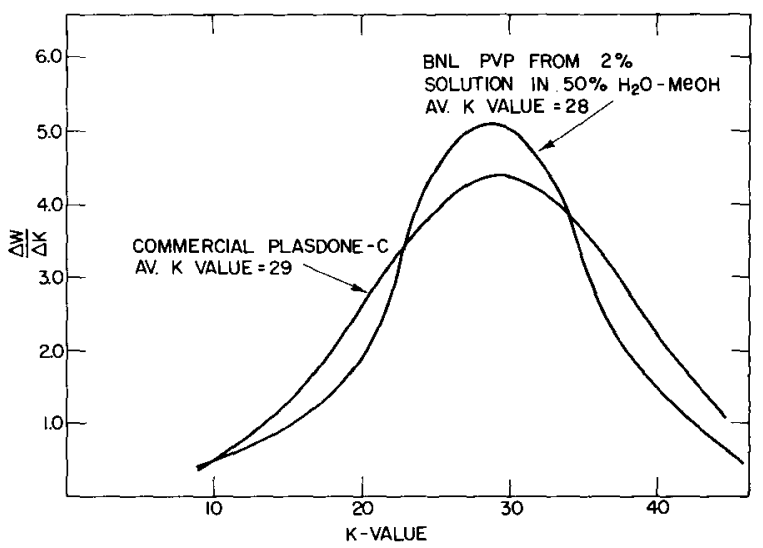

Figure 3. Differential weight distribution curve for polyvinylpyrollidone. 
weight distribution has been obtained (Figure 3). Narrow molecular weight distribution is an important improvement on the commercial plasdone in that it may permit the blood plasma substitute to be completely eliminated by the kidney.

Gamma irradiation of commercial polyethylene indicated that heat distortion properties are improved with increasing dosage and that tensile and elongation properties are adversely affected. Infrared studies indicated the presence of hydroxyl and carbonyl groups, which suggests that surface oxidation also is occurring.

Kinetic studies of extremely pure polystyrene indicate that at low intensities $(<100,000 \mathrm{r} / \mathrm{hr})$ the polymerization rate is dependent on the intensity to the one-half power and at high intensities $(>100,000 \mathrm{r} / \mathrm{hr}$ ) the polymerization rate is linearly dependent on intensity.

\section{SPECIAL RADIOISOTOPES DEVELOPMENT}

\section{lodine-132}

During the year $I^{132}(2.33$-hr half-life) passed from the development phase through a period of clinical testing to the production phase. In October 1953, a series of shipments of this isotope to the Sloan-Kettering Institute for Cancer Research was started and has continued to date. In cooperation with the Laboratory, a group at S-KI under the direction of Dr. J. E. Rall has been evaluating the use of this isotope for clinical diagnosis. The results of these investigations indicate that this isotope has definite merit, and as a consequence of this study it was decided to offer $\mathrm{I}^{132}$ to qualified users at large.

A total of 11 shipments, averaging over 100 millicuries each, has been made to the Sloan-Kettering group. Some $\mathrm{I}^{132}$ was also supplied to the Brookhaven Medical Department. The shipping unit has continued to function nicely, requiring only one minor repair after being in operation almost continuously for a period of six months.

\section{lodine-133}

At the request of another group at the SloanKettering Institute, a simple convenient process for preparing fission product $\mathrm{I}^{133}$ (21-hr half-life) has been devised. The scheme uses direct-controlled oxidation of an irradiated uranium foil. The product iodine is separated from the uranium and other fission products about $21 \mathrm{hr}$ after removal from the reactor. After another $27 \mathrm{hr}$ all the short-lived iodine isotopes have died out which leaves only $\mathrm{I}^{133}$ and $\mathrm{I}^{131}$, at which time the ratio of these two activities is about $6: 1$.

Seventeen batches have already been shipped to this Sloan-Kettering group, the recent ones each containing over $50 \mathrm{mc}$ upon arrival in New York City. $A$ request from a second group has been received for a continuing supply of $100 \mathrm{mc}$ batches. The process is being scaled up to meet this demand, and the still larger demand that is anticipated when this isotope is offered to qualified users at large. Actual yield at the present time is about 60 percent of theoretical, and development work is continuing to improve this yield and make it more reproducible.

\section{Ytrium-90}

Work was undertaken to develop a simple milking system whereby this very important $\beta^{-}$-emitting isotope (65-hr half-life) could be separated from its long-lived $\beta^{-}$-emitting strontium parent. Although this work has not been completed, nothing has appeared to indicate that it will not be concluded successfully at an early date.

In response to requests from members of the medical profession for clay beads containing $\mathrm{Y}^{90}$, the mechanics of a process have been worked out for making such beads of 0.033 to 0.036 -in. diameter, each containing essentially pure $\mathrm{Y}^{90}$ activity of the order of $0.5 \mathrm{mc}$. Work has also been started on a process for making such beads of $s_{\mu}$ diameter.

\section{Fluorine-18}

The work of Knight et al (Oak Ridge) was repeated and confirmed; tritons obtained from neutron irradiation of $\mathrm{LiNO}_{3}$ were used to give a $(t, n)$ reaction on $\mathrm{O}^{16}$, and, as calculated, it was possible to produce millicurie quantities of $\mathrm{F}^{18}$ from gram quantities of $\mathrm{LiNO}_{3}$. A process was devised to give a carrier-free solution of $\mathrm{F}^{18}$.

\section{Gallium-72}

In an effort to make millicurie quantities of carrier-free $\mathrm{Ga}^{72}$, tracer experiments were performed on the $\mathrm{Ge}^{72}(\mathrm{n}, \mathrm{p}) \mathrm{Ga}^{72}$ reaction. However, the yield of $\mathrm{Ga}^{72}$ was of the order of $0.5 \mu \mathrm{c}$ per gram of $\mathrm{GeO}_{2}$ at saturation in a position in the Brookhaven reactor (pneumatic tube) where the thermal neutron flux is of the order of $3 \times 10^{12}$. This yield is too small to be of practical importance and the work was dropped. 


\section{Arsenic-77}

As a by-product of the work on $\mathrm{Ga}^{72}$, information has been obtained on which to base a simple rapid process for preparation of carrier-free $39-\mathrm{hr} A^{i \pi}$ in relatively large amounts. This process, however, will not be developed further unless there is a greater demand for this isotope than there seems to be at the present. 


\section{LIFE SCIENCES}

\section{BIOLOGY}

The program of the Department has continued along the lines already established. During the year no new major facilities have been added to the Department, but the existing facilities have been used to capacity.

The research activities of the Department continue to center around the special facilities of the Laboratory. In general this involves (1) the use of radiations of various kinds in the study of their effects on biological material and also as a tool for the study of life processes; and (2) the use of radioactive tracers in studies of life processes.

In the first category the gamma field continues to be extremely valuable. The abnormalities of the plants grown in the field are still under study and probably will be for a number of years. The thermal neutron facility has been used extensively; during the year it was necessary to arrange to have it in use 24 hours a day 7 days a week, in order to keep up with the demand.

The summer program has continued to grow and is now an important part of the research and educational activities of the Department. This summer (1954) there are 17 scientists and 25 students working in the Department. Thus the scientific activities of the Department are nearly doubled during the summer.

The scientific work of the Department can conveniently be discussed under six headings as follows:

\section{PLANT PHYSIOLOGY}

The number of interesting radiation reactions have already been reported in plants and more continue to be found and studied. Each plant species appears to have a characteristic reaction to radiation. Some produce large quantities of adventitious roots, in others the characteristic growth patterns are drastically altered, others seem to be stimulated at certain dosage rates, etc. The attempt is being made to find some basis for the different reactions and to classify plants in such a way that a. reasonable hypothesis for radiation reactions can be formulated.
One of the most interesting observations made in this program recently is that certain tobacco plants which normally exhibit only a few spontaneous tumors develop enormous numbers of tumors when exposed to radiation. In some cases almost the entire plant appears to be tumor tissue (Figure 1). It has been known for many years that radiations produce tumors in animals; this is the first clear case of radiation-induced carcinogenesis in plants.

This field of research is just opening up, and the ground work is now being done. The effect is being described in detail in a number of plants - a necessary first step in exploiting the phenomenon. It is hoped that soon the physiological aspects of the phenomenon can be explored, and this technique can be used in fundamental studies of growth and differentiation.

Supplementing this work, there is a good deal of work in progress on attempts to find the effect of different radiations on cells and cellular growth. Essentially this is a comparison of the difference in biological effect between thermal neutrons and $\mathbf{x}$-rays. Surprisingly enough, there are qualitative as well as quantitative differences between the different radiations. Last year it was reported that there were differences in the uniformity of the growth pattern depending on the type of radiation used. In analyzing this effect, it was found that there are differences in growth response following different kinds of irradiation depending on the gaseous atmosphere of the seeds and upon the water content of the seeds. It now appears that even pretreating seeds with water and drying the seeds back to normal produces profound sensitivity differences for $\mathrm{x}$-rays but not for thermal neutrons.

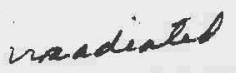

Figure 1. Tumors on a tobacco plant. The plant on the right was photographed 147 days after being placed in the gamma greenhouse as a seedling receiving radiation at the rate of $200 \mathrm{r}$ per day. The tumors are so numerous that almost all of the plant is tumor tissue. The control plant is on the left.

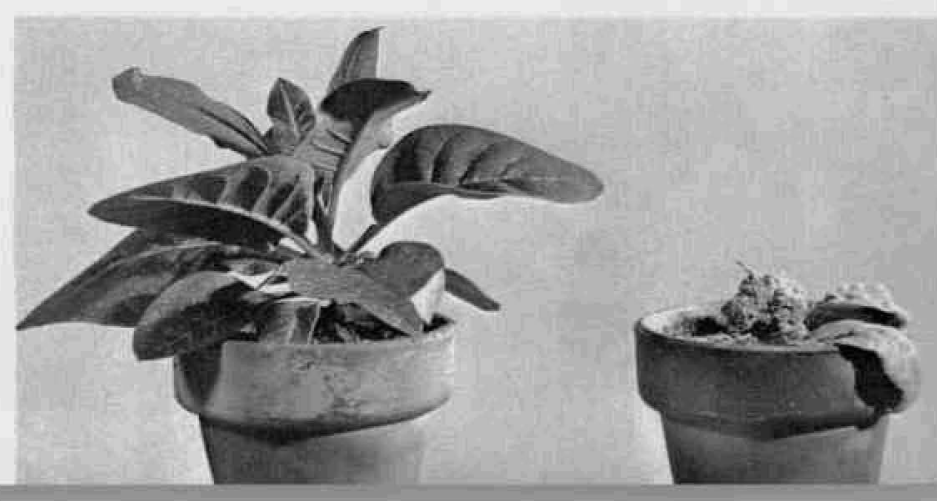



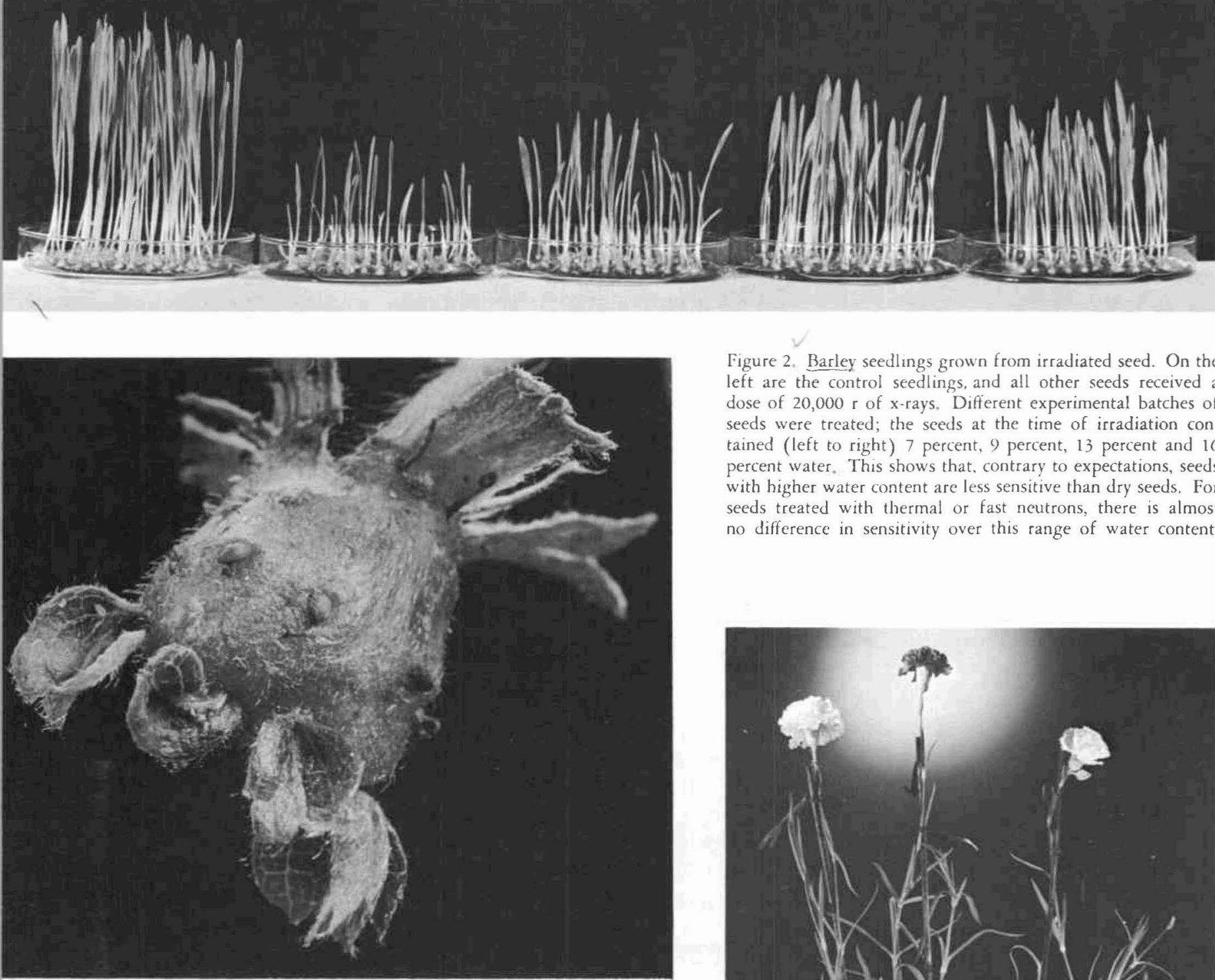

Figure 2, Barley seedlings grown from irradiated seed. On the left are the control seedlings, and all other seeds received a dose of 20,000 r of x-rays. Different experimental batches of seeds were treated; the sceds at the time of irradiation contained (left to right) 7 percent, 9 percent, 13 percent and 16 percent water. This shows that, contrary to expectations, seeds with higher water content are less sensitive than dry seeds. For seeds treated with thermal or fast neutrons, there is almost no difference in sensitivity over this range of water content.

Figure 4. Strawberry grown in the gamma field shows leaves growing from the fruit - a very common occurence in straw. berries grown at a particular distance from the source. This is part of the somatic mutation program; these plants belong to Dr. Russell Eggert of the University of New Hampshire.

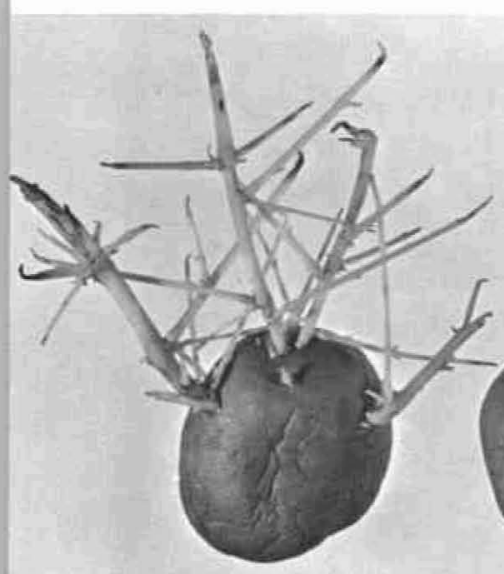

CONTROL

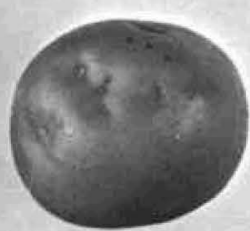

$20,000 \mathrm{r}$
Figure 5. Potato tubers photographed 15 months after $\mathrm{x}$-ray treatment at the doses indicated. The tubers were stored under standard conditions, and it will be seen that $\mathrm{x}$-irradiation has greatly improved the keeping qualities of the potatoes.

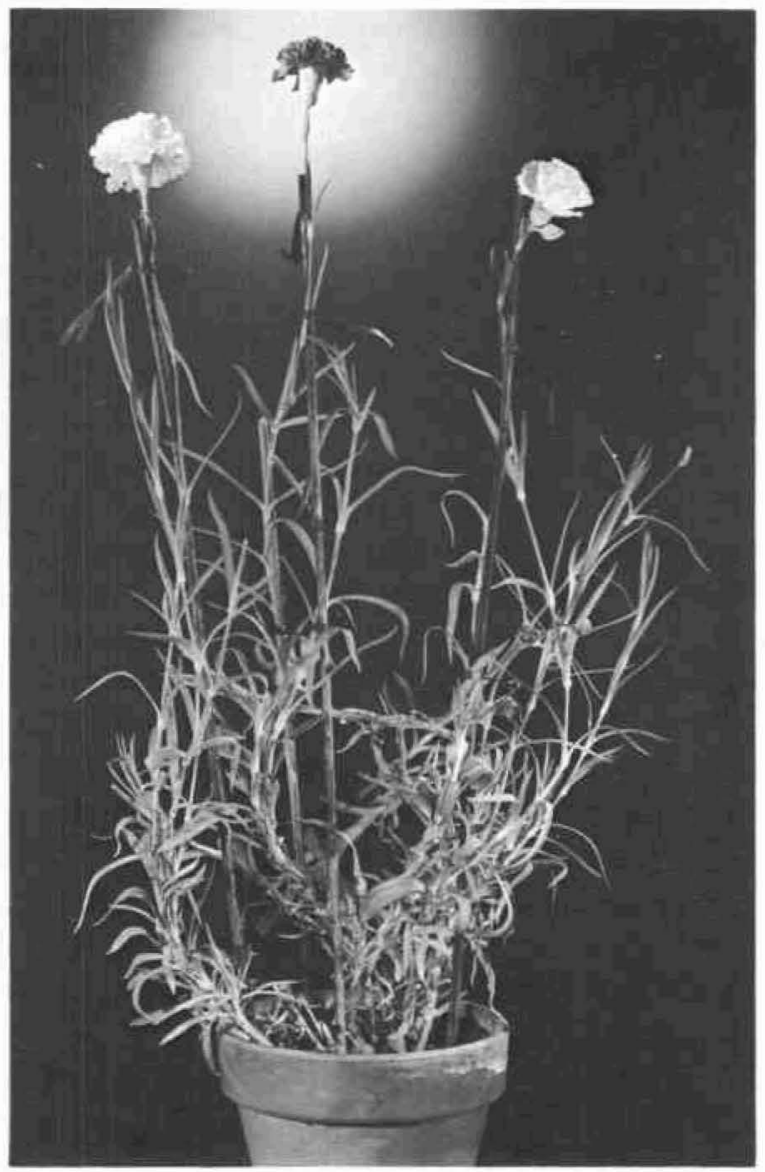

Figure 3. Carnation plant which has been grown in the gamma field. A somatic mutation has occured in this plant to give a red flower on a white flowering plant. Cuttings grown from the stalk having the red flower gave a plant which produced all red flowers. In this way, the red flower can be propogated indefinitely but it is doubtful if the seed from this flower would breed true. 
Recently a careful study of the sensitivity of seeds to radiation has been undertaken as a function of water content (Figure 2). For fast neutrons the sensitivity increases with increasing water content, probably because of the higher hydrogen concentration available for recoil reactions. For thermal neutrons there is a lowered sensitivity as the water content of the seeds is increased. This is probably due to the lower concentration of nitrogen and boron available for neutron capture reactions. When $\mathrm{x}$-rays are used, it is found that there is at first a drop in sensitivity as the water content of the seed is increased up to about 16 percent, but at higher concentrations the sensitivity is greater. It has been commonly accepted that increased water content raises the sensitivity of biological systems to $x$-rays because it favors greater active radical production which initiates oxidation and reduction at sensitive sites. These data offer a serious complication to the unequivocal acceptance of this postulate.

Changes in cellular damage are being followed for all these radiations and it is interesting to note that chromosome damage is by no means parallel to the observed inhibition of growth. In general it can be said that neutrons cause relatively more damage to the nucleus for a given growth inhibition than $\mathrm{x}$-rays.

As a corollary to this work, experiments are in progress to determine the chemical factors involved in chromosome abnormalities. A low magnesium or calcium ion concentration in the nutrient supplied to the plant will cause as much as a 20 -fold increase in chromosome abnormalities, both for normal and for irradiated plants.

As part of the research in plant physiology, a plant breeding program is being conducted in conjunction with 17 universities and agricultural experiment stations. Almost half the gamma field is now being utilized for this project, and nearly half the time of the thermal column. The object is to attempt to produce somatic mutations in plants and trees which are normally propagated asexually and, by screening the mutations, to select the economically important ones. It is too early yet to expect significant results, but already a number of mutations have been observed. The most striking of these is a red flowering carnation from a pure white plant (Figure 3). Many weird growth abnormalities have been observed on other plants and trees (Figure 4), which would lead to the belief that many mutations will appear in a few years.
It was observed several years ago in connection with some other work that potato tubers which had been subjected to $x$-irradiation did not spoil as quickly as normal tubers. An experiment was designed to verify this observation and to express the effect in quantitative terms. A batch of tubers was divided into six parts and each part was given a dose of $\mathrm{x}$-rays of between zero and 106,000 r. They were then stored under standard storage conditions and periodically examined and samples subjected to taste tests. Even after 15 months in storage (Figure 5) tubers which had received 20,000 r were excellent potatoes in every respect. This result is obviously of considerable practical importance, and the experiments are being turned over to the Long Island Vegetable Research Farm at Riverhead to continue.

The photosynthetic reaction is certainly the most important of all chemical reactions, and probably one of the least understood. Since the forward reaction of the conversion of carbon dioxide and water glucose proceeds so fast that it is difficult to follow, during the past three years work in this laboratory has concentrated on the reverse reactions, i.e., the glucose metabolism of the plant. It has been found that there are two alternate pathways for the degradation of glucose. Many of the intermediates and reaction mechanisms have been worked out, and these presumably apply to the forward reaction also. One extremely interesting result has been the finding that there is a different mechanism for metabolism in young and old tissue. In young, rapidly growing tissue, respiration is predominantly by the EmbdenMeyerhof-Parnas scheme, but as the tissue grows older there is a shift to the "oxidative pathway." This has now been found for several plants and seems to be true for both stems and roots.

\section{GENETICS}

Corn has been extensively used in this laboratory in genetic studies because its genetic structure is so well known and because it is such a convenient material to work with from the radiation point of view. By the use of the gamma field it has been possible to determine the mutation rate as a function of the radiation dosage rate for four genetic loci in corn. The results have given positive proof that the mutation rate is not a linear function of the dosage rate as has always been supposed but increases rather sharply with increasing dosage rate. One must either conclude that the mutation process is not a 


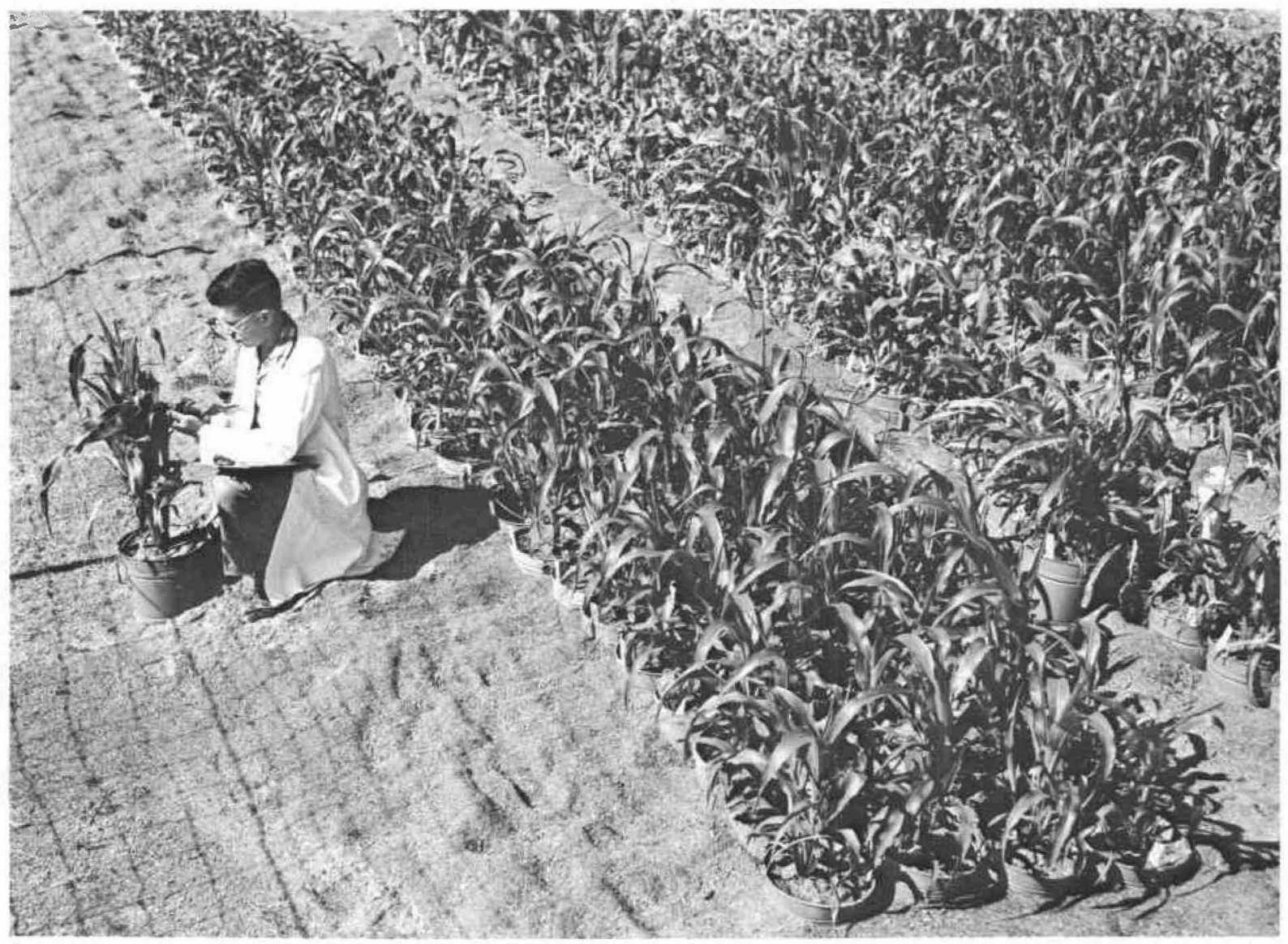

Figure 6. Portable corn field. The corn plants in this field are grown in buckets and transferred to the gamma field for definite periods of time. This technique is used to determine the time, during pollen development, at which the cell is most susceptible to mutation.

"one hit" process, as postulated by the hit theory, or that the hit theory does not apply and some other mechanism must be responsible for radiation-induced genetic change.

A technique of irradiating corn plants for relatively short periods of time has been perfected which makes use of a "portable corn field" (Figure 6) by means of which com plants are put in and taken out of the gamma field. This technique enables one to irradiate the plants during predetermined periods in the maturation cycle of the germinal cells. The results indicate that there is an enormous increase in sensitivity-during a particular stage of maturation and thereafter the sensitivity drops markedly (Figure 7). Present indications are that cells which have been subjected to radiation during the critical period and also during subsequent periods will show a decreased mutation rate over those that were irradiated during sensitive periods and then removed from the radiation field. The indication would seem to be that the radiations produce a physiological as well as a genetic effect on the germinal cells and that the maximum genetic effect can be expressed only when the physiological conditions are optimal.

This work has also involved the perfection of techniques for irradiating corn pollen with thermal neutrons. Present indications are that the thermal neutrons are between two and four times more effective as a mutagenic agent than $\mathrm{x}$-rays and that for the rapid production of mutations this technique may prove to be considerably more important than the gamma field technique.

Work is continuing on the production of a dwarf variety of corn. A number of radiation-induced dwarfed varieties have now been produced and work is in progress both by conventional plant breeding methods and radiation-induced mutations to increase the yield of this dwarfed corn so that it will be comparable to the yield on tall corn but will require a good deal less fertilizer. 


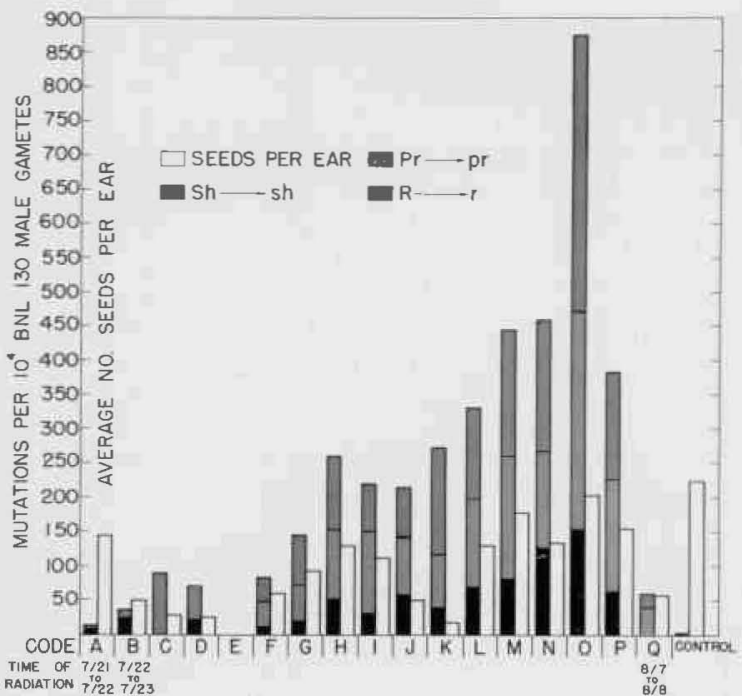

Figure 7. Chart showing mutations produced in corn pollen by irradiating the plant in the gamma field for one day at different times during pollen development. Each code letter represents one day in pollen development. Tassels irradiated shortly before shedding $(\mathrm{O})$ produce most mutations, while irradiation prior to meiosis (E) produces very few.

In addition to the work on corn an important investigation has been undertaken on the mutagenic effects of radiation on seeds. Barley and oats were used principally in this study. It has been found that the irradiation of seeds will produce many mutations in the offspring and the mutation rate can be increased several hundredfold this way over the spontaneous mutation rate. In general, the kinds of mutations which one observes are the same as those observed in spontaneous mutations and this is true for $\mathrm{x}$-rays, gamma rays and thermal neutrons. However, quantitative differences have been observed between the irradiated seeds and the controls and between the effects of the different radiations.

Perhaps the most important feature of this work is the finding that at least some of the mutations produced in this way are dominant mutations. Up to the present time it had always been assumed that radiation would destroy genes and therefore one could not expect to get anything except a recessive mutation by irradiation. Since this has now been shown definitely to be not true, it will be necessary to alter our thinking completely about the nature of radiation-induced mutations and indeed about the entire mutation process.

In the course of similar work, a careful comparison was made between the number of mutations it is possible to get from seeds irradiated with $\mathrm{x}$-rays compared with those irradiated with thermal neutrons. In the case of barley and also of corn, it was found that more than twice as many mutations can be produced in these plants with thermal neutrons as compared to $\mathrm{x}$-rays and still the plant will produce seeds. This means that if one is irradiating seeds or pollen in an attempt to produce a new variety, it would be more than twice as economical to use neutrons rather than $\mathbf{x}$-rays. If one is planting several acres of material, each plant of which has to be inspected carefully, a factor of two in efficiency assumes considerable importance.

As a result of this work an attempt was made to see whether it would be feasible with a relatively small amount of work to produce and isolate a given mutation. Types of mutations that would be economically desirable were to be sought. The first one was chosen in an attempt to find a mutant of a particular variety of oats which would be resistant to a particular strain of oat rust. Seed was irradiated with thermal neutrons and the first generation was grown and seed collected to make sure the character which

Figure 8. Stem rust resistance induced in the Mohawk variety of oats. The seeds were irradiated with thermal neutrons and a mutant shown on the right was found to be resistant to oat stem rust. The control plant is shown on the left. Both plants were infected with the disease but the mutant did not contract the disease. This technique is being used to create new varieties of plants with specific, predetermined characteristics.

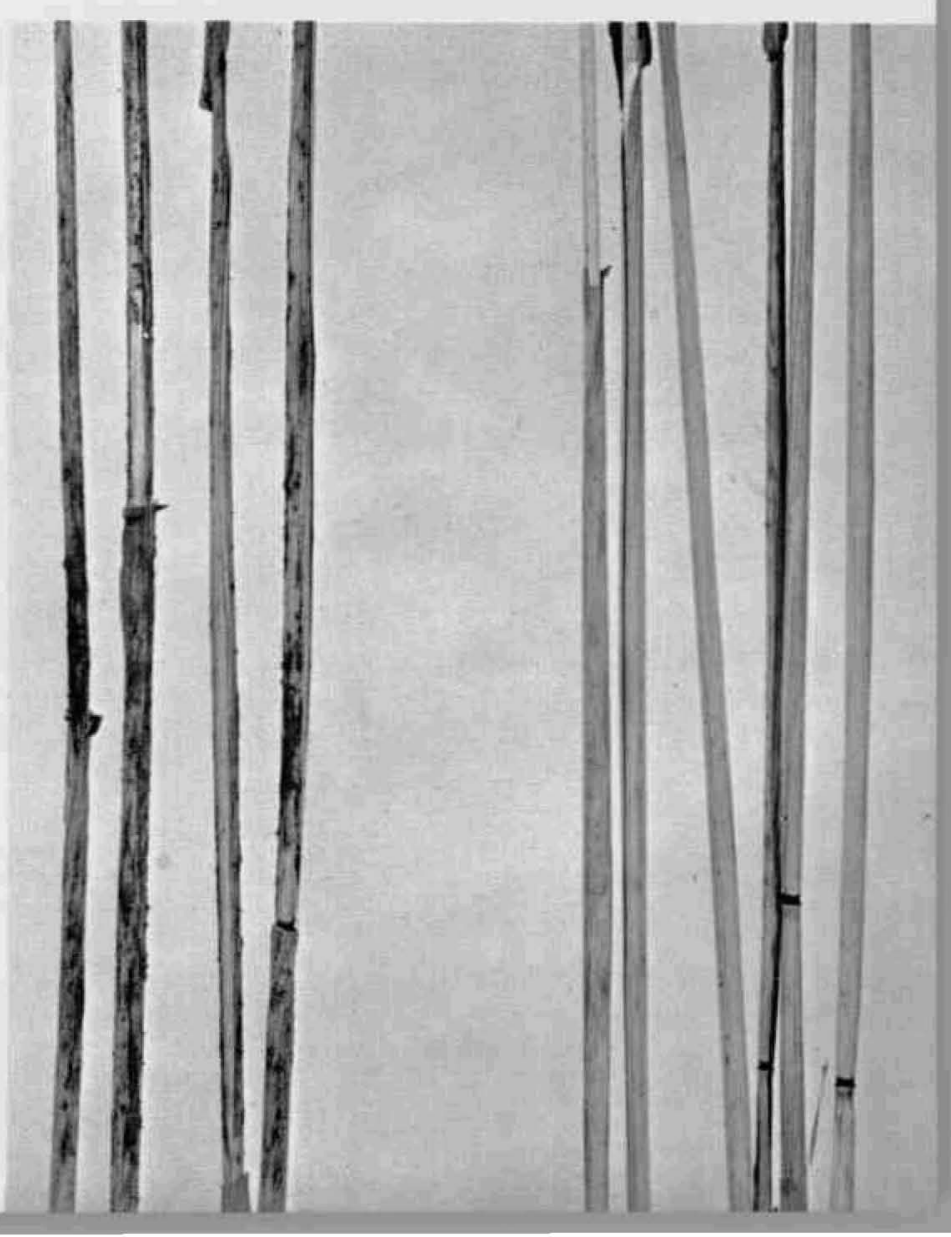


was being dealt with would be propagated genetically. The second generation was grown and when the plants were almost mature the entire field of oats was infected with this particular strain of rust. Forty-nine plants were found to be resistant (Figure 8). Seed from these resistant plants was grown again and it was found that in all cases the character bred true.

This demonstrates that it is possible in the course of about 18 months to develop a plant resistant to a particular disease but in all other respects identical to the parent variety. This might well take at least ten years by conventional breeding methods. Various races of wheat, oats and barley rust spread through the country and cause an estimated annual agricultural loss of many millions of dollars. By conventional breeding methods it has been virtually impossible to keep up with the new plant diseases but with this new technique many diseases should be brought much more nearly under control.

In connection with this work, it was noted that in the same variety some of the irradiated plants matured earlier than normal and at least one of them matured later than normal (Figure 9). This again is a demonstration of the way in which radiationinduced mutations can be used to produce agriculturally important characters in plants. Indeed, one might almost say that this is a method by which new varieties of plants can be produced to specifications.

This laboratory is cooperating with about 30 universities and experiment stations in an attempt to produce new varieties of plants by irradiation of the seed. For example, the Department is working with the Texas Experiment Station in an attempt to produce rice with a short stalk which will resist wind damage, with the University of Nebraska to produce soy beans which mature earlier, with the University of California to produce flax resistant to Fusarium wilt. The laboratory takes the responsibility for deciding on the radiation technique and dosage. This usually involves irradiating an aliquot of the seed at several different dosages, growing the seedlings in greenhouse flats, judging from this the proper radiation dosage and then administering it. The cooperating institution then grows the material and screens

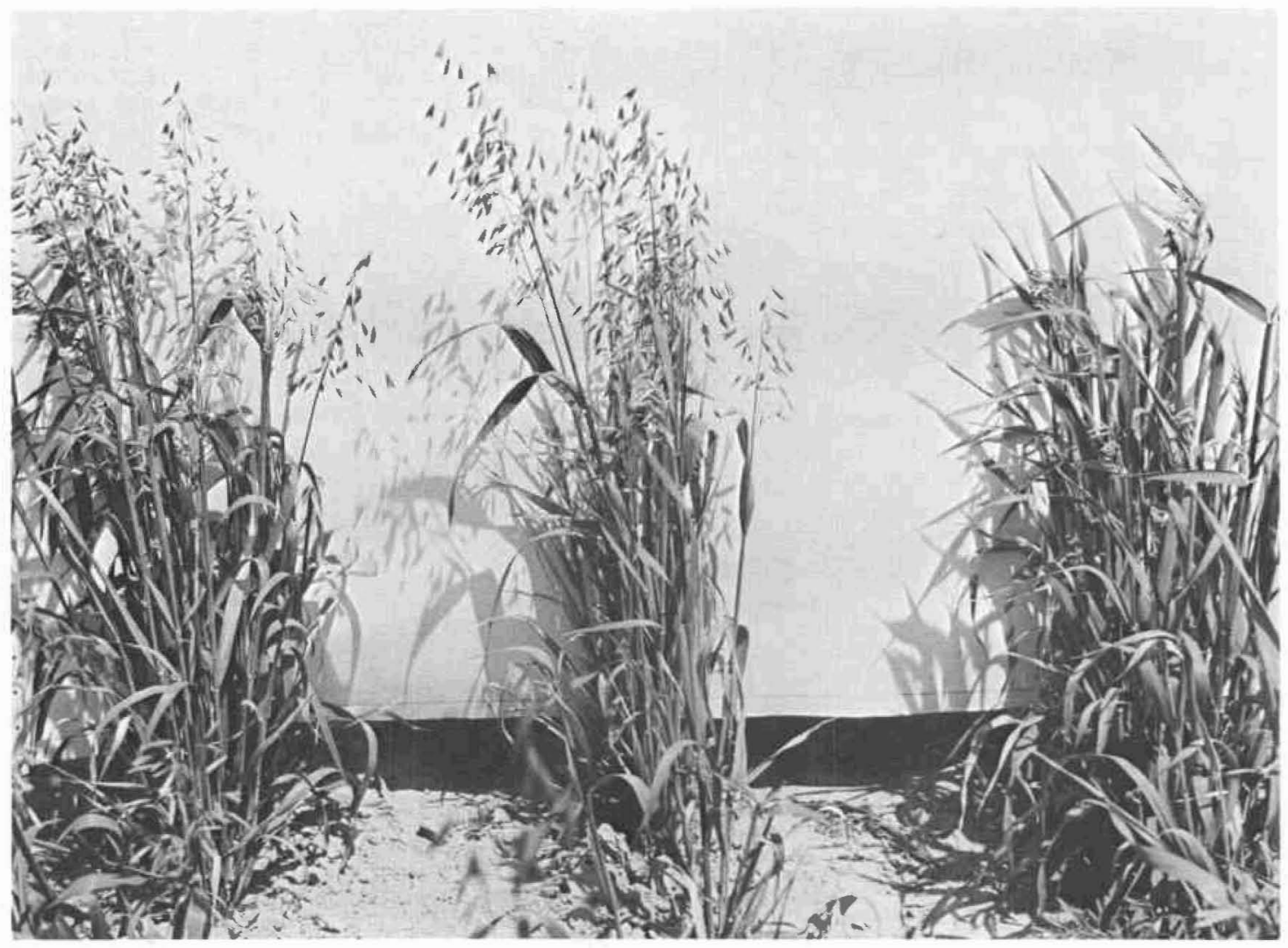

Figure 9. Thermal neutron induced mutant in oats. The control plant is shown on the right and two of the mutant plants on the left. These mutants ripen a week earlier than the parent variety but are identical in other respects. Mutants have also been segregated which ripen a week later than the parent variety. Both these mutants are agriculturally important. 


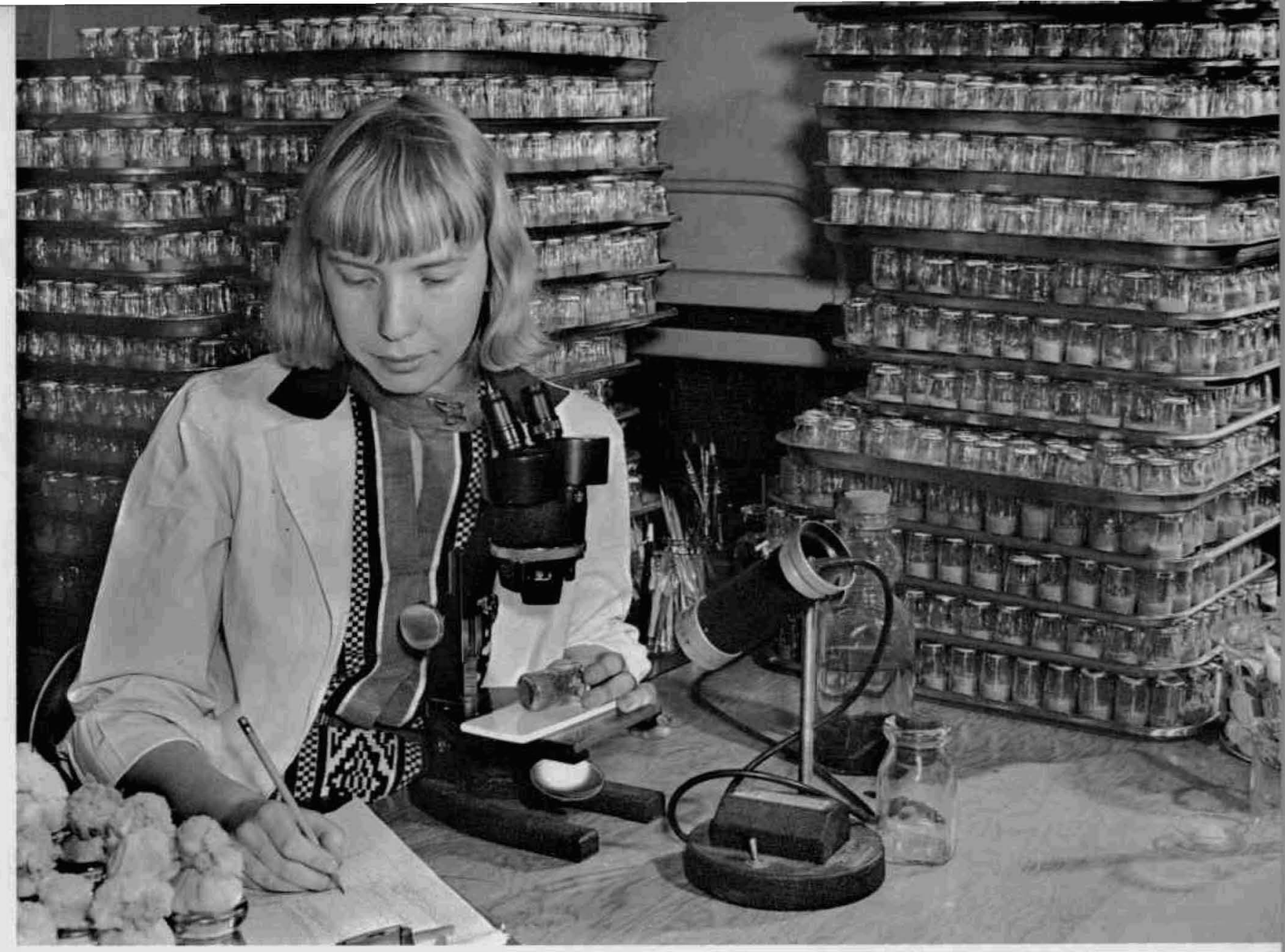

$\checkmark$

Figure 10. Photograph showing part of the Drosophile colony required for genetic experiment and the method used for scoring the heritable characters. Each small bottle contains approximately 200 flies.

it for the kinds of mutations they are interested in. Results are reported back to this laboratory.

This research, together with the somatic mutation work described in the section on Plant Physiology, is a very large program in its entirety. Although the idea of using ionizing radiations for the production of useful mutations is by no means new, this is the first time the idea has been tried on a large enough scale and with completely adequate radiation facilities to give it a satisfactory test. Present indications are that it will prove to be a very powerful technique for plant breeding.

Work is in progress on the parasitic wasp Habrobracon which can be bred in such a way that males have only half the chromosome complement of normal males. Since many people think that radiation effects are due entirely to effects on the chromosome, this material gives an opportunity to vary the chromosome complement of the cell without varying anything else. Results so far indicate that the chromosome complement of the cells is by no means the only factor which must be taken into account in estimat- ing the radiosensitivity of the cell. When treated in certain stages of development, the haploids are more sensitive than the diploids but in other stages of development the reverse is true. The work is continuing with different gases such as oxygen, nitrogen, and carbon dioxide being used to modify this effect.

Closely associated with this work is that on the fruit fly Drosopbila which has long been used as a material for study of genetics, because enormous numbers of flies can be bred easily and quickly (Figures 10 and 11). A comparison has been made between the mutagenic efficiency of $x$-rays and thermal neutrons. It is found that this efficiency is different depending upon the particular gene locus one is studying, which fact gives another indication that the mechanisms by which these two radiations produce mutations is different. Comparing the two radiations on the basis of energy dissipated in the tissue, it is found that for the most sensitive criterion, viable sex chromosome losses, thermal neutrons are 2.5 times more effective than x-rays. An extension of this work has shown that the mutagenic efficiency of beta rays 


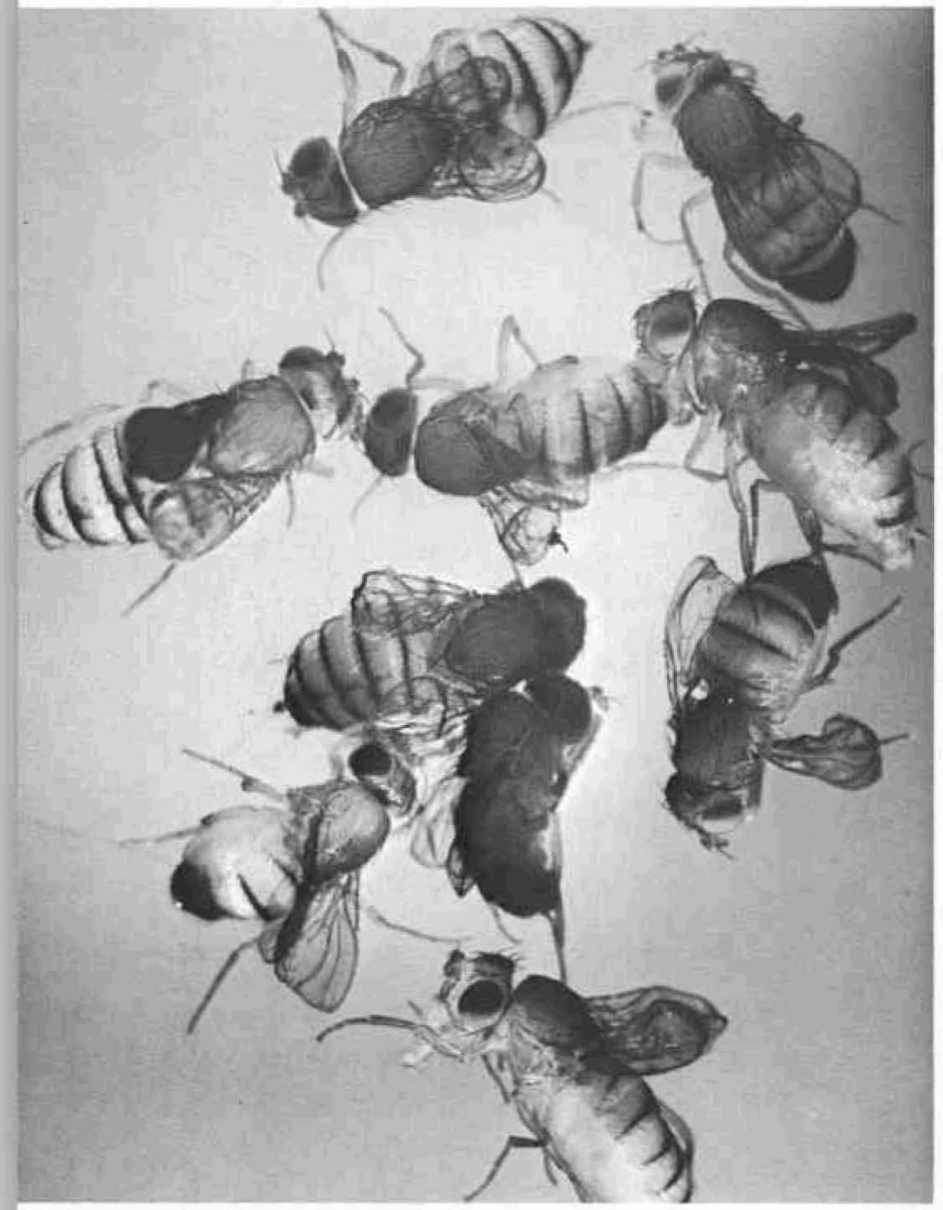

Figure 11. Mutations induced in Drosopbila by feeding radioactive phosphorus to the male prior to mating. Some of the offspring exhibited rudimentary wings as shown in the photograph and subsequent mating showed this character to be a true mutation.

is 60 percent that of $90 \mathrm{kw} \mathrm{x}$-rays, with sex-linked lethal mutations as the criterion.

\section{METABOLISM}

The work in metabolism is concerned entirely with the use of isotopic tracers for an investigation of the details of many complex biochemical reactions and the mechanisms by which these reactions are brought about. Since virtually all biochemical reactions are activated by specific enzymes, it is important to know the mechanism by which these enzymes function. By labeling specific atoms in the substrate and then analyzing the reaction products and following the position of the labeled atom, it is possible to obtain a fairly clear idea of the sequence of events in the course of a reaction. One example of this method is the hydrolysis of adenosine triphosphate in the presence of myocin. In this case it was shown that the reaction was an attack on the terminal phosphate of the adenosine triphosphate by means of an exchange between the oxygen of water and inorganic phosphate. The kinetics of a number of other biochemical reactions have been studied by labeling specific atoms in the molecule.

As a result of these studies, it has been possible to formulate a tentative theory of enzymatic action. This theory essentially implies that no basic change in mechanism occurs on going from simple organic and inorganic catalysis to the protein molecule. It can be shown that this hypothesis can lead to an explanation of stereochemical changes in enzymatic reactions and to the deduction of a generalization relating enzyme specificity to the point of cleavage in the hydrolytic reactions. A number of enzymatic reactions are being studied by the tracer technique in an effort to corroborate this theory and to give it strength with more specific examples.

Another project in this field is a biochemical study of protein and phospholipid synthesis in the mouse. The method involves feeding labeled precursors of the compound in question and, by an analysis of product and turnover rates, deducing the nature of the reaction involved. For this work it has been necessary to develop extremely sensitive counting procedures as well as very sensitive methods for identification of very small quantities of these various compounds.

In this study glucose was one of the important precursors of many of these compounds and during the past year work has been concentrated on the effect of the endocrines on glucose metabolism. Preliminary indications are that hypophysectomized dogs have about twice the normal rate of body glucose turnover. Furthermore, in normal dogs the injection of insulin causes a very rapid decline in the blood sugar levels and this is accompanied by a fall in glucose utilization rate. Also, for rats fed $\mathrm{C}^{14}$ glucose by stomach tube the normal rat uses only about half as much glucose for direct conversion to $\mathrm{CO}_{2}$ as does the adrenalectomized rat. The adrenalectomized rat cannot readily convert noncarbohydrate stores to carbohydrate. The present experiment indicates that a reverse reaction is likewise impeded in the adrenalectomized animal. No general statement can be made as yet concerning the specific mechanisms for hor- 
an attempt to elucidate the function of this substance in cells. Many people believe that the chromosomes and thus the genes are composed almost entirely, if not entirely, of nucleoproteins and if this is true then one could justifiably say that the nucleoproteins form the basis for all of life.

A study has been made of the time during cell division at which nucleic acid (DNA) is formed. DNA synthesis is measured by changes in DNA content of individual cells, these changes determined by microspectrophotometric analysis. It indicates that the synthesis occurs prior to cell division at specific periods which are not necessarily identical morphologically but which are special to each of the four divisions involved in pollen maturation. Methods have been worked out for measuring by autoradiographic techniques the incorporation of phosphorus in the DNA molecule. It has been found that phosphorus incorporation in the same material used for spectrophotometric analysis (Trillium pollen mother cells) follows closely the actual DNA synthesis.

In addition, a study has been made of the DNA content of haploid, diploid and tetraploid cells of tomatoes. It appears that this material does not strictly follow the DNA constancy rule but it is not yet clear what the exact nature of the difference is.

In connection with this work, and in collaboration with the Carnegie Institution of Washington, experiments have been under way to test the advisability of using a Vidicon tube in conjunction with television apparatus for making microspectrophotometric analyses of cells. With a special sensitive ultraviolet Vidicon tube it appears that this is perhaps the most satisfactory method for viewing material in ultraviolet, also for making ultraviolet absorption measurements. The apparatus was extended to include a visual trace on an oscillograph tube of the ultraviolet absorption as the Vidicon scanner crosses the cell. In this way it is possible to obtain a quantitative measure of the position of certain substances, notably DNA, within the individual cells (Figure 12).

By the use of radioactive tracers, a study has been under way for several years of the utilization of trace elements by plants, insects and algae. Recently this study has been concentrated on the intercellular and even intracellular localization of strontium, barium, iron and calcium in insects. It has been necessary to develop very precise radioautographic techniques for this localization. It is found that the cells of the gut wall take up surprisingly large amounts of these elements (Figure 13).

Another similar study is one on the mode of action and fate of antigens. The problem is being attacked by tracer techniques; both counting methods and autoradiographic methods are employed for the detection of the tracer. Methods have been worked out for labeling the antigen and for detecting it in the animal. With these techniques a comparison has been made of the rate at which sodium iodide, diiodotyrosine and the diphtheria toxoid disappear from the serum when injected into guinea pigs. Indications are that the antigen is not eliminated more rapidly than would be expected from normal catabolism but it is retained longer in antibody-producing tissue than in other tissue.

\section{MAMMALIAN PHYSIOLOGY}

The work in mammalian physiology lies in two general categories. The first is an attempt to discover some of the mechanisms responsible for the radiation syndrome in the mammal. The second is the use of the special facilities at this Laboratory for an elucidation of the basic mechanisms in mammalian function.

In the first of these, an important study is under way of the endocrine aspects of the radiation syndrome. A number of physiological mechanisms are being examined in normal animals and compared with those in animals from which various endocrine glands have been removed. Specifically, it has been found that when hypophysectomized rats are subjected to radiation they do not show the changes in glycogen content of the liver and cholesterol content of the adrenal gland which are characteristic of the intact rat. It is thus clear that normally these effects of irradiation are primarily a pituitary response and one is tempted to generalize by saying that in this respect radiation merely acts as a form of general stress. Furthermore, by direct irradiation of the pituitary gland alone in the rat, it is possible to observe many of the effects typical of total-body irradiation such as fall in the white count, increased adrenal ascorbic acid, decreased testes weight, etc. This lends additional support to the idea that many of the responses observed after whole-body irradiation are mediated in part at least by the pituitary.

These studies have recently been extended to include the effect of fasting upon the reactions. After a 5-day fast, the rise in liver glycogen is greatly re- 
duced but the drop in ascorbic acid and total cholesterol levels of the adrenal gland seem to be little affected by the fasting. The conclusion is that the ability of the pituitary gland to increase its output of ACTH is not decreased by extensive fasting but that the ability of the liver to respond to a stimulus is decreased markedly.

These, and other measurable physiological quantities, are being investigated from the point of view of neutron irradiation. Since marked changes have been found between $\mathrm{x}$-rays and neutron irradiation in plants, it seems reasonable to suppose there might be considerable difference between these two radiations as far as animals are concerned. It is too early to be able to predict the outcome of this work.

Another closely related study originated from the finding that radiothyroidectomized mice will live normally but if they are also given a sublethal dose of $\mathrm{x}$-radiation when quite young they develop pituitary tumors at the age of about 15 months. Apparently, the relation between the time of radiothyroidectomy and irradiation is not very critical. Furthermore, it has been found that radiothyroidectomy followed by either head or body $\mathbf{x}$-irradiation will produce pituitary tumors. Thus the radiation does not necessarily have to act upon the pituitary itself but acts indirectly as a carcinogenic agent.

It has also been found that if young adult male mice are castrated and then given a relatively large dose of radiation they develop a pituitary tumor at about 14 months of age. The tumor is comparable to that which follows a radiothyroidectomy and irradiation except that different cells in the anterior lobe of the pituitary are involved. This again gives radiation an essential but nonspecific role in tumor production.

The mechanism by which radiation causes these changes in the mammal is very poorly understood but one theory is that there is a toxic substance formed in tissues by the radiation which enters the blood stream and causes the generalized radiation responses. In the past, attempts to find this toxic agent have proved futile. The attempts have usually been made by injecting blood or serum from an irradiated animal into a normal animal and looking for radiation effects in the recipient animal. No effects have been observed. Recently, adrenalectomized mice were injected intravenously with serum from irradiated adrenalectomized rats. This will cause the death of these mice when given in amounts as small as $0.2 \mathrm{cc}$, and when taken from the irradiated rat 24 hours post-irradiation. The chemical nature of this toxic factor is not as yet known except that it is very labile, being destroyed if the serum is allowed to stand 30 minutes at room temperature.

Part of the long-term radiation syndrome is believed to be the result of a deficiency of the circulation which is caused by the action of the radiations on blood capillaries. A study under highly controlled conditions has been in progress for several years in an attempt to define the factors responsible for the exchange of substances between cells and the circulation. An apparatus has been perfected which meas. ures the capillary permeability of the blood-perfused hind limb of the cat with considerable accuracy. It is found that substances which are lipid-soluble pass through the capillary wall with extreme rapidity compared with molecules of comparable size which are not lipid-soluble. The permeability of these lipidsoluble substances is not influenced by molecular size or shape but is dependent entirely upon lipid solubil. ity. Thus there seem to be two mechanisms by which substances penetrate the capillary wall: one is a diffusion through pores and the other is a solubility of the lipid in the capillary membrane. For diffusion of substances through the pores, the rate of penetration is directly proportional to the size of the molecule.

As a result of these studies, a theory of filtration and diffusion through porous membranes has been developed and verified by direct measurements of the diffusion and filtration of tritium-labeled water and aqueous solutions of various compounds. The experiments lend considerable support to the theory and it is now thought that the theory for the diffusion of substances through porous membranes is on a reasonably firm basis.

A number of diseases of animals which in the past have been thought to be due to infectious disease are now thought to be hereditary in nature. Some of these are being investigated now, the first of these being retinal atrophy in dogs and the second cerebellar ataxia in cats. These studies are long-term studies and no results can be expected for a year or two.

\section{BIOPHYSICS}

The work in biophysics is concerned with an application of the ideas and techniques of physics to biology. Thus, a good deal of the work is concerned with the design and construction of special equipment. Perhaps the most important activity in this 
connection is the design, construction and calibration of the neutron irradiation facility (Figure 14). This facility, which now can be used for thermal neutrons or fast neutrons, is complete but the calibration is not yet perfect. Preliminary biological data have been obtained which indicate this will be a very valuable tool for research, not only in radiobiology but in a number of other fields of biology as well. A good deal of interest has been expressed throughout the United States and in some foreign countries in the thermal column as a practical tool for production of genetic change and in cooperating with this Laboratory in the use of the column for studies of a fundamental radiobiological nature. As a result of this interest, the facility is now in demand 24 hours a day, 7 days a week (except for pile shutdowns).

In order to understand the action of radiation on biological systems, it is first necessary to know, from a physical point of view, something of the nature of the interactions between radiation and molecules of biological importance. Very little is known about even the most fundamental reactions in radiation chemistry. In the Chemistry Department, work is in progress on the reaction between radiation and simple molecules such as water. In the Biology Department, the problem is being attacked at the level of the amino acid and simple proteins. One of the most widely used molecules is fibrinogen. When subjected to irradiation it is found that there are many smaller molecules and some larger ones, which suggests that there are two types of reaction operative: one a destructive reaction and one a condensation reaction. Ultracentrifuge and electrophoresis measurements indicate that the size and shape of a molecule as well as its reaction to radiation vary enormously depending upon the other constituents of the solution, such

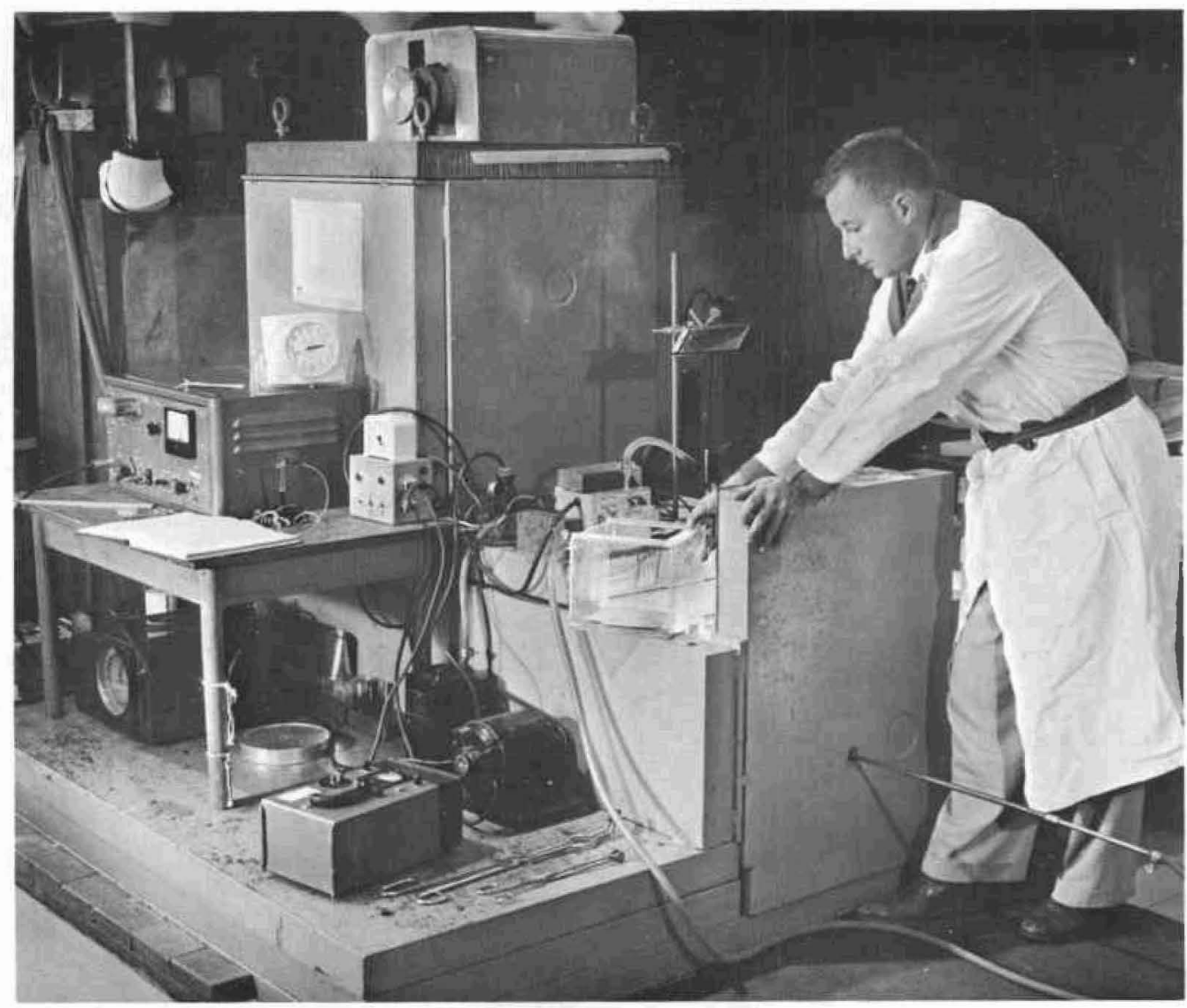

Figure 14. Photograph of the neutron facility on the top of the Brookhaven reactor. A box containing biological material is being placed in the tunnel which leads to the elevator which will lower the box into the thermal column. For fast neutron irradiation a uranium plate is placed at the bottom of the exposure box and lowered into the thermal column. The thermal neutron fission of uranium creates fast neutrons which then give a predominant fast neutron exposure to the material placed in the top part of the exposure box. 
as urea, and $\mathrm{pH}$. In order to characterize the molecular size and shape completely, it is necessary to perform accurate diffusion measurements, and recently a good deal of effort has gone into the perfection of a method for making these measurements.

Another phase of this same work is a study of the action of radiation on bacteriophage. Bacteriophage can be considered as a very large molecule and is known to behave in many respects as the simplest biological system. The size and shape of this molecule is known from other studies so by its use the radius of action of ionizing radiation can be determined. It turns out that the relation between the radiosensitive volume of the virus and the total volume is not a simple relationship as indicated by the "hit theory" of radiation damage but depends upon a number of complex factors. It appears that there are a number of relatively independent "sensitive volumes" in a virus particle. For example, one may by irradiation inactivate the virus as far as its antigenic properties are concerned but leave it capable of infecting a bacterial cell. Several such "sensitive volumes" are being worked out with a view toward physically characterizing the virus particle as being made up of a number of parts physically separate, each part having a relatively independent function to perform.

Another quite independent study is an attempt to elucidate the mechanism by which reduplication takes place in the nucleus of a cell during cell division. The tracer technique offers a possibility of shedding some light on this important problem. The attempt has been made to label isotopically some of the nuclear material in a simple cell and follow this through several generations with the autoradiographic technique. If successful, the method should show whether there is a template mechanism operative for the production of new chromosomes or whether there is a general mixing of chromosomal material at the time of cell division which, by some unknown mechanism, reduplicates the chromosomal material. Very sensitive autoradiographic methods have been worked out which effectively give a resolution for this technique of less than 1 micron. Present indications are that after a cell division some of the original nucleoprotein goes with each of the daughter cells. However, it is now believed that even in the simple cell used, $E$. coli, there are several independent chromosomes, so this cannot be taken as positive evidence that the template theory is incorrect.

\section{MEDICINE}

The major work in the Medical Department during the past year has continued along the outlines listed previously. Broadly, the research is oriented toward the use of the special facilities provided at Brookhaven and is directed toward: 1) the use of isotopes for tracer techniques, 2) therapy with shortlived radioisotopes, 3 ) study of radiation effects, and 4) neutron capture therapy. This last is properly a subdivision of 2 above, but because of the utilization of special techniques and facilities, it has been classed separately. Details of the programs will be discussed under these headings.

The year witnessed the Medical Department's entrance into the summer program for visiting scientists and students, but because of space limitations the number of visitors was and will continue to be sharply limited. The collaborative work was continued with a research team from the University of Iowa College of Medicine, a group from St. Albans Naval Hospital, St. Albans, N. Y., and members of the Memorial Hospital and Sloan-Kettering Institute, New York City. The investigators from Iowa, in association with the Division of Biophysics, extended their observations on xenon, pursuant to theoretical considerations of its anesthetic properties. The other work will be noted later.

Plans for a new medical center at BNL are in progress. The floor plans which were originally drawn up by the Architectural Planning Division are now being reviewed by an outside firm of architects for final details and cost estimates. The design of a special medical reactor is undergoing study by the Advance Technology Corporation in conjunction with the Reactor Operations Division and the Nuclear Engineering Department at BNL.

\section{THE USE OF ISOTOPES FOR TRACER TECHNIQUES}

Members in all the research divisions used isotopes for investigative purposes during the year. The close association between work in the laboratory and that with patients continued with reciprocal stimulation to both clinical and nonclinical investigators.

In the Division of Physiology, distribution and degradation studies employing $\mathrm{I}^{131}$ human serum albumin have been carried out on a group of thyroid carcinoma patients rendered myxedematous by surgical thyroidectomy, therapeutic radioiodine, thyroid 
blocking drugs, or by a combination of these agents. The studies have been repeated when the patients have returned to the euthvroid state after administration of desiccated thyroid or triiodothyronine. In selected cases the metabolic status was altered during the distribution study. The results of these studies indicate a 30 percent increase in the total exchangeable albumin in the myxedematous state, which is due to an increase of approximately 70 percent in the extra. vascular exchangeable albumin fraction, with no significant change in the total plasma albumin. Plasma concentration curves of the indinated albumin, compared with the body retention curves followed up to 36 days, suggest the presence of an extravascular compartment with an exceedingly slow turnover rate. The existence of this compartment was supported by the results of daily in vivo counts over the liver and thigh in selected patients. The degradation rate of iodoalbumin was also shown to be markedly increased by thyroid hormone. The characteristics of the plasma concentration and body retention curves favor the plasma, or a compartment in rapid equilibrium with plasma, as the site of degradation for iodoalbumin.

Tracer studies directed toward the elucidation of the mechanism(s) of ion transport across the mem-

Figure 1. Study of smooth muscle with tissue slice technique by means of the Warburg apparatus.

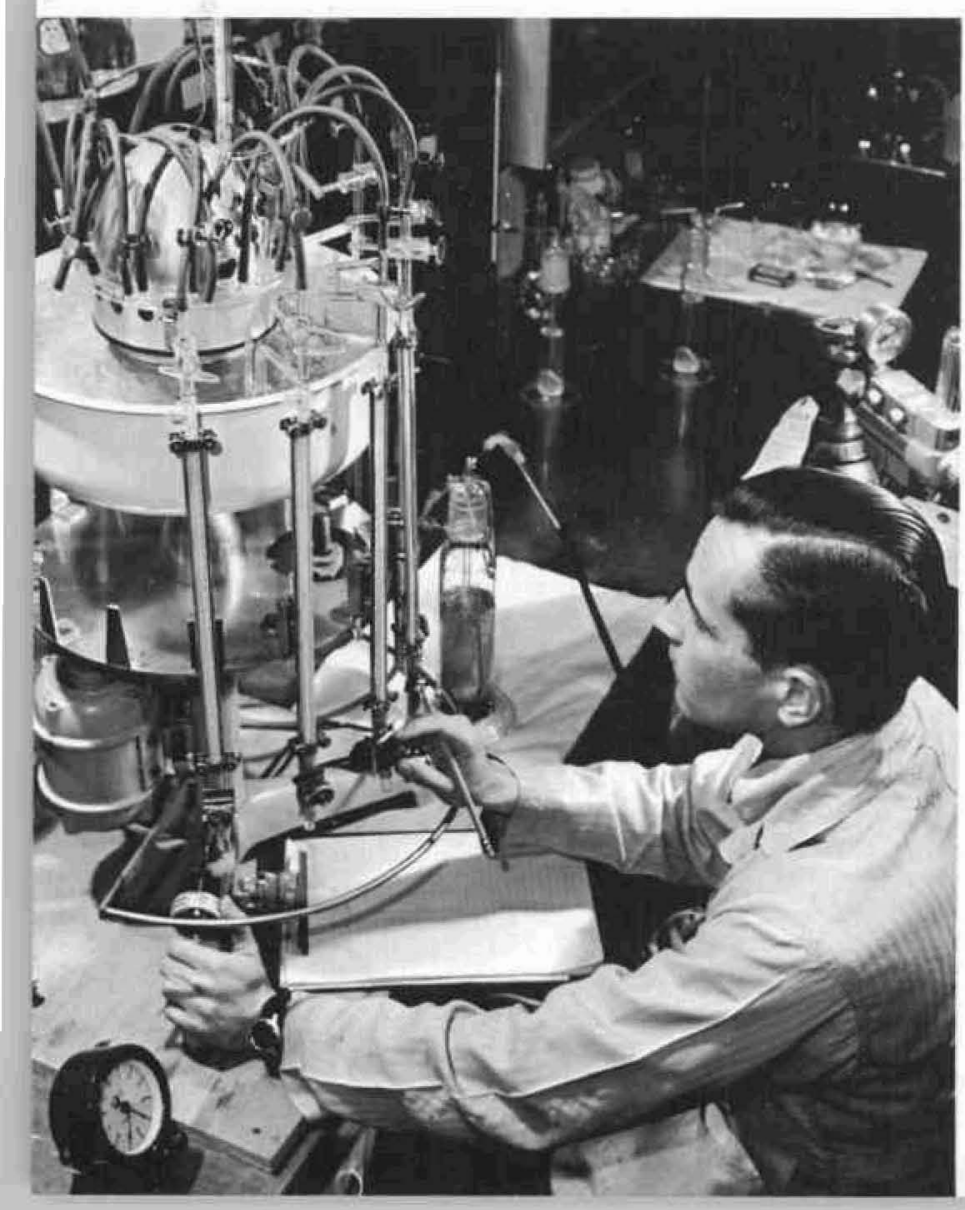

branes of living cells have continued. The effects of $\mathrm{pH}$ changes and of altered cation composition on the rates of movement of sodium and potassium across the red cell membrane have been investigated. At reduced $\mathrm{pH}$ levels a marked diminution of potas. sium influx and sodium outflux has been observed and found to be reversible when the $\mathrm{pH}$ was raised again. The mechanism by which these $\mathrm{pH}$ effects are induced is being explored.

Further work has been done to explore the mechanism by which sodium restriction effects a lowering of blood pressure in hypertensive individuals. A series of 11 patients with essential hypertension was studied before and after $\mathrm{Na}$ intake was restricted to approximately $6 \mathrm{mFq} /$ day. Changes in blood pressure were not correlated with changes in total exchangeable sodium after restriction of this element in the diet. Measurements of total exchangeable $\mathrm{K}$ failed to reveal correlation with either change in total exchangeable $\mathrm{Na}$ or fall in blood pressure. Before and after marked $\mathrm{NaCl}$ restriction, another series of 7 patients was studied recently with $\mathrm{Na}^{24}$ and $\mathrm{Br}^{82}$; by means of these isotopes the effects on total exchangeable $\mathrm{Na}$ and $\mathrm{Cl}$ were studied for correlation with blood pressure decline. In view of the limited intake of $\mathrm{Na}$ and $\mathrm{Cl}$ which these patients have, the question of long term balance has often arisen, and in such instances, skir losses could be important. This was studied directly by measuring the total skin losses of $\mathrm{Na}, \mathrm{Cl}$, and $\mathrm{K}$ for two periods of 7 and 3 days in patients who had been on $\mathrm{NaCl}$ restriction for 26 to 150 days. The measured losses of all three electrolytes proved to be significantly lower 'than the values previously recorded, and of such an order that except in patients with excessive renal losses net balance could be maintained.

As part of the hypertension research program, studies on smooth muscle have been undertaken since the lowest common denominator to which hypertension can be reduced is an abnormal constriction of the smooth muscle cells of the arteriolar bed. The present studies are concerned with the metabol. ism of smooth muscle tissue with the objective of setting up an $2 n$ vitro system in which various alterations in metabolism can be studied relative to their effects on the ability of smooth muscle to contract or relax. Classical tissue slice technique with incubation in appropriately buffered solutions in the Warburg apparatus is being used. Interest has centered largely on oxygen consumption and $\mathrm{Na}$ or $\mathrm{K}$ movement in and out of the muscle slices. 


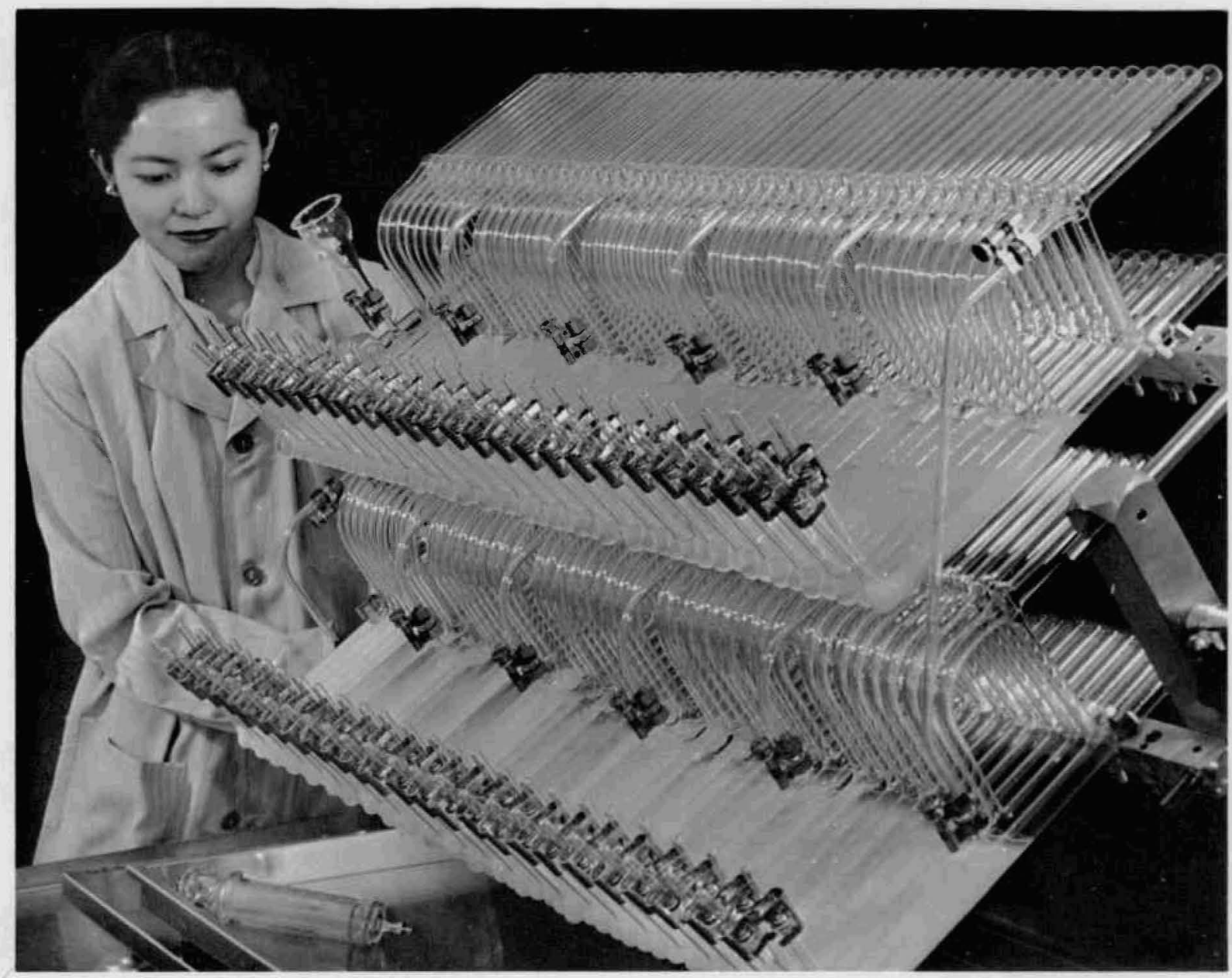

Figure 2. Craig counter-current distribution apparatus being used for fractionation of histones in chromosomal protein.

Measurements of cardiac output, circulation time, and pulmonary capillary volume have been made in dogs, by means of continuous flow counters and either $\mathrm{K}^{42}$ or $\mathrm{Na}^{21}$ :

In the Division of Biochemistry, studies of collagen synthesis with $\mathrm{C}^{1+4}$ have established the fact that hydroxylysine is formed from lysine. Studies of its basic character were undertaken since collagen is the principal extracellular protein of tissue, and as such, it has a virtually ubiquitous distribution and importance in a wide variety of disease processes. Hydroxylysine is one of the two unique amino acids in collagen; studies on the other, hydroxyproline, are under way. Other studies with $\mathrm{C}^{14}$ on the intermediary metabolism of protein in rats revealed that 3-hydroxyanthranilic acid was converted to $\mathrm{N}$. methyl nicotinamide, which suggests that the former compound is an intermediate in the synthesis of niacin from tryptophane.
For many years there have been two theories or hypotheses to explain the phenomenon of the passage of fat from the lumen of the intestine through the intestinal wall and thence to the rest of the body. One hypothesis maintained that fat was broken down completely to glycerol and fatty acids during absorption, whereas, according to the second theory, complete splitting was unnecessary and unsplit fat could be absorbed provided there was sufficient emulsification in the intestine. Much evidence has accumulated in favor of each theory, but many published data have failed to support certain details of each of the hypotheses.

A study of the mechanism of intestinal fat absorption was undertaken in order to help clarify some of the problems of this complicated and manyphased process. $C^{14}$-glycerol-labeled fat was utilized in this work, and its use enabled new observations to be made which were heretofore unobtainable with 
nonisotopic fat or with fat labeled in the fatty acid moiety. From the results it appears that neither of the cited theories operates to the exclusion of the other, but that physiological absorption is, so to speak, a compromise. For instance, four hours after rats were given $\mathrm{C}^{14}$-glycerol-labeled triolein, 90 to 97 percent had been absorbed from the gastrointestinal tract, but only 50 percent of the fat had been hydrolyzed. Thus nonhydrolyzed liquid must have been absorbed in significent quantities. Complete lipolysis was found to occur within the gut lumen as well as in the tissues, for free radioactive glycerol could be isolated from the lumen.

A number of studies on thyroid physiology were made in the Division of Pathology. A rapid and simple method for detection of TSH was developed, in which the increased thyroidal accumulation of $\mathrm{I}^{131}$ in young chicks was utilized. In confirmation of Hamilton's work, the rat thyroid gland was shown to accumulate selectively the halogen-like element astatine, but under similar experimental conditions, less astatine than $\mathrm{I}^{131}$ was accumulated. Thiouracil was found to enhance the accumulation of astatine.

\section{USE OF INTERNALLY ADMINISTERED RADIOACTIVE ISOTOPES IN THERAPY}

In collaboration with members of the Memorial Hospital and the Sloan-Kettering Institute, New York City, the study and treatment of patients with metastatic thyroid cancer has continued. One of these patients, who had received a total dose of $794 \mathrm{mc}$ of $\mathrm{I}^{131}$ over a period of three years and eight months, was found to have early myelogenous leukemia at autopsy. Although no unequivocal relationship can be established between the leukemia and the radiation in this case, it emphasizes the necessity for limiting heavy radiation to patients with serious diseases.

As a service to the surrounding community, selected patients with thyrotoxicosis have been treated with $I^{131}$. Similarly, during the past year 12 patients with polycythemia vera were referred here for consideration of treatment with $\mathrm{P}^{32}$. Because these diseases have not been under primary investigation, the patients usually have been treated on an outpatient basis.

In the study of the distribution of radiomanganese in rat tissues, it was confirmed that high concentrations of $\mathrm{Mn}$ occurred in liver and pancreas. Calculations indicated that if an isotope with a suit- ably short half-life were chosen, heavy radiation could be administered to these organs without undue effect on other tissues. With these considerations in mind, it seemed reasonable and desirable to attempt therapy on patients suffering from cancer in one of these two sites. $\mathrm{Mn}^{56}$, with a 2.6-hr half-life, was selected for use on two patients with carcinoma of the liver. One carcinoma was primary in that organ, and the other was secondary to a large bowel cancer. Both patients ultimately died, but the temporary results warrant further investigation.

Gallium is known to be one of the many cations picked up by skeletal bone. In collaborative studies with a group from the Radioisotopes Unit, St. Albans Naval Hospital, St. Albans, N. Y., during the past year, the bone-seeking properties of the isotope $\mathrm{Ga}^{72}$ have been used to treat metastatic bone cancer. In no instance has there been unequivocal evidence of regression of lesions, but of the ten patients so treated, two have had definite relief of pain which up to the time of writing had lasted two and four months, respectively. Some interesting and reversible delayed depression of the hematopoietic system has appeared in several patients and is presently under further study.

\section{RADIATION EFFECTS}

In the Division of Microbiology, several problems have been explored during the past year in the study of the effect of ionizing radiation upon the immune mechanism. Evidence has been obtained which shows that transplants of thymus and Peyer's patch tissue taken from immunized donor mice produce antibody for tetanus toxoid while growing in the anterior chamber of the eyes of cobalt- 60 gamma-irradiated mice. The specific anamnestic response is used to determine the ability of the transplanted tissue to produce antitoxin to tetanus toxoid.

Whole-body gamma radiation (50 to $650 \mathrm{rep}$ ) of mice previously sensitized with alum-precipitated tetanus toxoid greatly increases the severity of anaphylactic shock when challenged with fluid tetanus toxoid seven days post-radiation. The enhancing effect of ionizing radiation upon the anaphylactic response was not of an immediate nature, but appeared during a two to seven-day post-radiation period. Passive and reversed passive anaphylaxis were more severe in seven-day post-irradiated mice passively sensitized with homologous antibody. Irradiated mice sensitized 


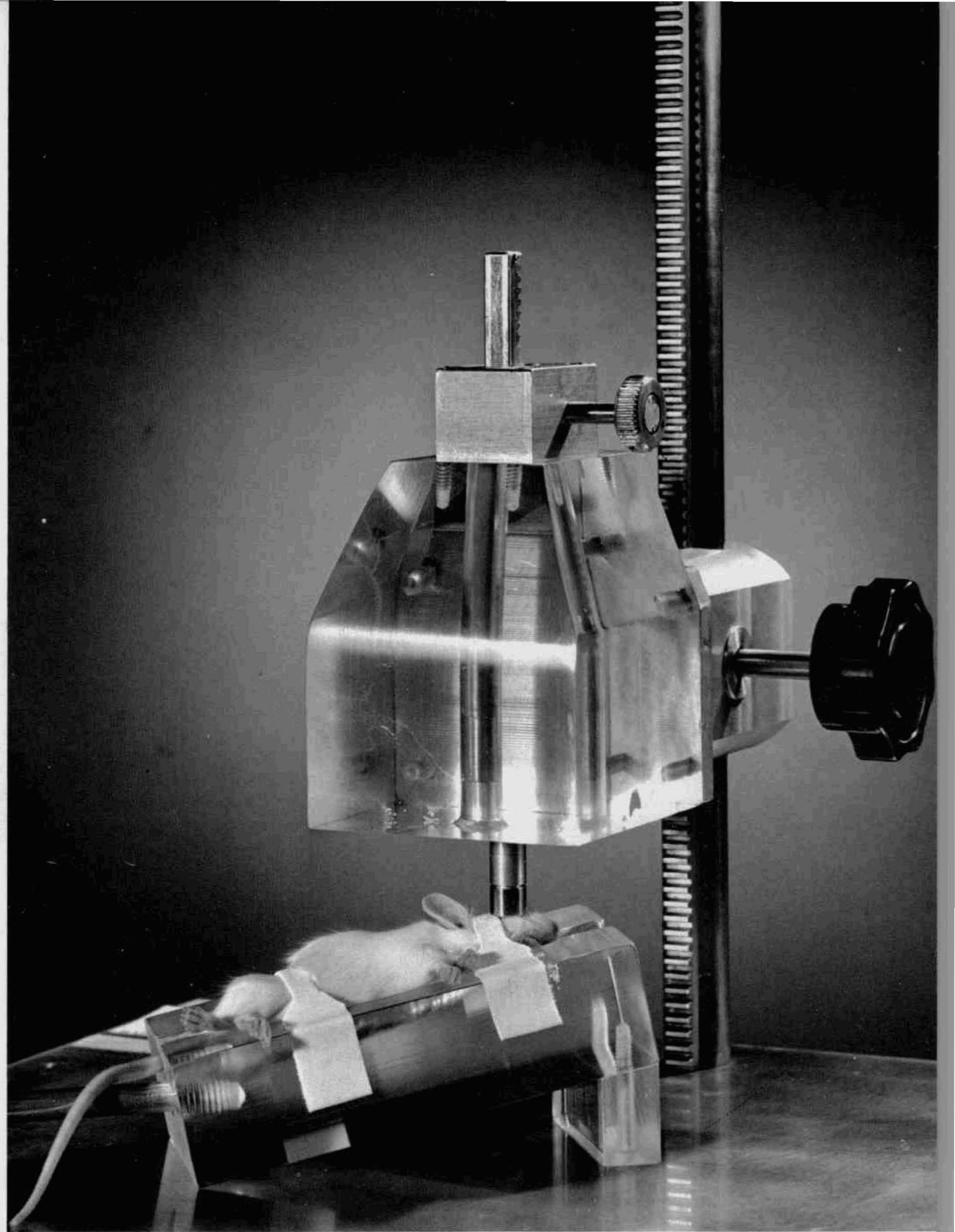

Figure 3. Thallium-204 source in use as a means of studying the sensitivity of tissues to pure beta radiations. 
with antibovine albumin rabbit serum also demonstrated increased sensitivity to passive anaphylaxis when challenged one day later with bovine albumin antigen. The antihistaminic agents, Thephorin, Benadryl and Pyronil, afforded almost complete protection from fatal anaphylaxis in irradiated mice. No significant difference was found in the sensitivity of irradiated and nonirradiated mice to intravenous injection of histamine diphosphate. This may indicate that the antihistamines do not function in their relationship to histamine in mouse anaphylaxis. Their protective action against fatal mouse anaphylaxis may be affected by virtue of some other pharmacologic property of the antihistaminic substances. This work is being extended in a study of ionizing radiation effect upon anaphylaxis in guinea pigs.

Cortisone treatment of mice immunized with active infections of Trichinella spiralis and challenged with Trichinella larvae produced a significant breakdown of their acquired immunity to reinfection. The depressing effect of cortisone upon acquired immunity to Trichinella infections was similar to that observed with sublethal gamma radiation of immunized mice. Daily administration of ACTH to immunized and challenged mice effected a slight but not significant increase in mortality over that observed in untreated control animals.

A thallium-204 beta radiation source has been developed to study radiosensitivity of tissues. The source was designed and constructed by Powell Richards of the Nuclear Engineering Department at BNL.

A program of study relative to the effect of garnma radiation on microorganisms is in progress. A smooth strain of Diplococcus pneumoniae has been used throughout most of this work because this organism possesses some distinct biochemical and physical properties which should serve as convenient indicators of radiation effects. A cobalt-60 source providing a gamma ray intensity of the order of $8 \times 10^{5}$ $\mathrm{r} / \mathrm{hr}$ has been used.

Although the bacterial cells readily lose their capacity for reproduction after exposure to ionizing radiation, it was found that suspensions of irradiated cells behaved like nonirradiated cells with respect to the reduction of triphenyltetrazolium chloride. In the presence of glucose and phosphate, the tetrazolium salt was readily reduced to formazan and no significant difference between the dehydrogenase activity of irradiated and nonirradiated cells could be demon- strated. With other techniques, it was found that glucose was actively metabolized by pneumococci which had been exposed to radiation doses that inhibit multiplication of 99.9 percent of the cells. Biologically active desoxyribonucleic acid (DNA) has been prepared from gamma-irradiated type III pneumococci. The DNA isolated from cells exposed to 100,000 and 150,000 rep possessed transforming activity of the same order of magnitude as the DNA prepared from nonirradiated cells. Exposure of highly virulent type III organisms to irradiation resulted in a loss of the invasive properties.

The Division of Pathology has continued the previously inaugurated long term studies on the radiation effects of graded doses ( 2 to $400 \mu \mathrm{C}$ ) of $\mathrm{I}^{131}$ in rats. No gross thyroid neoplasms have been discovered; histological preparations of the thyroids in the treated animals are presently undergoing detailed examination.

\section{NEUTRON CAPTURE THERAPY}

Some of the initial phases of this program have been discussed in detail in preceding annual reports. During the year just passed the medical facility in the top of the Brookhaven reactor was redesigned. This was undertaken primarily to provide a facility which would render unnecessary the requirement of bringing the reactor to full power with great speed. To avoid this hazard and also to increase the availability of this medical facility a shutter was designed which when closed provides adequate shielding on top of the reactor with a relatively light steel plate over the facility. Now it is no longer necessary to remove the heavy shielding blocks to use the medical facility, reactor operation can continue at constant power during medical runs, and when escape of neutrons from the medical facility with the shutter open poses no hazards to personnel of this and other departments, experiments can be carried out at times other than when the reactor is shut down and the area of the reactor vacated by other personnel. It is hoped that with the present shutter it will be possible to expedite many experiments which were previously planned but for which satisfactory operating arrangements could not be developed. Although the present facility is a marked improvement over the original highly experimental facility, it leaves much to be desired in the way of opportunities for more rapidly developing this phase of the Medical Department program. 
Extensive instrumentation and phantom tests together with measurements made during recent patient treatments have shown the flux at the center of the port now to be of the order of magnitude of $3 X$ $10^{9}$ thermal neutrons $/ \mathrm{cm}^{2} / \mathrm{sec}$. This is an increase of over ten times the thermal neutron flux previously available at this facility. The increased flux adds a great deal to the usefulness of this facility for the present program. On the best present estimates another tenfold increase of flux would be required for a really adequate treatment unit. The fast neutron flux has been kept at about $1 / 10,000$ of the thermal neutron flux, which should provide an adequate margin of safety in the experiments presently proposed. Gamma ray contamination has been reduced to a very low quantity, of the order of $100 \mathrm{r} / \mathrm{hr}$.

A new series of therapeutic studies has been started which makes use of neutron capture therapy. The procedure is still being confined to patients with proven malignant brain tumors of the type called glioblastoma multiforme. Four patients have been so treated in the current series but results cannot be assessed at this early date. Treatments on all four will be repeated as indicated. Other aspects of this program have involved irradiation of colonies of mice to determine biological effects of this type of radiation and radioautographic studies in boron-containing tissue slices exposed in contact with nuclear track emulsions. The purely physical instrumentation experiments include new fission neutron detectors and tissue equivalent ionization chambers of the pressure extrapolation type to determine actual tissue dose contributions from the several components of the mixed radiation beam and the many induced radiations. A program of biological titration of the reactor facility has been started in which both small animal and microorganism technics are used.

The Division of Pathology has completed an extensive histological study of brains obtained at autopsy from patients subjected to this type of therapy in the original series of experiments carried out from February 1951 through November 1952. In three of the eight patients studied at autopsy, changes in the tumor appear to be sufficient to indicate response to the radiation. Although conclusions cannot be drawn on the basis of these patients because of the tendency of spontaneous degenerative changes which occur in glioblastoma multiforme, the general impression obtained from the study is that radiation effects exist but that the dose was marginal. This is in agreement with conclusions drawn on other bases which led us to develop the facility so as to provide a higher thermal neutron flux.

\section{METHODOLOGY}

The development of new methods or the modification of existing ones has been necessary for the basic research program.

Two modified micro chambers have been developed for the Van Slyke-Neill manometric gas apparatus. These chambers should prove useful in the determination of oxygen, carbon dioxide, and plasma amino acids in blood. The gas volume may be read at $0.05,0.1$, or $0.2 \mathrm{ml}$. The quantity of blood required for the determination of oxygen or carbon dioxide has been reduced from $1 \mathrm{ml}$ to $0.05 \mathrm{ml}$.

The highly sensitive gas phase proportional counting tube devised by Van Slyke et al has been modified to make the instrument available for laboratories not equipped with power supplies delivering more than 3,000 volts. By reduction of the required voltage from 3,800 to $2,100 \mathrm{v}$, the use of the tube in $\mathrm{C}^{14} \mathrm{O}_{2}$ counting should become more general.

A modification in the photometric determination of calcium mentioned in the last annual report allows a concomitant determination of magnesium. Conditions appropriate for analysis of these ions in biological fluids have been worked out. A revision of the original Van Slyke manometric method for $\mathrm{Ca}$ has been made by which both accuracy and sensitivity have been increased.

A specific and simple procedure for the accurate determination of acetic acid in the presence of labile acetates has been developed. This has made possible the direct assay of acetylcholine esterases in tracer concentrations since the method is sensitive to approximately $0.2 \mu \mathrm{M}$ acetic acid $/ \mathrm{ml}$. The flexibility of the method is such that irradiation and other effects can be studied under a variety of conditions. The limitations imposed by previous methods in terms of amounts of substrate, large enzyme concentrations, complicated apparatus, and indirect end points of the assay have been eliminated.

Preliminary tests had revealed that the sensitivity of monoamine oxidase to $\beta$ and $\gamma$ irradiation was increased when the enzyme was rendered soluble. Solubilization of the enzyme had not previously been achieved in satisfactory fashion, but after some trials it was accomplished by means of isoctylphenoxypoly- 


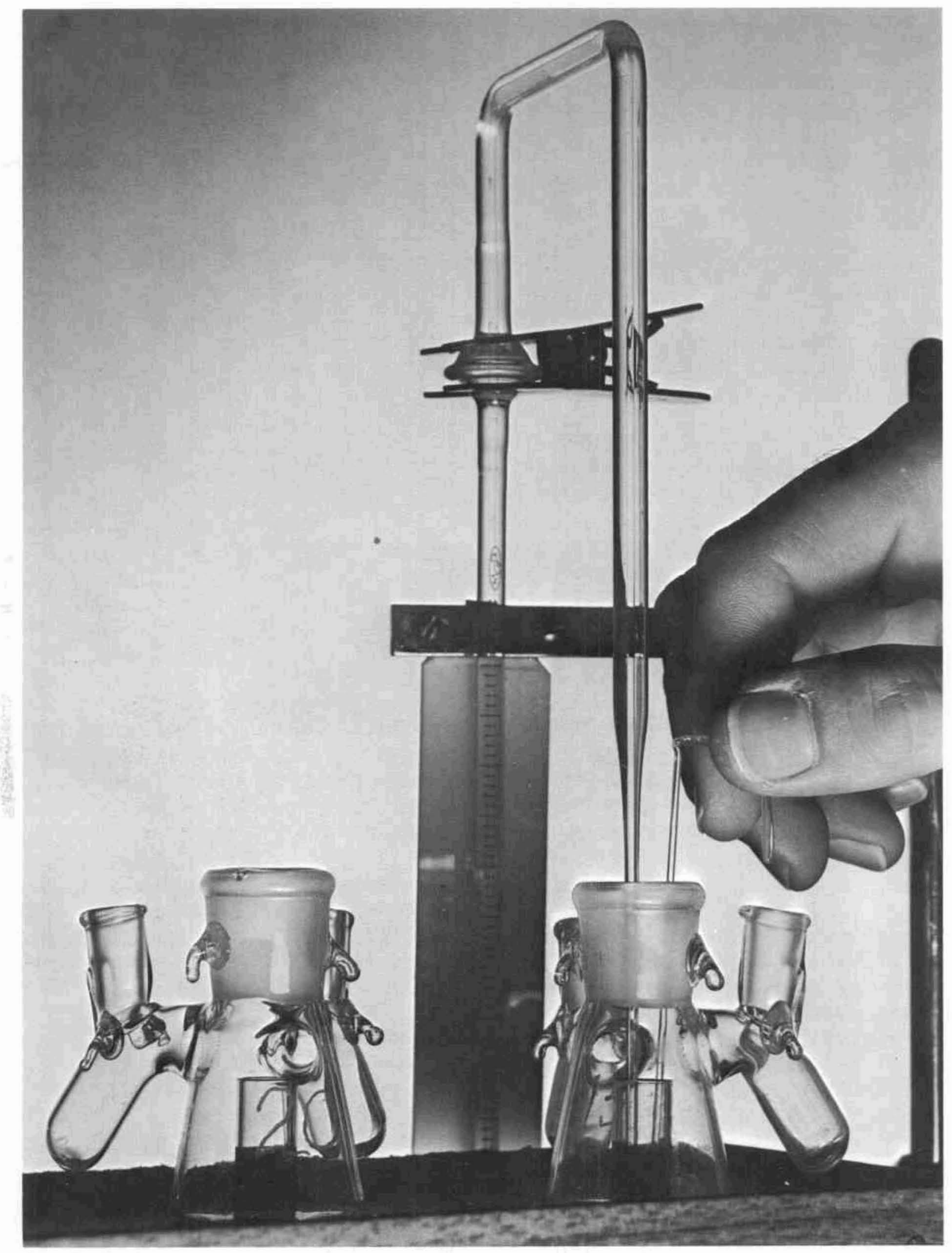

Figure 4. Microtechnique involved in studying monoamine oxidase with Warburg flasks. 
ethoxy ethanol. In view of the fact that monoamine oxidase activity is markedly increased by increasing $\mathrm{O}_{2}$ concentrations (which also affect the radiosensitivity of the system), a procedure for measurement of the enzyme's activity in a controlled atmosphere was developed. The new procedure allows the concomitant measurement of both oxidation and deamination rates in the Warburg flask. Measurements of both these rates with tyramine as substrate disclosed that the ratio $\mathrm{O}_{2} / \mathrm{NH}_{3}$ varied roughly from $1: 1$ to $1.5: 1$. Addition of catalase does not affect this ratio. Addition of several other substrates markedly increases the net oxidation in the presence of tyramine without discernible effect on the rate of deamination. This interesting phenomenon is being studied further in order to ascertain whether amines are not actually stimulating the in vitro metabolism of tissues. In any event, it is apparent that $\mathrm{O}_{2}$ uptake measurements are not always specific indicators of oxidative deaminations.

\section{ADMINISTRATIVE REPORT}

There have been no major administrative changes during the year. Total patient days in the hospital were 7028 compared with 6712 the previous year, and admissions were 95 compared with 109 . Since only 99 patient days were for nonresearch admissions, the data indicate longer stays with a slightly decreased turnover; this is compatible with the detailed type of clinical investigation being carried out. An interesting development has been the gradually widening geographical origin of the patients: 39 of the 95 patients admitted during the year came from outside New York State and from as far away as Massachusetts, Michigan, Missouri, and Mississippi. Grand rounds are held each Friday afternoon on one or more of the hospital wards, and have provided considerable stimulation to clinicians and nonclinicians alike, which is in no small part due to superior contributions by the nonclinical scientists.

The occupational therapy program continued to play a vital role in the lives of patients confined to the hospital for long periods of time. The staff in the diet kitchen earned the patients' praise for their diligent capacity to provide tasty meals despite a variety of singular limitations imposed by the investigators. The service clinical laboratories continued to function at the high level of accuracy and efficiency which has been their wont

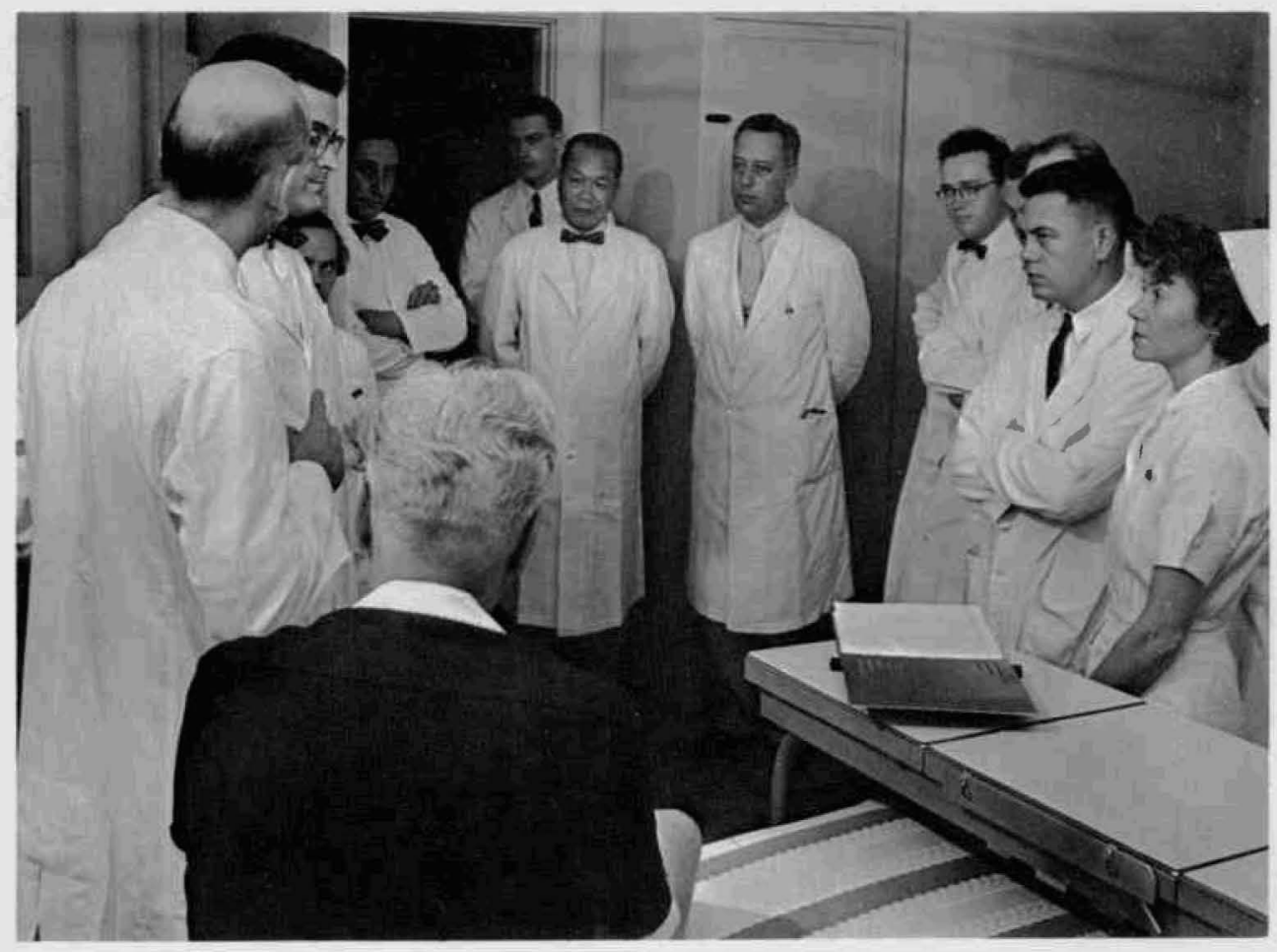

Figure 5. Friday afternoon "grand rounds" in one of the hospital wards by members of the staff. 
In the Division of Industrial Medicine, total clinic visits numbered 8850 , a considerable increase over the 7392 recorded the preceding year, and indeed the largest attendance at the clinic since the Laboratory was established. For eight months of the year this heavy work load was handled by only one full-time physician, but another full-time physician will be added to the division early in July. The number of $\mathrm{x}$-rays taken during this year remained virtually unchanged over the preceding one: 1947 vs 1973.

J. T. Godwin of the Division of Pathology developed an exhibit, "Aspiration Biopsy," which was awarded first prize among the exhibits presented at the annual meeting of the American Society of Clinical Pathologists in Chicago in October. He and E. E. Stickley of the medical physics group, Division of Physiology, prepared an exhibit entitled, "Thermal Neutron Therapy in Glioblastoma," which was awarded first prize at the annual meeting of the Radiological Society of North America in Chicago in December.

L. I. Gidez of the Division of Biochemistry shared with M. L. Karnovsky of Harvard Medical School the second prize in the annual awards of the Glycerine Producers Association for devising a chemical synthesis for incorporating radioactive carbon into glycerol and glyceride. 


\section{TECHNICAL OPERATIONS AND SERVICES}

In addition to supporting research activities, many technical service units in the Laboratory are responsible for certain applied or even basic research projects. In many cases these projects grow out of service functions as the need develops, either in the Laboratory or in the over-all Atomic Energy Commission program. A basic research project of such nature has been the primary objective of the Meteorology group in the past year. The special climatological reports and forecasts necessary for reactor operations have been continued, but the main effort was directed toward a more complete understanding of low level atmospheric dispersion. Progress in these important studies has been slow, in spite of the intensive efforts in the United States and other countries. The Brookhaven group is attacking the problem with a fresh theoretical approach, to promote a better understanding of the physical processes involved.

The Health Physics Division carried out research investigations on several types of radiation dosimetry. In particular, it concentrated considerable effort on fast neutron dosimetry.

A Remington Rand digital punch card computer was installed at the Laboratory as a joint scientificfiscal computing project. In addition to scientific computation, this machine handled the routine Laboratory fiscal operations such as payroll. For solving lengthy and complex computation problems, the Univac computer, operated by New York University, became available in the past year.

\section{REACTOR OPERATIONS}

The reactor operated at an average of 22.87 megawatts per hour of operation to accumulate a total of 7,244 magawatt days, as against 7,501 megawatt days during the preceding year. During the first half of this period, electrical power conservation measures dictated three-fan operation with resultant lower operating power levels. The average maximum power level was 23.57 megawatts; 28 megawatts was the highest power level reached during the year.

Downtime averaged 16.89 percent, of which 10.75 percent was for operational requirements and the balance of 6.14 percent for research demands. This downtime included several extended shutdowns: one for a special classified experiment with the reactor which has been reported in BNL-275, and another maintenance shutdown of two weeks' duration.

In December, the $1500-h p$, No. 5 primary fan motor burned out and was sent back to its manufacturer to be rebuilt. In February, a maintenance check revealed that the No. 4 motor was on the verge of complete failure and it has also been rebuilt. Resistance thermometers were included in the windings of these rebuilt motors and, at the present time, an additional modification is being studied to prevent accidental overloading. Recent maintenance checks of the fan motors showed them to be in good condition, requiring only routine shimming and alignment.

As a consequence of discharging and shipping irradiated fuel elements to Oak Ridge on a routine replacement schedule, modifications to the canal facility have been under consideration. The American Machine and Foundry Company has offered several studied suggestions in this connection.

Improvements of this facility have already been effected by installation of: (1) a sump pump to facilitate drainage from both spills and washdowns, and, (2) a new filter system employing diatomaceous earths as the medium which eliminates maintenance costs, time, and disposal procedures required by solid radioactive wastes.

Operations have also been simplified by the installation of a decontamination facility adjacent to the reactor, by the addition of a borescope, overhaul and modification to a periscope, and modifications to the log amplifier system.

A temperature resistance test of the exit air filters was performed to the complete satisfaction of members of the Commission's Advisory Committee on Reactor Safeguards, who were observing operations at this site.

A study has been initiated to consider the substitution in the Brookhaven reactor of enriched fuel, and a preliminary proposal to the Commission has been prepared. 


\section{ISOTOPE DISTRIBUTION AND SPECIAL MATERIALS}

As in the preceding year, the activities of the Isotopes and Special Materials group centered on the processing and shipment of radioisotopes and on service irradiations to external users. The volume of shipments increased by 12 percent. The number of different customers that have been served increased from 82 to 123 , of which 91 received shipments this year. Also of interest is the fact that shipments have been made to 23 different states.

In addition to this outgoing service, 130 orders for isotopes and 32 orders for special materials were placed with the Commission and other suppliers. Against these, approximately 245 shipments were received within the Laboratory.

The S/F Accountability Program, as carried out by this group, involved the monthly preparation and submission to the Commission of ten categories of

Tabıe 1

\begin{tabular}{|c|c|c|c|c|c|c|c|c|}
\hline \multicolumn{9}{|c|}{ Summary of reactor services } \\
\hline \multirow[b]{2}{*}{ External Users } & \multicolumn{2}{|c|}{$1954-1953$} & \multicolumn{2}{|c|}{$1953-1952$} & \multicolumn{2}{|c|}{$1952-1951$} & \multicolumn{2}{|c|}{$1951-1950$} \\
\hline & $\begin{array}{l}\text { No.of } \\
\text { Irradiations }\end{array}$ & $\begin{array}{c}\$ \\
\text { Billed }\end{array}$ & $\begin{array}{c}\text { No.of } \\
\text { Irradiations }\end{array}$ & $\begin{array}{c}\$ \\
\text { Billed }\end{array}$ & $\begin{array}{c}\text { No.of } \\
\text { Irradiations }\end{array}$ & $\begin{array}{c}\$ \\
\text { Billed }\end{array}$ & $\begin{array}{l}\text { No.of } \\
\text { Irradiations }\end{array}$ & $\begin{array}{c}\$ \\
\text { Billed }\end{array}$ \\
\hline Target Conveyor & 799 & 40,899 & 737 & 40,590 & 464 & 18,663 & 124 & 3,974 . \\
\hline Pneumatic Tubes & 41 & 1,316 & 68 & 1,495 & 575 & 2,931 & 60 & 260. \\
\hline Air-cooled Holes & 28 & 6,492 & 12 & 4,175 & & & & \\
\hline Water-cooled Holes & 16 & 1,726 & 24 & 825 & & & & \\
\hline Thermal Column & 6 & 330 & 18 & 912. & & & & \\
\hline Experimental Holes & 42 & 7,633 & 24 & 6,533 & 8 & 5,971 & & \\
\hline Special & 36 & 50. & 12 & 190. & & & & \\
\hline Cyclotron & 4 & 275. & & & & & & \\
\hline Sub-totals & 972 & 58,721 & 895 & $54,720$. & 1,047 & 27,565 & 184 & 4,234 \\
\hline $\begin{array}{l}\text { Multicurie Co Sources } \\
\text { Rentals }\end{array}$ & 3 & $\begin{array}{l}15,185 \\
68,943\end{array}$ & 5 & $\begin{array}{l}52,382 \\
61,493\end{array}$ & 2 & $\begin{array}{l}11,750 \\
24,563\end{array}$ & & \\
\hline Totals & 975 & 142,849 & 900 & 168,595 & 1,049 & 63,878 & 184 & 4,234 \\
\hline \multicolumn{9}{|l|}{ Internal Users } \\
\hline Target Conveyor & 566 & 23,826 & 329 & 38,982 & 288 & 18,443 & 138 & 8,433 . \\
\hline Pneumatic Tubes & 911 & 4,555 & 888 & 4,440 & 819 & 4,095 & 211 & 1,055 \\
\hline Totals & 1,477 & 28,381 & 1,217 & 43,422 & 1,107 & 22,538 & 349 & 9,488 \\
\hline
\end{tabular}

Table 2

Four-year summary of shipments by states, 1950-1954

\begin{tabular}{lrlr}
\hline New York & 1,118 & Connecticut & 11 \\
Massachusetts & 588 & Indiana & 9 \\
Tennessee & 143 & Wisconsin & 8 \\
New Jersey & 125 & Oklahoma & 8 \\
Minnesota & 94 & Michigan & 8 \\
(Canada) & $(91)$ & Washington, D. C. & 7 \\
California & 74 & Louisiana & 4 \\
Pennsylvania & 58 & Missouri & 4 \\
Texas & 30 & Nebraska & 3 \\
Ohio & 23 & Iowa & 3 \\
Illinois & 23 & Rhode Island & 1 \\
Maryland & 15 & Alabama & 1 \\
& & & \\
\hline \hline
\end{tabular}

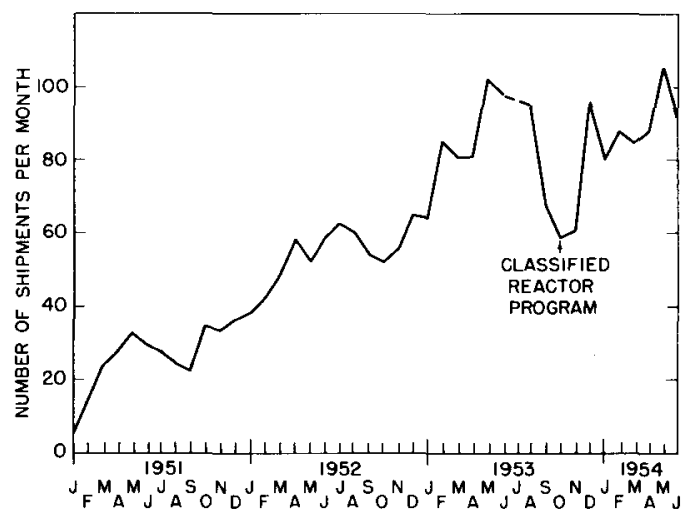

Figure 1. 
inventories. A revision of the manual, "Procedures for Handling S/F Materials," was issued in July 1953. During that same period, an intensive survey of procedures for accountability of S/F materials, together with a physical check of inventories, was made by Commission representatives. During the year, periodic shipments of irradiated fuel were made to Oak Ridge and arrangements have been initiated for returning to the Commission a large quantity of unused S/F material.

The 1954 total number of shipments was 970; of these, 354 went to hospitals, 304 to universities and 312 to industrial organizations and government agencies.

\section{RADIATION APPLICATION ENGINEERING}

During the past fiscal year, 16 gamma-ray sources were fabricated, 15 of the standard type, and one custom-made to the requirements of Tracerlab, Inc.

\begin{tabular}{|c|c|c|c|}
\hline \multicolumn{4}{|l|}{ Table 3} \\
\hline National Canners & 38.75 & \multicolumn{2}{|c|}{ source days } \\
\hline Standard Oil & 297.00 & " & "' \\
\hline Evans Signal Laboratory & 0.25 & $"$ & $"$ \\
\hline Dow Chemical & 7.0 & $"$ & $"$ \\
\hline General Foods & 2.0 & $"$ & $"$ \\
\hline Ethicon Suture & 0.166 & $"$ & $"$ \\
\hline Yale University & 30.0 & " & $"$ \\
\hline Gilfillan & 2.0 & $"$ & $"$ \\
\hline Naval Research Lab. (Brooklyn) & 15.0 & $"$ & $"$ \\
\hline Naval Research Lab. (Washington) & 61.5 & $"$ & $"$ \\
\hline Congoleum Nairn & 23.0 & $"$ & $"$ \\
\hline Bakelite Corp. & 12.0 & $"$ & $"$ \\
\hline US Rubber Co. & 1.0 & $"$ & $"$ \\
\hline Radio Corp. of America & 2.0 & 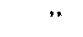 & " \\
\hline Army Quartermaster Corps & 3.0 & $"$ & $"$ \\
\hline \multirow[t]{2}{*}{ Wright Aeronautical } & 10.0 & $"$ & " \\
\hline & $\begin{array}{l}504.166 \\
\text { ays) }\end{array}$ & $"$ & " \\
\hline
\end{tabular}

\begin{tabular}{|c|c|c|}
\hline \multicolumn{3}{|l|}{ Table 4} \\
\hline \multirow{8}{*}{$\begin{array}{l}\text { Fission Products Utilization Project } \\
\text { Nuclear Engineering } \\
\text { Chemistry Department } \\
\text { Biology Department } \\
\text { Physics Department } \\
\text { Health Physics Division } \\
\text { Pile Operations } \\
\text { Picatinny Arsenal }\end{array}$} & 1286 & source days \\
\hline & 328 & $" \quad "$ \\
\hline & $72.33^{\mathrm{I}} / 3$ & " \\
\hline & $2.16^{2} / 3$ & 3 \\
\hline & 163 & $"$ \\
\hline & 67 & $"$ \\
\hline & 3 & \\
\hline & 9 & $"$ \\
\hline \multicolumn{3}{|c|}{$\begin{array}{l}1930.50 \\
\text { (Fiscal '53-1583 source days) }\end{array}$} \\
\hline
\end{tabular}

\begin{tabular}{ccccc}
\hline Table 5 & & \\
\hline $\begin{array}{c}\text { Source } \\
\text { No. }\end{array}$ & Description & Buyer & $\begin{array}{c}\text { Curie } \\
\text { value }\end{array}$ & $\begin{array}{c}\text { r/hr at } \\
\text { geometric } \\
\text { center }\end{array}$ \\
\hline Cobalt-17 & $\begin{array}{c}\text { Standard, } \\
1 / 8 \text { in. wall }\end{array}$ & B. F. Goodrich & 530 & 203,000 \\
Cobalt-24 & $\begin{array}{c}\text { Standard, } \\
1 / 8 \text { in. wall }\end{array}$ & Wright Field & 1357 & 520,000 \\
Cobalt & $\begin{array}{c}\text { Custom-made, } \\
1 / 16 \text { in. wall }\end{array}$ & Tracerlab & 125 & 135,000 \\
\hline \multicolumn{4}{c}{$\begin{array}{l}\text { Sources ordered - still in reactor: } \\
\end{array}$} & $\begin{array}{l}\text { Cobalt } 25 \text { for Standard Oil } \\
\text { Cobalt } 23 \text { for Dow Chemical } \\
\text { Co 1-2-3-4 for B. F. Goodrich } \\
\text { (Flat sources) }\end{array}$ \\
\hline \hline & & \\
\hline
\end{tabular}

Twenty-four cobalt sources and 3 tantalum sources were put into the reactor, while 16 cobalt sources and 2 tantalum sources were discharged.

At this time, 26 cobalt sources and 2 tantalum sources are in the reactor, with 7 more cobalt sources ready to go in. Two-hundred flat cobalt sources are on order.

The following sources are in service: 3 tantalum sources (one in the water pit), 7 cobalt and an additional 15 cobalt in the water pit.

Table 3 is a list of the off-site users of the gamma irradiation facility and the time used in source days for the 1954 fiscal period.

Next is a list of the BNL users and the time used in source days for 1954 fiscal period.

With an average of 10 sources in use during the last fiscal period, the total source days available were 3650 . Of these, 2434 source days were used, representing 67 percent of the available time. Discounting weekends and holidays, the source time available would be 2510 days, and the utilization percent would be 97 .

The cobalt sources sold and delivered during the ' 54 fiscal period are given in Table 5 .

\section{HOT LABORATORY OPERATIONS}

\section{RADIOGRAPHY}

During the past year equipment was built and several sources were procured with which to radio- 
graph a wide variety of objects. Standard techniques were learned and new ones have been and are still being developed, and, as a result, radiography is now done as a routine service.

During the last six months of the year, a total of 508 radiographs were made, 424 of these were for other groups at the Laboratory and 84 were used in developing and improving techniques.

As an example of these new developments, an iridium-192 source was made which is so small in physical size that it can be inserted inside a tube 1 in. in diameter in order to radiograph that tube. The film is wrapped around the tube and the resulting exposure is much clearer and easier to interpret than one obtained by the standard technique of placing both the source and the film external to the object being radiographed. A self-centering source holder was developed for this purpose, and a still smaller source is being made for use on smaller diameter tubes.

Another example of this developmental work is the use of radiography to give information other than that on defects in vessel walls and welds. In one case, radiography showed the existence and location of material which had precipitated out of a circulating loop that contained a molten metal; this qualitative information was of interest in the process going on in the system. An attempt will also be made to use radiography to indicate not only the liquid level in a metal-walled dissolving vessel, but also the location and number of metal slugs being dissolved in the vessel. In a down-to-earth practical application, radiography was recently used to locate the reinforcing steel in a concrete floor preparatory to drilling into the floor.

\section{Equipment Development}

Although equipment development has been hampered by transfer of manpower to other areas, some accomplishments can be noted. A second manipulator (ModeI II) was designed and constructed and placed in satisfactory operation in one of the semi-hot laboratories. Patterned after the Model I manipulator in use in the sample preparation room, the Model II has many significant improvements in the ease and smoothness of operation.

A small pneumatically operated bellows-type valve has been designed and built and found to operate quite satisfactorily. It is patterned somewhat after a Hanford valve with some improvements.

\section{Special Sources}

Considerable work has been done in making pure $\beta^{-}$-emitting sources for local intense irradiation for use by members of both Biology and Medical Departments. One such source of thallium metal (4-yr $\mathrm{Tl}^{204}$ ) is mounted in a retractable holder in such a way that it can be used conveniently to irradiate the eye of a rat or a mouse. Another, consisting of three small thallium wires encased in very fine polyethylene tubing, again held in a retractable mount, is arranged so that the source can be lowered into a slowly rotating test tube containing the solution to be irradiated; it is small enough so that the entire unit can be placed in an incubator. Some consideration has also been given to making a needle emitting only alphas.

\section{Liquid Waste Operation}

The Waste Concentration Plant has been in continuous day-shift operation since August, 1953. (Some delay was encountered in July when the large vapor compressor was found to be defective and had to be returned to the manufacturer for repair.) Operation since August has been quite satisfactory and was interrupted only once when the glass wool spray catcher was replaced by copper mesh having a longer life. A roof was added over the loadingstorage platform to permit easier operation during inclement weather, and a hopper and conveyor system were installed to simplify the handling of the Portland cement used to solidify the waste residue.

In cooperation with the Ultimate Waste Disposal group, some experiments are in progress on absorbing radioactive material out of solutions on to a clay column. Two purposes are behind this work: (1) used in conjunction with the present evaporative waste concentration process, a preadsorption on clay will probably render more economical the processing of dilute large volume wastes such as canal water, (2) with highly radioactive wastes, such a clay column probably will turn out to be a useful source of $\beta^{-}$and $\gamma$ radiation for other experimental purposes.

The figures below indicate the magnitude of the liquid waste operations for the past year. It will be noted that although the volume of "D" waste received during the year was more than quadrupled over what it was the previous year, nevertheless, the concentration plant was able to handle this and more, resulting in a net decrease of 11,364 gallons of " $D$ " waste in storage. 
Table 6

"F"' Waste discharged to the sewer

\begin{tabular}{cccc} 
Period & Volume, gal & $\begin{array}{c}\text { Average activity } \\
\text { level } \mathrm{c} / \mathrm{ml}\end{array}$ & $\begin{array}{c}\text { Total } \\
\text { activity mc }\end{array}$ \\
\hline Fiscal 1953 & $3,124,822$ & $5.64 \times 10^{-12}$ & 66.16 \\
Fiscal 1954 & $2,078,471$ & $2.05 \times 10^{-12}$ & 163.89 \\
\hline
\end{tabular}

Waste Concentration

\begin{tabular}{|c|c|c|c|c|}
\hline \multicolumn{3}{|c|}{$\begin{array}{l}\text { Feed, gal } \\
\text { Average volume reduction } \\
\text { Drummed concentrate, gal } \\
\text { Number of drums of concentrate }\end{array}$} & \multicolumn{2}{|c|}{$\begin{array}{r}435,953 \\
90: 1 \\
4,929 \\
198\end{array}$} \\
\hline \multicolumn{5}{|c|}{ Waste Inventory } \\
\hline & Fiscal & 953 & Fiscal & 1954 \\
\hline & $\begin{array}{l}\text { "D" } \\
\text { Waste }\end{array}$ & $\begin{array}{l}\text { "A" } \\
\text { Waste }\end{array}$ & $\begin{array}{l}\text { "D" } \\
\text { Waste }\end{array}$ & $\begin{array}{l}\text { "A" } \\
\text { Waste }\end{array}$ \\
\hline $\begin{array}{l}\text { Volume on hand at } \\
\text { beginning of fiscal } \\
\text { year, gal }\end{array}$ & 90,102 & 347 & 168,704 & 1,590 \\
\hline $\begin{array}{l}\text { Volume received } \\
\text { during year, gal }\end{array}$ & 100,581 & 1,243 & 424,589 & 168 \\
\hline $\begin{array}{l}\text { Volume to } \\
\text { evaporator, gal }\end{array}$ & 21,979 & 0 & 435,953 & 0 \\
\hline $\begin{array}{l}\text { Volume on hand at } \\
\text { end of fiscal } \\
\text { year, gal }\end{array}$ & 168,704 & 1,590 & 157,340 & 1,758 \\
\hline
\end{tabular}

\section{HEALTH PHYSICS}

The work of the Health Physics Division of the Instrumentation and Health Physics Department has expanded gradually during the year, both in regard to radiation safety problems and in regard to research and development activities. There has been the usual emphasis on training activities for the Division's people, visitors and the AEC Fellows in Radiological Physics.

A revised edition of the Brookhaven Safety Manual was prepared and approved by the Radiation Safety Committee. Averaging of personnel exposures over 13 weeks was allowed, the limit for off-site radiation levels was set at $30 \mathrm{mr} / \mathrm{wk}$ and Bureau of Standards Handbook 52 was adopted as the standard for maximum permissible concentrations of activity in air and water. The Counting Room Manual was revised to include the latest equipment modifications and will be used for training purposes.
A new chemistry laboratory was set up at the central Health Physics building to handle the increasing number of urine samples that must be analyzed for radioactive contaminants. Such samples were analyzed for all pile operations and Health Physics personnel using the calcium oxalate precipitation method; no significant body burdens of fission product were detected.

\section{RESEARCH AND DEVELOPMENT}

The Health Physics Division does not have a separate staff for research work but carries on considerable research and development work concurrently with its service functions. Most of this work is concerned with radiation dosimetry in one way or other. An improved facility for calibrations at high radiation intensity has been set up in an empty building. A 50-curie $\mathrm{Co}^{\mathrm{B0}}$ gamma-ray source and a 1-curie radium-beryllium fast neutron source are provided.

There have been a number of investigations connected with fast neutron dosimetry. A more sensitive portable survey meter of the $\mathrm{ZnS}$-lucite variety was constructed in which a 5-in. diameter photomultiplier tube was used. Values of neutron flux above $20 \mathrm{Mev}$ at the Cosmotron were obtained by measuring the activation of a large anthracene crystal with a photomultiplier tube. Fluxes as small as $5 \mathrm{n} / \mathrm{cm}^{2} / \mathrm{sec}$ can be detected in this manner. An ionization chamber made of tissue-equivalent plastic was constructed and incorporated in a portable survey meter for Cosmotron use. A set of lithium metal shields has been fabricated and is being used in conjunction with thimble chambers and film dosimeters to determine the gamma-ray dosage rate in the irradiation chamber of a thermal column on top of the nuclear reactor.

Other projects included a study of the absolute yield of the ferrous sulfate dosimeter (in cooperation with the Electronics and Instrumentation Division and the Chemistry Department), identification of $\mathrm{Cs}^{137}$ as the chief radioactive constituent of pile canal water, and measurements of dosage rate at the Biology field, inside several kilocurie sources and at several other irradiation facilities.

\section{WASTE DISPOSAL}

The quantity of radioactive solid waste accumulated at the waste control area has continued to increase and currently amounts to 60 drums a month, 
or about $31 / 2$ times as much as a year ago. A permanently installed splash-proof concrete mixer has been provided and has greatly improved efficiency. A technique has been developed for pouring concrete liners for drums. Very radioactive waste items from the pile are put directly into these drums which provide needed shielding en route to the waste area.

During the week of April 26th, 476 barrels of radioactive waste materials were dumped at sea beyond the edge of the continental shelf by the Navy. The shipment weighed 206 tons and contained an estimated 233 curies of activity. The operation was completed without a single exposure of more than the weekly limit even though many of the barrels read well over $1 \mathrm{r} / \mathrm{hr}$ at the surface. Survey coverage was provided for all parts of the operation, including unloading at sea. Personnel monitoring equipment was provided for the 92 Navy personnel involved in the operation.

Enough experience has been accumulated so that some idea of the cost of sea disposal of solid wastes at Brookhaven can be obtained. Dumping at sea is currently done without charge by the Navy but would be done by a commercial bidder for about $\$ 10.00$ per drum. Packaging the solid wastes in concretelined drums costs $\$ 3.35$ per drum, and getting them from the Laboratory on board ship costs $\$ 2.60$ per drum. There is additional expense in collecting and processing the wastes and in providing and maintaining the necessary equipment and facilities.

The emission constant and shielding effect of concrete for the fission products that account for most of the activity packaged for sea burial were determined experimentally so that a satisfactory value of curie content of each drum can be derived from the reading of a survey meter at a standard distance from the surface of the drum. Most of the activity disposed of is $\mathrm{Cs}^{137}$, a fission product which appears to be readily dissolved by the water of the pile canal.

Underground storage pits and trenches provided a year ago have been useful for the very active apparatus that is occasionally removed from the pile. Safe techniques have been worked out for transferring equipment, with levels up to $100 \mathrm{r} / \mathrm{hr}$ at $10 \mathrm{ft}$, from experimental holes in the reactor to the storage trenches at the waste area.

The volume of liquid waste discharged increased somewhat during the past year, but the concentration of activity discharged to the filter beds showed a decrease. The discharged activity continues to average about 10 percent of the amount permitted by the currently prescribed limit.

\section{SURVEY ACTIVITIES}

There has been a large volume of survey work at the Cosmotron where the radiation levels have varied widely, depending on the type of operation, the shielding arrangement and the performance of the machine. The radiation levels for full intensity operation in June 1954 are shown in Figure 2. The values are given in $\mathrm{mrem} / \mathrm{hr}$ based on a biological effectiveness ratio of 10 and measured with a tissue-equivalent chamber. Operation at full intensity occurs only part of the time, so that average levels are much lower and there are only a few areas where it is necessary to limit the access of personnel. Levels even higher than those shown in Figure 2 occur under special conditions, for instance, with certain targets in the south or west straight sections, or when the proton beam is caused to expand in radius at the end of an acceleration cycle. This latter condition approximately doubles most of the levels but increases some of those in the motor generator room by a factor of 10. Special arrangements are, of course, necessary for this type of operation.

A monitor operating continuously in the Cosmotron control room averaged 213, 155, 148 and 216 mrem/wk for the four quarters of the year just ended. The lower levels during the fall and winter were caused by the addition of extra shielding over the east straight section and the increase in levels this spring resulted from improvements in the operating efficiency of the machine.

The Cosmotron presents a difficult problem in exposure control because of the large areas where there are substantial radiation levels and where scientific work must be carried on. Improvements in the shielding are contemplated, but further improvement in the efficiency of the machine is also to be expected.

The volume of Health Physics survey work at the reactor continues to be large and varied, particularly during shutdowns when fuel elements are loaded or unloaded. A room next to the Health Physics office equipped for checking personnel for contamination and for simple decontamination jobs has been very useful. One of the area monitoring dust monitors has been adapted for use with a logarithmic ratemeter to monitor particulate activity in 


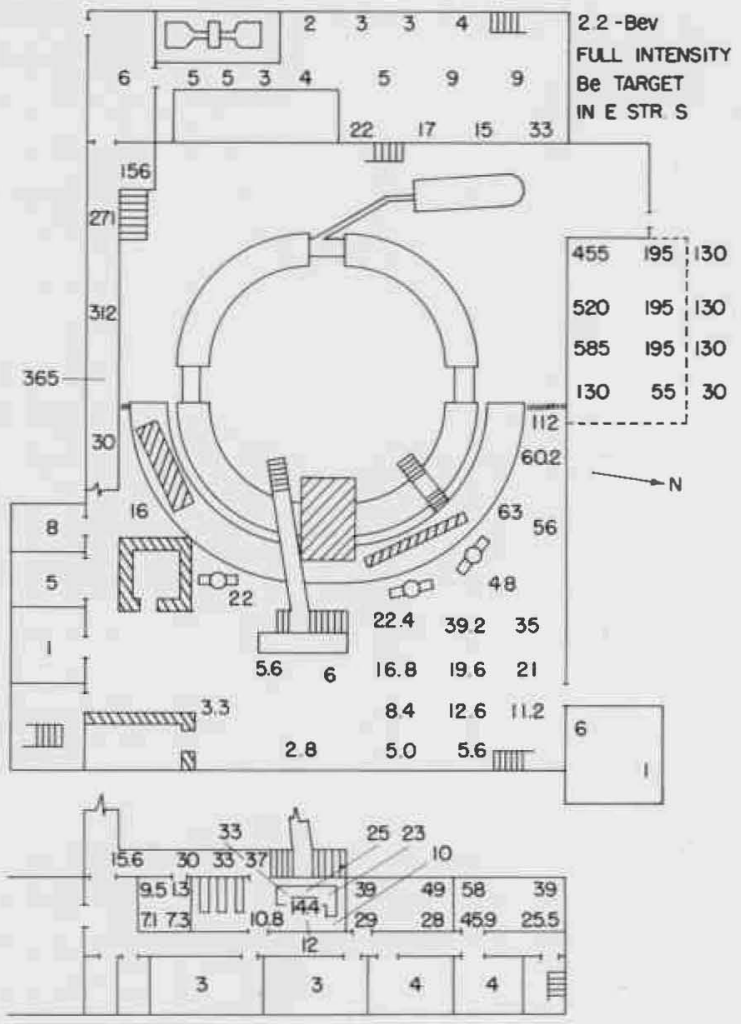

Figure 2. Radiation levels at the Cosmotron in $\mathrm{mrem} / \mathrm{hr}(\mathrm{rbe}=10)$.

the pile cooling air. Exposure and contamination control in the pile canal area has been especially troublesome but the necessary work has been done safely as a result of frequent surveys and continual improvement of techniques.

Four of the off-site area monitoring stations have been closed now that levels from the pile are well established and have been shown to be well below permissible values. Two off-site stations have been retained for reference and calibration purposes. Considerable data on fallout resulting from atomic weapons tests are obtained from on-site monitoring stations and from daily analyses of rainwater and settled dust. The on-site stations continue to be operated for this purpose and to detect any additional disturbances to background that might result from Brookhaven Laboratory operations.

\section{PERSONNEL MONITORING}

The scale of operations of the personnel monitoring group as shown in Table 7 remained about the same as last year except for an increase in neutron monitoring caused by the growing program at the
Table 7

Use of personnel monitoring equipment

\begin{tabular}{lrr}
\hline & $1952-53$ & $1953-54$ \\
\hline Pairs of pocket chambers & 1,313 & 1,330 \\
Regular film badges & 903 & 844 \\
Visitors' film badges-On-site & 167 & 230 \\
Visitors' film badges-Off-site & 135 & 124 \\
Total film badges & 1,205 & 1,198 \\
Neutron films used & 369 & 214 \\
Neutron films read & 223 & 115 \\
\hline
\end{tabular}

Figures have been averaged on a per week basis.

Cosmotron. The off-site neutron monitoring service grew gradually, the average number of badges serviced being 220 per week in addition to those shown in Table 7. This service has now leveled off at about 300 badges per week which are furnished for 21 different laboratories.

Radiation exposures of employees continued low for the most part and in no case was the currently accepted limit of $300 \mathrm{mrem} / \mathrm{wk}$, averaged over a 13-wk period, exceeded. All cases of more than 300 mrem exposure in any week were investigated as a precautionary measure; there was an average of 5 such investigations per week.

An analysis of the personnel monitoring records of 146 individuals who worked regularly at the pile from July to December 1953 showed an average exposure of $27 \mathrm{mrem} / \mathrm{wk}$. Forty-three individuals averaged 10 percent or more of the $300 \mathrm{mrem} / \mathrm{wk}$ that is permissible, the highest being 54 percent. The increase in exposures, as compared with last year, is substantial, reflecting the increased volume of work in high radiation fields necessary to operate and maintain the pile.

A careful check has been made of personnel exposures at the Cosmotron by means of NTA films worn by everyone working in that area. Conditions have varied during the year but the highest average exposure was 40 percent of maximum permissible an'd 20 individuals averaged 20 percent or more of the limit.

\section{INSTRUMENTATION}

The research and development activities of the Electronics and Instrumentation Division have been 
of synoptic and tower data to both IBM and Remington Rand machine punch cards. These data include hourly values of all temperature and wind records obtained from the tower installation as well as complete synoptic observations and parameters calculated from standard weather charts. In June, 1954, a new Air Force project was initiated. This will include a complete machine processing of the cards prepared in the first project to provide a clear picture of the relationship between the synoptic meteorological variables and the micrometeorological data. The purpose of this new work will be twofold: (1) to provide forecasters having available only the usual meteorological information with a means of predicting detailed wind and temperature conditions in the lower levels of the atmosphere, and (2) to provide an analysis of the variations found on a group of selected days having extremely uniform general weather conditions.

\section{GROUND WATER SURVEY - US GEOLOGICAL SURVEY}

During the period from July, 1953 to July, 1954, M. A. Warren and W. deLaguna, of the Ground Water Branch of the US Geological Survey, completed a draft of the report covering the previous six years of work on the question of possible water resources contamination by Laboratory operations. Although considerable editing and review by the Office of the Director of the Geological Survey still remain, the bulk of the work has been finished.

The general conclusions of the report are as follows:- Liquids, containing radioactive materials, accidentally released to the ground might be rendered harmless by any one of three processes: dilution, adsorption, and radioactive decay.

Dilution is not a factor on which much reliance can be placed, particularly in the case of a large spill. Adsorption is a process which is very difficult to evaluate; its effectiveness depends upon the chemical composition of the liquid involved. It is possible, however, that only a few ions, like ruthenium and iodine, are able to move with any freedom through the soil. The effects of decay can be more exactly determined.

The ground water in the saturated glacial sand under the Laboratory is moving at a rate not in excess of $1 \mathrm{ft}$ per day. Contamination in the water can advance, then, at most only 300 to $400 \mathrm{ft}$ in one year, and well over 10 years are required for such contamination to travel one mile. Because of this slow movement, only isotopes with relatively long half-lives can be regarded as potentially dangerouseven with respect to the Laboratory's own water supply. As these long-life isotopes must also be able to resist adsorption by the soil, the possible danger to the Laboratory or to the surrounding community from any conceivable accident is quite negligible.

Even if unforeseen circumstances should result in the release of large quantities of long-life radioisotopes which threaten to contaminate the water supplies of this area, a number of apparently practical remedial measures have been developed which could be employed to alleviate the situation.

\section{MECHANICAL ENGINEERING AND DESIGN}

The Mechanical Engineering Division employs its staff in the preparation of estimates of contemplated projects, the design of machines and equipment, the requisitioning of mechanical components, and the test and supervision of newly installed devices. The engineers and designers form an integral part of the departments in which work is initiated, and the equipments that have been built during the fiscal year are described in the departmental reports.

Designs of the mechanical components of an electron analogue for the proposed 25-Bev alternating gradient synchrotron have been made, and the machine is now being assembled. At the Cosmotron, styrofoam vessels with partial liquid nitrogen shielding have been used to contain liquid hydrogen in meson scattering experiments, and designs of a large quadrupole focusing magnet have been completed. The Nuclear Engineering group has built apparatus to secure engineering data and has made a preliminary design study of a liquid metal fuel reactor. Among the designs of the central group is a roof facility at the reactor, which permits the selective exposure of patients who are undergoing boron capture therapy to neutrons from the pile.

The design of the electron analogue, coupled with preliminary studies of the alternating gradient synchrotron, has forced the Division to increase its size. The Division employed 44 individuals at the end of the fiscal year, including 11 scientific, 30 tech- 
nical and 3 secretarial staff members, who comprise groups of the following size: Accelerator Development Department, 16; Central Design, 9; Cosmotron, 8; Nuclear Engineering, 8; Cyclotron and Van de Graaff, 2; Physics, 1.

\section{MACHINE SHOPS}

The major effort of the Central Shops organization has continued to be directed toward the fabrication of parts and equipment in the support of research. At the end of the report year there were 13 jobs under construction which required more than 500 man-hours each before being completed. Approximately 2500 man-hours had already gone into the preliminary design and development work for the new 25-Bev accelerator.

Considerable turnover was experienced in Central Shops personnel. This resulted primarily from the Laboratory's practice of attempting to replace Staff Shop machinists by machinists from the Central Shops group. Nine such transfers took place while only one termination occured. Replacements were not made on a man-for-man basis, and the employment level at year end stood at 69 as compared with 75 for the same date a year ago.

The relative demand for welding and sheet metal work increased throughout the year. This was in large measure the result of chemical engineering programs in the Nuclear Engineering Department which have required services of two welders essentially full time. Thus, of the new employees, a higher percentage was added in these crafts.

Major jobs in Shops completed or in progress are as follows:

Armor plate shield and rotor for fast chopper

Lattice experiment

Volatility project

Crystal spectrometers

Special lead containers and shipping shields

Electron spectrometer

Projectors for cloud chambers

Fuel cartridge chopper parts

Water-cooled facility (Reactor)

In-pile loop for LMFR

Vacuum dry box

Irradiation facility (for brain tumor work at reactor)

AGS test models, equipments, etc.

\section{TECHNICAL INFORMATION}

The staff of the Technical Information Division numbered 21 during this review period, the same figure as for the two previous years. However, as noted last year, there has been a continuance of the steady increase in the demands on the various services of the Division. This increase can be readily explained by the over-all expansion of the Laboratory's scientific program and the growing uses of its research facilities.

In the Research Library, a gain in activity is noted in all sections, but it is particularly apparent in the Circulation and Reference sections. In addition to the increasing use being made of the book and periodical collection by the Laboratory staff, a significant gain has occurred in loan requests from universities, industrial firms and medical centers in the northeastern area. The general science collection, started over a year ago with the support of Associated Universities, Inc., is steadily becoming more popular as the selections are increased. This collection covers mainly the history and philosophy of science. The collection of translated Russian scientific articles continues active, both in new acquisitions and in loans.

The Reference section is being called upon more frequently to prepare bibliographies and literature searches. Such requests have more than doubled during this review period.

For the first time, evening attendance figures in the reading room were kept during the summer months. The results show an additional 1500 manhours in the June-August period. For the entire year, 3000 man-hours more were recorded than in the preceding report period.

With the rapid decrease of available shelving space in the stack area, 12 three-foot units were added, and 25 filing cabinets containing unclassified reports were removed to a room formerly used for individual study. It is estimated that this change will result in stack space for two more years.

Now that the AEC's program for the reproduction of reports on microcard is well established, an evaluation of the saving of filing facilities and staff time can be made. The saving of space for 2300 reports has been accomplished to date by this means; in addition to this advantage, considerably less work is spent in locating and charging out reports to users.

In the Classified Library, the report retirement program has continued, and is now half completed. 
By this means, surplus copies of classified documents have been destroyed and substituted by microcard, with a resultant saving of critical vault space and the facilitation of inventories. Some 7000 items are now held in microcard form, out of a total of over 25,000 classified research and development reports.

A year ago, the Chief of the Declassification Branch, USAEC, spent several weeks at the Classified Library reviewing memoranda, letters, photographs and drawings that had originated here before January 1,1951, with a view to declassifying as much of this material as possible. Over 1300 items were cleared by the AEC declassification and patent procedures; the tasks of physically declassifying those copies at Brookhaven, and of notifying outside recipients of the declassification step, have just been completed.

Of the total of 304 persons authorized to have access to classified material, about 160 use the library facilities frequently, and 140 of them have material charged out to them, for storage in properly approved files. There are 34 consultants, members of visiting committees, and AUI trustees, and four Brookhaven Area AEC staff members, who hold such authorization.

The Publication group has edited and otherwise processed for publication 57 major technical documents orginating within the Laboratory, including 23 classified reports. With the Library as the coordinating agency, the Weekly Selected Reading List continues to appear on a routine basis; this useful guide to the current literature is distributed to 560 staff members and to 820 research workers at other sites.

In addition to its responsibilities in the publication of Brookhaven reports. the Division also handles all technical manuscripts originating here for publication in the scientific and professional journals. During the past year, 381 such papers (including published abstracts of speeches) have been processed, a 25 percent increase over the figure reported for last year. Since the Laboratory's research program is still expanding, it is anticipated that the volume of papers has not yet reached its plateau level.

The distribution lists for the Laboratory's unclassified reports continue to grow. All bonafide requests from within the atomic energy project and from cooperating Government agencies and universities are honored; others are referred to the official sales agency for AEC reports, the Office of Technical
Services, Department of Commerce, where all unclassified Brookhaven reports of possible interest to the public are available at a minimal cost. From the Laboratory's progress reports, reprints of the pertinent sections are sent to interested scientists at the nine Associated Universities, with a view toward fostering closer interest at the universities in the research program at Brookhaven.

\section{PHOTOGRAPHY AND DUPLICATING}

The integrated short-run copy section has been organized during the past year. This was achieved by moving the photostat machine to the Illustration building, where the Ozalid and Xerox machines are also located.

The space thus released in the photography building was used to install a process camera and otherwise relieve the crowded conditions throughout the group. The process camera has appreciably reduced the time required for making copy negatives and is permitting much greater accuracy in measuring techniques and in lens evaluation. Further equipment for photomicrography has been added.

\begin{tabular}{lrrr}
\hline & Table 8 & & \\
\hline & \multicolumn{1}{c}{ Fiscal } & \multicolumn{1}{c}{ Fiscal } & \multicolumn{1}{c}{ Fiscal } \\
& 1952 & 1953 & 1954 \\
\hline Photographic negatives & 4,344 & 4,488 & 4,662 \\
Photomicrographs & 1,471 & 1,334 & 1,435 \\
Lantern slicles & 2,983 & 3,487 & 4,489 \\
Prints & 25,393 & 29,491 & 30,302 \\
Photostats & 32,789 & 30,271 & 22,226 \\
Ozalid prints & 187,046 & 197,429 & 233,821 \\
16 mm film processed & 10.0 miles & 10.8 miles & 13 miles \\
& & & \\
\hline
\end{tabular}

The accumulation of negatives has made problems both of storage space and accessability. Currently the files are being put into smaller physical size and a contact print file on Kardex is being broken down by department and project. Though it will take considerable time to complete this, the work already done has proved most useful.

The Illustration section has prepared 2509 drawings for slides and publications and has worked on the design of a number of exhibits and displays.

The Duplicating group has found increasing advantages from its new $17 \times 22$ offset press, some of 


\begin{tabular}{|c|c|c|c|}
\hline \multicolumn{4}{|c|}{ Table 9} \\
\hline & $\begin{array}{l}\text { Fiscal } \\
1952\end{array}$ & $\begin{array}{l}\text { Fiscal } \\
1953\end{array}$ & $\begin{array}{c}\text { Fiscal } \\
1954\end{array}$ \\
\hline Offset pages & $2,610,415$ & $2,175,640$ & $3,819,809$ \\
\hline Offset plates & 2,469 & 1,454 & 1,125 \\
\hline Offset negatives & 4,681 & 3,672 & 4,445 \\
\hline Mimeo impressions & $1,886,400$ & $1,716,480$ & $1,702,165$ \\
\hline Stencils cut & 3,510 & 2,387 & 1,726 \\
\hline Stencils run & (not recorded) & 10,201 & 8,312 \\
\hline $\begin{array}{l}\text { Sheets collated } \\
\text { and bound }\end{array}$ & 769,775 & 589,065 & $1,324,089$ \\
\hline $\begin{array}{l}\text { Report copies } \\
\text { issued }\end{array}$ & 28,945 & 31,335 & 30,419 \\
\hline
\end{tabular}

which have contributed to a shortening of production time on reports.

\section{SCIENTIFIC COMPUTATION}

Two types of computational equipment in addition to ordinary desk calculators are now available to Brookhaven scientists. These are the Remington Rand 409-2R Electronic Digital Punch Card Computer at Brookhaven and the Univac located in New York City and operated by New York University for AEC contractors. The Univac, providing a means of solving lengthy and highly complex problems, became available in the summer of 1953 . Between the problems which can be handled efficiently by the Univac and those handled easily on a desk calculator lies a large problem area, for which no computational facilities were available to Brookhaven. The punch card computer was selected to provide a means of handling the majority of these problems. The computer with other punch card equipment was installed as part of a joint fiscalscientific computing project late in 1953. By April 1954, the computing apparatus had been reasonably debugged and was sufficiently stable to allow computing service to be made available to the scientific staff.

A Laboratory Computer Committee, whose membership consists of representatives from the various fields of science at the Laboratory and consultants from the fields of machine computation and electronics, was created to insure the most efficient use of all computing facilities available to the Laboratory. One of the principle functions of the Committee is to review requests for computation and
1) determine whether or not the problem should be placed on one of the electronic computers,

2) determine on which of the computers each problem could be handled most efficiently,

3) examine the physics and mathematics to ascertain whether or not portions of the problem may have been computed elsewhere, and suggest possible alternate analytical, physical or mathematical approaches.

The Committee formulates policies concerning the organization and operation of the centralized computing service with the policies subject to review by the Director's Office. The staff of the computing organization operating the local computer is directly responsible to the Committee for the execution of their part of the program.

The staff of the local computing organization is headed by a mathematician who supervises the computing operation, acts as consultant to the Committee and to the scientist requesting computation, provides programing and encoding and performs numerical analyses when needed. $\mathrm{He}$ and his assistant work closely with members of the tabulating section whose chief responsibilities lie in the fiscal area. All machines of the tabulating section are available to a greater or lesser degree for computation purposes. Although the majority of fiscal punch card handling operations take place on machines other than the computer, a small portion (20-25 percent) of computer time is required for its work. Joint operation of the punch card machines to satisfy the requirements of the Laboratory's fiscal operations and in the provision of a scientific computer service has been highly successful through the close cooperation of the personnel involved.

During the fiscal year 1954, the New York University Univac Computer staff provided the Laboratory scientists with a total of 807 hours of computation. The following is a breakdown of the total computing time by department:

\begin{tabular}{|c|c|c|}
\hline Accelerator Development & \multicolumn{2}{|c|}{38 percent } \\
\hline Nuclear Engineering & 32 & ", \\
\hline Reactor & 9 & $"$ \\
\hline Physics & 9 & " \\
\hline Chemistry & 7 & $"$ \\
\hline Cosmotron & 5 & $"$ \\
\hline
\end{tabular}

The local computing organization using the punch card equipment provided a total of approximately 130 hours (essentially all in the last three months) of computation, excluding program research and debug- 
ging time. Approximately 94 percent of the total time was used in computations for the Nuclear Engineering Department. The remaining 6 percent was for the Chemistry Department. A large amount of computer time has been used for program research, as would be expected in the early days of a newly formed computing organization. Debugging procedures for each newly formed program have occupied additional time. At each step a distinct effort has been made to provide programs which would handle not only the requested computations but any similar computations which might arise in the future. In addition, a library of sub-routines has been established and is constantly being enlarged. The growth of this organization's capacity for handling an increasing amount of computation has progressed rapidly with the acquisition of additional personnel, greater floor space and essential auxiliary equipment. 


\section{ADMINISTRATION AND OPERATIONS}

Reports on the personnel, business and operational activities of the Laboratory are included in this section. The personnel sub-section covers status and turn-over data for both the scientific and nonscientific staffs. Some of the broader aspects related to the scientific staff are discussed in the general introduction at the beginning of this report. The personnel statistics for this report period are very similar to those of the two preceding years; they reflect the fairly stable employment situation which exists. The figures, however, do not reflect shifting of personnel from areas where requirements were reduced to areas where new activities have necessitated the addition of personnel.

The activities of the Architectural Planning and Plant Maintenance Department involved direct technical support of the Laboratory's research program, as well as general operation and maintenance of the Laboratory plant. The work of the Receiving, Warehousing and Distribution group, the Purchasing Division, and Inventory Management has continued to provide the scientific staff with a greater variety and a more useful inventory of materials and supplies at less expense to the Laboratory and to the government. Laboratory purchasing activity rose to a new high in the number of transactions effected.

A very impressive safety record was established. During the past year the Laboratory for the second time in its history qualified for the National Safety Council's award of merit.

The Security Division through a re-assessment of the physical security requirements was able to reduce the police manpower by 17 people during the report period. A wide variety of requests for security protection require the maintenance of a flexible staff.

\section{Scientific Staff}

\section{PERSONNEL}

The composition of the scientific staff on June 30 for the past three years is given in Table 1 . Research Associates, who hold appointments similar to those of postdoctoral fellows for a maximum of

Total scientific staff employed June 30, 1954, compared with June 30, 1952, and 1953

\begin{tabular}{|c|c|c|c|c|c|c|c|c|c|}
\hline \multirow{3}{*}{ Appointees } & \multirow{2}{*}{\multicolumn{3}{|c|}{ Regular Staff }} & \multicolumn{6}{|c|}{ Visitors } \\
\hline & & & & & Salaried & & & ompen & ion \\
\hline & 1952 & 1953 & 1954 & 1952 & 1953 & 1954 & 1952 & 1953 & 1954 \\
\hline \multicolumn{10}{|l|}{ By Appointment Category } \\
\hline Senior scientist & 33 & 34 & 34 & 5 & 10 & 1 & 2 & 6 & 10 \\
\hline Scientist & 29 & 42 & 61 & 5 & 12 & 5 & 16 & 21 & 41 \\
\hline Associate scientist & 153 & 141 & 148 & 12 & 15 & 19 & 20 & 27 & 44 \\
\hline Research associate & - & 一 & - & 13 & 9 & 17 & 4 & 3 & 4 \\
\hline Junior scientist & 30 & 29 & 28 & 1 & - & 4 & 2 & 7 & 4 \\
\hline Jr. research associate & - & - & - & 7 & 15 & 13 & $26^{*}$ & $18^{*}$ & $23^{*}$ \\
\hline Research assistant & 2 & 1 & 1 & 63 & 71 & 88 & 1 & 5 & 16 \\
\hline TOTAL & 247 & 247 & 272 & 106 & 132 & 147 & 71 & 87 & 142 \\
\hline \multicolumn{10}{|l|}{ By Academic Degree } \\
\hline Ph.D. or M.D. & 152 & 152 & 164 & 32 & 44 & 47 & 31 & 52 & 73 \\
\hline Master & 36 & 34 & 31 & 11 & 19 & 25 & 15 & 7 & 31 \\
\hline Bachelor & 54 & 56 & 72 & 38 & so & 50 & 25 & 27 & 35 \\
\hline No Degree & 5 & 5 & 5 & 25 & 19 & 25 & - & 1 & 3 \\
\hline
\end{tabular}


Table 2

Classification of visiting scientists participating in

BNL program for period of one month or more

June 1, 1953 - May 31, 1954

\begin{tabular}{|c|c|c|c|c|c|c|}
\hline & \multicolumn{2}{|c|}{ More than 3 months } & \multicolumn{2}{|c|}{ Less than 3 months } & \multicolumn{2}{|c|}{ Total } \\
\hline & Salaried & Guest & Salaried & Guest & Individuals & Institutions \\
\hline University staff & 13 & 10 & 43 & 29 & 95 & 42 \\
\hline Thesis students & 16 & 4 & 1 & 3 & 24 & 10 \\
\hline Student helpers & - & - & 71 & 5 & 76 & 46 \\
\hline Special students & - & 1 & - & 29 & 30 & 10 \\
\hline TOTAL & 29 & 15 & 115 & 66 & 225 & 64 different \\
\hline Industry & 1 & 4 & - & 11 & 16 & 9 \\
\hline Other institutions & 2 & 18 & - & 21 & 41 & 23 \\
\hline Total & 32 & 37 & 115 & 98 & 282 & 96 different \\
\hline
\end{tabular}

two years, are included for statistical purposes under the heading of "visitors." During the past year there were 49 additions to the regular staff and 24 terminations, a net increase of 25 , mostly in the new Department of Accelerator Development. The number of both salaried and nonsalaried visitors has also increased, the total of the two being 32 percent higher than on June 30, 1953.

The continued increase in the participation of individuals from other institutions in the work of the Laboratory is gratifying. A detailed analysis of this participation is given in Table 2. The review period of June 1, 1953, through May 31, 1954, has been selected in order to include information on one summer program only, that of 1953. Under two groupings, the table gives the number of salaried and nonsalaried (guest) scientists who participated in the Laboratory program. The first group includes those scientists who worked at the Laboratory for more than three months and the second includes those who worked at the Laboratory from one to

Table 3

Consultants' services

\begin{tabular}{lrrr}
\hline \multicolumn{1}{c}{ Item } & \multicolumn{3}{c}{ Fiscal Year } \\
& 1952 & 1953 & 1954 \\
\hline Total contracts in effect June 30 & 95 & 94 & 94 \\
No. of consultants used & 51 & 48 & 54 \\
No. of man-days of service & 691 & 466 & 580 \\
\hline
\end{tabular}

three months. Scientists who worked at the Laboratory on a continuous or an intermittent basis for less than one month (about 35 in number) are not included in this summary.

There were 282 different individuals from 96 different institutions who participated in the program for one month or more during the twelve-month period indicated. The number of scientists from educational institutions increased by 21 percent over that of the previous year. While there was a decrease of 13 in the number of visitors who worked at the Laboratory for more than three months, an increase of 56 , or 36 percent, occurred in the number who worked here from one to three months. Twenty-four graduate students were engaged in their doctoral investigations; six of them completed their theses.

The number of representatives from nonacademic organizations also increased slightly over that of the previous year.

The man-days of service rendered by consultants are shown in Table 3 in which comparisons are made between the current report year and the two previous fiscal years.

\section{Summer Program for 1954}

About 85 faculty members from about 40 different educational institutions plan to work at the Laboratory for periods up to three months during the summer of 1954.

For the third successive year, a summer program has been arranged for students. Announcements were sent in January to about 100 educational institutions 
in the northeastern region. Applications were received from 354 students representing 89 institutions. Of the 354, 262 were undergraduate and 92 graduate students. Ninety-eight appointments were offered, of which 88 have been accepted -45 undergraduate and 43 graduate students representing 44 different educational institutions.

Including the Radiological Physics Fellows and additional graduate students brought here by faculty members to work as their research assistants, the scientific staff will be increased during the summer months by about 200 additional workers - a larger influx than in any previous year.

\section{Employment}

The number of Laboratory employees on June 30 , 1954, totaled 1446, representing a net increase of 15 staff members during the fiscal year. This augmentation of personnel only partially reflects the trend of continued moderate growth of the scientific staff, with a concomitant reduction in the ratio of scientific to nonscientific staff members. The ratio of 1 scientist to 3.7 nonscientists which existed on June 30, 1954, represents the lowest proportion of this type in the history of Laboratory operations. Pertinent personnel statistics are set forth in Table 4.

The similarity of the data between the last two fiscal periods, which may be observed from the above table, are suggestive of the relatively stable employment situation at the Laboratory. The data for the past year, however, do not reflect entirely the upsurge that has been experienced in employment and placement activity occasioned by the combined effect of reduced personnel requirements in certain areas, increased personnel demands in other existing areas, and the inauguration of new activities. Notable in this connection have been such activities as the placement in other organizational units of the Laboratory of a relatively large group of employees from the Fiscal Division who became surplus in that Division as a result of the mechanization of the accounting system, and recruitment efforts required for the Alternating Gradient Synchrotron Project and for the Accelerator Development Department. Although the labor market presumably has "eased," this phenomenon is not clearly evident in the recruitment of specialized technical personnel, or of employees in various categories of highly skilled design or shop work; the procurement of such employees occupies a relatively large proportion of the total effort expended by the Employment group. One may note that at the close of the fiscal year both the number of "open" requisitions (i.e., requisitions for which personnel have not yet been selected) and "pending" requisitions (i.e.,. requisitions for which personnel have been selected tentatively but have not yet commenced employment) were approximately double those which existed at the close of the preceding fiscal period.

\section{Labor Relations}

Maintenance and Service Employees: The collective bargaining contract negotiated with Federal Labor Union No. 24426, AFL, on July 21, 1951, which covers maintenance and some service employees, extends until June 30, 1955, without any mandatory reopening provisions. A series of conferences between the Union and the Laboratory for modifying certain portions of this agreement on a mutually voluntary basis did not result in any contract change. However, agreement was obtained on application of

\begin{tabular}{|c|c|c|c|c|c|c|}
\hline \multicolumn{7}{|c|}{ Table 4} \\
\hline Staff Data & & Fiscal 1953 & & & Fiscal 1954 & \\
\hline Total number of employees & & $1431 *$ & & & $1466 * *$ & \\
\hline Turnover Data & $\begin{array}{c}\text { Total } \\
\text { Numbers }\end{array}$ & $\begin{array}{c}\text { Annual } \\
\text { Rate }(\%)\end{array}$ & $\begin{array}{l}\text { Average } \\
\text { Monthly } \\
\text { Rate }(\%)\end{array}$ & $\begin{array}{c}\text { Total } \\
\text { Numbers }\end{array}$ & $\begin{array}{c}\text { Annual } \\
\text { Rate }(\%)\end{array}$ & $\begin{array}{l}\text { Average } \\
\text { Monthly } \\
\text { Rate (\%) }\end{array}$ \\
\hline Accessions & 227 & 16.14 & 1.35 & 231 & 16.17 & 1.35 \\
\hline Separations (turnover) & 208 & 14.88 & 1.24 & 216 & 15.11 & 1.26 \\
\hline Net accessions & 19 & 1.28 & 0.11 & 15 & 1.17 & 0.10 \\
\hline Replacements (net turnover) & 170 & 12.22 & 1.02 & 168 & 11.76 & 0.98 \\
\hline
\end{tabular}


the cost-of-living clause since the Bureau of Labor Statistics reference base set forth in the contract for determining the periodic adjustment no longer was available from the Bureau. The essential nature of the new agreement provides for a continuation of the cost-of-living adjustment on the same basis as set forth in the contract, determination thereof being made by an appropriate conversion formula from the "new" index to the "old" index as provided by the Bureau of Labor Statistics. The nature of the relationship between this Federal Labor Union and the Laboratory during the past year is best evidenced by the limited use of the grievance procedure at its terminal limits. Only four grievances were heard at the fourth step of the five-step grievance procedure provided in the contract and no grievances were processed to the fifth step, which provides for arbitration on a limited number of issues.

Patrolmen: The collective bargaining contract negotiated with the United Security Employees Union on May 4, 1953 extends until September 30, 1954. Although this Union during the past year indicated its intention of considering affiliation with another union organization, most recent advice indicates their decision to "carry on" within the framework of the United Security Employees Union. An unfair labor practice charge against the Laboratory by a member of this Union, which followed a necessary reduction in force among the patrolmen, was withdrawn after a preliminary hearing by a National Labor Relations Board Examiner. The essential nature of the relationship between this Security Employees Union, as with the Federal Labor Union, and the Laboratory also is best evidenced by the limited use of the grievance procedure. No grievance was processed as far as the third step of a four-step grievance procedure provided in the collective bargaining contract.

\section{Wage and Salary Administration}

The periodic wage and salary survey did not indicate the need for any essential modification of the wage and salary schedule. However, in accordance with Laboratory policy, the performance and progress of personnel were examined at the semi-annual reviews and appropriate adjustments in accordance with existing ranges were made on a merit basis.

As a result of organizational changes, it was necessary to establish several new jobs and eliminate others in the wage schedules. This was particularly evident in the Fiscal Division in connection with the mechanization of the accounting system. Also, in accordance with existing policy, a periodic audit of all job classifications was begun during the past year in order to assure that job descriptions properly reflect work actually being performed and that the related evaluation appropriately rates the job within the established labor grade structure.

\section{Employee Benefit Programs}

Participation in the hospitalization and group insurance programs by more than 80 percent of the staff has continued. Participation in the retirement plan continues to increase markedly each year, approximately 70 percent of the staff participating therein on June 30,1954 . In keeping with the policy of assuring the adequacy and appropriateness of the various benefit programs, a detailed study in relation thereto was undertaken and completed during the past fiscal year.

\section{Policy Modifications, Revisions and Supplements}

Essential modifications to the Manual of Personnel Policies have been made in the addition of new jobs and deletion of other jobs from the wage and salary schedules in accordance with modified operational requirements. The Manual of Personnel Policies, which also constitutes the basic document of Reimbursement Authorization with the Atomic Energy Commission, continues to serve effectively in its role as a working guide for supervisors and an orientation device for new personnel as well as a convenient mechanism for handling reimbursement requests with the Commission.

\section{Selective Service}

Although some changes in the Selective Service situation have been evident, the Laboratory continues to receive excellent cooperation from local draft boards. The previously established practice of notifying local boards whenever an employment offer has been accepted by a draft-eligible technical or scientific employee, and subsequently advising the boards of an individual's contribution to the Laboratory program after employment has begun, is considered primarily responsible for the Laboratory's success in obtaining required deferments. However, with recent modifications in Selective Service policy, notifications have been received from several local boards which indicate that in all probability no further deferments from military service will be issued 
at the local level in those cases where several deferments already have been granted.

\section{Employee Activities}

The Brookhaven Employees Recreation Association continues to sponsor some 20 social, cultural and physical activities which function without direct subsidy from the Laboratory. The most active groups this past year have been the Theatre Group, the Concert Group, the Camera Club and the Bowling League; the League had 32 teams.

The power-tool rental service, inaugurated last year, has proved very popular and the list of tools available to employees has grown appropriately. In addition, during the past year BERA has undertaken the purchase of ten automatic soft drink vending machines which have been placed about the site.

\section{Residence Services}

The off-site apartments at Stony Brook, L. I., remain fully occupied by "on-leave" and "term" appointees. Summer usage of on-site apartments again is scheduled for maximum occupancy; the winter occupancy of the on-site apartments remains high with the usage of such apartments by dormitory groups during the "off-season." The summer of 1954 is scheduled to be the largest to date from the standpoint of on-site residents. The demand for additional dormitory room assignments over previous years has been particularly evident.

The regular annual survey to determine rents charged for private housing facilities in nearby communities was prepared in January 1954. The survey did not reveal any appreciable shift in the rents for off-site apartment facilities. The cost of such facilities continues to be comparable to charges for similar accommodations provided by the Laboratory and no need for modifiation of the rent structure for Laboratory apartment units was indicated. However, some trend toward a moderate up-swing in single room rates was observed. After consideration of all the factors involved, a moderate increase in the rental structure for dormitory and guest house rooms was announced on June 1, 1954.

\section{Safety Engineering}

The disabling injury frequency and severity rates for the fiscal year. ending June 1954, and the cumulative rates for the immediately preceding five. year period, are recorded in Table 5.
Table 5

\begin{tabular}{lcc}
\multicolumn{3}{c}{ Disabling injury rates } \\
\hline \multicolumn{1}{c}{ Rate } & $\begin{array}{c}\text { Fiscal Year Ending } \\
\text { June 1954 }\end{array}$ & $\begin{array}{c}\text { Five-Year Period } \\
\text { July '48 - June '53 }\end{array}$ \\
\hline Frequency* & 2.64 & 2.56 \\
Severity** & .036 & .038 \\
\hline
\end{tabular}

* Number of disabling injuries per million man-hours worked. **Days lost per thousand man-hours worked.

The primary objective of the Safety Engineering group is to maintain the level of safety performance that has been achieved. The data for the past fiscal year shows a continued frequency and severity rate below the general average achieved in all AEC operations for the calendar year 1953, and well below the average frequency for the chemical industry - the nearest comparable industrial operation - for the same period.

During the past fiscal year the Laboratory qualified, a second time, for the National Safety Council's "Award of Merit" - thus adding to its collection of safety honors which already includes the Award of Merit presented by the National Safety Council in 1952, the National Safety Council's Distinguished Service to Safety Award bestowed in 1950, and the AEC Safety Trophy awarded in 1952.

Tangible evidence of the long range effects of the Laboratory safety program continues to be seen, at least in part, in the amount of adjustments received each year from the compensation and liability insurance carrier under the terms of a retrospective rating insurance plan. Although these adjustments are subject to revision, the net effect has been a savings in insurance costs alone, on Laboratory operations, amounting to more than 88 percent of the estimated standard insurance premium that might have been anticipated in the absence of a properly functioning safety program.

Major steps were taken in the past year toward attaining a state of "improved risk" fire protection at Brookhaven National Laboratory. In this connection, the operational requirements of the AGS project dictated that the Laboratory re-examine its position on the ultimate disposition of some facilities that were provided with less than optimum fire protection. As a result, the installation of an automatic spray sprinkler system in the structure housing the 
electron analogue of the alternating gradient synchrotron project was undertaken. This installation marks the first use by any AEC contractor of spray heads, the most important advance in sprinkler head design in many years.

Approximately $\$ 40,000$ was allocated for fire protection installations during the past fiscal year. The fire loss ratio for the fourth consecutive year has continued at nil, although the assessed property valuation has risen to over $\$ 57,000,000$. This record may be compared to a ratio of $\$ .004$ per $\$ 100$ valuation for all AEC operations for the calendar year ending December 1953 , and a ratio of $\$ .03$ per $\$ 100$ of valuation for "improving risk" facilities in industry as a whole over a prolonged time period.

During the past year the Safety Engineering group assisted the members of the National Fire Protection Association Committee on Atomic Energy in the preparation of a suggested code establishing fire protection requirements for radiation laboratories.

\section{SECURITY AND PLANT PROTECTION}

Activities of the Security and Plant Protection Division may be divided into Security, Police and Fire operations for purposes of evaluation.

The Security office is primarily concerned with the problem of security clearance of personnel, the processing of visitors, the control of entry into and exit from restricted areas and the site itself and the administration of the affairs of the Police and Fire groups.

A statistical analysis of the Security office records provides some indication of the volume of detail re-

\begin{tabular}{|c|c|c|c|}
\hline \multicolumn{4}{|c|}{ Table 6} \\
\hline & $\begin{array}{l}\text { Fiscal } \\
1952\end{array}$ & $\begin{array}{l}\text { Fiscal } \\
1953\end{array}$ & $\begin{array}{l}\text { Fiscal } \\
1954\end{array}$ \\
\hline Division personnel & 118 & 116 & 95 \\
\hline Regular visitors processed & 15,803 & 17,667 & 13,197 \\
\hline Conference visitors processed & 1,022 & 2,555 & 2,017 \\
\hline $\begin{array}{l}\text { Sub-contractor's employees } \\
\text { processed }\end{array}$ & (1) & (1) & $7,967(2)$ \\
\hline Alien visitors processed & 292 & 396 & 553 \\
\hline Total visitors & 17,117 & 20,618 & 23,734 \\
\hline Clearances processed & 1,283 & 1,235 & 739 \\
\hline
\end{tabular}

(1) Included under regular visitors

(2) December to June only. quired of the Security office in complying with AEC requirements and the policies and procedures of the Laboratory.

In 1953 the Atomic Energy Commission's revision of certain security requirements permitted the Laboratory to eliminate several regularly scheduled police posts. As a result, police manpower was reduced approximately 26 percent December 4, 1953.

Approximately 5,445 hours were expended to cover special posts and details compared with approximately 9,000 hours during the previous year. These special assignments occur constantly on a nonscheduled and sometimes emergency basis so that the need for the size of the group over and above fixed post requirements must be judged on the basis of anticipated and yet unknown factors.

Fire group activity showed a marked increase over the previous year in all phases of work. The group has shown a consistently good record not only in the quality of its fire fighting techniques but also in its prevention program. During the last quarter of the year personnel of the Medical Department, Health Physics Division and Hot Laboratory were given practical instruction in the proper use of various types of extinguishers and chemox breathing apparatus. This type of personal contact is a part of the fire prevention program which continues throughout the year in conjunction with the testing of fire fighting equipment.

Statistical record of activities is as follows:

$\begin{array}{lc}\text { Fire Group } & 1953-54 \\ \text { Responses on site } & 75 \\ \text { Responses off site } & 3 \\ \text { No response - investigation } & 18 \\ \quad \text { Total alarms } & 96\end{array}$

Causes of alarm on site were established in all cases. Electrical and equipment failures were generally responsible and unpreventable. Careless smokers accounted for six fires and children playing with matches accounted for one. There were no major fires during the fiscal year.

\section{ARCHITECTURAL PLANNING AND PLANT MAINTENANCE}

For the first time in four years the personnel strength of the Architectural Planning and Plant Maintenance Department showed no marked change during FY 1954 (244 at July 1, 1953; 243 at June $30,1954)$. The personnel level of the Buildings and 
Grounds Division, including supervisory and administrative personnel, remained at 220 throughout the entire year.

An analysis of the work order system instituted two years ago in the Buildings and Grounds Division indicates the manner in which its effort was distributed in both FY 1953 and FY 1954. Comparison between the two years shows that significant changes occurred in three categories:

a) The sharp decrease of 15.8 man-years in "heating and ventilating" is the direct result of the Rochester Avenue steam line extension and the completion of the program of conversion to oilburning units; this decrease represents a net reduction in expenditure of more than $\$ 65,000$ per year.

b) The increase in the item "protective clothing service" can be ascribed to the more frequent and higher level contamination which occurred at the reactor and in the reactor canal; it appears that this trend is likely to continue.

c) The increase in the item "technical services" shows that a greater portion of the total effort of the Division is being contributed directly to the scientific departments in the way of construction, maintenance and repair of apparatus and equipment as well as in routine maintenance repairs and operations of certain parts of the scientific facilities.

In connection with the last item, it is of interest to note that, as was the case last year, three of the crafts (electricians, plumbers, cabinet makers) which represent about 21 percent of the total effort contributed in FY 1954 almost half (48 percent) of the technical services rendered by the Division.

The following are jobs in progress and completed during the report period.

A. Construction completed by contractors. These new facilities were added during the year:

1. Cosmotron shielding enclosure $\sim \$ 16,000$

2. Counting room for Chemistry complex 25,000

3. Decontamination facility at canal house 20,000

4. Experimental dc $\mathrm{m}-\mathrm{g}$ house for

Cosmotron

100,000

In addition there were more than $\mathbf{4 5}$ smaller jobs involving changes to and extensions of various laboratory services and equipment such as piping, electrical work, air conditioning, laboratory furniture, shielding blocks, fume hoods, sprinklers, experiment hook-ups, emergency electrical power m-g sets, as well as approximately 20 jobs concerned with physical improvements to and maintenance of buildings (exterior and interior painting, re-roofing, insulation, sash repair, etc.) and approximately six jobs in the category of additions to and modifications of sitewide facilities (electric power lines, sewage lines, grading, and paving).

B. Planning and Design (approximately 100 jobs, excluding operational and maintenance engineering):

1. Major planning work:

a. Collaboration with AGS project in the consideration of initial location and layout studies as well as assistance in preparing preliminary estimates for buildings and site services.

b. Preparation of preliminary architect-engineer studies and establishing of basic physical requirements for the proposed Medical Research Center including cost estimates of several schemes for budget purposes. This was followed by active collaboration with the architect-engineer firm of Eggers and Higgins, New York, during the course of the performance of their work under subcontract with AUI-BNL to further evaluate, refine and report on the proposed Medical Research Center as a final step to budget proposals.

c. Preliminary architect-engineer studies for $\mathrm{Nu}$ clear Engineering Laboratory adjacent to Hot Laboratory.

2. Complete architect-engineering services for new facilities and improvements were furnished for:

a. Approxumately 50 jobs involving additions, improvements and/or modifications of existing space in various buildings to provide new or improved facilities such as: Chemistry counting room; Cosmotron $\mathrm{m} \cdot \mathrm{g}$ house; decontamination facility at reactor; Cosmotron shield addition; payroll and computer room modifications; shielding blocks for Cosmotron and others; laboratories for alpha work and reactor chemistry in the Hot Laboratory; sprinkler systems in the Cosmotron test shack, central and maintenance stockrooms; new platform and added power at the reactor; rehabilitation of six buildings to house AGS project including new services; installation of $350 \mathrm{kw} \mathrm{m-g}$ sets at reactor, Hot Laboratory and Metallurgy Laboratory to provide emergency power; ce- 
ment handling equipment of use in disposal of contaminated wastes at the waste concentration plant; structural, mechanical and electrical work for AGS electron analogue.

b. About 100 minor improvements concerning changes to or extensions of existing laboratory services which involved electrical work, piping ventilation, air conditioning, dehumidification, laboratory furniture, fume hoods and other miscellaneous improvements to existing buildings and separate laboratories.

c. Approximately 20 jobs concerned with services and improvements of the Laboratory site such as electric voltage regulation station for the Medical area; minor changes in electric, steam and water distribution systems; up-dating of utilities and topographic maps.

\section{BUSINESS OPERATIONS}

\section{Fiscal}

In fiscal year 1954 a major change was made in accounting methods and procedures in the Laboratory. As reported previously, a decision to adopt punch card accounting had been made at the end of the 1953 fiscal year. Throughout the summer and autumn of 1953 employees of the Fiscal Division together with representatives of Remington Rand, Inc., whose equipment had been selected for the work, developed procedures for punch card accounting preparatory to converting to the new system January 1, 1954. Delivery of equipment was completed late in December and after a short testing period the machine was placed in operation the beginning of January.

In the month of January material and supplies inventory records, accounts payable, and all payroll work were placed on punch card equipment. In the month of March all bookkeeping with the exception of the general ledger work was placed on the punch card system. Plans are presently being made to convert personnel and fixed asset records to the Remington Rand card equipment during fiscal year 1955.

As stated earlier in this report, the electronic computer and its related punch card equipment is considered as a Laboratory service installation for the purpose of providing scientific computations for the research programs as well as statistical and accounting information for management. Experience to date indicates that this arrangement has been and will continue to be an effective and economical service to the Laboratory. It is expected that the scientific research programs will require approximately 75 percent of the electronic computer time but that the development of accounting and statistical information will require the major portion of the time of the related equipment.

During the fiscal year audits of the Laboratory fiscal operations were performed by Atomic Energy Commission Audit Branch, General Accounting Office, and Haskins \& Sells, Certified Public Accountants.

The personnel level of the Fiscal Division at June 30,1954 , stood at 38 as compared with 54 at June 30,1953 . The reduction in employees resulted from the conversion to punch card accounting. Although there will be substantial economy effected by the conversion, the reduction of personnel reflected above cannot be regarded as a net saving in view of the fact that machine rental costs of approximately $\$ 30,000$ per year have been incurred.

\section{Inventory Management}

Efforts were continued to dispose of inventory items in excess of the Laboratory's needs and maintain the active inventory at levels that would satisfy the Laboratory's demands. The results of these efforts are reflected in Tables 7 and 8 . The number of months' investment in active inventory at June 30 has been:

$\begin{array}{cccc}1951 & 1952 & 1953 & 1954 \\ 14 \text { months } & 10 \text { months } & 7.9 \text { months } & 6.5 \text { months }\end{array}$

As a phase of the Laboratory's program of disposition of excess, donations amounting to $\$ 36,400$ of book value were made to other Federal agencies and to educational institutions. These donations were made in accordance with the government's program

Table 7

Disposition of excess inventory

\begin{tabular}{lccc}
\hline & \multicolumn{3}{c}{ Fiscal Year } \\
& 1952 & 1953 & 1954 \\
\hline $\begin{array}{l}\text { Book values of excess } \\
\text { inventory sold/donated }\end{array}$ & $\$ 77,400$ & $\$ 202,300$ & $\$ 141,308$ \\
$\begin{array}{l}\text { Receipts from sales } \\
\begin{array}{l}\text { Percent of book } \\
\text { value recovered }\end{array}\end{array}$ & 33,700 & 110,700 & 17,716 \\
\hline \hline
\end{tabular}


Table 8

Inventory changes

\begin{tabular}{|c|c|c|c|}
\hline \multirow{2}{*}{ Type of Inventory } & \multicolumn{3}{|c|}{ Fiscal Year } \\
\hline & 1952 & 1953 & 1954 \\
\hline \multicolumn{4}{|l|}{ Active Inventory } \\
\hline $\begin{array}{l}\text { Opening active inventory July } 1 \\
\text { Net of purchases, stores issues, adjustments, } \\
\text { additions to inventory, transfers to excess } \\
\text { Closing active inventory June } 30\end{array}$ & $\begin{array}{r}\$ 803,322 \\
-251,183 \\
552,139\end{array}$ & $\begin{array}{r}\$ 552,139 \\
-133,530 \\
418,609\end{array}$ & $\begin{array}{r}\$ 418,609 \\
-22,045 \\
396,564\end{array}$ \\
\hline \multicolumn{4}{|l|}{ Excess Inventory } \\
\hline $\begin{array}{l}\text { Opening excess inventory July } 1 \\
\text { Net of transfers from active inventory, } \\
\text { dispositions, and adjustments } \\
\text { Closing excess inventory June } 30\end{array}$ & $\begin{array}{l}180,568 \\
131,287 \\
311,855\end{array}$ & $\begin{array}{r}311,855 \\
-157,657 \\
154,198\end{array}$ & $\begin{array}{c}154,198 \\
-154,198 \\
0\end{array}$ \\
\hline \multicolumn{4}{|l|}{ Total Inventory } \\
\hline $\begin{array}{l}\text { Opening total inventory July } 1 \\
\text { Net of purchases, stores issues, adjustments, } \\
\text { transfers to excess and dispositions } \\
\text { Closing Total Inventory June } 30\end{array}$ & $\begin{array}{r}983,890 \\
-119,896 \\
\$ 863,994\end{array}$ & $\begin{array}{r}863,994 \\
-291,187 \\
\$ 572,807\end{array}$ & $\begin{array}{r}572,807 \\
-176,243 \\
\$ 396,564\end{array}$ \\
\hline
\end{tabular}

to obtain maximum usage of all useable materials, and were undertaken in cooperation with General Services Administration and Public Education Administration.

Late in the fiscal year, the Laboratory, in cooperation with AEC, undertook a program to reduce handling costs and accounting costs pertinent to low cost items. The program includes some expansion of longer term buying of stable items, buying in package units consistent with manufacturers' or vendors' packaging custom, and charging to common-usage accounts under controlled conditions specified quantities of low unit-cost items. It is anticipated that this program will be completed during fiscal year 1955 .

\section{Receiving, Warehousing and Distribution}

During the past year, the personnel strength of the Receiving, Warehousing and Distribution group has remained at the constant level of 44 . During the 12 -month period just ended a total of 74,500 stores issues were written, a rate of more than one every two minutes per working hour; these issues for stockroom materials covered more than 145,000 separate items. These figures compare with corresponding totals in the previous year of 57,000 stores issues and 118,000 separate items. Rates of issues have climbed steadily during this last fiscal year to almost 7,000 per month, a rate more than double that of June 1952.

Figure 1 charts the issues from stores by quarters during the past two years and shows the continuing trends in all three categories of service rendered: a) Over the counter issues from Central Stores; b) issues from Central Stores delivered to users; c) issues from branch stockrooms. The relative importance of these three categories for the past two years is as follows:

$\begin{array}{llr}\text { Counter issues-central stores } & 43 \% & 40 \% \\ \text { Deliveries from central stores } & 33 \% & 35 \% \\ \text { Branch stockrooms } & 24 \% & 25 \%\end{array}$

Thus, it is evident that approximately 60 percent of all stores issues are now made available to the users at or close to the using points by delivery or by issues from branch stockrooms.

Under the direction of Inventory Management and Purchasing, the Receiving, Warehousing and Distribution group took part in the disposition of excess inventories totalling approximately 5500 items with a book value of almost $\$ 150,000$; the movement of this excess was a major undertaking. In collaboration with Inventory Management and for the purpose 


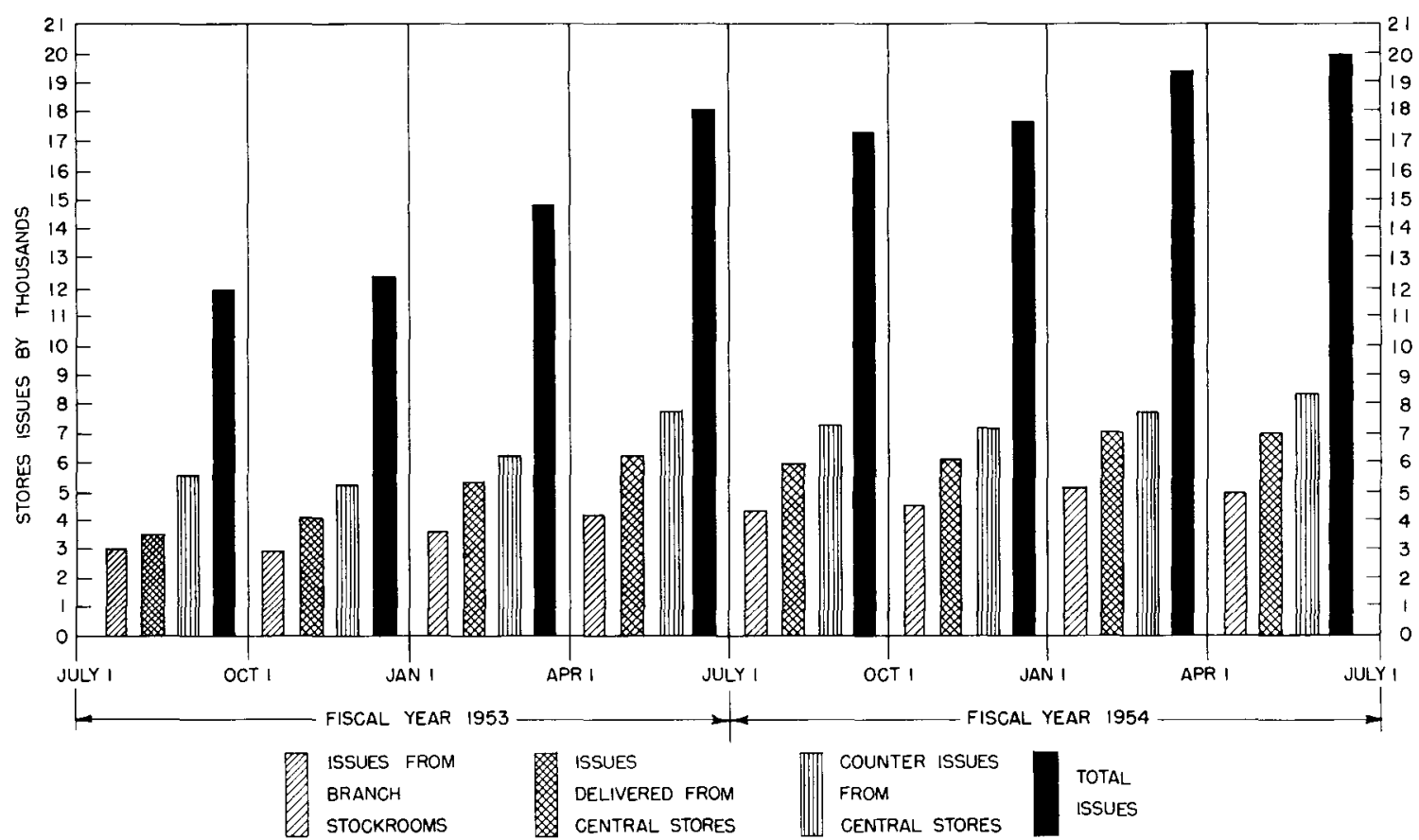

Figure 1.

of achieving better use and disposition of items of obsolete design or beyond economical repair, a program of general clean-up and re-arrangement of warehouse storage of capital equipment was initiated.

In preparation for the punch card accounting system which went into operation on January 1 , 1954, the nomenclature, description and stock numbers of the more than 17,000 items in the active inventory were revised to accommodate the requirements of the new system.

\section{Purchasing}

Fiscal year 1954 saw the Purchasing Division receive the greatest number of Requisitions and write the greatest number of Purchase Orders in the Lab. oratory's history. More than 14,300 Requisitions were received and nearly 16,000 Purchase Orders and Change Orders were written for a total dollar value of $\$ 2,513,546.41$. This total dollar figure is exceeded only by fiscal years 1948 and 1949. A tabulation showing the complete summary of operations from February 1947 through June 30,1954 is given in Table 9.

The Purchase Order form which provides copies for Receiving Reports continues to demonstrate its worth. Other AEC contractors have indicated their interest in adopting a similar system.
Considerable efforts during the past year have been devoted to making use of Excess Lists from other AEC contractors. As a consequence, approximately $\$ 200,000$ of new or slightly used equipment and material was acquired for a cost to the Laboratory of about 38 percent of original value plus transportation. These acquisitions included such items as the 20-ton crane, approximately 20 suburban carryalls, a fuel truck, three truck tractors, a carload of electronics and maintenance materials from Idaho Falls, a mass spectrometer, approximately 80 typewriters and such items as calculators, lead sheet, electronic instruments and even grass seed.

The Purchasing Division during the past year conducted eleven Excess Sales which were advertised in newspapers and notices of which were mailed to approximately 200 firms and individuals on each of the sales. The average number of bids received was 20. These public sales included such items as typewriters, a crane, tractors, autos, trucks, electronics, glassware and diesel generator sets. The dollar return in nearly all cases was quite satisfactory to the Laboratory. In addition, donations of excess materials approximating $\$ 36,400$ in book value were arranged in cooperation with government agencies.

The increased activity in the Purchasing Division required no increase in personnel. It is anticipated, 
Table 9

Record of purchase orders from February 1947 to June 1954

\begin{tabular}{|c|c|c|c|c|c|c|c|}
\hline Fiscal Year & $\begin{array}{c}\text { No. } \\
\text { P.R.'s }\end{array}$ & $\begin{array}{l}\text { No. } \\
\text { P.O.'s }\end{array}$ & Amount & $\begin{array}{c}\text { No. } \\
\text { C.O.'s }\end{array}$ & Deductions & Additions & Total Amount \\
\hline Feb. 47 to June 47 & & 2,195 & $\$ 938,229.03$ & 217 & $\$ 30,670.58$ & $\$ 6,390.06$ & $\$ 913,948.51$ \\
\hline July 47 to June 48 & & 10,695 & $3,939,419.42$ & 1,998 & $140,771.71$ & $94,536.49$ & $3,893,184.21$ \\
\hline July 48 to June 49 & & 11,588 & $4,158,103.61$ & 1,963 & $62,876.91$ & $81,391.93$ & $4,176,618.63$ \\
\hline July 49 to June 50 & & 11,548 & $1,985,286.38$ & 1,499 & $68,341.57$ & $417,546.61$ & $2,334,491.42$ \\
\hline July 50 to June 51 & 10,887 & 11,333 & $2,365,799.44$ & 1,442 & $41,552.79$ & $94,177.97$ & $2,418,424.62$ \\
\hline July 51 to June 52 & 10,816 & 11,019 & $1,900,129.69$ & 1,647 & $57,436.44$ & $79,442.46$ & $1,922,135.71$ \\
\hline July 52 to June 53 & 10,890 & 12,329 & $1,917,082.76$ & 1,553 & $63,714.24$ & $68,714.29$ & $1,922,082.81$ \\
\hline July 53 to June 54 & 14,304 & 14,362 & $2,509,376.63$ & 1,615 & $71,828.43$ & $75,998.21$ & $2,513,546.41$ \\
\hline
\end{tabular}

however, that a slight increase in staff will be required over the next several years to serve properly the expanding needs of the Laboratory.

\section{Office Services}

The Office Services section continued its established functions, including mail handling, telephone and telegraph service, the acquisition, issuance and maintenance of office machines and furniture, and the operation of archives.

Progress in records handling was aided by the addition of a second person in archives during the latter part of the fiscal year.

The typewriter replacement program progressed ahead of schedule through the acquisition of a number of nearly new machines from government surplus. Other business machines and office furniture were also obtained from this source. 


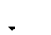




\section{A P P E N D I X}

\section{A. UNCLASSIFIED PUBLICATIONS, JULY 1, 1953 - JUNE 30, 1954}

This list includes official Laboratory publications, abstracts of papers which were or will be presented at scientific meetings, and publications by staff members, consultants and guests. All these listings result from work done at the Laboratory; they were submitted during the review period.* Abstracts are indicated by $(A)$; letters to the editor, (L); and notes, $(N)$. Acceptance for future publication is designated by (In press).

\section{GENERAL PUBLICATIONS}

Annual Report, July 1, 1953 (BNL 246 (AS-7))

Quarterly Progress Reports:

July 1 - September 30, 1953 (BNL 259 (S-19))

October 1 - December 31, 1953 (BNL $270($ S-20))

January 1 - March 31, 1954 (BNL 289 (S-21))

April 1 - June 30, 1954 (BNL 299 (S-22))

Conference Reports:

Brookhaven Symposia in Biology No. 6. Abnormal and

Pathological Plant Growth (BNL 258 (C-19))

Technical Report:

Integral Tables (BNL 257 (T-40))

Weekly Bulletin 6, No. 50-52; 7, No. 1-49

Weekly Selected Reading List 6, No. 18-52; 7, No. 1-17

\section{STAFF PUBLICATIONS AND ABSTRACTS}

\section{Biology Department}

Arnason, T. J. and Steffensen, D.

Frequency of chromosome aberrations produced by frac -

tional doses of $\mathbf{x}$-rays in Tradescantia

Genetics 39, 220-8 (1954)

Bard, R. C. (See DeMoss, R. D.)

Bard, R. C. (See Paege, L. M.)

Beevers, $H$. and Gibbs, $M$.

The direct oxidation pathway in plant respiration

Plant Physiol. (In press)

Beevers, $H$. and Gibbs, $M$.

Effect of the age of plant tissue on the pathway of glucose oxidation $(A)$

Plant Physiol.

Beevers, H. (See Gibbs, M.)

Beevers, $H$. and Gibbs, $M$.

Participation of the oxidative pathway in yeast respiration $(\mathrm{L})$

Nature 173, 640 (1954)

Beevers, $H$. and Gibbs, $M$.

Position of $\mathrm{C}^{\mathrm{t}}$ in alcohol and carbon dioxide formed from labeled glucose by corn root tips

Plant Physiol. (In press)

Boehm, R. F.

"Atomic" fruits for New Jersey growers

Horticultural News

*Also included are those listings from the last Annual Report (BNL 246 (AS-7)) for which complete reference information was not then available.
Bogner, R. L. (See Edelmann, A.)

Bowen, C. C. and Sparrow, A. H.

Radiosensitivity of several meiotic stages of lilium (A) Records Genetics Soc.

Budenstein, Z. (See Koshland, D. E., Jr.)

Caldecott, R. S., Beard, B. H. and Gardner, C. O.

Cytogenetic effects of $\mathrm{x}$-ray and thermal neutron irradiation on seeds of barley

Genetics 39, 240-59 (1954)

Caldecott, R. S.

Effects of ionizing radiation on seeds of barley (A)

Radiation Research Soc. Symposium, Cleveland, May, 1954

Caldecott, R. S.

The effects of $\mathrm{x}$-rays, 2-Mev electrons, thermal neutrons and fast neutrons on dormant seeds of barley N. Y. Acad. Sci.

Caspar, A. L. (See Singleton, W. R.)

Christensen, E.

An improved sealing wax for temporary smear preparations Stain Technology 29, 197-200 (1954)

Christensen, E. (See Sparrow, A. H.)

Christensen, E.

Root production in plants following localized stem irradiation Science 119, 127-8 (1954)

Clark, A. M.

The relation of genome number to radiosensitivity in habro. bracon (A) Radiation Research J.

Clarke, E. (See Koshland, D. E., Jr.)

Cochrane, V. W. (See Gibbs, M.)

DeMoss, R. D. (See Gibbs, M.)

DeMoss, R. D., Gunsalus, I. C. and Bard, R. C. Glucose-6-phosphate dehydrogenase in leuconostoc mesenteroides J. Bacteriol. 66, 10.6 (1953)

Deschner, E. E.

The relation of break sensitivity and rejoining in trillium following $\mathrm{x}$-irradiation (A) Records Genetics Soc. No. 22, 70 (1953)

Dollinger, E. J.

Studies on induced mutation in maize Genetics

Dougherty, E., 3rd, Saunders, L. Z. and Parsons, E. H. The pathology of infectious scrositis of ducks Am. J. Pathol.

Earl, J. M. (See Gibbs, M.) 
Edelmann, A., Bogner, R. L. and Steele, R. Anethole, sodium salicylate and glycogen deposition in the adrenalectomized fasted rat (A) Federation Proc. 13, No. 1 (1954)

Fluke, D. J.

Electron bombardment of $\mathrm{T}-1$ bacteriophage (A) Radiation Research J.

Forro, F. $\mathrm{P}^{32}$ distribution among the progeny of labeled bacteria (A) Records Genetics Soc.

Gibbs, M. and DeMoss, R. D. Anaerobic dissimilation of $\mathrm{C}^{\mathbf{1 4}}$-labeled glucose and fructose by Pseudomonas lindneri

J. Biol. Chem. 207, 689-94 (1954)

Gibbs, M., Paege, L. M. and Earl, J. M.

Anaerobic dissimilation of $\mathrm{C}^{14}$-labeled D-xylose and D-arabinose by Escherichia coli (A)

Bacteriol. Proc. p. 111 (1954)

Gibbs, M. (See Beevers, H.)

Gibbs, M

Effect of light intensity on the distribution of $\mathrm{C}^{14}$ in sunflower leaf metabolites during photosynthesis

Arch. Biochem. and Biophys. 45, 156-60 (1953)

Gibbs, M., Cochrane, V. W., Paege, L. M. and Wolin, H Fermentation of D-xylose-1- $\mathrm{C}^{14}$ by Fusarium lini $(\mathrm{A})$ Bacteriol. Proc. p. 110 (1954)

Gibbs, M., Cochrane, V. W., Paege, L. M. and Wolin, H. Fermentation of D-xylose-1. $C^{14}$ by Fusarium lini Bolley Arch. Biochem. and Biophys. 50, 237-42 (1954)

Gibbs, M. and Beevers, $\mathrm{H}$. Investigations on the path of carbohydrate breakdown in plants (A) Proc. 8th Intl. Botan. Cong.

Gibbs, M. (See Horecker, B. L.)

Gibbs, M. and Horecker, B. L.

The mechanism of pentose phosphate conversion to hexosemonophosphate. II. With pea leaf and pea root preparations J. Biol. Chem. 208, 813-20 (1954)

Gibbs, M. (See Paege, L. M.)

Gibbs, $M$.

The respiration of the pea plant. Oxidation of hexose phosphate and pentose phosphate by cell-free extracts of pea leaves J. Plant Physiol. 29, 34-9 (1954)

Gibbs, $M$.

Triosephosphate dehydrogenase from plant tissue D-glyceraldehyde-3-phosphate + PO + TPN (DPN) $\rightleftarrows 1,3$-diphosphoglyceric acid + TPNH (DPNH) Article for book Methods in Enzymology

Grau, C. R. and Steele, R.

Phenylalanine and tyrosine utilization in normal and phenylalanine-deficient young mice J. Nutrition 53, 59-71 (1954)

Gunckel, J. E. and Sparrow, A. H. Aberrant growth in plants induced by ionizing radiation Included in BNL 258, Symposia No. 6, 252-79 (1954)

Gunckel, J. E. (See Sparrow, A. H.)

Gunckel, J. E., Morrow, I. B., Sparrow, A. H. and Christensen, E. Variations in the floral morphology of normal and irradiated plants of Tradescantia paludosa

Bull. Torrey Botan. Club 80, 445-6 (1953)

Gunsalus, I. C. (See DeMoss, R. D.)

Heidenthal, G.

A comparison of $\mathbf{x}$-ray-induced dominant and recessive lethals in first meiotic metaphase eggs and in sperm of Habrobracon (A)

Records Genetics Soc. No. 22. 78 (1953)
Horecker, B. L., Gibbs, M., Klenow, H. and Smyrniotis, P. Z. The mechanism of pentose phosphate conversion to hexose monophosphate

J. Biol. Chem. 207, 393 (1954)

Horecker, B. L. (See Gibbs, M.)

Jakowska, S., Nigrelli, R. F. and Sparrow, A. H.

Local cell responses to induced skin infection in Triturus viridescens after whole-body irradiations (A) Radiation Research J.

King, R. C.

The calculation of radiation doses received by thermalneutron-treated Drosophila melanogaster Nucleonics

King, R. C. The effect of yeast on phosphorus uptake by Drosopbila Am. Naturalist 88, 155-8 (1954)

King, R. C.

Mutation in Drosopbila melanogaster males exposed to $\beta$ radiation from neutron-activated phosphorus-bakelite plaques Radiation Research J. 1, 369-80 (1954)

King, R. C. and Wood, E. M. Sex-linked lethal mutations induced by thermal neutrons in male and female Drosopbila melanogaster Genetics

King, R. C

Studies on Drosophila homogeneously labeled with radiophosphorus

J. Exptl. Zoology (In press)

King, R. C.

Studies with radiophosphorus in Drosophila. II. The effect of $\mathrm{P}^{32}$ - treatment upon development

J. Exptl. Zoology 125, 331 (1954)

Klein, J. R.

Effect of competitive inhibitors on the inactivation of $D$ amino acid oxidase by $\mathbf{x}$-rays (A) Federation Proc. 13, No. 1 (1954)

Klein, J. R. Inhibition of $\mathrm{D}$-amino acid oxidase by aromatic acids J. Biochem. 205, 725-30 (1953)

Kojan, S. (See Sparrow, A. H.)

Konzak, C. F.

The influence of oxygen tension on the genetic effects of $\mathrm{x}$-rays (A) Radiation Research J.

Konzak, C. F

Stem rust resistance in oats induced by nuclear radiation Agronomy J.

Koshland,. D. E., Jr. and Budenstein, Z. Cleavage of adenosinetriphosphate by actomyosin (A) Federation Proc. 13, No. 1 (1954)

Koshland, D. E., Jr. and Stein, S. S. Correlation of bond breaking with enzyme specificity cleavage point of invertase

J. Biol. Chem. 208, 139-48 (1954)

Koshland, D. E., Jr Mechanism of action of carbohydrases (A) Am. Chem. Soc. Meeting, September, 1954

Koshland, D. E., Jr. (See Stein, S. S.)

Koshland, D. E., Jr. and Clarke, E. Mechanism of hydrolysis of adenosinetriphosphate catalyzed by lobster muscle J. Biol. Chem. 205, 917-24 (1953)

Koshland, D. E., Jr., Budenstein, Z. and Kowalsky, A Mechanism of hydrolysis of adenosinetriphosphate catalyzed by purified muscle proteins J. Biol. Chem. (In press) 
Koshland, D. E., Jr.

A physical organic approach to enzymatic mechanism

Trans. N. Y. Acad. Sci. Ser. II, 16, 110-3 (1954)

Koshland, M. E.

$I^{131}$-labeled diphtheria toxoid and the rate of antigen elim-

ination in the guinea pig (A)

Federation Proc. 13, No. 1 (1954)

Kowalsky, A. (See Koshland, D. E., Jr.)

Mateyko, G. M. and Charipper, H. A.

Histological effects upon the pars anterior of the rat following hypophyseal cathode-ray irradiation and whole-body $x$ irradiation

J. Morphology 93, 533-59 (1953)

Mikaelson, $\mathrm{K}$

Protective properties of cysteine, sodium hyposulfite and sodium cyanide against radiation-induced chromosome aberrations

Proc. Natl. Acad. Sci. 40, 171-8 (1954)

Moses, M. J., Oliver, J., MacDowell, M. C. and Lee, Y. C. Cellular mechanisms of protein metabolism in the nephron. Il. The histochemical characteristics of protein absorption droplets J. Exptl. Medicine

Moses, M. J. and Taylor, J. $\mathrm{H}$.

Desoxypentose nucleic acid synthesis during microsporogenesis in Tradescantia (A)

Records Genetics Soc. No. 22, 88 (1953)

Nigrelli, R. F. (See Jakowska, S.)

Nims, L. F. and Sutton, E.

Adrenal cholesterol, liver glycogen, and water consumption of fasting and $\mathrm{x}$-irradiated rats Am. J. Physiol. 177, 51-4 (1954)

Nims, L. F. (See Scheraga, H. A.)

Paege, L. M. (See Gibbs, M.)

Paege, L. M., Gibbs, M. and Bard, R. C. Metabolism of $\mathrm{C}^{14}$-glucose by Clostridium perfringens (A) Bacteriol. Proc. p. 110 (1954)

Pond, V. (See Sparrow, A. H.)

Ponder, E., Ponder, R. V. and Anderson, R. C.

The combination between hemolysins and red cells or ghosts, as studied with a radioactive lysin and with new color reactions

J. Gen Physiol. 37, 411-22 (1954)

Ponder, R. V. (See Ponder, E.)

Renkin, E. M.

The effects of adrenal hormones on capillary permeability in perfused rat tissues

Am. J. Physiol.

Renkin, E. M.

Filtration, diffusion and molecular sieving through porous cellulose membranes J. Gen. Physiol.

Renkin, E. M.

Permeability and structure of inert porous membranes (A) Federation Proc. 13, No. 1 (1954)

Rubin, B. A.

Growth and mutation of bacteria during continuous irradiation

J. Bacteriol. 67, 361-8 (1954)

Sacks, J. and Sinex, F. M.

Insulin and the relation between phosphate transport and glucose metabolism

Am. J. Physiol. 175, 353-7 (1953)
Sand, S. A., Smith, H. H. and Sparrow, A. H.

Stimulation of chronic gamma irradiation of the spontaneous rates of heritable somatic instabilities in a clone of Nicotiana (A)

Genetics

Saunders, J. M. (See Scheraga, H. A.)

Saunders, L. Z. (See Dougherty, E., 3rd)

Saunders, L. Z. and Smith, R. F.

Progressive retinal atrophy in dogs

Photographie und Forschung 5, 225-7 (1953)

Schairer, L. A. (See Sparrow, A. H.)

Scheraga, H. A., Carroll, W. R., Nims, L. F., Sutton, E., Backus, J. K. and Saunders, J. M.

Hydrodynamic properties of urea-denatured fibrinogen

J. Polymer Sci.

Singleton, W. R.

The effect of chronic gamma radiation on endosperm mutations in maize

Genetics

Singleton, W. R. and Caspar, A. L.

Effects of time of gamma radiation on microspore mutation rate in maize $(A)$

Records Genetics Soc.

Sparrow, A. H. (See Gunckel, J. E.)

Sparrow, A. H., Kojan, S. and Pond, V.

Growth of excised anthers from Trillium erectum in various sterile culture media (A)

Proc. 8th Intl. Botan. Cong.

Sparrow, A. H. and Christensen, E.

Improved storage quality of potato tubers following exposure to gamma radiation from cobalt-60

Nucleonics 12, 16-7 (1954)

Sparrow, A. H. (See Jakowska, S.)

Sparrow, A. H., Pond, V. and Kojan, S.

Microsporogenesis in excised anthers of Trillium erectum grown on sterile culture media (A)

Records Genetics Soc.

Sparrow, A. H. (See Bowen, C. C.)

Sparrow, A. H. (See Sand, S. A.)

Sparrow, A. H

Stimulation and inhibition of plant growth by ionizing radiation (A)

Radiation Research J.

Sparrow, A. H. and Christensen, E.

Tolerance of certain higher plants to chronic exposure to gamma radiation from cobalt-60

Science 118, 697-8 (1953)

Sparrow, A. H. and Gunckel, J. E.

Tumor formation in hybrid Nicotiana glauca $\mathrm{x}$ langsdorfii exposed to chronic gamma irradiation from cobalt-60 (A) Proc. 8th Intl. Botan. Cong.

Sparrow, A. H. and Schairer, L. A.

Tumor formation in Nicotiana hybrids exposed to chronic gamma irradiation (A)

Plant Physiol.

Sparrow, A. H. (See Gunckel, J. E.)

Squicciarini, P. J.

An alcohol drip apparatus for the sliding microtome Lab. Investigations

Steele, R. (See Edelmann, A.)

Steele, $\mathbf{R}$.

The excretion and retention of the carbon of ingested sucrose by the mouse

J. Biol. Chem. 209, 91-102 (1954)

Steele, R. (See Grau, C. R.) 
Steffensen, D. and Arnason, T. J. Frequency of chromosome aberrations produced by fractional doses of $\mathrm{x}$-ray in Tradescantia Genetics 39, 220-8 (1954)

Stefferison, D.

Increased frequency of $\mathrm{x}$-ray-induced chromosomal aberrations in Tradescantia produced by a mineral-nutrient treatment (A)

Records Genetics Soc.

Steffensen, D.

Irregularities of chromosome divisions in Tradescantia grown on low sulfate

J. Expt1. Cell. Research 6, 554-6 (1954)

Stein, S. S. (See Koshland, D. E., Jr.)

Stein, S. S., and Koshland, D. E., Jr. Mechanism of hydrolysis of acetylcholine catalyzed by acetylcholinesterase and by hydroxide ion (L)

Arch. Biochem. and Biophys. 45, 468 (1953)

Sternheimer, E. P.

Method of culture and growth of maize endosperm in vitro Bull. Torrey Botan. Club 81, 111-3 (1954)

Sussman, A. S.

The effect of ionizing radiations upon the respiration and oxidases of the potato tuber.

J. Cellular and Comp. Physiol. 42, 273-83 (1953)

Sutton, E. (See Nims, L. F.)

Sutton, E. (See Scheraga, H. A.)

Taylor, J. H. (See Moses, M. J.)

Wilson, L. P. (See King, R. C.)

Wolin, H. (See Gibbs, M.)

Wood, E. M. (See King, R. C.)

Woodward, V. W.

Genetic and physiologic differences in neurospora induced by different physical mutations (A)

Radiation Research J.

Woodward, V. W.

Mutation rates at one glutamic acid locus in Neurospora crassa (A)

Genetics

\section{Instrumentation and Health Physics Department}

Axel, $P$

Escape peak correction to gamma ray intensity measurements made with sodium iodide crystals BNL 271 (T-44)

Bernstein, W. (See Weiss, J.)

Bernstein, W. (See Perlman, M. L.-Chemistry)

Bernstein, W. (See Finston, H. L.-Nuclear Engineering)

Bernstein, W. (See Weiss, M. M.)

Bernstein, W. (See Katcoff, S. Chemistry)

Bernstein, W., Weiss, J. and Kuper, J. B. H.

The oxidation yield of the ferrous sulfate dosimeter by $\mathrm{x}$-rays (A)

Bull. Am. Phys. Soc. 29, 21 (1954)

Bernstein, W'. (See Schwartz, R. B. Chemistry)

Chase, R. L.

Coincidence gray wedge pulse height analyzer BNL 263 (T-42)

Cowan, F. P. and Handloser, J. S.

Health physics program for the Brookhaven Cosmotron BNL 264 (T-43)

Cowan, F. P.

Isotope shipment and disposal

Chapter for Handbook of Radiation Dosimetry
Cowan, F. P.

Brookhaven National Laboratory neutron personnel monitoring service

*Ra-Det 6, 23-30 (1953)

Handloser, J. S. (See Cowan, F. P.)

Handloser, J. S. and Higinbotham, W. A.

A high sensitivity fast neutron survey meter Rev. Sci. Jnstr. 25, 98-9 (1954)

Higinbotham, W. A. (See Handloser, J. S.)

Kuper, J. B. H. (See Weiss, J.)

Kuper, J. B. H. (See Bernstein, W.)

Schardt, A. W. (See Mihelich, J. W.-Physics)

Schardt, A. W.

Gray wedge pulse height analysis. II

BNL 237 (T-37)

Schardt, A. W. (See Welker, J. P. Chemistry)

Weiss, J., Bernstein, W. and Kuper, J. B. H.

The absolute yield of the ferrous sulfate oxidation reaction J. Chem Phys. 22, 1593-6 (1954)

Weiss, J. (See Bernstein, W.)

Weiss, J. (See Manowitz, B.-Nuclear Engineering)

Weiss, J. (See Johnson, E. R. Chemistry)

Weiss, M. M. and Bernstein, W.

Degradation of gamma rays in water

Phys. Rev. 92, 1264 (1953)

Weiss, M. M.

*A study of the airborne radioactivity at Brookhaven $\mathrm{Na}$ tional Laboratory from the Nevada tests, March through June, 1953

BNL 252 (T-39)

Chemistry Depariment

Allen, A. O. (See Schwarz, H. A.)

Allen, A. O

Radiation damage to water $(A)$

Chem. Eng. Progr. (In press)

Allen, A. O. (See Saldick, J.)

Allen, A. O

The yields of free $\mathrm{H}$ and $\mathrm{OH}$ in the irradiation of water Radiation Research 1, 85-96 (1953)

Anderson, R. C., Ponder, E. and Ponder, R. V.

The combination between hymolysins and red cells or ghosts, as studied with a radioactive lysin and with new color reactions

J. Gen. Physiol. 37, 411-22 (1954)

Anderson, R. C. Sce Dawson, R. F.)

Baker, E. (See Friedlander, G.)

Baker, E. (See Hudis, J.)

Bigeleisen, J. and Wolfsberg, $M$.

Errata. Temperature independent factor in the relative rates of isotopic three center reactions J. Chem. Phys. 22, 1264 (1954)

Bigeleisen, J. and Wolfsberg, M.

Fractionation of the carbon isotopes in decarboxylation reactions. VI. Comparison of the intermolecular isotope effects of a pair of isotopic isomers J. Chem. Phys. 21, 2120-1 (1953)

Bigeleisen, J. (See Weston, R. E., Jr.)

Bigeleisen, J. and Kant, A.

Low temperature mobility of hydrogen in uranium hybride. Exchange equilibrium of deuterium between hydrogen and uranium hydride J. Am. Chem. Soc. (In press)

*For official use only 
Bigeleisen, J. and Wolfsberg, $M$.

Temperature independent factor in the relative rates of isotopic three center reactions

J. Chem. Phys. 21, 1972-4 (1953)

Brennan, H. (See Helmholz, L.)

Christman, D. R. (See Dawson, R. F.)

Corliss, L. M. (See Hastings, J. M.)

Corliss, L. M. (See Brockhouse, B. N.-Reactor)

Corliss, L. M., Hastings, J. M. and Goldman, J. E.

Neutron diffraction study of the anisotropy transition in $\mathrm{Fe}_{2} \mathrm{O}_{3}$ (L)

Phys. Rev. 93, 893-4 (1954)

Corliss, L. M. and Hastings, J. M.

A neutron diffraction study of zinc ferrite at low temperatures $(A)$

Intl. Union of Crystallography, Paris, July, 1954

Dawson, R. F., Christman, D. R. and Anderson, R. C. Alkaloid biogenesis. IV. The non-availability of nicotinic acid-carboxyl- $\mathrm{C}^{1.4}$ and its ethyl ester for nicotine biosynthesis. J. Am. Chem. Soc. 75, 5114-5 (1953)

Dawson, R. F. and Anderson, R. C.

Some general aspects of the use of isotopes in plant chemistry. $(A)$

Presented at Bowman-Gray School of Medicine, WinstonSalem, N. C., October, 1953

Dodson, R. W. (See Wolfgang, R. L.)

Dodson, R. W.

Salt and acid effects on the thallous-thallic exchange reaction J. Am. Chem. Soc. 75, $1795-7$ (1953)

Duffield, R. B. and Sugarman, N.

Disintegration of heavy elements by $2.2-\mathrm{Bev}$ protons (A)

Bull. Am. Phys. Soc. 29, 39 (1954)

Elliott, $N$.

Magnetic behavior of the system $\mathrm{CoO}-\mathrm{MgO}$ at elevated temperatures

J. Chem. Phys.

Elliott, N. (See Epstein, C.)

Epstein, C. and Elliott, N.

The magnetic susceptibilities of $\mathrm{K}_{3} \mathrm{MoCL}_{6}$ and $\mathrm{RuCL}_{3}$

J. Chem. Phys. 22, 634-5 (1954)

Fishman, L. M. and Harbottle, G.

The Szilard-Chalmers reaction in aqueous solutions of triand hexavalent chromium

J. Chem. Phys. 22, 1088-93 (1954)

Freed, S., Sancier, K. M. and Sporer, A. H.

A chlorophyll substance possessing a spectrum very similar to that of chlorphyll $b$

J. Am. Chem. Soc. (In press)

Freed, S. (See Sporer, A. H.)

Freed, S. and Sancier, K. M.

Photochemical activity of salts of uranium in solutions at the temperature of liquid nitrogen

J. Chem. Phys. 22, 928-9 (1954)

Freed, S. and Sancier, K. M.

Solvates of chlorophylls and related substances and their equilibria

J. Am. Chem. Soc. 76, 198-205 (1954)

Friedlander, G. (See Wolfgang, R. L.)

Friedlander, G. (See Welker, J. P.)

Friedlander, G., Miller, J. M., Wolfgang, R., Hudis, J. and

Baker, E.

Nuclear reactions of copper with 2.2-Bev protons (L)

Phys. Rev. 94, 727-8 (1954)

Friedlander, G. (See Hudis, J.)
Friedlander, G., Baker, E., Hudis, J., Miller, J. M. and Wolfgang, $R$.

Radioactive products from the interaction of $2.2-\mathrm{Bev}$ protons with copper $(\mathrm{A})$

Bull. Am. Phys. Soc. 29, 38 (1954)

Friedman, L. and Irsa, A. P.

Ruthenium isotope abundance $(\mathrm{N})$

J. Am. Chem. Soc. 75, 5741 (1953)

Goldman, J. E. (See Corliss, L. M.)

Gotimer, J. and Weinstein, A.

A simple chassis holder for use with relay racks (N) Nucleonics

Hamill, W. H. (See Williams, R. R., Jr.)

Hamill, W. H. and Williams, R. R., Jr.

On the scavenger effect in the $n, \gamma$ reaction in carbon tetrachloride

J. Chem. Phys. 22, 53 (1954)

Harbottle, G.

Activation of nuclear isomers by gamma radiation

Nucleonics 12, 64-7 (1954)

Harbottle, G.

The half-life of thallium-204

Phys. Rev. 91, 1234 (1953)

Harbottle, G. and Maddock, A. G

The preparation of chromium-51 of high specific activity J. Chem. Phys. 21, 1686-7 (1953)

Harbottle, G. (See Fishman, L. M.)

Harbottle, G.

The Szilard-Chalmers reaction in crystalline compounds of chromium

J. Chem. Phys. 22, 1083-8 (1954)

Hastings, J. M., Weiss, R. J., DeMarco, J. J., Weremchuk, G. and Corliss, L. M.

Anisotropic Debye-Waller factors in cubic crystals (A) Bull. Am. Phys. Soc. 29, 22 (1954)

Hastings, J. M. and Corliss, L. M. Crystal structure studies with neutrons (A) Am. Chem. Soc. Meeting, September, 1954

Hastings, J. M. (See Brockhouse, B. N.-Reactor)

Hastings, J. M. (See Corliss, L. M.)

Helmholz, L., Brennan, H. and Wolfsberg, $M$.

The spectra and electronic structure of fluorochromatic ion, chromyl chloride and some related compounds J. Chem. Phys.

Howland, J. J., Jr. (See Welker, J. P.)

Hudis, J. (See Friedlander, G.)

Hudis, J., Baker, E. and Friedlander, G Production of $\mathrm{Be}^{\top}$ in high energy reactions (A) Bull. Am. Phys. Soc. 29, 24 (1954)

Hudis, J., Wolfgang, R., Sugarman, N. and Friedlander, G. Reactions of aluminum with 2.2-Bev protons (A)

Bull. Am. Phys. Soc. 29, 38 (1954)

Irsa, A. P. (See Friedman, L.)

Johnson, E. R.

The production of iodine from solutions of potassium iodide irradiated with hard $\mathrm{x}$-rays (L)

J. Chem. Phys. 21, 1417 (1953)

Johnson, E. R. and Weiss, J.

The radical pair yield of ionizing radiation in sulfuric acid solutions J. Chem. Phys. 22, 752 (1954)

Kant, A.

Kinetics of complex isotopic exchange reactions J. Chem. Phys.

Kant, A. (See Bigeleisen, J.)

Katcoff, S. (See Markowitz, S. S.)

Katcoff, S. (See McKeown, M.-Physics) 
Katcoff, S. (Sec Bernstein, W.Instrumentation and Health Physics)

Katcoff, S. and Rubinson, W.

Yield of $\mathrm{Xe}^{1: 33}$ in the thermal neutron fission of $\mathrm{U}^{2: 55}$

Phys. Rev. 91, 1458-69 (1953)

Losee, J. P., Jr. (See Schwarz, H. A.)

Markowitz, S. S., Bernstein, W. and Katcoff, S.

A new 3.0-min Ce fission product and its 5.95-hr $\mathrm{Pr}$ daughter

Phys. Rev. 93, 178-80 (1954)

Markowitz, S. S. (See Bernstein, W.-Instrumentation and Health Physics)

Matlow, S. L.

An LCAO-MO study of the phenomenon thermochromism J. Chem. Phys.

Matlow, S. L.

An LCAO-MO study of the structure of chorophyll prototypes

J. Chem. Phys.

Miller, J. M. (See Zuber, A. V.)

Miller, J. M. (See Friedlander, G.)

Miskel, J. A. (See Perlman, M. L.)

Miskel, J. A. and Perlman, M. L.

Double vacancies in the $\mathrm{K}$-shell associated with $\mathrm{K}$-electron capture in $\mathrm{A}^{\text {37 }}$

Phys. Rev. 94, 1683-7 (1954)

Nielsen, E. and Weinstein, A. A technique for the production of thin sulfur films (N) Rev. Sci. Instr. 24, 1146 (1953)

Owen, H. R. (See Schaeffer, O. A.)

Owen, H. R. and Schaeffer, O. A.

The isotope abundances of chlorine from various sources (A)

ASTM Meeting, New Orleans, May, 1954

Perlman, M. L. and Miskel, J. A.

Average charge on the daughter atoms produced in the decay of $\mathrm{A}^{37}$ and $\mathrm{Xe}^{131 \mathrm{~m}}$

Phys. Rev. 91, 899-902 (1953)

Perlman, M. L., Bernstein, W. and Schwartz, R. B.

The decay scheme of $\mathrm{Ag}^{108}$

Phys. Rev. 92, 1236-40 (1953)

Perlman, M. L., and Welker, J. P.

The disintegration of $\mathrm{I}^{120}$

Phys. Rev. 95, 133 (1954)

Perlman, M. L. (See Miskel, J. A.)

Perlman, M. L. (See Schwartz, R. B.)

Rowland, F. S., Turton, C. N. and Wolfgang, R. L. A one-step method of synthesizing tritium-labeled compounds $(A)$

Am. Chem. Soc. Meeting, New York, September, 1954

Rubinson, W. (See Katcoff, S.)

Saldick, J.

Concentration effects on the solvent extraction and anion exchange of some metal halides (A)

Am. Chem. Soc. Meeting, New York, September, 1954

Saldick, J. and Allen, A. O.

The yreld of oxidation of ferrous sulfate in acid solution by high energy cathode rays

J. Chem. Phys. 22, 438-42 (1954)

Sancier, K. M. (See Sporer, A. H.)

Sancier, K. M. (See Freed, S.)

Schaeffer, O. A. and Owen, H. R.

Electron impact studies of isotopic oxygen and carbon dioxide molecules (A)

Bull. Am, Phys. Soc. 29, 27 (1954)
Schaeffer, O. A.

An improved mass spectrometer ion source

Rev. Sci. Instr. 25, 660-2 (1954)

Schaeffer, O. A. (See Owen, H. R.)

Schuler, R. H.

Chemical effects of $(d, p),(n, 2 n)$, and $(\gamma, n)$ activation of iodine

J. Chem. Phys. (In press)

Schuler, R. H.

The effect of iodine on the infrared spectra of the alkyl iodides (L)

J. Chem. Phys. 22, 947, (1954)

Schwartz, R. B. (See Perlman, M. L.)

Schwartz, R. B., Perlman, M. L. and Bernstein, W. Radiations from $\mathrm{Rb}^{\mathrm{*}}{ }^{\mathrm{i}}$ and $\mathrm{Rb}^{86 \mathrm{~m}}$

Phys. Rev. 91, 883-4 (1953)

Schwarz, H. A. and Allen, A. O

An economical water- and earth-shielded cobalt-60 gammaray source $(\mathrm{N})$

Nucleonics 12, 58-9 (1954)

Schwarz, H. A., Losee, J. P., Jr., and Allen, A. O

Hydrogen yields in the radiolysis of aqueous solutions J. Am. Chem. Soc. (In press)

Schwarz, H. A.

Temperature coefficient of the radiation-induced oxidation of ferrous sulfate $(\mathrm{N})$

J. Am. Chem. Soc. 76, 1587 (1954)

Slavin, $M$.

Photographic plate with constant contrast (L)

J. Opt. Soc. Am.

Sporer, A. H. (See Freed, S.)

Sporer, A. H., Freed, S. and Sancier, K. M.

Paper chromatography of chlorophylls

Science 119, 68-9 (1954)

Stam, P. B. and White, H. H., Jr.

The application of radiochemical techniques to the study of the interaction of hair fibers with aqueous solutions Textile Research J

Sugarman, N. (See Duffield, R. B.)

Sugarman, N. (See Turkevich, A.)

Sugarman, N. and Turkevich, A.

Production of gallium, germanium and arsenic nuclides by 2.2 Bev protons on copper (A)

Bull. Am. Phys. Soc. 29, 38 (1954)

Sugarman, N. (See Hudis, J.)

Taylor, T. I. (See Eisberg, R. M.)

Turkevich, A.

Estimation of beam intensity and cross sections at the Cosmotron from the gross radioactivity in copper (A) Bull. Am. Phys. Soc. 29, 38 (1954)

Turkevich, A. and Sugarman, N. Gallium, germanium and arsenic nuclides produced in the bombardment of copper with 2.2-Bev protons (L) Phys. Rev. 94, 728 (1954)

Turkevich, A. (See Sugarman, N.)

Wrahl, A. C. and Deck, C. F.

Rate of the ferrocyanide-ferricyanide exchange reaction (L) J. Am. Chem. Soc.

Weinstein, A. (See Gotimer, J.)

Weinstein, A. (See Nielsen, E.)

Weissman, S. I.

The fluorescence spectrum of triphenylmethyl at $4^{\circ} \mathrm{K}(\mathrm{L})$ J. Chem. Phys. 22, 155 (1954)

Welker, J. P. (See Perlman, M. L.)

Welker, J. P., Schardt, A. W., Friedlander, G. and Howland, J. J., Jr.

Metastable states of $\mathrm{Ge}^{\mathrm{i}: 3}$

Phys. Rev. 92, 401-5 (1953) 
Weston, R. E., Jr. and Bigeleisen, J

Kinetics of the exchange of hydrogen between phosphine and water: A kinetic estimate of the acid and base strengths of phosphine

J. Am. Chem. Soc. 76, 3074 (1954)

Weston, R. E., Jr.

Vibrational energy level splitting and optical isomerism in pyramidal molecules of the type $\mathrm{XY}_{3}$

J. Am. Chem. Soc. 76, 2645-8 (1954)

Weston, R. E., Jr.

The solubility of phosphine in aqueous solutions (N) J. Am. Chem. Soc. 76, 1027 (1954)

Williams, R. R., Jr. and Hamill, W. H

Chemical effects of electron capture by solutes in hydrocarbons during gamma irradiation

Radiation Research 1, 158-64 (1954)

Williams, R. R., Jr. (See Hamill, W. H.)

Wolfgang, R. L. and Friedlander, G.

Excitation function of the $\mathrm{C}^{12}$ ( $\left.\mathrm{p}, \mathrm{pn}\right) \mathrm{C}^{11}$ reaction in the Bev region

Phys. Rev. 96, 190-1 (1954)

Wolfgang, R. L. and Dodson, R. W.

Kinetics of exchange and disproportionation reactions in mercuric cyanide solutions

J. Am. Chem. Soc. 76, 2004-10 (1954)

Wolfgang, $R$. and Friedlander, $G$.

Momentum transfer in high energy nuclear interactions in aluminum $(A)$

Bull. Am. Phys. Soc. 29, 38 (1954)

Wolfgang, R. (See Friedlander, G.)

Wolfgang, R. L. (See Rowland, F. S.)

Wolfgang, R. L. (See Hudis, J.)

Wolfsberg, $M$.

Dipole velocity and dipole length matrix elements in $\pi$-electron systems and configuration interaction

J. Chem. Phys.

Wolfsberg, M. (See Bigeleisen, J.)

Wolfsberg, $\mathrm{M}$.

Note on a nitrogen molecule calculation

J. Chem. Phys. 21, 2166-9 (1953)

Wolfsberg, M. (See Helmholz, L.)

Zuber, A. V. and Miller, J. M

Chemical effects of neutron capture in some cobaltic hexammines (A)

Am. Chem. Soc. Meeting New York, September, 1954

\section{Cosmotron Department}

Adair, R. K.

Nuclear potential well depth (L)

Phys. Rev. 94, 737-8 (1954)

Adair, R. K.

Resonances in $\mathrm{Li}^{7}(\mathrm{p}, \mathrm{n}) \mathrm{Be}$

Phys. Rev.

Blewett, J. P., Blewett, M. H., Green, G. K., Moore, W. H and Smith, L. W

Magnet, Part I. Design

Rev. Sci. Instr. 24, 737-42 (1953)

Blewett, J. P., Blewett, M. H., Moore, W. H. and Smith, L. W. Pole-face windings, Part I. Design

Rev. Sci. Instr. 24, 773-6 (1953)

Blewett, J. P.

RF system, Part I. Design principles

Rev. Sci. Instr. 24, 779-81 (1953)

Blewett, J. P., Rogers, E. J. and Swartz, C. E.

RF system, Part II. Frequency control

Rev. Sci. Instr. 24, 782-8 (1953)
Blewett, J. P., Plotkin, M. and Rogers, E. J.

RF system, Part IV. Accelerating unit

Rev. Sci. Instr. 24, 795-800 (1953)

Blewett, J. P., Blewett, M. H. and Plotkin, M.

RF system, Part V. Properties of ferromagnetic ferrites Rev. Sci. Instr. 24, 800-5 (1953)

Blewett, $\mathrm{M}$. $\mathrm{H}$.

The Cosmotron. A review

Rev. Sci. Instr. 24, 725-37 (1953)

Blewett, M. H

Eddy current phenomena in the Cosmotron

Rev. Sci. Instr. 24, 856-60 (1953)

Blewett, M. H. (See Blewett. J. P.)

Blewett, M. H., Kelly, J. M. and Moore, W. H. Magnet, Part IV. Testing program for the individual blocks of the magnet

Rev. Sci. Instr. 24, 760-4 (1953)

Blewett, M. H., Green, G. K., Kassner, R. R., Moore, W. H., Smith, L. W. and Snyder, H. S.

Studies of injection phenomena in the Cosmotron

Rev. Sci. Instr. 24, 861-8 (1953)

Chen, F. F., Leavitt, C. P. and Shapiro, A. M.

Attenuation of $870-\mathrm{Mev}$ protons by various elements (A) Bull. Am. Phys. Soc. 29, 47 (1954)

Chen, F. F. (See Shapiro, A. M.)

Cottingham, J. G., Moore, W. H., Rogers, E. J. and Turner,

C. $\mathbf{M}$.

Injection system, Part II. Injection optics

Rev. Sci. Instr. 24, 816-20 (1953)

Courant, E. D. and Sternheimer, R. M.

Calculations of the protons' radial oscillations at injection Rev. Sci. Instr. 24, 868-9 (1953)

Courant, E. D.

Interaction of high energy pions with nuclei (L)

Phys. Rev. 94, 1081-2 (1954)

Courant, E. D.

Revision of gas scattering theory

Rev. Sci. Instr. 24, 836-7 (1953)

Dexter, E. W.

Control system, Part II. Timing system

Rev. Sci. Instr. 24, 846-7 (1953)

Giordano, S., Green, G. K. and Rogers, E. Control system, Part III. Peaking strips Rev. Sci. Instr. 24, 848-50 (1953)

Green, G. K. and Kassner, R. R

Control system, Part I. Central control and wiring Rev. Sci. Instr. 24, 838-45 (1953)

Green, G. K. (See Giordano, S.)

Green, G. K. (See Blewett, J. P.)

Green, G. K., Kassner, R. R., Moore, W. H. and Smith, L. W Magnet, Part II. Magnetic measurements

Rev. Sci. Instr. 24, 743-54 (1953)

Green, G. K. and Shelton, E. E. Magnet, Part VI. Power supply Rev. Sci. Instr. 24, 769-72 (1953)

Green, G. K. (See Blewett. M. H.)

Hill, D. A., Coor, T., Hornyak, W. F., Smith, L. W. and Snow, G. A.

Experiment with neutrons from the Cosmotron.I (A) Bull. Am. Phys. Soc. 29, 54 (1954)

Hill, D. A. (See Snow, G. A.-Physics)

Jacobus, D. D. and Polk, 1. J.

Vacuum system, Part I. Vacuum chamber Rev. Sci. Instr. 24, 821-31 (1953)

Kassner, D. and Lasky, C

Vacuum system, Part II. Pumping system

Rev. Sci. Instr. 24, 832.5 (1953) 
Kassner, R. R. (See Green, G. K.)

Kassner, R. R. (See Blewett, M. H.)

Kelly, J. M. (See Blewett, M. H.)

Kosh, J. A. and Livingston, M. S.

Magnet, Part V. Coil

Rev. Sci. Instr. 24, 765-8 (1953)

Lasky, C. (See Kassner, D.)

Leavitt, C. P. (Sec Chen, F. F.)

Leavitt, C. P. (See Shapiro, A. M.)

Livingston, M. S. (See Kosh, J. A.)

Mede, J. J.

Pole-face windings, Part II. Fabrication

Rev. Sci. Instr. 24, 777-8 (1953)

Meyer, R. A. and Moore, W. H.

Magnet, Part III. Mechanical design, fabrication and erection

Rev. Sci. Instr. 24, 755-9 (1953)

Mills, R. (See Yang, C. N.)

Moore, W. H. (See Cottingham, J. G.)

Moore, W. H. (See Blewett, J. P.)

Moore, W. H. (See Green, G. K.)

Moore, W. H. (See Meyer, R. A.)

Moore. W. H. (See Blewett, M. H.)

Plotkin, M. and Rogers, E. J.

Generating if power for 3:Bev accelerator

Electronics, February, 1954

Plotkin, M. and Yuan, L. C. L.

Rf system, Part III. Power amplifier

Rev. Sci. Instr. 24, 789-94 (1953)

Plotkin, M. (See Blewett, J. P.)

Polk, I. J. (See Jacobus, D. D.)

Rogers, E. J. (See Giordano, S.)

Roğers, E. J. (See Plotkin, M.)

Rogers, E. J. and Turner, C. M

Injection system, Part I. Van de Graaff accelerator

Rev. Sci. Instr. 24, 805-15 (1953)

Rogers, E. J. (See Cottingham, J. G.)

Rogers, E. J. (See Blewett, J. P.)

Ross, M. H

A connection between pion photoproduction and scattering phase shifts

Phys. Rev. 94, 454 (1954)

Ross, M. H.

Meson-meson effects in meson nucleon scattering (A) Bull. Am. Phys. Soc. 29, 31, (1954)

Shapiro, A. M. (See Chen, F. F.)

Shapiro, A. M., Leavitt, C. P. and Chen, F. F. The total p-p cross section above $400 \mathrm{Mev}$ (A) Bull. Am. Phys. Soc. 29, 75 (1954)

Shapiro, A. M., Leavitt, C. P. and Chen, C. F The total $\pi^{-}$-p cross section at 840 and $470 \mathrm{Mev}(\mathrm{L})$ Phys. Rev. 92, 1073 (1953)

Shelton, E. E. (See Green, G. K.)

Smith, L. W. (See Hill, D. A.)

Smith, L. W. (See Snow, G. A.)

Smith, L. W. (See Blewett, J. P.)

Smith, L. W. (See Green, G. K.)

Smith, L. W. (See Blewett, M. H.)

Sternheimer, R. M. (See Courant, E. D.)

Sternheimer, R. M.

The density effect for the ionization loss at low energies Phys. Rev, 93, 351 (1954)

Sternheimer, R. M.

Double focusing of charged particles with a wedge-shaped, non-uniform magnetic field

Rev. Sci. Instr. 24, 573-85 (1953)
Sternheimer, R. M.

Effect of the atomic core on the nuclear quadrupole coupling

Phys. Rev. 95, 736-50 (1954)

Sternheimer, R. M.

Electronic polarizabilities of ions from Hartree-Fock wave functions $(A)$

Bull. Am. Phys. Soc. 29, 65 (1954)

Sternheimer, R. M

Kinematic criterion for meson production in fundamental particle collisions (L)

Phys. Rev. 93, 642 (1954)

Sternheimer, R. M. and Foley, H. M.

The nuclear quadrupole coupling in the $\mathrm{Li}_{2}$ molecule

Phys. Rev. 92, 1460-8 (1953)

Sternheimer, R. M., Foley, H. M. and Tycko, D.

The nuclear quadrupole coupling in polar molecules

Phys. Rev. 93, 734-42 (1954)

Sternheimer, R. M

Polarization of nucleons elastically scattered from nuclei (L)

Phys. Rev. 95, 587 (1954)

Sternheimer, R. M. (Snow, G. A.-Physics)

Stoughton, L. D.

The Cosmotron building

Rev. Sci. Instr. 24, 854-5 (1953)

Swartz, C. E.

Control system, Part IV. Pick-up electrodes

Rev. Sci. Instr, 24, 851-3 (1953)

Swartz, C. E. (See Blewett, J. P.)

Turner, C. M. (See Rogers, E. J.)

Turner, C. M. (See Cottingham, J. G.)

Turner, C. M.

Ionization loading of electrostatic generators (A)

Bull. Am. Phys. Soc. 29, 11 (1954)

Yang, C. N. and Mills, R.

Isotopic spin conservation and a generalized gauge invariance $(A)$

Bull. Am. Phys. Soc. 29, 43 (1954)

Yang, C. N. (See Snow, G. A. - Physics)

Yuan, L. C. L. and Lindenbaum, S. J.

The energy spectrum of negative pions produced in beryllium by $2.3-\mathrm{Bev}$ protons (L) Phys. Rev. 93, 1431 (1954)

Yuan, L. C. L. and Lindenbaum, S. J

The interaction cross section of hydrogen for $340-\mathrm{Mev}$ positive pions ( $\mathrm{L}$ ) Phys. Rev. (In press)

Yuan, L. C. L. (See Lindenbaum, S. J.-Physics)

Yuan, L. C. L. (See Plotkin, M.)

Medical Department

Ciereszko, L. S. and Hankes, L. V.

Intermediates in the synthesis of carboxyl $\mathrm{C}^{14}$ - labeled 3 hydroxyanthranilic acid

J. Am. Chem. Soc. 76, 2500 (1954)

Conn, H. L., Jr.

Accuracy of an isotope dilution (Stewart principle) method using radiopotassium in the measurement of cardiac output in the intact subject, as determined by a comparision of results with those obtained by the Fick procedure J. Clin. Invest

Conn, H. L., Jr. (See Stroud, R. C.)

Cotzias, G. C. (See Maynard, L. S.)

Cotzias, G. C., Serlin, I. and Greenough, J. I

"Insoluble" enzymes and the solubilization of monoamide oxidase (A)

Federation Proc. 13, No. 1, (1954) 
Cotzias, G. C. (See Dahl, L. K.)

Cotzias, G. C., Serlin, I. and Greenough, J. J.

Preparation of soluble monoamine oxidase Science 120, 144 (1954)

Dahl, L. K. and Love, R. A. Evidence for a relationship between sodium (chloride) intake and human essential hypertension J. Am. Med. Assoc. (In press)

Dahl, L. K., Stall, B. G., III, and Cotzias, G. C. Metabolic effects of marked sodium restriction in hypertensive patients: Changes in total exchangeable sodium and potassium J. Clin. Invest. (In press)

Dahl, L. K., Stall, B. G., III, and Cotzias, G. S Metabolic effects of marked sodium restriction in hypertensive patients. II. Evidence for a variable sodium pool with constant dietary intake J. Clin. Invest. (In press)

Dahl, L. K. and Love, R. A.

The relation of sodium chloride intake to essential hypertension in humans (A)

Federation Proc. 13, No. 1 (1954)

Drew, R. M

The effect of gamma radiation on the viability of Diplococcus pneumoniae. Type III (A) Bacteriol. Proc.

Drew, R. M.

Failure to demonstrate a relationship between tyrosine and the toxicity of diphtherial culture filtrates $(\mathrm{N})$ J. Bacteriol. 66, No. 2 (1953)

Dudley, H. C.

Pharmacological studies of radiogermanium $\left(\mathrm{Ge}^{71}\right)$ II Arch. Ind. Hyg. and Occupational Med. 8, 528-30 (1953)

Dudley, H. C.

Titanium phosphate $\left(\mathrm{P}^{32}\right)$ preparation as a means of delivering interstitial radiation $(A)$

Am. Chem. Soc. Meeting, Chicago, September, 1953

Dunham, E. T. (See Tosteson, D. C.)

Ebert, J. D.

The effects of chorio-allantoic transplants of adult chicken tissues on homologous tissues of the host embryo Proc. Natl. Acad. Sci. 40, 337-47 (1954)

Farr, L. E., Sweet, W. H., Robertson, J. S., Foster, C. G., Locksley, H. B., Sutherland, D. L., Mendelsohn, M. L. and Stickley, E. E.

Neutron capture therapy with boron in the treatment of glioblastoma multiforme

Am. J. Roentgenol. Radium Therapy Nuclear Med. 71, 279-93 (1954)

Farr, L. E., Robertson, J. S. and Stickley, E. E. Physics and physiology of neutron capture therapy (A) Natl. Acad. Sci. Meeting, Washington, April, 1954

Foster, C. G. (See Farr, L. E.)

Gamble, J. L., Jr.

Effect of variations of sodium intake and of desoxycorticosterone on the relationship of the sodium space to the chloride space in dogs

Am. J. Physiol. 175, 276-82 (1953)

Godwin, J. T., Foote, F. W., Jr., and Frazell, E. L. Acinic cell adenocarcinoma of parotid: report of 27 cases Am. J. Pathol. 30, 465-78 (1954)

Godwin, J. T. (See Robertson, J. S.)

Godwin, J. T. (See Stoner, R. D.)

Godwin, J. T. (See Shellabarger, C. J.)

Godwin, J. T. and Shellabarger, C. J. Studies on the thyroidal uptake of astatine in the rat J. Clin. Endocrinol. and Metabolism
Greenough, J. J. (See Cotzias, G. C.)

Hale, W. M. and Stoner, R. D.

Effects of ionizing radiation on immunity

Radiation Research (In press)

Hale, W. M. (See Stoner, R. D.)

Hankes, L. V. An electrical heater for the Van Slyke-Folch carbon combustion apparatus (N) Anal. Chem.

Hankes, L. V. (See Ciereszko, L. S.)

Hankes, L. V. (See Lutwak, L.)

Locksley, H. B. (See Farr, L. E.)

Love, R. A. (See Dahl, L. K.)

Lutwak, L., Hankes, L. V. and Van Slyke, D. D. A study of the alkaline-ash procedure for the estimation of serum protein bound iodine Anal. Chem.

Maynard, L. S. and Cotzias, G. C. The distribution of radiomanganese $\left(\mathrm{Mn}^{56}\right)$ in the tissues and cells of the rat (A)

Federation Proc. 13, No. 1 (1954)

Mendelsohn, M. L. (See Farr, L. E.)

Robertson, J. S. and Godwin, J. T.

Calculation of $\mathrm{I}^{1: 11}$ beta radiation dose to the bone marrow Brit. J. Radiol. 27, 241-2 (1954)

Robertson, J. S. (See Farr, L. E.)

Robertson, J. S. (See Tosteson, D. C.)

Serlin, I. (See Cotzias, G. C.)

Shellabarger, C. J.

The detection of thyroid stimulating hormone by $\mathrm{I}^{131}$ uptake in chicks

Am. J. Physiol. 6, 721 (1954)

Shellabarger, C. J.

Effects of cortisone on thyroid function in white leghorn cockerels

Endocrinology 55, 100 (1954)

Shellabarger, C. J. and Godwin, J. T.

The effects of thyroxine or triiodothyronine on the chick thyroid in the presence or absence of TSH

Am. J. Physiol. 176, 371-3 (1954)

Shellabarger, C. J. and Godwin, J. T.

Effects of triiodothyronine on tadpoles

Endocrinology 54, 230-2 (1954)

Shellabarger, C. J. and Godwin, J. T.

Studies on the thyroidal uptake of astatine in the rat J. Clin. Endocrinol. and Metabolism (In press)

Sinex, F. M. (See Sacks, J. - Biology)

Sinex, F. M. and Van Slyke, D. D.

The source of the hydroxylysine of collagen (A)

Federation Proc. 13, 297 (1954)

Stall, B. G., III (See Dahl, L. K.)

Stickley, E. E. (See Farr, L. E.)

Stickley, E. E.

Neutron capture therapy: Slow neutron depth dose measurements in tissues

Am. J. Roentgenol. Radium Therapy Nuclear Med.

Stickley, E. E., Pittinger, C. B., Featherstone, R. M., Gross, E. G. and Levy $L$.

Xenon concentration changes in brain and other body tissues of the dog during inhalation of the gas

J. Pharmacol. Exptl. Therap. 110, 458-62 (1954)

Stoner, R. D. and Godwin, J. T.

The effects of ACTH and cortisone upon acquired immunity to trichinosis in mice

Am. J. Pathol. 
Stoner, R. D. and Godwin, J. T.

The effects of ACTH and cortisone upon susceptibility to trichinosis in mice

Am. J. Pathol. 29, 943-50 (1953)

Stoner, R. D. (See Hale, W. M.)

Stoner, R. D. and Hale, W. M.

Increased susceptibility of mice to anaphylactic shock

following cobalt- 60 gamma radiation

J. Immunol. 72, 419-23 (1954)

Stoner, R. D. and Hale, W. M.

A method for eradication of the mite, Myocoptes musculinus, from laboratory mice

J. Econ. Entomol. 46, 692-3 (1953)

Stroud, R. C. and Conn, H. L., Jr. Pulmonary vascular effects of moderate and severe hypoxia in the dog

J. Appl. Physiol.

Sutherland, D. L. (See Farr, L. E.)

Tosteson, D. C. and Dunham, E. T.

The effect of sickling on sodium and cesium transport (A)

Federation Proc. 13, 523 (1954)

Tosteson, D. C. and Robertson, J. S

Potassium exchange in the red blood cells of the duck (A) Federation Proc. 12, 145 (1953)

Van Slyke, D. D. (See Sinex, F. M.)

Van Slyke, D. D. (See Lutwak, L.)

Van Slyke, D. D.

The wet carbon combustion and some of its applications Anal. Chem. (In press)

\section{Reactor Department}

Binns, J. E.

Electronic control of a nuclear reactor

Electronics 26, 130-1 (1953)

Borst, L. B. (See Kunstadter, J. W.)

Brockhouse, B. N.

Antiferromagnetism in cupric oxide (A)

Bull. Am. Phys. Soc. 29, 44 (1954)

Brockhouse, B. N., Corliss, L. M. and Hastings, J. M. Diffuse magnetic scattering of neutrons: Zinc ferrite (A)

Bull. Am. Phys. Soc. 28, 19 (1953)

Christensen, R. L.

Slow neutron resonances in tantalum

Phys. Rev. 92, 1509-10 (1953)

Floyd, J. J. (See Kunstadter, J. W.)

Foote, H. L., Jr. (See Sailor, V. L.)

Foote, H. L., Jr., Landon, H. H. and Sailor, V. L.

Slow neutron resonances in holmium, thulium, and lutecium Phys. Rev. 92, 656-9 (1953)

Foote, H. L., Jr.

Tellurium resonance scattering $(A)$

Bull. Am. Phys. Soc. 29, 53 (1954)

Kunstadter, J. W., Floyd, J. J. and Borst, L. B.

Long-lived delayed neutrons from fission Phys. Rev. 91, 594-6 (1955)

Landon, $\mathrm{H} . \mathrm{H}$.

Doppler effect in the slow neutron resonance in rhodium Phys. Rev, 94, 1215-17 (1954)

Landon, H. H. and Sailor, V. L. Slow neutron resonances in cesium, palladium and gold Phys. Rev. 93, 1030-33 (1954)

Landon, H. H. (See Sailor, V. L.)

Landon, H. H. (See Foote, H. L., Jr.)

McCormick, R. A. and Singer, I. A.

Power spectra of turbulent flow at 300 feet during unstable conditions at Brookhaven National Laboratory (A) Am. Meteor. Soc. Meeting, January, 1954
Powell, R. W.

Some general problems in operating a research reactor Nucleonics 11, 26-31 (1953)

Reier, M. and Shamos, M. H. Neutron capture gamma ray spectra (A) Bull. Am. Phys. Soc. 29, 48 (1954)

Sailor, V. L.

The parameters for the slow neutron resonance in rhodium Phys. Rev. 91, 53-7 (1953)

Sailor, V. L. (See Landon, H. H.)

Sailor, V. L., Landon, H. H. and Foote, H. L., Jr. Slow neutron resonances in europium Phys. Rev. 93, 1292-6 (1954)

Sailor, V. L. (See Foote, H. L., Jr.)

Singer, I. A. (See Smith, M. E.)

Singer, I. A.

A comparison of computed and measured ground-level dose rates from radioargon emitted by the Brookhaven reactor stack BNL 292 (T-49)

Singer, I. A. (See McCormick, R. A.)

Singer, I. A. and Simith, M. E.

Relation of gustiness to other meteorological parameters J. Meteor. 10, 121-6 (1953)

Singer, I. A. and Smith, M. E.

The use of averages in air pollution meteorology

Air Repair, February, 1954

Smith, M. E. and Singer, I. A. Applicability and key to meteorological punch card data, April 1950 - March 1952 BNL 288 (T-48)

Smith, M. E. (See Singer, I. A.)

Wood, R. E.

Activation measurements in europium with monoenergetic neutrons (A)

Bull. Am. Phys. Soc, 29, 57 (1954)

Wood, R. E.

Europium activation studies with monochromatic neutrons Phys. Rev. 95, 453-5 (1954)

Wood, R. E

Resonance scattering of $\mathrm{Ag}$ and $\mathrm{Au}$ (A)

Bull. Am. Phys. Soc. 29, 56 (1954)

Nuclear Engineering Department

Atherton, J. E., Gurinsky, D. H., Kammerer, O. F., Klamut,

C., Silberberg, M., Turovlin, B. and Weeks, J.

Studies in the uranium-bismuth (UBi) fuel system Nucleonics 12, No. 7 (1954)

Austin, J. C.

Floor inspector $(\mathrm{N})$

Nucleonics

Austin, J. C.

Mechanical shoe sander $(\mathrm{N})$

Nucleonics 12, 64-5 (1954)

Austin, J. C. and Richards, P.

Radiography as a hot laboratory service Nucleonics

Ballantine, D. S., Dienes, G. J., Manowitz, B., Ander, P. and

Mesrobian, R. B. Evaluation of polyethylene crosslinked by ionizing radiation $(\mathrm{L})$ J. Polymer Sci. 13, 410-4 (1954)

Ballantine, D. S., Colombo, P., Glines, A. and Manowitz, B. Gamma-ray-initiated polymerization of styrene and methyl methacrylate Nuc.Eng.

Ballantine, D. S. (See Dienes, G. J. - Physics) 
Ballantine, D. S

Progress report on fission products utilization. VI. Further studies of the effect of gamma radiation on vinyl polymer systems

BNL 294 (T-50)

Banks, H. O., Jr. (See Stang, L. G., Jr.)

Bareis, D. W. (See Wiswall, R. H., Jr.)

Bretton, R. H. (See Manowitz, B.)

Bryner, J. S. (See Teitel, R. J.)

Colombo, P. (See Ballantine, D. S.)

Doering, R. (See Stang, L. G., Jr.)

Dwyer, O. E.

Heat exchange in a liquid-metal-fuel reactor for power Nucleonics 12, No. 7 (1954)

Dwyer, O. E., Schomer, R. T., Sheehan, T. V., Weisman, J. and

Horn, F. L.

Heat transfer rates for cross-flow of water through a tube bank at Reynolds numbers up to a million 1953 Conf. on Nuc. Eng., Berkeley

Dwyer, O. E., Sheehan, T. V. and Schomer, R. T.

Heat transfer rates for cross-flow of water through a tube bank at Reynolds numbers up to a million. Part I. Average film coefficients for individual tubes Trans. Am. Soc. Mech. Eng.

Dwyer, O. E., Shechan, T. V. and Weisman, J.

Heat transfer rates for cross-flow of water through a tube bank at Reynolds numbers up to a million. Part II. Circumferential variation of film coefficient for individual tubes Trans. Am. Soc. Mech. Eng.

Dwyer, O. E., Horn, F. L. and Weisman, J.

Heat transfer rates for cross-flow of water through a tube bank at Reynolds numbers up to a million. Part III. Forced convection boiling and pressure drop data

Trans. Am. Soc. Mech. Eng.

Faucher, J. A. and Thomas, H. C. Adsorption studies on clay minerals. IV. The system montmorillonite-cesium-potassium J. Chem. Phys. 22, 258 (1954)

Finston, H. L. and Bernstein, W. The decay scheme of $\mathbf{I}^{132}$

Phys. Rev. 96, 71-4 (1954)

Fleck, J. A.

The energy-dependent Boltzmann equation applied to criticality calculations for bare graphite-moderated reactors BNL 298 (T-51)

Ginell, W. S. (See Hatch, L. P.)

Glines, A. (See Ballantine, D. S.)

Gurinsky, D. H. (See Teitel, R. J.)

Gurinsky, D. H. (See Atherton, J. E.)

Hatch, L. P. (See Miles, F. T.)

Hatch, L. P. (See Manowitz, B.)

Hatch, L. P., Martin, J. J. and Ginell, W. S. Ultimate disposal of radioactive wastes Nucleonics (In press)

Heus, R. J. (See Miles, F. T.)

Horn, F. L. (See Dwyer, O. E.)

Horn, F. L.

Slug charging interlock

Nucleonics

Horrigan, R. V. (See Manowitz, B.)

Huszagh, D. W.

Remote handling mechanisms employed in the permanent waste disposal pilot plant

Nucleonics

Kammerer, O. F. (See Atherton, J. E.)

Kaplan, I. (See Miles, F. T.)

Klamut, C. (See Atherton, J. E.)
Kuhl, O. A., Rosenzweig, W. and Manowitz, B. Curie evaluation of hollow cylinder sources BNL 260 (T-41)

Manowitz, B. (See Kuhl, O. A.)

Manowitz, B. (See Ballantine, D. S.)

Manowitz, B.

The industrial future of radiation chemistry Nucleonics 11, 18-20 (1954)

Manowitz, B., Bretton, R. H. and Horrigan, R. V.

The occurrence and control of radioactive entrainment in evaporative systems Chem. Eng. Prog.

Manowitz, B. and Hatch, L. P. Processes for high level waste disposal Nuc. Eng. (In press)

Manowitz, $\mathrm{B}$.

The use of the ionization power of nuclear reactors for profitable chemical processes

Nuc. Eng. (In press)

Manowitz, B., Horrigan, R. V., Weiss, J., Tarpley, W. and

Yudis, $\mathbf{M}$.

Radiation sterilization: Il. The effect of high energy gamma radiation from kilocurie radioactive sources on steroid hormones Indust. Eng. Chem. 46, 1458-64 (1954)

Manowitz, B., Richards, P. and Horrigan, R. V.

Vapor compression evaporation of Brookhaven's radioactive liquid wastes

Am. Inst. Chem. Eng. Regional Meeting, Cleveland, Ohio

Martin, J. J. (See Hatch, L. P.)

Miles, F. T., Wiswall, R. H., Heus, R. J. and Hatch, L. P. A continuously separating breeder blanket using thorium fluoride

Nucleonics 12, No. 7 (1954)

Miles, F, T. and Williams, $C$.

Liquid metal fuel reactor systems for power Nucleonics 12, No. 7 (1954)

Miles, F. T. and Kaplan, I. Optimizing and comparing reactor designs Nuc. Eng. (In press)

Mills, T. H. (See Stang, L. G., Jr.)

Oleksa, $\mathrm{S}$.

The inelastic scattering cross section of lead BNL 273 (T-45)

Rand, A. C. (See Stang, L. G., Jr.)

Raseman, C. J. and Weisman, J.

Liquid metal fuel reactor processing loops

Nucleonics 12, No. 7 (1954)

Richards, P. (See Austin, J. C.)

Richards, P. (See Manowitz, B.)

Rosenzweig, W. (See Kuhl, O. A.)

Schomer, R. T. (See Dwyer, O. E.)

Selvin, G. (See Stang, L. G., Jr.)

Sheehan, T. V. (See Dwyer, O. E.)

Silberberg, M. (See Atherton, J. E.)

Stang, L. G., Jr., Strickland, G., Rand, A. C. and Selvin, G. Design and performance of a portable irradiation unit (N) Nucleonics 12, 62-3 (1954)

Stang, L.G., Jr., Tucker, W. D., Banks, H. O., Jr., Doering, R. and Mills, T. H.

Production of iodine-132

Nucleonics 12, 22 (1954)

Strickland, G. (See Stang, L. G., Jr.)

Susskind, $\mathrm{H}$.

A low cost heater for welding plastic sheets

Nucleonics 
Teitel, R. J., Gurinsky, D. H. and Bryner, J. S. Liquid metal fuels and liquid metal breeder blankets Nucleonics 12, No. 7 (1954)

Tucker, W. D. (See Stang, L. G., Jr.)

Thomas, H. C. (See Faucher, J. A.)

Turovlin, B. (See Atherton, J. E.)

Weeks, J. (See Atherton, J. E.)

Weeks, J. (See Atherton, J. E.)

Weisman, J. (See Dwyer, O. E.)

Weisman, J. (See Raseman, C. J.)

Williams, C. (See Miles, F. T.)

Winsche, W. E. (See Wiswall, R. H., Jr.)

Wiswall, R. H. (See Miles, F. T.)

Wiswall, R. H., Jr., Bareis, D. W. and Winsche, W. E. The processing of liquid bismuth alloys by fused salts Nucleonics 12, No. 7 (1954)

\section{Photography and Duplicating Division}

Smith, R. F. (See Saunders, L. Z. - Biology)

Smith, R. F.

The use of polarized light in the photography of unstained histological radioautographs of plant tissues J. Biol. Photo. Assoc. 22. 15 (1954)

\section{Physics Department}

Alburger, D. E.

Energy levels in $\mathrm{Pb}^{206}$

Article for book Beta and Gamma Ray Spectroscopy

Alburger, D. E. and Pryce, M. H. L.

Energy levels in $\mathrm{Pb}^{206}$ from the decay of $\mathrm{Bi}^{206}$

Phys. Rev. 95, 1482-99 (1954)

Alburger, D. E.

Gamma radiation from charged particle reactions

Article for book Beta and Gamma Ray Spectroscopy

Alburger, D. E.

Gamma-ray spectroscopy with intermediate-image focusing Rev. Sci. Instr.

Augustyniak, W. (See McReynolds, A. W.)

Baker, C. P. (See Draper, J. E.)

Blau, M. and Caulton, M.

Inelastic scattering of $500-\mathrm{Mev}$ negative pions (A)

Am. Phys. Soc. Meeting, Washington

Blau, M. (See Caulton, M.)

Blau, M., Caulton, M. and Smith, J. E. Meson production by $500-\mathrm{Mev}$ negative pions (L) Phys. Rev. 92, 516 (1953)

Blau, M., Oliver, A. R. and Smith, J. E. Neutron and meson stars induced in the light elements of the emulsion

Phys. Rev. 91, 949-57 (1953)

Carter, R. S. (See Palevsky, H.)

Carter, R. S. (See Pilcher, V. E.)

Carter, R. S. and Harvey, J. A.

Parameters of neutron resonances in $\mathrm{Cs}, \mathrm{In}, \mathrm{Sn}, \operatorname{Pr}$ (A) Bull. Am. Phys. Soc. 29, 57 (1954)

Carter, R. S., Palevsky, H., Myers, V. W. and Hughes, D. J. Thermal absorption cross sections of boron and gold Phys. Rev. 92, 716-21 (1953)

Caulton, M. (See Blau, M.)

Caulton, M., Blau, M. and Smith, J. E. Interactions of $500-\mathrm{Mev}$ negative pions with emulsion nuclei (A) Bull. Am. Phys. Soc. 28, 15 (1953)

Church, E. L. and Goldhaber, M. Low-energy transitions in gadolinium and related rare-earth activities (A)

Bull. Am. Phys. Soc. 29, 38 (1954)
Cohen, V. W., Corngold, N. R. and Ramsey, N. F. Experiment for measurement of the magnetic moment of the neutron $(A)$ Bull. Am. Phys. Soc. 28, 7 (1953)

Cohen, V.W., Burke, B. F., Strandberg, M.W.P. and Koski, W. The nuclear magnetic moment of $S^{35}$ by microwave spectroscopy Phys. Rev. 93, 193-4 (1954)

Cohen, V. W., Hardy, W. A., Silvey, G., Townes, C. H.,

Burke, B. F., Strandberg, M. W. P. and Parker, G. W. The nuclear moments of $\mathrm{Se}^{7 \%}$ Phys. Rev. 92, 1532-7 (1953)

Cohen, V. W. and Gilbert, D. A

The nuclear spin and hyperfine structure interaction of the 3-hr $\mathrm{Cs}^{1.44}$ isomer (L)

Phys. Rev. 95', 569 (1954)

Cohen, V. W. (See Kikuchi, C.)

Cool, R. L., Madansky, L. F. and Piccioni, O. Total cross section of pions at 1.5 Bev (L) Phys. Rev. 93, 249 (1954)

Cool, R. L., Madansky, L. and Piccioni, O. Total interaction cross section of negative pions at kinetic energies of 1.0 and $1.45 \mathrm{Bev}(A)$ Bull. Am. Phys. Soc. 28, 14 (1953)

Cool, R. L., Madansky, L. and Piccioni, O. Total interaction cross section of pions with protons and deuterons at $1.0 \mathrm{Bev}(\mathrm{L})$

Phys. Rev. 93, 637 (1954)

Coor, T. (See Hornyak, W. F.)

Coor, T. (See Hill, D. A.Cosmotron)

Coor, T. (See Snow, G. A.)

Corngold, N. R. (See Cohen, V. W.)

der Mateosian, E. and Wu, C. S.

The $\beta$-spectrum of $\mathrm{I}^{129}$ and its decay scheme

Phys. Rev. 95, 458 (1954)

der Mateosian, E. (See Muehlhause, C. O.)

der Mateosian, E.

Further applications of the "internal source" scintillation spectrometer (A)

Bull. Am. Phys. Soc, 29, 58 (1954)

der Mateosian, E.

Scintillation spectrometer determination of $\mathbf{L}$ capture to $\mathrm{K}$ capture ratio in $\mathrm{Cd}^{109}$ and $\mathrm{I}^{125}$

Phys. Rev. 92, 938-40 (1953)

de Shalit, A. and Goldhaber, M. Mixed configurations in nuclei Phys. Rev. 92, 1211-18 (1953)

de Shalit, A. (See Mihelich, J. W.)

Dienes, G. J. and Levy, P. W. Color centers produced by reactor neutron irradiation (A) Bristol Conf. on Defects in Crystalline Solids, Bristol, England, July 13-17, 1954.

Dienes, G. J. (See Ballantine, D. S.-Nuclear Engineering)

Dienes, G. J. Mesrobian, R. B., Ander, P. and Ballantine, D. S. Gamma-ray polymerization of acrylamide in the solid state (L) J. Chem. Phys. 22, 565 (1954)

Dienes, G. J. (See Levy, P. W.)

Dienes, G. J. Mechanical properties and imperfections in crystals Chapter for book Treatise in Rheology

Dienes, G. J. Mechanical properties and lattice imperfections (A) Rheology Soc. Annual Meeting

Dienes, G. J. (See Vineyard, G. H.)

Dienes, G. J. (See Rosenblatt, D. B.) 
Dienes, G. J.

Variable activation energy and the motion of lattice defects (L) Phys. Rev. 91, 1283 (1953)

Dienes, G. J., Chipman, D. L. and Warren, B. E.

$\mathrm{X}$-ray measurements of radiation damage in black phosphorus (L)

J. Appl. Phys. 24, 1251 (1953)

Draper, J. E. and Baker, C. P.

Self-indication measurement of $\sigma_{\mathrm{to}}$ for the 5.2-ev neutron reasonance in silver $(A)$

Bull. Am. Phys. Soc. 29, 56 (1954)

Eisberg, R. M.

Energy spectra of nucleons evaporated from a compound nucleus ( $L$ )

Phys. Rev. 94, 739-40 (1954)

Eisberg, R. M. (See Palevsky, H.)

Eisberg, R. M., Palevsky, H., Rickey, M. W. and Taylor, T. I. Total cross section of $\mathrm{O}_{2}$ for cold neutrons (A)

Bull. Am. Phys. Soc. 29, 71 (1954)

Fireman, E. L.

Measurement of the $\left(n, \mathrm{H}^{3}\right)$ cross section in nitrogen and its relationship to the tritium production in the atmosphere Phys. Rev. 91, 922-6 (1953)

Fireman, E. L. and Schwarzer, D.

Measurement of the tritium concentration in natural waters by a diffusion cloud chamber

Phys. Rev. 94, 385-88 (1954)

Fleeman, $J$.

The $\beta-\alpha$ transformation in tin (A)

Bull. Am. Phys. Soc. 29, 24 (1954)

Fleeman, J. and Dienes, G. J.

Mechanical properties of metals

Chapter for book Treatise on Rheology

Fowler, W. B., Johnson, A. L., Shutt, R. P., Thorndike, A. M. and Whittemore, W. L.

Diffusion cloud chambers for Cosmotron experiments Rev. Sci. Instr. (In press)

Fowler, W. B., Shutt, R. P., Thorndike, A. M. and Whittemore, W. L.

Examples of multiple pion production in $\mathrm{n}-\mathrm{p}$ collisions observed at the Cosmotron (L)

Phys. Rev. 91, 758 (1953)

Fowler, W. B., Shutt, R. P., Thorndike, A. M. and Whittemore, W. L.

Meson production in n-p collisions at Cosmotron energies Phys. Rev. 95, 1026-44 (1954)

Fowler, W. B., Lea, R. M., Shepard, W. D., Shutt, R. P.,

Thorndike, A. M. and Whittemore, W. L.

$\pi^{+}$-p scattering near $260 \mathrm{Mev}(\mathrm{L})$

Phys. Rev. 92, 832 (1953)

Fowler, W. B., Shutt, R. P., Thorndike, A. M. and Whittemore, W. L.

Production of heavy unstable particles by negative pions Phys. Rev. 93, 861-7 (1954)

Fowler, W. B., Shutt, R. P., Thorndike, A. M., and Whittemore, W. L.

Production of $V_{1}{ }^{0}$ particles by negative pions in hydrogen $(\mathrm{L})$ Phys. Rev. 91, 1287 (1953)

Frazer, B. C. (See Pepinsky, R.)

Frazer, B. C., McKeown, M. and Pepinsky, R.

Neutron diffraction studies of rochelle salt single crystals (A) Bull. Am. Phys. Soc. 29, 37 (1954)

Frazer, B. C., Pepinsky, R. and Sundara Rao, R.V.D.

Structure of ferroelectric rochelle salt (A)

Am. Crystallographic Assoc. Harvard Meeting, April, 1954
Frazer, B. C

Structure of the hydrogen-bonded ferroelectrics (A)

Am. Chem. Soc. Meeting, New York, September, 1954

Garth, R. C. (See Hughes, D. J.)

Gilbert, D. A. (See Cohen, V. W.)

Goldhaber, G. S. (See Mihelich, J. W.)

Goldhaber, G. S. and McKeown, $M$.

Disintegration scheme of $\mathrm{Br}^{80}(18 \mathrm{~min})$

Phys. Rev. 92, 356-8 (1953)

Goldhaber, G. S. and McKeown, M.

A new first excited state of an even-even nucleus: $\mathrm{Kr}^{80}(\mathrm{~A})$

Bull. Am. Phys. Soc. 28, 22 (1953)

Goldhaber, G. S. and McKeown, $M$.

New isomers in $\mathrm{Br}$ and $\mathrm{Cl}(\mathrm{A})$

Bull. Am. Phys. Soc. 29, 25 (1954)

Goldhaber, $M$.

Gamma transitions and the shell model (A)

Glasgow Conf., July, 1954

Goldhaber, $M$.

A hypothesis concerning the relations among the "new unstable particles"

Phys. Rev. 92, 1279-81 (1953)

Goldhaber, M. (See Church, E. L.)

Goldhaber, M. (See de Shalit, A.)

Hamilton, D. R. (See Weneser, J.)

Harvey, J. A. (See Carter, R. S.)

Harvey, J. A. (See Hughes, D. J.)

Harvey, J. A., Porter, C. E. and Hughes, D. J.

Variation of the $\Gamma_{\mathrm{n}}^{\circ} / \mathrm{D}$ ratio with atomic weight $(A)$

Bull. Am. Phys. Soc. 29, 57 (1954)

Hill, R. D., Salant, E. O. and Widgoff, M.

Unstable heavy particles from the Cosmotron (A)

Bull. Am. Phys. Soc. 29, 60 (1954)

Hornbostel, J. and Salant, E. O.

Artificially produced negative heavy meson (L)

Phys. Rev. 93, 902-3 (1954)

Hornyak, W. F. and Coor, T.

Capture gamma rays from the proton bombardment of $\mathrm{Be}^{9}$ Phys. Rev. 92, 675-80 (1953)

Hornyak, W. F. (See Snow, G. A.)

Hornyak, W. F. (See Hill, D. A.-Cosmotron)

Hughes, D. J. (See Kato, W. Y.)

Hughes, D. J., Kato, W. Y. and Levin, J. S.

Energy levels in hafnium isotopes (A)

Bull. Am. Phys. Soc. 28, 22 (1953)

Hughes, D. J. (See Seidl, F. G. P.)

Hughes, D. J., Garth, R. C. and Levin, J. S.

Fast neutron cross sections and nuclear level density Phys. Rev. 91, 1423-9 (1953)

Hughes, D. J. (See Levin, J. S.)

Hughes, D. J. and Palevsky, $\mathrm{H}$.

Formation of the long-lived $\mathrm{Bi}^{210}$ isomer Phys. Rev. 92, 1206-10 (1953)

Hughes, D. J. (See Palevsky, H.)

Hughes, D. J. and Harvey, J. A.

Radiation widths of nuclear energy levels

Nature 173, 942 (1954)

Hughes, D. J. (See Carter, R. S.)

Hughes, D. J. (See Harvey, J. A.)

Huntington, H. B.

Creation of displacements in radiation damage (L)

Phys. Rev. 93, 1414 (1954)

Johnson, A. L. (See Fowler, W. B.)

Kato, W. Y., Hughes, D. J. and Levin, J. S.

Determination of scattering resonance parameters from the total cross section (A)

Bull. Am. Phys. Soc. 28, 27 (1953)

Kato, W. Y. (See Hughes, D. J.) 
Kato, W. Y. (See Seidl, F. G. P.)

Keating, D. T.

Diffraction studies of possible ordering in $\alpha$-brass (L) Acta Metallurgica

Kikuchi, C. and Cohen, V. W.

Paramagnetic resonance absorption of carbazyl and hydrazyl. Phys. Rev. 93, 394-9 (1954)

Kikuchi, C., Sirvetz, M. H. and Cohen, V. W. Paramagnetic resonance hyperfine structure of $\mathrm{V}^{50}$ and $\mathrm{V}^{51}$ Phys. Rev. 92, 109-13 (1953)

Koski, W. (See Cohen, V. W.)

Kraushaar, J. J. and Pound, R. V.

The directional correlation and electric quadrupole interaction in the $\gamma-\gamma$ cascade of $48.7-\mathrm{min} \mathrm{Cd}^{111 \mathrm{~m}}(\mathrm{~L})$ Phys. Rev. 92, 522 (1953)

Kraushaar, J. J., Mihelich, J. W. and Sunyar, A. W. The directional correlation of the $\gamma$-rays of $A^{38}$ Phys. Rev. 95, 456 (1954)

Kraushaar, J. J. Gamma-gamma directional correlation experiments with $\mathrm{Mo}^{93 \mathrm{~m}}$

Phys. Rev. 92, 318-20 (1953)

Kroll, N. M. and Ruderman, M. A.

A theorem on photo-meson production near threshold and the suppression of pairs in pseudoscalar meson theory Phys. Rev. 93, 233 (1954)

Levin, J. S. (See Kato, W. Y.)

Levin, J. S. (See Hughes, D. J.)

Levin, J. S. (See Seidl, F. G. P.)

Levin, J. S. and Hughes, D. J.

Flux depression and self-protection effects in production of radiocobalt

Nucleonics 11, 8 (1953)

Levin, J. S. and Hughes, D. J.

Measurements of neutron resonance parameters in $\mathrm{U}, \mathrm{Th}$, $\mathrm{Hg}$, and $\mathrm{Au}(\mathrm{A})$

Bull. Am. Phys. Soc. 29, 57 (1954)

Levy, P. W. and Dienes, G. J.

Color centers produced by reactor neutron irradiation (A)

Bristol Conf. on Defects in Crystalline Solids, Bristol, England, July, 1954

Levy, P. W. and Dienes, G. J. Irradiation-induced optical absorption in $\mathrm{Al}_{2} \mathrm{O}_{3}$ (A) Bull. Am. Phys. Soc. 29, 11 (1954)

Lindenbaum, S. J., Pevsner, A., Rainwater, J. and Williams, R. Elastic scattering of $80 \mathrm{Mev} \pi$-mesons on aluminum (A) Bull. Am. Phys. Soc. 29, 28 (1954)

Lindenbaum, S. J. (See Yuan, L. C. L.Cosmotron)

Lindenbaum, S. J. and Yuan, L. C. L.

The interaction cross-section of hydrogen and heavier elements for $450-\mathrm{Mev}$ negative pions and $340-\mathrm{Mev}$ positive pions (L)

Phys. Rev. 92, 1578 (1953)

Lindenbaum, S. J. and Yuan, L. C. L.

The production of positive and negative pions in beryllium by 1.0 - and 2.3 - Bev protons (A)

Bull. Am. Phys. Soc. 29, 50 (1954)

Lindenbaum, S. J. and Yuan, L. C. L.

The total interaction cross section of hydrogen for positive and negative $200-700 \mathrm{Mev}$ pions (A)

Bull. Am. Phys. Soc. 28, 13 (1953)

Madansky, L. F. (See Cool, R. L.)

Mckeown, M. (See Mihelich, J. W.)

McKeown, M. and Goldhaber, G. S. Distintegration scheme of $\mathrm{Br}^{\mathrm{*} 0}$ (18 min)

Phys. Rev. 92, 356-8 (1953)

McKeown, M. (Sce Muehlhause, C. O.)

McKeown, M. (See Pepinsky, R.)
McKeown, M. (See Frazer, B. C.)

McKeown, M. (See Reynolds, A. W.)

McKeown, M. (See Goldhaber, G. S.)

McKeown, M. and Katcoff, $S$. Radiations of $53-\mathrm{min} \mathrm{I}^{134}$ and $86-\mathrm{sec} \mathrm{I}^{136}$

Phys. Rev. 94, 965.7 (1954)

McReynolds, A. W.

Inelastic scattering of thermal neutrons in lead Phys. Rev. 91, 1368-71 (1953)

McReynolds, A. W. and Riste, T. Magnetic neutron diffraction from $\mathrm{Fe}_{3} \mathrm{O}_{4}$ Phys. Rev. 95, 1161-61 (1954)

McReynolds, A. W., Augustyniak, W., McKeown, M. and

Rosenblatt, D. B.

Neutron irradiation effects at low temperature $-\mathrm{Cu}$ and Al (A)

Bull. Am. Phys. Soc. 29, 19 (1954)

Mihelich, J. W., Goldhaber, G. S. and McKeown, M. Decay schemes of the 5.5 -hr isomer of $\mathrm{Hf}^{180 \mathrm{n}}$ (A) Bull. Am. Phys. Soc. 29, 57 (1954)

Mihelich, J. W. (See Kraushaar, J. J.)

Mihelich, J. W. The disintegration of $\mathrm{Ta}^{182}(\mathrm{~A})$ Bull. Am. Phys. Soc. 29, 38 (1954)

Mihelich, J. W., Schardt, A. W. and Segré, E. Energy levels in $\mathrm{Po}^{210}$ Phys. Rev. (In press)

Mihelich, J. W., Gillon, L. P., Gopalakrishnan, K. and de Shalit, A.

Nuclear spectroscopy of neutron-deficient $\mathrm{Hg}$ isotopes Phys. Rev. 93, 124-35 (1954)

Mihelich, J. W. and de Shalit, A. Some regularities in the nuclear level spacings of $\mathrm{Hg}, \mathrm{Au}$, and $\mathrm{Pt}$

Phys, Rev. 93, 135-9 (1954)

Motz, H. T.

Slow neutron capture gamma rays from titanium (A) Bull. Am. Phys. Soc. 28, 21 (1954)

Muehlhause, C. O., der Mateosian, E. and McKeown, M. Effects of preparation and temperature on the response of $\mathrm{NaI}$ phosphorus (A)

Bull. Am. Phys. Soc. 29, 10 (1954)

Muehlhause, C. O.

Some neutron detection investigations BNL 242 (T-38)

Muether, H. R. (See Palevsky, H.)

Myers, V. W. (See Carter, R. S.)

Oliver, A. R. (See Blau, M.)

Palevsky, H. (See Seidl, F. G. P.)

Palevsky, H. (See Hughes, D. J.)

Palevsky, $H$. and Hughes, D. J. Magnetic inelastic scattering of slow neutrons (L) Phys. Rev. 92, 202-3 (1953)

Palevsky, M. and Eisberg, R. M. Magnetic scattering of cold neutrons by gaseous oxygen (A) Bull. Am. Phys. Soc. 29, 71 (1954)

Palevsky, H., Carter, R. S., Eisberg, R. M. and Hughes, D. J. The neutron absorption cross section of $\mathrm{U}^{235}$ at $2200 \mathrm{~m} / \mathrm{sec}$ Phys. Rev. 94, 1088-9 (1954)

Palevsky, H., Muether, H. R. and Stolovy, A. Scintillation detector for the Brookhaven fast chopper (A) Bull. Am. Phys. Soc. 28, 16 (1953)

Palevsky, H. (See Carter, R. S.)

Palevsky, H. (See Eisberg, R. M.)

Pepinsky, R., Frazer, B. C., McKeown, M. and Oleson, N. Miniaturized neutron diffraction goniometers (A) Am. Crystallographic Assoc., Harvard Meeting, April 1954 
Pepinsky, R. (See Frazer, B. C.)

Pepinsky, R., Frazer, B. C. and McKeown, M.

New goniometers for single-crystal neutron diffraction studies

Rev. Sci. Instr. 25, 699-703 (1954)

Piccioni, O. (See Cool, R. L.)

Pilcher, V. E., Carter, R. S. and Stolovy, A.

Neutron resonances in holmium, thulium, and lutecium (A)

Bull. Am. Phys. Soc. 29, 57 (1954)

Porter, C. E. Jr.

Neutron cross sections of light nuclei (A)

Bull. Am. Phys. Soc. 29, 25 (1954)

Porter, C. E., Jr. (See Harvey, J. A.)

Pound, R. V. (See Kraushaar, J. J.)

Pryce, M. H. L. (See Alburger, D. E.)

Ramsey, N. F. (See Cohen, V. W.)

Reilly, E. C.

A combination high vacuum and pressure valve (N)

Rev. Sci. Instr. 24, 875-6 (1953)

Reilly, E. G.

An insulated union for metal tubing (N)

Rev. Sci. Instr. 24, 879 (1953)

Rickey, M. W. (See Eisberg, R. M.)

Riste, T. (See McReynolds, A. W.)

Rosenblatt, D. B.

Effects of a primeval endowment of $U^{236}$

Phys. Rev. 91, 1474-5 (1953)

Rosenblatt, D. B. (See McReynolds, A. W.)

Rosenblatt, D. B., Smoluchowski, R. and Dienes, G. J.

Radiation-induced changes in the electrical resistivity of $\alpha$ brass (A)

Bull. Am. Phys. Soc. 29, 19 (1954)

Ruderman, M. A. (See Kroll, N. M.)

Salant, E. O. (See Hornbostel, J.)

Salant, E. O. (See Hill, R. D.)

Schwarzer, D. (See Fireman, E. L.)

Segré, E. (See Mihelich, J. W.)

Seidl, F. G. P., Hughes, D. J., Palevsky, H., Levin, J. S., Kato,

W. Y. and Sjostrand, N. G.

"Fast chopper" time-of-flight measurement of neutron resonances

Phys. Rev. 95, 476 (1954)

Seidl, F. G. P.

Recent measurements on the lowest neutron scattering resonance in cobalt (A)

Bull. Am. Phys. Soc. 28, 27 (1953)

Shutt, R. P. (See Fowler, W. B.)

Shutt, R. P.

Experimental results with the Brookhaven Cosmotron (A) Natl. Acad. Sci. Meeting, November, 1953
Sirvetz, M. H. (See Kikuchı, C.)

Sjostrand, N. G. (See Seidl, F. G. P.)

Smith, J. E. (See Caulton, M.)

Smith, J. E. (See Blau, M.)

Snow, G. A. (See Hill, D. A.)

Snow, G. A., Coor, T., Hill, D. A., Hornyak, W. F. and Smith, L. W.

Experiments with neutrons from the Cosmotron. II (A) Bull. Am. Phys. Soc. 29, 54 (1954)

Snow, G. A., Sternheimer, R. M. and Yang, C. M.

Polarization of nucleons elastically scattered from nuclei(L)

Phys. Rev. 94, 1073-4 (1954)

Snyder, H. S. (See Blewett, M. H.-Cosmotron)

Stolovy, A. (See Pilcher, V. E.)

Stolovy, A. (See Palevsky, H.)

Sunyar, A. W.

The decay of $\mathrm{Ho}^{166}$

Phys. Rev. 93, 1345 (1954)

Sunyar, A. W. (See Kraushaar, J. J.)

Sunyar, A. W.

Lifetime measurements of fast E2 transitions in even-even nuclei $(A)$

Bull. Am. Phys. Soc. 29, 38 (1954)

Sunyar, A. W.

Short lived metastable states of $\mathrm{W}^{182}$

Phys. Rev. 93, 1122-3 (1954)

Thorndike, A. M. Cosmotron production of heavy mesons (A)

Glasgow Conf., July, 1954

Thorndike, A. M.

Cosmotron results on pion-nucleon interactions (A)

Thorndike, A. M. (See Fowler, W. B.)

Townes, H. C., Cohen, V. W., Silvey, G., Burke, B. F., Strand-

berg, M., Hardy, W. A. and Parker, G. W.

The nuclear moments of $\mathrm{Se}^{79}$

Phys. Rev. 92, 1532-7 (1953)

Vineyard, G. H. and Dienes, G. J.

On the theory of defect concentration in crystals

Phys. Rev. 93, 265-8 (1954)

Weneser, J.

Inner bremstrahlung and the magnetic moment of the neutrino ( $L$ )

Phys. Rev. 91, 1025 (1953)

Weneser, J. and Hamilton, D. R.

A theorem concerning angular correlation

Phys. Rev. 92, 321-2 (1953)

Whittemore, W. L. (See Fowler, W. B.)

Widgoff, M. (See Hill, R. D.)

Wu, C. S. (See der Mateosian, E.) 


\title{
B. OFFICERS AND SCIENTIFIC STAFF
}

\author{
Leland J. Haworth, Director \\ Gerald F. Tape, Deputy Director
}

Robert A. Patterson, Assistant Director

William H. Fields, Assistant Director

Leland J. Haworth, Chairman, Accelerator Development Department

Howard J. Curtis, Chairman, Biology Department

Richard W. Dodson, Chairman, Chemistry Department

George B. Collins, Chairman, Cosmotron Department

J.B.H. Kuper, Chairman, Instrumentation and Health Physics Department

Lee E. Farr, Chairman, Medical Department

Clarke Williams, Chairman, Nuclear Engineering Department

Samuel A. Goudsmit, Chairman, Physics Department

Marvin Fox, Chairman, Reactor Department

J. Georges Peter, Manager, Architectural Planning \& Plant Maintenance Department

H. Russell Cort, Budget Officer

Lewis R. Burchill, Controller

Charles F. Dunbar, Legal Counsel

Laurence R. Swart, Personnel Officer

Frederick H. Williams, Security Officer

\section{Accelerator Development Department}

Leland J. Haworth, Chairman

Roger R. Adams

Richard A. Beth

(on leave from Western Reserve Univ.)

John W. Bittner

John P. Blewett

M. Hildred Blewett

Nicholas C. Christofilos

James $G$. Cottingham

Charles E. Falk

George K. Green

Charles L. Gould

Donald W. Huszagh

David D. Jacobus

David A. Kassner

Ralph R. Kassner

John D. Kiesling

Calman Lasky

John J. Mede*

Walter W. Merkle

James P. Palmer

Robert H. Phillips

Martin Plotkin

Irving J. Polk

Eugene C. Raka

Raymond $\mathrm{H}$. Rheaume

Albert P. Schlafke, Jr.

* Terminated before June 30, 1954.
Edward E. Shelton

Maxwell M. Small

Julius Spiro

Lincoln D. Stoughton

Arthur M. Vash

Biology Department

Howard J. Curtis, Chairman

Irwin Berman

(graduate student from NYU)

Charles C. Bowen

(on US Public Health Service Fellowship)

Vaughan T. Bowen

Richard S. Caldecott

Arnold M. Clark

(on leave from Univ. of Delaware)

Phelps P. Crump

Elwood Dollinger, Jr.

(on US Public Health Service Fellowship)

Abraham Edelmann

Donald J. Fluke

Frederick Forro, Jr.

Martin Gibbs

Monroe S. Glitzer

(on US Public Health Service Fellowship)

Gertrude A. Heidenthal

(on leave from Russell Sage College)

James R. Klein

Robert C. King

Daniel E. Koshland, Jr.

Marian E. Koshland 
Calvin F. Konzak

Arthur G. Kowalsky (Postdoctoral appointment)

Montrose J. Moses

Leslie F. Nims

Lynn M. Paege

(assigned from Frankford Arsenal)

Sherwood M. Reichard

(graduate student from NYU)

Eugene M. Renkin

Leon Z. Saunders

Seymour Shapiro

Willard R. Singleton

Arnold H. Sparrow

Robert Steele

Dale $M$. Steffensen

Elizabeth Sternheimer*

(on US Public Health Service Fellowship)

Tom T. Stonier (graduate student from Yale)

Raymond R. Walsh

Dow Owen Woodward

(on US Public Health Service Fellowship)

Chemistry Department

Richard W. Dodson, Chairman

Augustine $\mathrm{O}$. Allen

R. Christian Anderson

Elizabeth W. Baker

Nathaniel F. Barr

(Postdoctoral appointment)

Jacob Bigeleisen

Albert A. Caretto, Jr.

(Postdoctoral appointment)

Lee G. Carpenter

(graduate student from Columbia)

David R. Christman

Lester M. Corliss

James B. Cumming

(Postdoctoral appointment)

Raymond Davis, Jr.

Nancy E. Day

Robert Duffield*

(on leave from Univ. of Illinois)

Norman Elliott

Simon Freed

Gerhart Friedlander

Lewis Friedman

Floyd Gould

(graduate student from Columbia)

Patricia R. Hansell

Garman Harbottle

Julius $M$. Hastings

Virginia D. Hogan*

Ralph A. Horne

(graduate student from Columbia)

Jerome Hudis

Adolph P. Irsa

Seymour Katcoff

Charles H. Leigh

(on leave from Syracuse)

Sheldon L. Matlow

(Postdoctoral appointment)

Donald G. Miller

(Postdoctoral appointment)

* Terminated before June 30, 1954.
John A. Miskel

Elinor F. Norton

Henry R. Owen

Eduardo Penna Franca

(on leave from Oswaldo Cruz Institute)

Morris L. Perlman

Peter Riesz

(Postdoctoral appointment)

Walter G. Rothschild

J. Keith Rowley

William Rubinson

Jerome Saldick

Kenneth Sancier*

Edward V. Sayre

Oliver A. Schaeffer

Robert H. Schuler

Harold A. Schwarz

Morris Slavin

Raymond W. Stoenner

Nathan Sugarman*

(on leave from Univ. of Chicago)

Sydney $\mathrm{O}$. Thompson

Anthony Turkevich*

(on leave from Univ. of Chicago)

Joan P. Welker

Ralph E. Weston, Jr.

Alfred P. Wolf

Richard L. Wolfgang

Max Wolfsberg

Arvid V. Zuber*

(graduate student from Columbia)

\section{Cosmotron Department}

George B. Collins, Chairman

Robert K. Adair

Henry A. Blumenfeld

(graduate student from Columbia)

Francis F. Chen

(graduate student from Harvard)

Richard Christian*

(on leave from Los Alamos Scientific Lab.)

Ernest D. Courant

Edward W. Dexter

Salvatore T. Giordano

Christopher P. Leavitt

(Postdoctoral appointment)

Isador J. Livant

Leon Madansky*

(on leave from Johns Hopkins Univ.)

Richard Madey

Robert L. Mills

(Postdoctoral appointment)

William $\mathrm{H}$. Moore

Thomas W. Morris

(graduate student from Yale)

Richard S. Preston

(graduate student from Yale)

Burton Richter*

(graduate student from Yale)

Stuart L. Ridgway

(Postdoctoral appointment)

Edwin J. Rogers

Marc Ross

(Postdoctoral appointment)

Anatole M. Shapiro

* Terminated before June 30, 1954. 
Lyle W. Smith

Rudolph Sternheimer

Clifford E. Swartz

Clarence M. Turner

Robert M. Walker (graduate student from Yale)

Daniel Willard (Postdoctoral appointment)

Chen Ning Yang* (on leave from Institute for Advanced Studies) Luke C. Yuan

\section{Director's Office}

P. Gerald Kruger*

(on leave from Univ. of Illinois)

Stuart Rideout

Instrumentation and Health Physics

J. B. H. Kuper, Chairman

Donald S. Beard

Maurice R. Beebe

William Bernstein

Richard H. Boutelle

William F. Burditt*

Robert L. Chase

Frederick P. Cowan

Edward H. Foster

Lee Gemmell

Martin H. Graham

John S. Handloser

William A. Higinbotham

Byron E. Keene

Lionel Lewis

Julia Marshall*

Casimir Z. Nawrocki

Lloyd A. Nevala

Joseph F. O'Brien

Frederick B. Oleson

Leigh F. Phillips

Seymour Rankowitz

Richard C. Roth

Alois W. Schardt*

William D. Small*

Arthur J. Solari

Charles F. Stearns

Jerome Weiss

Max M. Weiss

\section{Medical Department}

Lee E. Farr, Chairman

Paul E. Carson, Jr.

Hadley L. Conn, Jr.

George C. Cotzias

Lewis K. Dahl

Frank Dresdale*

Ruth M. Drew

Edward T. Dunham

Otho D. Easterday

Lewis I. Gidez

John T. Godwin

Lawrence V. Hankes

David L. Jellinger

Charles G. Lewallen

Robert A. Love

Laurence S. Maynard

* Terminated before June 30, 1954.
Edwin A. Popenoe

James Robertson

(on military leave)

Irving Serlin

Claire J. Shellabarger

F. Marrott Sinex

Bernard G. Stall

Elmer E. Stickley

Richard D. Stoner

Robert Stroud*

Daniel Tosteson* (on military leave)

Donald D. Van Slyke

William Wolins

Nuclear Engineering Department

Clarke Williams, Chairman

James E. Atherton, Jr.

David S. Ballantine

Hampden O. Banks, Jr.

Richard G. Bauman

(assigned from B.F. Goodrich Co.)

Joseph S. Bryner

Frank V. Caccavo

Jack Chernick

Noel R. Corngold

Robert F. Doering

Kenneth W. Downes

Orrington E. Dwyer

James J. Egan

Harold L. Falkenberry (assigned from Tennessee Valley Authority)

Harmon L. Finston

Joseph A. Fleck, Ir.

Aaron J. Friedland*

William S. Ginell

Althea Glines

Eli I. Goodman

David H. Gurinsky

Loranus P. Hatch

Leonard R. Heintze

Raymond J. Heus

Fred Hittman

Robert J. Hoe*

(graduate student from Cornell)

Frederick L. Horn

Robert J. Isler

Otto F. Kammerer

Irving Kaplan

Carl J. Klamut

Ernest A. Knipp, Jr. (graduate student from Yale)

Herbert J. C. Kouts

Theodore M. Littman

Bernard Manowitz

Francis T. Miles

Bernard Mozer

Sophie Oleksa

Robert D. Pierce

(graduate student from Univ. of Michigan)

Glenn A. Price

Chad J. Raseman

William H. Regan, Jr.

Leslie G. Ritner

(deceased)

* Terminated before June 30, 1954. 
William A, Robba

Jerome Sadofsky

Thomas V. Sheehan

(on leave from Standard Oil Co. of Indiana)

Rudolph Sher

Melvin Silberberg

Louis G. Stang, Jr.

Harry $H$. Steinhauser, Jr.

(on leave from RPI)

Gerald Strickland

Herbert Susskind

Robert J. Teitel

Walter D. Tucker

Charles $\mathrm{H}$. Waide

John R. Weeks

Joel Weisman

Edward Wirsing, Jr.

Richard H. Wiswall, Jr.

\section{Physics Department}

Samuel A. Goudsmit, Chairman

David E. Alburger

John J. Antal

(assigned from Watertown Arsenal)

Charles P. Baker

Marietta E. Blau

Stewart D. Bloom

Ernest M. Bolze

Robert S. Carter

Martin Caulton

(graduate student from RPI)

David D. Clark

(Postdoctoral appointment)

Victor W'. Cohen

Rodney L. Cool

Thomas Coor, Jr.*

Edward der Mateosian

George J. Dienes

James E. Draper

Robert M. Eisberg

(Postdoctoral appointment)

Edward L. Fireman

Jerome Fleeman

William B. Fowler

Benjamin C. Frazer

(on leave from Penn. State College)

Donald A. Gilbert

G. Norris Glasoe

Murrey D. Goldberg*

Gertrude S. Goldhaber

Maurice Goldhaber

Donald R. Hamilton*

(on leave from Princeton)

William M. Harris

John A. Harvey

Edward L. Healey

David A. Hill*

(graduate student from MIT)

Robert D. Hill*

(on leave from Univ. of Illinois)

John Hornbostel

William F. Hornyak

(on leave of absence)

Donald Hughes

(on leave of absence)

* Terminated before June 30, 1954.
Thomas $\mathrm{H}$. Johnson

(on leave of absence)

Walter Y Kato*

(graduate student from Penn. State College)

David T. Keating

V. Paul Kenney (graduate student from Fordham)

David Kleinman* (Postdoctoral appointment)

Walter L. Kosiba

Jack J. Kraushaar*

(Postdoctoral appointment)

Jane Levin

Jules S. Levin

(graduate student from Cornell)

Paul W. Levy

Phyllis O. Levy*

Seymour J. Lindenbaum

Andrew W. McReynolds

Robert S. Margulies

(graduate student from Cornell)

John W. Mihelich

Henry T. Motz (on leave of absence)

Vernon Myers*

(on leave from Penn. State College)

Carl O. Muehlhause

Harry Palevsky

Simon Pasternack

(assistant editor of The Physical Review)

Oreste Piccioni

Valter E. Pilcher

(graduate student from No. Carolina State College)

Charles E. Porter, Jr.

Martin E. Rickey

David B. Rosenblatt (assigned from Frankford Arsenal)

Hyman Rosenwasser (assigned from Picatinny Arsenal)

Edward O. Salant

William T. Scott* (on leave from Smith College)

Frederick G. P. Seidl

Ralph P. Shutt

Harvey P. Sleeper, Jr.

Joseph E. Smith

Lincoln G. Smith

George A. Snow

Hartland S. Snyder

Alexander Stolovy (graduate student from NYU)

Andrew W. Sunyar

Alan Thorndike

Bert J. Toppel

George H. Vineyard*

(on leave from University of Missouri)

William A. Wallenmeyer, Jr. (graduate student from Purdue Univ.)

Harvey E. Wegner

Joseph Weneser

William L. Whittemore

Mildred Widgoff*

Reactor Department

Marvin Fox, Chairman

* Terminated before June 30, 1954. 
John E. Binns

Bertram Brockhouse*

(assigned from Atomic Encrgy of Canada, Ltd)

Wallace DeLaguna (assigned from US Geological Survey)

Bruce D. Dodd* (assigned from Consolidated Vultee Aircraft)

John A. Dooley

John J. Floyd

Hugie L. Foote, Jr. (Postdoctoral appointment)

Eugene R. French* (assigned from US Geological Survey)

Walter W. Godsin (assigned from Westinghouse Corp.)

Harry H. Landon, Jr.

Walter Lones

Robert A. McCormick (assigned from US Weather Bureau)

Lowell McLean

Charles L. Osborne

* Terminated before June $30,1954$.
Jack E. Phillips

Depuyster G. Pitcher

Robert W. Powell

Edward Prince (assigned from Bell Telephone Co.)

Alonzo C. Rand, Jr.

Melvin Reier*

Brice Rustad

$$
\text { (assigned from Columbia) }
$$

Vance L. Sailor

Robert D. Schamberger

Ferdinand J. Shore, Jr.

Irving A. Singer

Maynard E. Smith

Raymond C. Wanta* (assigned from US Weather Bureau)

William $\mathrm{T}$. Warner

Alfred M. Warren

(assigned from US Geological Survey)

Richard E. Wood

(graduate student from Univ. of Utah)

* Terminated before June 30, 1954 